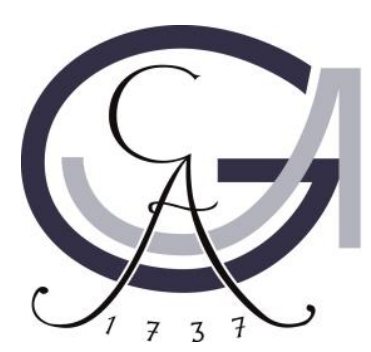

\title{
A method for the genetically encoded incorporation of FRET pairs into proteins
}

\author{
Dissertation \\ zur Erlangung des mathematisch-naturwissenschaftlichen Doktorgrades \\ "Doctor rerum naturalium" \\ der Georg-August-Universität Göttingen \\ im Promotionsprogramm Biologie \\ der Georg-August University School of Science (GAUSS)
}

vorgelegt von

\section{Christoph Lammers}

aus Würselen 


\section{$\underline{\text { Betreuungsausschuss }}$}

Jun.-Prof. Dr. Heinz Neumann, Angewandte Synthetische Biologie, Institut für Mikrobiologie und Genetik

Prof. Dr. Jörg Stülke, Allgemeine Mikrobiolgie, Institut für Mikrobiologie und Genetik

\section{Mitglieder der Prüfungskommission}

Referent: Jun.-Prof. Dr. Heinz Neumann, Angewandte Synthetische Biologie, Institut für Mikrobiologie und Genetik

Korreferent: Prof. Dr. Jörg Stülke, Allgemeine Mikrobiolgie, Institut für Mikrobiologie und Genetik

\section{Weitere Mitglieder der Prüfungskommission:}

Prof. Dr. Ralf Ficner, Molekulare Strukturbiologie, Institut für Mikrobiologie und Genetik

Prof. Dr. Kai Tittmann, Bioanalytik, Albrecht-von-Haller-Institut

Prof. Dr. Rolf Daniel, Genomische und Angewandte Mikrobiologie, Institut für Mikrobiologie und Genetik

Dr. Fabian Commichau, Allgemeine Mikrobiolgie, Institut für Mikrobiologie und Genetik 


\section{Table of Contents}

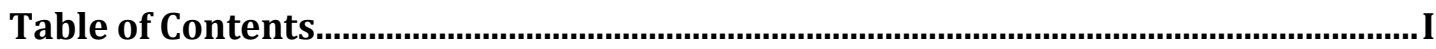

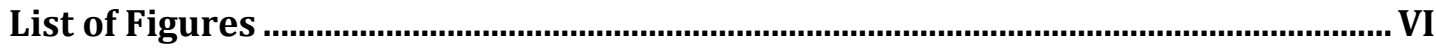

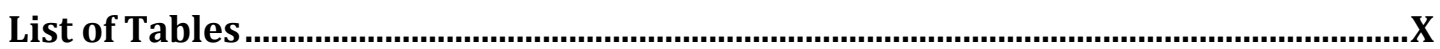

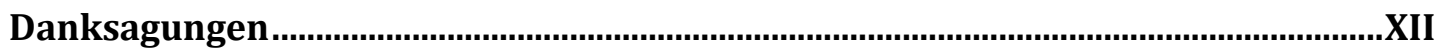

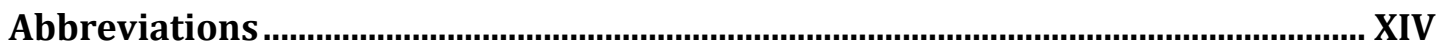

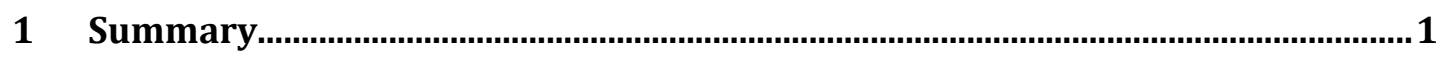

2 Introduction

$2.1 \quad$ Proteins Encoded By The Genetic Code .................................................. 3

2.2 Expansion And Modification of The Genetic Code...................................... 5

2.3 Limitations And Optimizations of The Expanded Genetic Code .................. 8

2.4 Applications of The Expanded Genetic Code .......................................... 13

2.5 The Genetically Encoded Incorporation of FRET Pairs ............................ 15

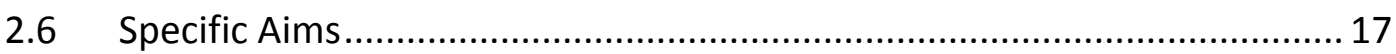

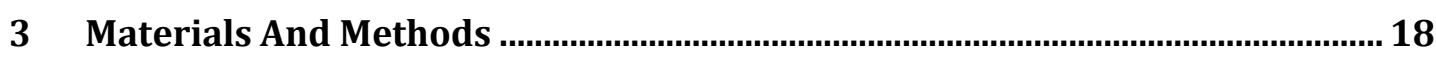

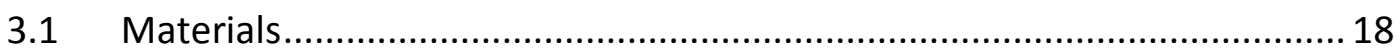

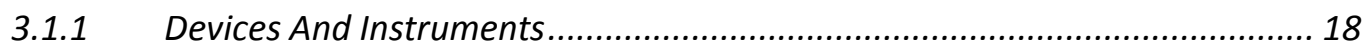

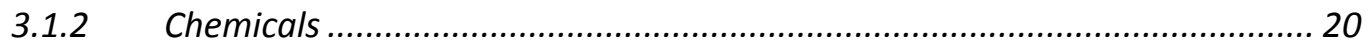

3.1.3 Consumables And Other Materials ......................................................... 20

3.1.4 DNA, Protein And RNA Size Standards .................................................. 22

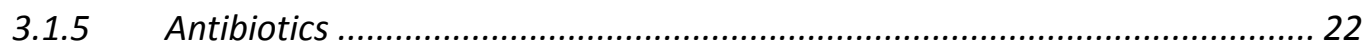

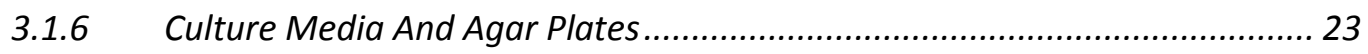

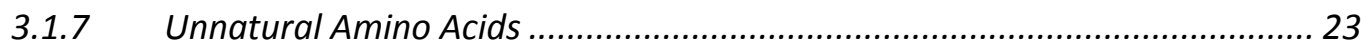

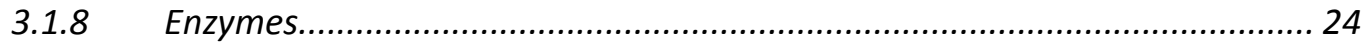

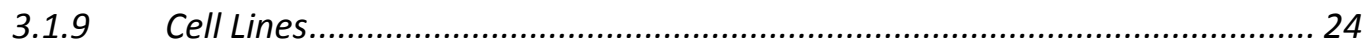

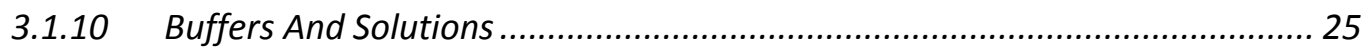




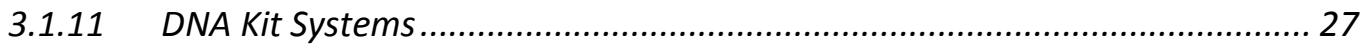

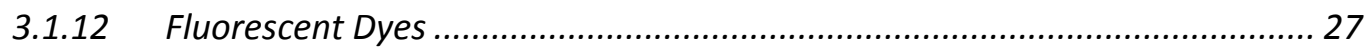

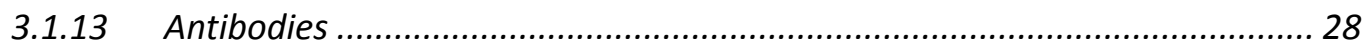

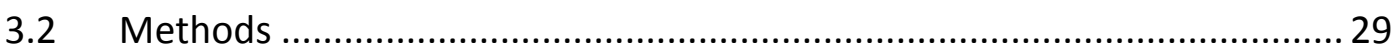

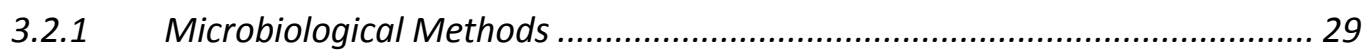

3.2.1.1 Chemical Competent Cells ............................................................... 29

3.2.1.2 Electro Competent Cells................................................................... 29

3.2.1.3 Transformation of Chemical Competent Cells ........................................ 29

3.2.1.4 Transformation of Electro Competent Cells ............................................ 30

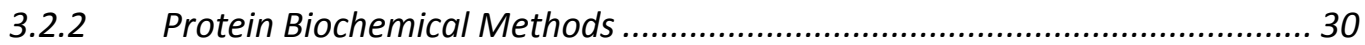

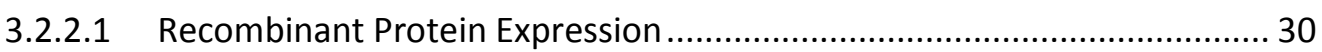

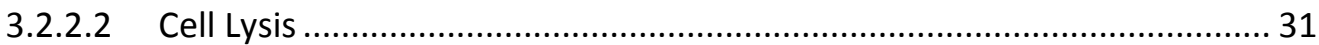

3.2.2.3 Discontinuous Sodium Dodecyl Sulfate Polyacrylamide Gel Electrophoresis 31

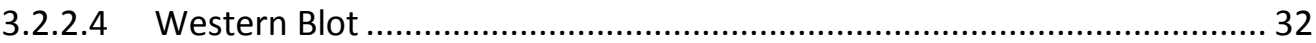

3.2.2.5 Measuring Protein Concentration ........................................................ 33

3.2.2.6 Small Scale GST-MBP Expression And Purification .................................. 33

3.2.2.7 Small Scale Histone H3 Expression And Purification................................... 34

3.2.2.8 Large Scale Nanobody NbSyn2 Expression And Purification ..................... 35

3.2.2.9 Expression And Purification of Isotopically Labeled sfGFP ....................... 35

3.2.2.10 Protein Expression For In-Cell NMR Measurements............................. 36

3.2.2.11 Labeling of Proteins With Fluorophores ............................................ 36

3.2.2.12 Fluorescence Measurement With A Plate Reader .............................. 37

3.2.2.13 Fluorescence-Activated Cell Sorting ................................................... 37

3.2.3 Nucleic Acid Biochemical Methods .......................................................... 38

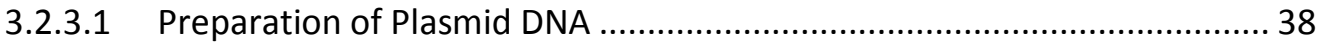

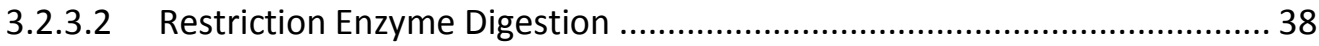

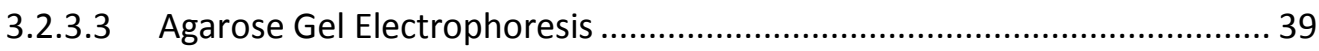

3.2.3.4 Extraction of DNA From Agarose Gels .................................................... 40 


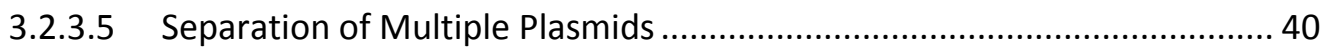

3.2.3.6 Ligation of Two Double-Stranded DNA Fragments .................................. 41

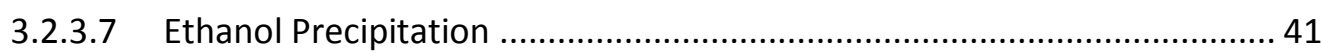

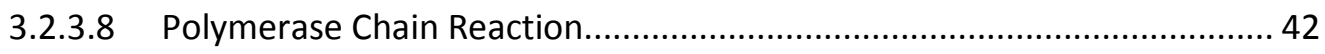

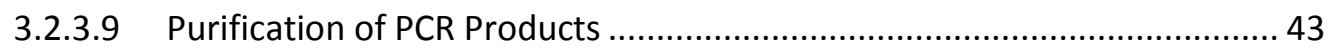

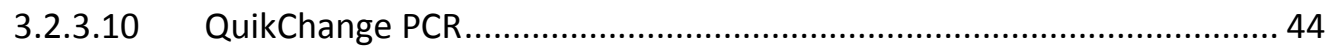

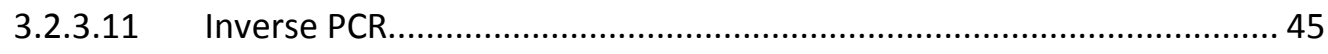

3.2.3.12 Measuring Nucleic Acid Concentration.............................................. 47

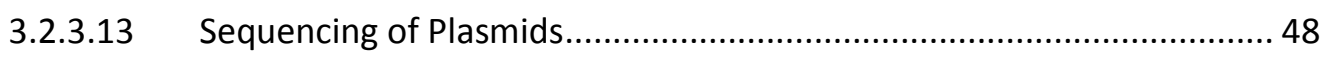

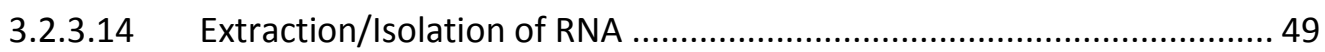

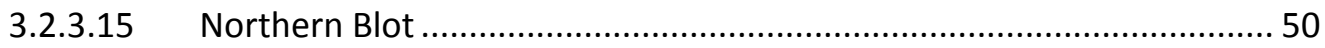

3.2.3.16 Transcription With T7 RNA Polymerase .............................................. 51

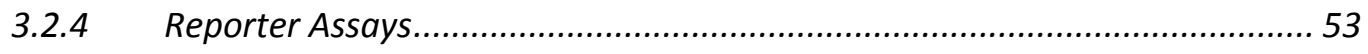

3.2.4.1 Chloramphenicol Reporter Assay......................................................... 53

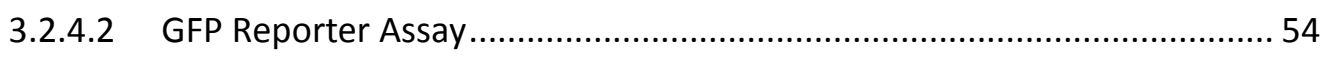

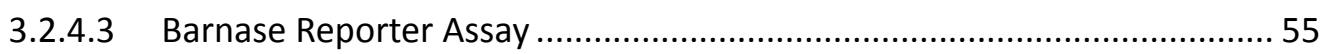

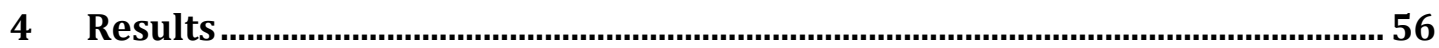

4.1 Abundance And Activity of The aaRS/tRNA Pairs PyIS/PyIT And MjYRS/MjYT 56

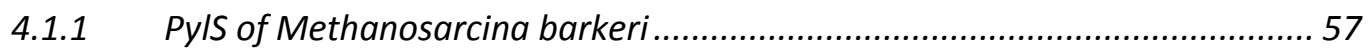

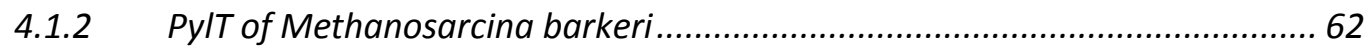

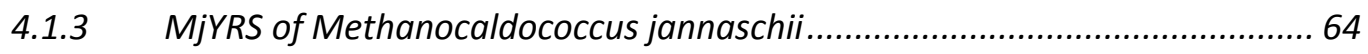

4.1.4 MjYT of Methanocaldococcus jannaschii .................................................. 67

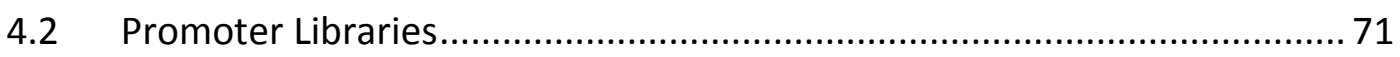

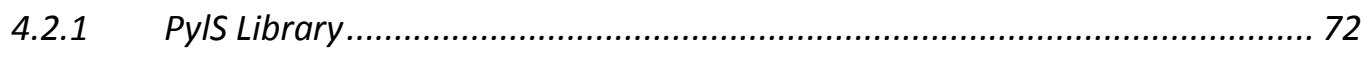

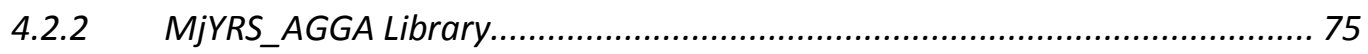

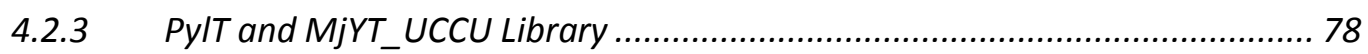

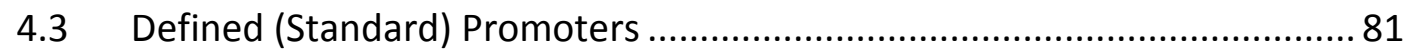




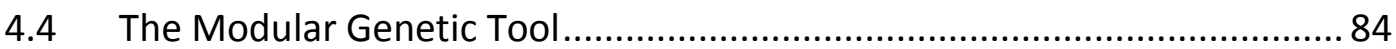

4.4.1 The Concept of The Modular Genetic Tool................................................ 84

4.4.2 The Construction of The Modular Genetic Tool .......................................... 85

4.4.3 Monitoring The Power of The Modular Genetic Tool.................................. 90

4.4.4 The Combination of The Modular Genetic Tool and The Promoter Libraries. 92

4.4.5 Alternative Strategies For Screening The Modular Genetic Library Tool ....... 96

4.4.6 The Combination of The Modular Genetic Tool And Defined (Standard) Promoters. 100

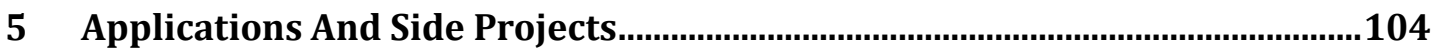

5.1 Evolution of A Quadruplet Decoding MjAzFRS ….................................. 104

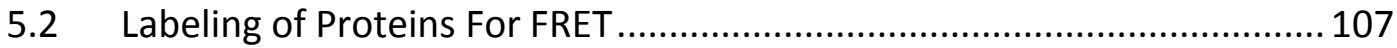

5.3 Fluorescently Labeled Nanobodies .................................................... 111

5.4 Orthogonal Ribosome Assisted In-Cell NMR Spectroscopy ...................... 114

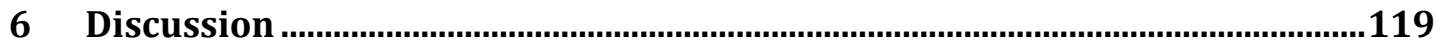

6.1 Balancing An Expanded Genetic Code System ...................................... 119

6.2 Reducing The Metabolic Burden ..................................................... 122

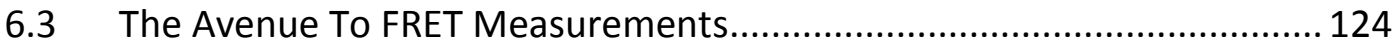

6.4 Applications And Side Projects: Nanobodies And In-Cell NMR ................ 125

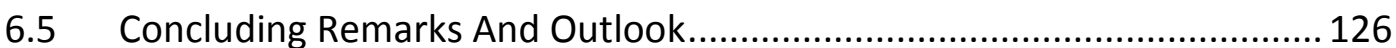

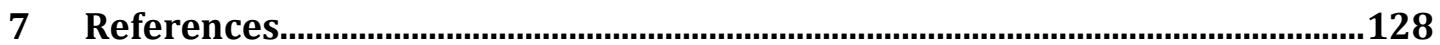

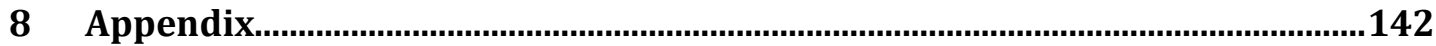

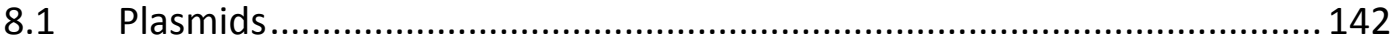

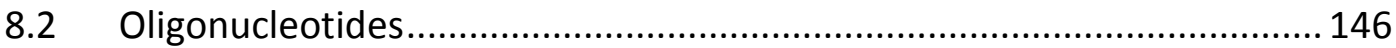

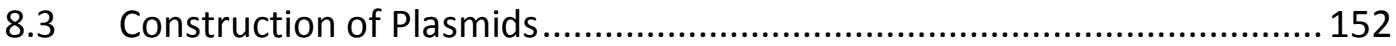

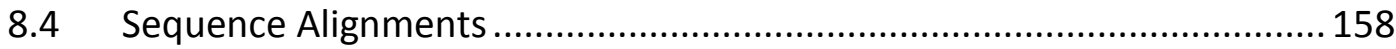

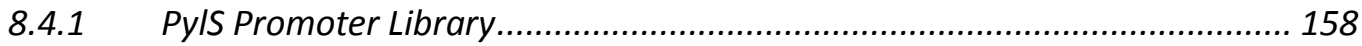

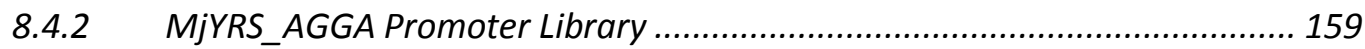

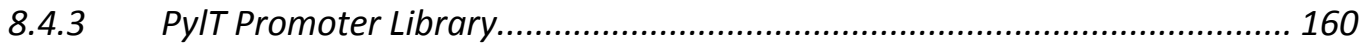


8.4.4 MjYT_UCCU Promoter Library............................................................... 161

8.5 In-Cell sfGFP Fluorescence Measurements .......................................... 162

8.5.1 Initial Conditions For In-Cell NMR Measurements .................................... 162

8.5.2 Optimization For In-Cell NMR Measurements .......................................... 163

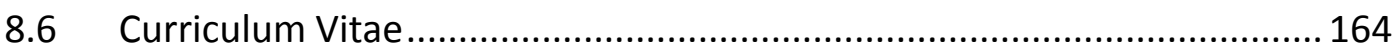




\section{List of Figures}

Figure 2.1: Schematic view for genetic code expansion.

Figure 2.2: General selection scheme for the evolution of aminoacyl-tRNA synthetase (aaRS) substrate specificities for unnatural amino acids (UAAs) ${ }^{[44]}$..... 7

Figure 3.1: Schematic view of QuikChange cycles

Figure 3.2: Schematic representation of the restriction enzyme-mediated inverse $\mathrm{PCR}^{[135]} .46$

Figure 3.3: Schematic view of the chloramphenicol reporter assay..... 53

Figure 3.4: Schematic view of the GFP reporter assay. 54

Figure 3.5: Schematic view of the barnase reporter assay. 55

Figure 4.1: Pairwise sequence alignment with PylS from M. mazei and M. barkeri. 57

Figure 4.2: Crystal structure of the catalytic domain of PylS from M. mazei. 58

Figure 4.3: Cm-Assay with pBK PyIS His ${ }_{6}$-tag variants. 59

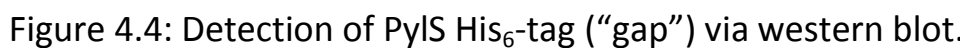
59

Figure 4.5: Truncations in the PyIS "gap" ("wt") variant to investigate the effect on the suppression efficiency. 60

Figure 4.6: Cm-Assay with truncation variants of PylS "gap" ("wt"). 61

Figure 4.7: Predicted secondary structure of PylT. 62

Figure 4.8: Establishing northern blots to detect specific tRNAs. 63

Figure 4.9: Detection of PyIT in total RNA extracts 64

Figure 4.10: Crystal structure of tyrosyl-tRNA synthetase from M. jannaschii complexed with tRNA(Tyr) and L-tyrosine. 65

Figure 4.11: Cm-Assay with pBK MjYRS $\mathrm{His}_{6}$-tag variants. 66

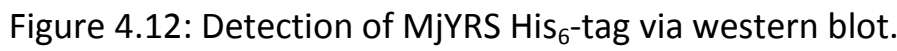
66

Figure 4.13: Predicted secondary structure of MjYT_CUA. 
Figure 4.14: Detection of MjYT_CUA in total RNA extracts. 68

Figure 4.15: Sequence alignment of MjYT 69

Figure 4.16: Refinement of MjYT detection 70

Figure 4.17: Schematic overview of the synthetic promoter libraries. 71

Figure 4.18: Cm-Assay with single colonies from PylS library. 73

Figure 4.19: Comparative analysis of PylS lib clones via western and northern blot. 74

Figure 4.20: Inverse gradient PCR for the MjYRS promoter library 75

Figure 4.21: Cm-Assay with single colonies from MjYRS_AGGA library. 76

Figure 4.22: Suppression efficiency of MjYRS_AGGA lib clones. 77

Figure 4.23: Cm-Assay with single colonies from PyIT library. 79

Figure 4.24: Cm-Assay with single colonies from MjYT_UCCU library. 79

Figure 4.25: Comparative analysis of defined standard promoters with WT gInS and library clone $3 \mathrm{E}$ promoter.

Figure 4.26: Schematic overview of the modular genetic tool. 85

Figure 4.27: Cm-Assay with final pBK PylS plasmid and intermediate steps from preparation for the modular genetic tool.

Figure 4.28: Cm-Assay to detect the stage responsible for PylS activity loss. 87

Figure 4.29: $\mathrm{Cm}$-Assay of the PylS Ndel restore mutants. 88

Figure 4.30: Restriction digests of the final modular genetic tool. 89

Figure 4.31: Comparison of the modular genetic tool to the four plasmid system. 91

Figure 4.32: Cm-Assay for the background reduction of combined PylS and PylT promoter library.

Figure 4.33: Restriction digests of the modular genetic tool with wild type and library promoters. 
Figure 4.34: FACS to identify the best combination of all 4 libraries on the Genetic Tool at once (GT4L). 95

Figure 4.35: Comparison of the modular genetic tool (GTF) to single four library plasmid clones. 99

Figure 4.36: Comparison of the standard modular genetic tool to variants with defined promoters. 101

Figure 4.37: Small scale expression and purification of GST-MBP comparing different genetic tool variants and the 4 plasmid system. 102

Figure 4.38: Growth curve and GFP fluorescence measurement comparing different genetic tool variants and the 4 plasmid system. 103

Figure 5.1: Cm-Assay with the 20 best clones from the MjAzFRS library. 105

Figure 5.2: Incorporation of AzF into GFP and H3 using MjAzFRS library clone 17 (pCLA149). 106

Figure 5.3: Incorporation of BCNK and NorK into myoglobin. 108

Figure 5.4: Incorporation of NorK into histone H3. 109

Figure 5.5: Labeling of histone H3 for (pseudo) FRET.

Figure 5.6: Labeling of histone H3 with four different tetrazine conjugated fluorophores. 111

Figure 5.7: Incorporation of Bock and NorK into NbSyn2.

Figure 5.8: Fluorescently labeled NbSyn2

Figure 5.9: Incorporation of BocK into NbGFP

Figure 5.10: sfGFP expression for in-cell NMR measurements.

Figure 5.11: Arabinose inducible promoter for the orthogonal translational machinery... 116

Figure 5.12: Effect of spectinomycin on sfGFP expression.

Figure 5.13: sfGFP expression with selected spectinomycin concentrations. 118

Figure 8.1: Sequence alignment of selected PylS promoter library clones. 158

Figure 8.2: Sequence alignment of selected MjYRS_AGGA promoter library clones. 159 
Figure 8.3: Sequence alignment of selected PyIT promoter library clones. ........................ 160

Figure 8.4: Sequence alignment of selected MjYT_UCCU promoter library clones. ............ 161

Figure 8.5: Effect of spectinomycin on in-cell fluorescence of sfGFP.............................. 162

Figure 8.6: Titration of spectinomycin to optimize in-cell fluorescence of sfGFP.............. 163 


\section{List of Tables}

Table 3.1: Overview of antibiotics used in growth media/agar plates

Table 3.2: Overview of growth media used for culturing of $E$. coli......

Table 3.3: Overview of unnatural amino acids used for genetic code expansion

Table 3.4: Overview of enzymes used

Table 3.5: Overview of cell lines used.

Table 3.6: Overview of commonly used buffers \& solutions

Table 3.7: Overview of fluorescent dyes used for protein labeling

Table 3.8: Overview of antibodies used for immuno blotting (western \& northern blots). . 28

Table 3.9: Composition of polyacrylamide gels for SDS PAGE

Table 3.10: Pipetting scheme for standard test and preparative digests

Table 3.11: Percentages of agarose gels depending on the size of the DNA (fragments).... 40

Table 3.12: Pipetting scheme for a standard ligation reaction mix

Table 3.13: Standard course of a PCR

Table 3.14: Pipetting scheme for the composition of one PCR reaction mix

Table 3.15: Pipetting scheme for the composition of one QuikChange PCR reaction mix.... 44

Table 3.16: Standard course of a QC-PCR

Table 3.17: Pipetting scheme for the composition of one inverse PCR reaction mix......

Table 3.18: Standard course of a restriction enzyme-mediated inverse PCR.

Table 3.19: Pipetting scheme for the composition of one sequencing PCR reaction mix..... 48

Table 3.20: Standard course of a sequencing PCR

Table 3.21: Composition of an acid urea polyacrylamide gel for acid urea PAGE 50

Table 3.22: Pipetting scheme for the composition of one transcription reaction mix. 
Table 3.23: Overview of RNA transcripts made by T7 RNA polymerase.

Table 4.1: Relative in vivo strength of selected promoters.

Table 4.2: Overview of all QuikChanges performed in order to construct the modular genetic tool.

86

Table 4.3: Overview of preparative digests performed in order to construct the modular genetic tool. 89

Table 4.4: Cm-Assay for the sorting of the four library plasmids. 97

Table 5.1: Amino acid sequence of the 20 best clones from the MjAzFRS library. 106

Table 8.1: List of plasmids. 142

Table 8.2: List of oligos (primers and probes). 146

Table 8.3: Details of the construction of plasmids made during this thesis. 152 


\section{Danksagungen}

An dieser Stelle möchte ich einigen Leuten danken, die entweder durch ihre fachliche Kompetenz und/oder ihre Freundschaft entscheidend zum Gelingen dieser Arbeit beigetragen haben:

In erster Linie möchte ich mich bei Herrn Jun.-Prof. Dr. Heinz Neumann bedanken, der mir die Möglichkeit gegeben hat, meine Doktorarbeit in seiner Abteilung in dem spannenden Themengebiet der synthetischen Biologie anzufertigen. Ich danke ihm für sein entgegengebrachtes Vertrauen und die kontinuierliche Unterstützung während der gesamten Zeit und hoffe einiges von seinem Ideenreichtum mitgenommen zu haben.

Prof. Dr. Jörg Stülke danke ich für die Übernahme des Korreferats und dafür, dass er auch weit über meine Diplomarbeit hinaus immer ein offenes Ohr hatte und mir mit Rat und Tat freundschaftlich zur Seite stand.

A special "thank you" goes to Dr. Bryan Wilkins. I am really thankful that he proofread my thesis although he already read Svenja's thesis and was himself that busy.

Des Weiteren danke ich sowohl der AG Neumann, als auch der AG Ficner, für die entspannte Arbeitsatmosphäre, für hilfreiche Diskussionen und auch für manch zünftige (Weihnachts-)Feiern ;).

Ein besonderer Dank gilt auch Christian Hoffmann, der mich an so manch einem extrem langen Arbeitstag bis in die Nacht begleitet und mit unzähligen Ideen und Tipps unterstützt hat, aber auch für die netten Abende außerhalb der Arbeit.

Meinen Bachelorstudenten Miguel Sánchez und Julia Motz bin ich dankbar für ihre erfolgreiche Mithilfe bei einem Teilprojekt dieser Arbeit.

Auch unter der weisen Voraussicht, dass er es wahrscheinlich niemals lesen wird, bedanke ich mich bei meinem ehemaligen Biologielehrer Herrn Veddeler, ohne den ich wohl niemals das Biologiestudium angestrebt hätte.

Meinen Freunden/Kommilitonen vor Ort (im Besonderen seien hier Arne Schmeisky und Marcel Kunadt genannt) danke ich für gesellige Abende und die damit verbundene Ablenkung, wenn mal nicht alles geradeaus lief. 
Meinen Freunden zu Hause, besonders den „Oldenburgern“, sei für ihre nun teilweise schon weit über ein Jahrzehnt andauernde Freundschaft gedankt und die damit verbundene Unterstützung, die letztlich auch maßgeblich zum Gelingen dieser Arbeit beigetragen hat.

Bei meiner Freundin Corinna Krüger möchte ich mich ebenfalls ganz besonders bedanken, für die bereits gemeinsam verbrachte Zeit und für die, die noch folgen wird. Ich danke ihr, dass sie mich so weit es möglich war unterstützt und entlastet hat, mit mir durch die Höhen und Tiefen beim Anfertigen der Arbeit gegangen ist und mir somit das Schreiben um ein Vielfaches erleichtert hat.

Zu guter Letzt, aber definitiv nicht weniger als den zuvor genannten, danke ich meiner Familie und vor allem meinen Eltern Gert und Angelika. Euer steter Glauben an mich und eure kontinuierliche Unterstützung, die nicht nur finanzieller sondern auch seelischer und moralischer Natur war, hat mir geholfen zu erreichen, wo und wer ich heute bin. 


\section{Abbreviations}

$\mu \mathrm{g}$

$\mu \mathrm{L}$

$\mu \mathrm{M}$

$\mathrm{AA}$

aaRS/RS

$A G$

Amp

AP

APS

Ara

AzF

BCNK

BME

Boc

Bock

bp

BPA

BSA

CAT

Ch.

CIP

$\mathrm{Cm}$ microgram

microliter

micromolar

amino acid

aminoacyl-tRNA synthetase

Arbeitsgruppe

ampicillin

alkaline phosphatase

ammonium persulfate

arabinose

4-azido-L-phenylalanine

bicyclo[6.1.0]non-4-yn-9-ylmethanol-L-lysine

$\beta$-mercaptoethanol

tert.-butyl

$\mathrm{N}(\varepsilon)$-tert.-butyl-oxycarbonyl-L-lysine

base pair(s)

p-benzoyl-L-phenylalanine

bovine serum albumin

chloramphenicol acetyltransferase

chapter

calf intestinal alkaline phosphatase

chloramphenicol 


\begin{tabular}{|c|c|}
\hline ddNTP & dideoxynucleotide triphosphate \\
\hline DIG & digoxigenin \\
\hline DMSO & dimethyl sulfoxide \\
\hline DNA & deoxyribonucleic acid \\
\hline dNTP & deoxyribonucleotide triphosphate \\
\hline DTT & dithiothreitol \\
\hline E. coli & Escherichia coli \\
\hline e.g. & exempli gratia \\
\hline $\mathrm{ECL}$ & enhanced chemiluminescence \\
\hline EDTA & ethylenediaminetetraacetic acid \\
\hline et al. & et alii/aliae/alia \\
\hline $\mathrm{EtOH}$ & ethanol \\
\hline FACS & fluorescence-activated cell sorting \\
\hline FRET & Förster/fluorescence resonance energy transfer \\
\hline g & gram \\
\hline$g$ & gravitational acceleration \\
\hline GFP & green fluorescent protein \\
\hline GST & glutathione $S$-transferase \\
\hline His & histidine \\
\hline HRP & horseradish peroxidase \\
\hline i.e. & id est \\
\hline IPTG & isopropyl $\beta$-D-1-thiogalactopyranoside \\
\hline Kan & kanamycin \\
\hline 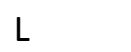 & liter \\
\hline
\end{tabular}


LB

lib

M

M. barkeri (Mb)

M. jannaschii (Mj)

M. mazei

MBP

MCS

$\mathrm{MeOH}$

mg

$\min$

MjYRS

MjYT

$\mathrm{mL}$

$\mathrm{mM}$

Myc

$\mathrm{Nb}$

NB

$\mathrm{nm}$

$\mathrm{nM}$

Nork

nt

$\mathrm{OD}_{600}$

O-mRNA lysogeny broth

library

molar

Methanosarcina barkeri

Methanocaldococcus jannaschii

Methanosarcina mazei

maltose binding protein

multiple cloning site

methanol

milligram

minute

M. jannaschii tyrosine aminoacyl-tRNA synthetase

M. jannaschii tyrosine tRNA

milliliter

millimolar

myelocytomatosis

nanobody

northern blot

nanometer

nanomolar

NE-5-norbornene-2-yloxycarbonyl-L-lysine

nucleotide(s)

optical density at $600 \mathrm{~nm}$ wavelength

orthogonal mRNA 


\begin{tabular}{|c|c|}
\hline ORBS & orthogonal ribosomal binding site \\
\hline O-ribosome & orthogonal ribosome \\
\hline PAGE & polyacrylamide gel electrophoresis \\
\hline PBS & phosphate buffered saline \\
\hline PCR & polymerase chain reaction \\
\hline Pfu & Pyrococcus furiosus \\
\hline $\mathrm{PIC}$ & protease inhibitor cocktail \\
\hline PMSF & phenylmethanesulfonyl fluoride \\
\hline PVDF & polyvinylidene difluoride \\
\hline Pyls & pyrrolysine aminoacyl-tRNA synthetase \\
\hline PylT & pyrrolysine tRNA \\
\hline QC & QuikChange \\
\hline RNA & ribonucleic acid \\
\hline rNTP & ribonucleotide triphosphate \\
\hline rpm & revolutions per minute \\
\hline RT & room temperature \\
\hline SAP & shrimp alkaline phosphatase \\
\hline SDS & sodium dodecyl sulfate \\
\hline sm & single-molecule \\
\hline Sm & spectinomycin \\
\hline SSC & saline-sodium citrate \\
\hline T7RP & T7 RNA polymerase \\
\hline$T a q$ & Thermus aquaticus \\
\hline ТВ & TRIS-Borate-EDTA buffer \\
\hline
\end{tabular}


TBS

TCA

TCEP

TEMED

Tet

TRIS

tRNA

UAA

UV

v

w

WB

WT
TRIS buffered saline

trichloroacetic acid

tris(2-carboxyethyl)phosphine

$\mathrm{N}, \mathrm{N}, \mathrm{N}^{\prime}, \mathrm{N}^{\prime}$-tetramethylethylenediamine

tetracycline

tris(hydroxymethyl)aminomethane

transfer RNA

unnatural amino acid

ultraviolet

volume

weight

western blot

wild type 


\section{Summary}

Proteins are composed of 20 canonical amino acids whose unique arrangements predefine a protein's structure and function. Importantly, most proteins are not static conformations but rather very dynamic entities that undergo various structural modifications under different "stimuli". The comprehension of these dynamic processes is necessary to understand how proteins work.

Förster/Fluorescence resonance energy transfer (FRET) became a powerful tool to investigate conformational changes of proteins, and recent advances in technology haven given the capability for studies even on a single-molecule $(\mathrm{sm})$ level. Therefore precise labeling of the proteins with suitable fluorophores is essential, however, remains a challenging task at present. Although chemical bioconjugation of fluorophores and proteins work, more or less reliably, specificity is a drawback for longer polypeptides and full-length proteins.

To overcome the issue of specificity, synthetic biologists have opened new avenues by developing an expansion of the genetic code. This technique requires the introduction of exogenous nonsense suppressor tRNAs and their cognate aminoacyl-tRNA synthetases (aaRS) into the host cell, that have to work completely orthogonal to the endogenous components. This allows the incorporation of additional "unnatural" amino acids (UAAs) into proteins at the genetic level. These UAAs can bear many different functional groups with unique chemical or biophysical properties.

Since we were interested in introducing two fluorophores, site-specifically into a protein, we had to use two tRNA/aaRS pairs, along with the plasmid harboring the gene of interest. This general approach necessitated multiple plasmids with different antibiotic resistances leading to heightened stress in the host cells. Additionally, using two non-cognate tRNA/aaRS pairs displayed toxic side-effects and required balanced levels within cells. Moreover, the two different UAAs drastically decreased the suppression efficiency and, in turn, the expression levels of protein. Therefore, an overall optimization of the system was essential.

Herein, we describe the optimization process. We set out to reduce the number of plasmids used in this system, resulting in a highly modular genetic tool. We designed this system to allow for easy exchange with other tRNA/aaRS pairs to introduce new UAAs. We explored 
promoter libraries to fine-tune the expression levels of tRNA/aaRS pairs, which had profound effects on the UAA incorporation efficiency.

Using the above system we achieved higher levels of protein expression with two different UAAs and are currently establishing bioorthogonal labeling strategies for use in smFRET studies.

As a result of the work for the present thesis the following article was submitted to "Chembiochem: a European journal of chemical biology" and will be published soon:

Lammers, C.; Hahn, L. E. \& Neumann, H. "Optimized plasmid systems for the incorporation of multiple different unnatural amino acids by evolved orthogonal ribosomes." Chembiochem, DOI: 10.1002/cbic.201402033 (2014). 


\section{Introduction}

\subsection{Proteins Encoded By The Genetic Code}

The three domains of (cellular) life proposed by Woese ${ }^{[1]}$ imply millions of discovered species, as well as those yet undiscovered ${ }^{[2,3]}$. Despite this huge diversity, all living organisms, whether they are single or multi-celled, have common similarities. They are all built upon simple organic compounds, such as nucleotides and amino acids, the biological "bricks of life". These monomeric subunits form larger polymeric macromolecules, including DNA/RNA and proteins respectively, assembled in intricate networks of biological processes.

Proteins play a leading part in these highly complex biological systems since they participate in virtually all processes, making them one of the most studied objects in life science. Their variety in structure and function is determined by unique arrangements of the 20 canonical amino acids. Minor mutations, even a single amino acid, can lead to altered conformations that perturb the native function of the protein. The individual amino acid arrangement is dictated by the nucleotide sequence of a protein's gene, encoded by non-overlapping triplet codons, composed of a combination of the four bases adenine (A), cytosine $(C)$, guanine $(G)$ and thymine $(T)$. This genetic code consists of 64 possible codons from which 61 are assigned to the 20 amino acids and the remaining three to the termination of protein synthesis ${ }^{[4-11]}$

The assembly of proteins is performed by ribosomes, huge macromolecular complexes composed of ribosomal $(r)$ RNAs and proteins divided into small and large subunits. The process of decoding the mRNA into proteins is called translation and can be separated into the four steps. These are termed initiation, elongation, termination, and recycling ${ }^{[12,13]}$. Protein synthesis in prokaryotes and eukaryotes is different but primarily they differ only in the initiating steps ${ }^{[14]}$.

Translational events in the prokaryote Escherichia coli (E. coli) starts with the complex formation between initiation factors IF1 and IF3, as well as the small (30S) ribosomal subunit ${ }^{[15,16]}$. This complex is able to bind cellular mRNAs by the interaction of the antiShine-Dalgarno sequence on the 16S rRNA and the Shine-Dalgarno sequence $\left(5^{\prime}\right.$-AGGAGG-3') on the mRNA, located upstream of an AUG start codon ${ }^{[17,18]}$. The mRNA is guided by this sequence and is thereby positioned in the $\mathrm{P}$ site of the ribosome. The recruitment of the GTP-binding protein IF2 and the initiator tRNA, aminoacylated with 
formylated methionine (fMet-tRNA), prepares the $30 \mathrm{~S}$ initiation complex for the association with the large (50S) subunit of the ribosome upon GTP hydrolysis. The newly formed 70S initiation complex is poised for the translational elongation phase ${ }^{[13-15]}$.

During elongation, adding amino acids in recurring steps extends the nascent polypeptide chain. Therefore, ternary complexes composed of elongation factor Tu (EF-Tu), GTP and tRNA, which has been previously aminoacylated with the correct amino acid by a cognate aminoacyl-tRNA synthetase $(\mathrm{aaRS})^{[19,20]}$, are delivered into the decoding center within the A site of the ribosome ${ }^{[21]}$. The ribosome actively monitors the selection of the proper aminoacyl-tRNA, which is dictated by the mRNA codon, and thus maintains the fidelity of the genetic code ${ }^{[22-24]}$. Whereas the first two codon-anticodon base pairs have to form strict Watson-Crick pairs ( $\mathrm{A}-\mathrm{U}$ and $\mathrm{G}-\mathrm{C}$ ), excluding non-cognate and wobble pairs, wobble pairing at the third position of the codon is tolerated ${ }^{[23,25]}$. This means, the $5^{\prime}$-base of the tRNA anticodon can form non-Watson-Crick base pairs with the $3^{\prime}$-base of the mRNA codon, permitting some tRNAs to decode more than one codon. The delivery of the appropriate ternary complex into the ribosome's A site facilitates GTP hydrolysis by EF-TU, followed by its dissociation from the ribosome and the accommodation of the aminoacylated tRNA acceptor stem in the peptidyl transferase center (PTC) within the large subunit for peptide bond formation ${ }^{[13,24]}$. This causes the transfer of the nascent peptide chain from the $P$ site to the A site tRNA. To remove the deacylated tRNA in the P site and to position a new codon in the A site for the arrival of another aminoacyl-tRNA in the next round, the elongation cycle ends with the translocation of the tRNAs and the mRNA by precisely one codon. This process is catalyzed by the elongation factor EF-G at the cost of GTP hydrolysis ${ }^{[13,26]}$.

The elongation phase terminates when one of the three stop codons (UAA, UAG or UGA) moves into the $A$ site. These codons are recognized by the release factors RF1 (UAA, UAG) and RF2 (UAA, UGA) ${ }^{[27,28]}$ that mediate the cleavage of the nascent polypeptide chain from the tRNA bound in the ribosome's P site, leading to the release of the newly synthesized protein from the ribosome. Afterwards, the GTP-binding protein RF3 accelerates the dissociation of RF1/RF2 from the ribosome, which is subsequently recycled for the translation of further proteins ${ }^{[13]}$. 


\subsection{Expansion And Modification of The Genetic Code}

The genetic code of all living organisms is near-universally conserved and was long concidered to be restricted to the 20 naturally occurring amino acids. In 1986 two workgroups independently discovered that the nonstandard amino acid selenocysteine $(\mathrm{Sec})$ is directly incorporated into proteins in response to in-frame opal stop codons (UGA), instead of being created by posttranslational modification ${ }^{[29,30]}$. Afterward, this system was regarded as an expansion of the genetic code and selenocysteine was titled the $21^{\text {st }}$ amino $\operatorname{acid}^{[31,32]}$. Sixteen years later pyrrolysine $(\mathrm{Pyl})$ was found to be the $22^{\text {nd }}$ genetically encoded amino acid, this time in response to the amber stop codon (UAG) ${ }^{[33-35]}$. Whereas Sec is present in prokaryotes and eukaryotes ${ }^{[36]}$, the distribution of Pyl appears limited to the Methanosarcinacea and Gram-positive Desulfitobacterium hafniense ${ }^{[37]}$. Furthermore, both amino acids differ in their aminoacylation mechanism. Sec is made via an enzymatically modified serine that was charged to a special selenocysteinyl-tRNA. In contrast, Pyl is directly paired to pyrrolysyl-tRNA (PyIT) by the cognate aminoacyl-tRNA synthetase PylS ${ }^{[37-}$ 39].

In addition to Sec and Pyl, even more deviations have been found to the standard genetic code. Genome analyses revealed ten codon reassignments in prokaryotic and eukaryotic nuclear codes which all are a subset of 16 changes occurring in mitochondrial $\operatorname{codes}^{[40]}$. Moreover, some methanogenic archaea compensate the lack of a canonical cysteinyl-tRNA synthetase by a particular pathway using $O$-phosphoserine that is enzymatically converted to cysteine prior to incorporation into a nascent protein ${ }^{[40]}$. All these modifications show a certain flexibility of the genetic code towards evolutionary novelties, giving the potential for additional genetically encoded nonstandard amino acids that might exist in stilluncharacterized genomes ${ }^{[40]}$. However, the search for the $23^{\text {rd }}$ amino acid has not yet been successful, making the appearance of further widely spread amino acids improbable ${ }^{[41]}$.

At the end of the last century, scientists began to exploit the degeneracy of the genetic code, in order to artificially expand it for the genetically encoded incorporation of amino acids with new functionalities into proteins. These "unnatural" amino acids (UAAs) bear many different functional groups, such as posttranslational modifications, UV-inducible crosslinkers, spectroscopic probes and chemical handles that can be modified chemically, even in living cells ${ }^{[42]}$. This required the introduction of exogenous tRNAs and their cognate aaRSs into the host cell, which have to work completely orthogonal to the endogenous components. That means, the introduced tRNAs should not be charged with any canonical amino acid by the host's aaRSs and, in turn, the orthogonal aaRS should not aminoacylate 
any endogenous tRNA with UAAs. The anticodon of the orthogonal tRNA is typically complementary to blank (nonsense, frameshift, or otherwise unused) codons, especially the rarely used amber stop codon, allowing the reassignment of the appropriate codon to the amino acid used as a substrate by the orthogonal aaRS. The feasibility of this method was proven in 1998 by Furter who was able to site-specifically incorporate p-fluorophenylalanine ( $p$-F-Phe) into dihydrofolate reductase (DHFR) in E. coli expressing a yeast amber suppressor tRNA/phenylalanyl-tRNA synthetase (PheRS) pair ${ }^{[43]}$.

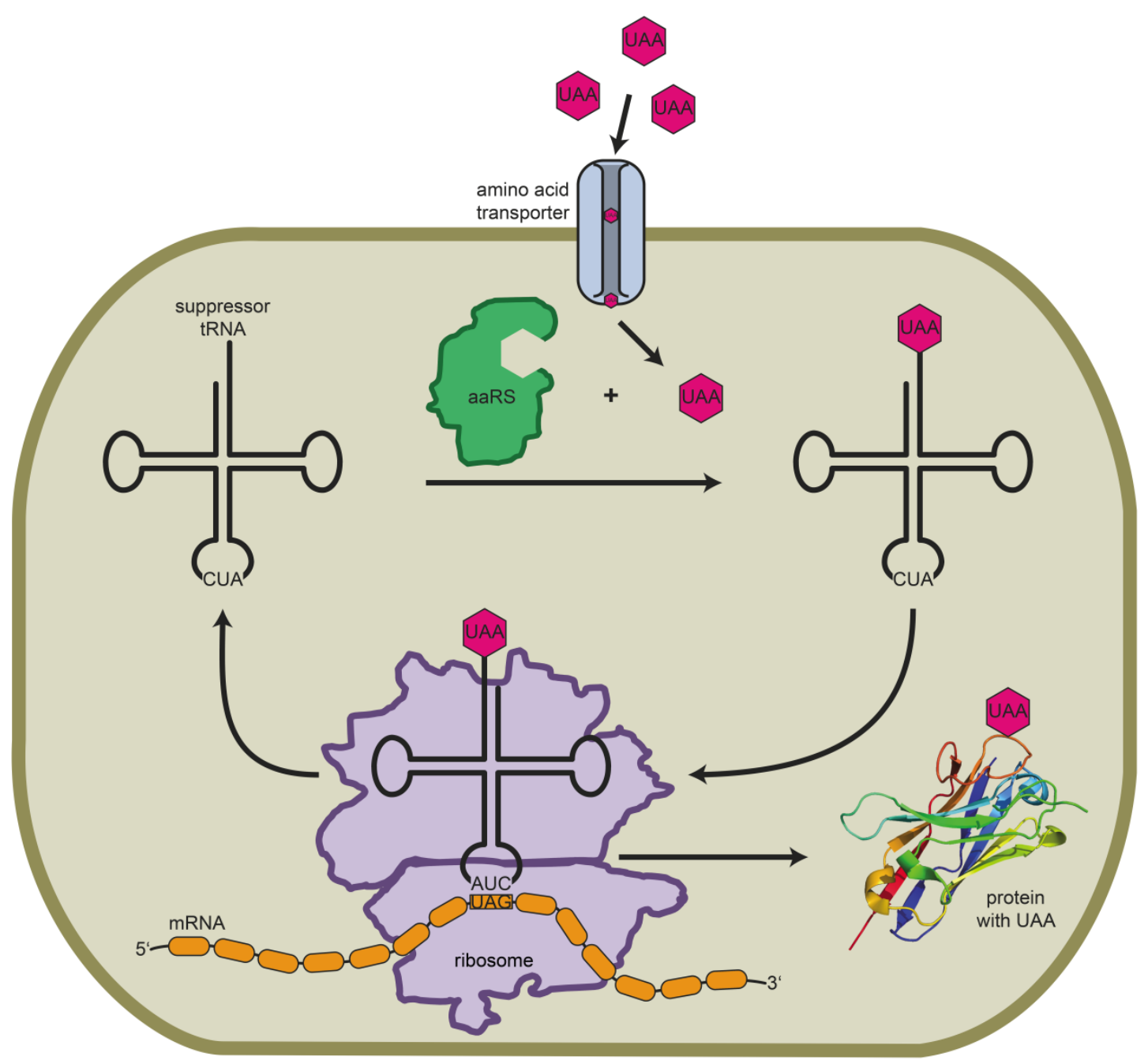

Figure 2.1: Schematic view for genetic code expansion.

Desired unnatural amino acids (UAAs) are taken up by the cell using endogenous transporters. These UAAs are used by evolved aminoacyl-tRNA synthetases (aaRSs) to charge corresponding evolved tRNAs. The tRNAs are then used by the ribosomes to decode (mostly) amber codons introduced in the mRNA, to incorporate the UAAs at predetermined sites on the protein of interest.

Whereas the yeast PheRS accepted the substrate analogue $p$-F-Phe without further modifications, advances in this system necessitated the adaption of aaRS' specificities towards specific UAAs ${ }^{[44]}$. Furthermore, the original standard amino acid phenylalanine was still a substrate for the PheRS, resulting in non-homogenously labeled protein. This 
disadvantage was overcome by a large excess (up to 30 -fold) of $p$-F-Phe supplementation in the growth medium ${ }^{[43]}$. The first tRNA/aaRS pair that was truly orthogonal and only recognizing the desired UAA was derived from the tyrosyl pair from Methanococcus jannaschii (M. jannaschii) in the workgroup of Peter Schultz. This pair was evolved to incorporate $O$-methyl-L-tyrosine into DHFR in response to an amber codon ${ }^{[45]}$ (Figure 2.1). To achieve this, they developed a systematic approach to alter the specificity of a synthetase for a certain UAA. First, all active-site residues interacting with the actual tyrosine substrate were randomly mutated, yielding a large library of synthetase variants which were passed through multiple rounds of stringent positive and negative selection. The positive selection was based on a reporter plasmid containing an antibiotic resistance gene with amber mutations at permissive sites, in this case a chloramphenicol acetyltransferase (CAT). E. coli cells simultaneously transformed with this reporter and the aaRS library plasmids were only able to survive in media containing chloramphenicol and the UAA, if they harbored a functional synthetase variant recognizing either the UAA or a natural amino acid (also see Ch. 3.2.4.1). The subsequent negative selection eliminated undesired aaRSs suppressing amber codons with endogenous amino acids in the absence of the UAA. Therefore, active synthetase clones from the first round were combined with a reporter plasmid comprising an amber mutant of the toxic barnase gene. Clones that produce full-length barnase protein using canonical amino acids will die because of its ribonuclease activity, which is toxic to cells without its specific inhibitor barstar ${ }^{[46]}$ (also see Ch. 3.2.4.3). Multiple rounds of these two selections were performed, leading to an orthogonal, highly specific and amber suppressing aaRS variant ${ }^{[44]}$ (Figure 2.2).

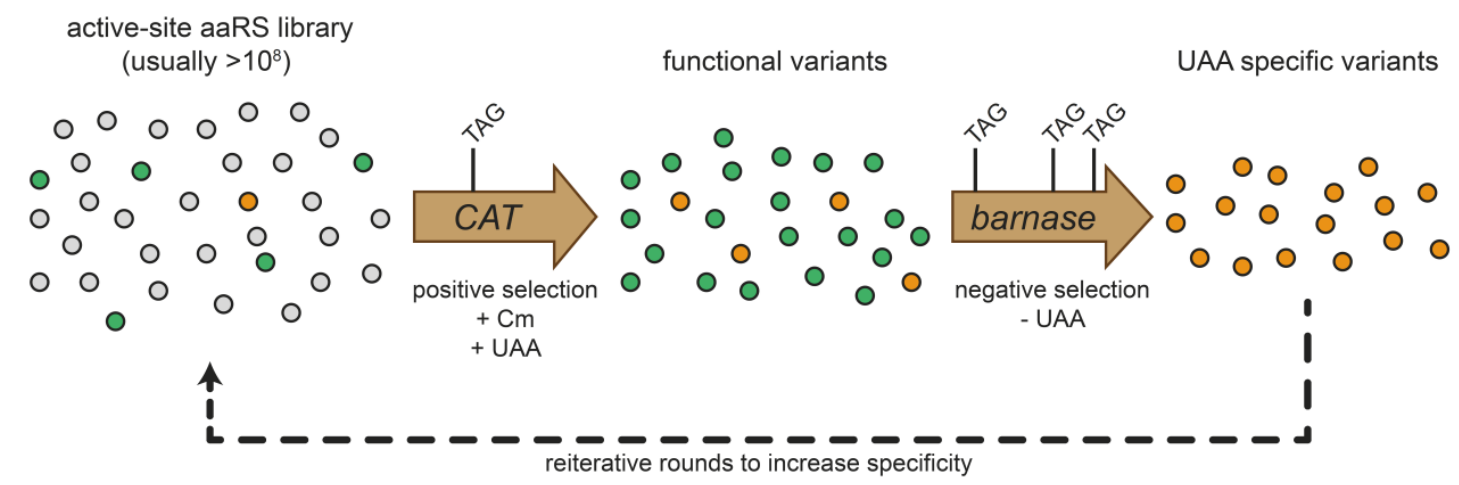

Figure 2.2: General selection scheme for the evolution of aminoacyl-tRNA synthetase (aaRS) substrate specificities for unnatural amino acids (UAAs) ${ }^{[44]}$.

First, non-functional aaRS library variants are removed in a positive selection in the presence of the UAA and chloramphenicol $(\mathrm{Cm})$. Functional variants suppress the amber codon within the chloramphenicol acetyltransferase (CAT) gene with both natural and unnatural amino acids. Synthetases specific for the UAA are isolated in a negative selection in the absence of the UAA, while suppression of the amber codons in the barnase gene leads to cell death. 
The success in E. coli led to the development of a very similar selection approach in yeast, driven by amber suppression in the transcriptional activator GAL4. The production of fulllength GAL4 in the presence of the UAA activates the expression of GAL4-responsive HIS3, URA3, and lacZ reporter genes, allowing for survival on media lacking histidine or uracil. Negative selections to remove unspecific synthetases are based on the conversion of the protoxin 5 -fluoroorotic acid (5-FOA) to its toxic product by the URA3 gene product on media without the $U A A^{[44,47]}$.

The evolution of tRNA synthetases in cells of higher eukaryotes, like mammalians, is more difficult due to technical issues concerning transformation efficiency, slow doubling times, and growth conditions. In this direction, a shuttle approach was applied with tRNA/aaRS pairs, which are orthogonal both in E. coli or Saccharomyces cerevisiae (S. cerevisiae) and in mammalian cells. Pairs evolved for UAAs in the easier to handle bacterium or yeast can then be transferred into mammalian cells while keeping orthogonality ${ }^{[48]}$.

Using the aforementioned techniques a variety of orthogonal tRNA/aaRS pairs from various organisms have been used to add up to 100 unique unnatural amino acids to the genetic code of prokaryotes, including E. coli and some mycobacteria, and eukaryotes, like the yeasts S. cerevisiae and Pichia pastoris and even the multicellular organisms Caenorhabditis elegans and Mus musculus ${ }^{[42,48]}$. However, the majority of all genetic code expansion approaches were performed with only four different tRNA/aaRS pairs, each suitable for a particular model organism. First, the already mentioned M.jannaschii tyrosyl pair (MjYRS/MjYT) is orthogonal only in E. coli and other bacteria. The second and third are two synthetases from E.coli decoding for tyrosine (EcTyrRS) and leucine (EcLeuRS) in combination with their cognate tRNAs and can be utilized only in yeast, mammalian and other eukaryotic cells. Lastly, the aforesaid PyIS/PyIT pair from Methanosarcina species provides the advantage of being orthogonal in both bacteria and eukaryotic cells, showing no cross-reactions with endogenous synthetases or tRNAs. Additionally, the natural aaRS substrate specificity must not be destroyed before the evolution for a new UAA, since it decodes pyrrolysine and none of the 20 canonical amino acids ${ }^{[49]}$.

\subsection{Limitations And Optimizations of The Expanded Genetic Code}

Although the genetically encoded incorporation of UAAs into proteins works, in principle, this system has to cope with a large range of limitations. These restrictions reduce the efficiency or even the feasibility of the system. Not all the desired UAAs exhibit the cell 
permeability required to cross the cell membrane, impeding the uptake by the cell and thereby the integration into the translational apparatus. Even if the UAA can be imported, the structural nature of the aaRS' active site must allow the evolution of its specificity to the particular amino acid. Furthermore, the incorporation efficiency of UAAs at certain sites in the protein can also be negatively influenced by effects of the mRNA context ${ }^{[50]}$ and the local protein structure ${ }^{[51]}$, as well as further factors like protein folding and stability ${ }^{[48]}$.

One of the main reasons for decreased yields of proteins with UAAs is the competition of reassigned codon suppression with other cell activities, such as binding of a release factor to a stop codon or the erroneous recognition of a frameshift codon by three-base anticodon tRNAs. In E. coli suppression of the most commonly used blank codon, the amber codon, is impaired by the interaction with its release factor RF1, leading to truncated proteins. The incorporation of several UAAs into the same protein intensifies this effect. A simple deletion of the RF1-encoding gene, $\operatorname{prfA}$, seemed to be unfeasible since this factor has been reported to be essential ${ }^{[22,53]}$.

However, a couple of workgroups successfully reduced the competing effect of RF1 or even managed to perform a prfA knockout. For instance, enhanced amber suppression efficiency by overexpressing the C-terminus of the ribosomal protein L11, which has been suggested to play an important role in RF1-mediated translation termination, facilitated the incorporation of up to three acetyllysines into the same green fluorescent protein (GFP) ${ }^{[54]}$. Rydén and Isaksson found a temperature sensitive RF1 mutant that showed an increased efficiency to several amber tRNA suppressors ${ }^{[55]}$. This could be utilized to favor UAA incorporation in response to amber codons, but ongoing growth at high temperature would be fatal for the cell and could affect the production of many recombinant proteins adversely.

The Nakamura group was able to compensate for this temperature dependent RF1 growth deficit by engineering RF2 with a single amino acid substitution to an omnipotent release factor $\left(\mathrm{RF}^{*}\right)$, capable to terminate translation at all three stop codons ${ }^{[56]}$. The use of RF2* even permitted a chromosomal RF1/RF2 double knockout, indicating that RF1 is nonessential in E. coli. This hypothesis was emphasized by the work of Wang and coworkers who showed the feasibility of a prfA deletion strain by "fixing" the RF2-encoding prfB gene. The engineering of their RF2 variant $\left(p r f B^{f}\right)$ included the removal of an in-frame UAG autoregulation element and the reversion of a peculiar mutation (A246T) that only occurs in E. coli strains derived from K-12, impairing RF2's release activity for the ochre (UAA) codon by a factor of five ${ }^{[57]}$. The reduced activity of mutated RF2 was supposed to be 
the reason for the apparent RF1 essentiality. Indeed, later experiments confirmed that previously reported attempts to knock out RF1 failed because they used E. coli K-12 strains containing the peculiar A246T mutation. Deletion trials using three common E. coli B strains (REL606, BL21, and BL21(DE3)), derived from the second progenitor of most E. coli strains and encoding wild type RF2, allowed successful prfA knockout. Thus, RF1 was proposed to be nonessential for wild type E. coli with nonmutated RF2 ${ }^{[58]}$. The employment of RF1 knockout strains with either wild type background (B strains) or the "fixed" RF2 facilitated the genetically encoded incorporation of up to ten identical UAAs into the same protein.

The workgroups of Yokoyama and Sakamoto accomplished the deletion of prfA while leaving RF2 untouched. They used a combination of a bacterial artificial chromosome, harboring the seven essential open reading frames (ORFs) of $E$. coli naturally ending with an UAG codon but replaced by UAA, and an amber suppressor tRNA. This kept the cells viable and permitted the UAG-dependent incorporation of up to ten natural or six unnatural amino acids into the same polypeptide ${ }^{[59]}$. The role of the amber suppressor tRNA was supposed to alleviate the significantly impaired fitness observed for all mentioned RF1 knockout strains. Its UAG-decoding activity was thought to prevent ribosome stalling at UAG codons and to allow the expression of the nonessential ORFs ending with an amber codon which are then terminated by naturally occurring in-frame "backup" stop codons, opal or ochre, downstream of the suppressed one. Stalled ribosomes have at least three disadvantages that reduce a cell's fitness. First, they cannot finish required protein synthesis. Second, if stalled they are "trapped" to the particular mRNA and cannot be recycled to translate further mRNAs. Third, the tmRNA surveillance system ${ }^{[60,61]}$ is activated, a rescue mechanism responsible for cleavage of the appropriate mRNA and tagging of the premature protein for degradation. Degradation of an overwhelming number of proteins can induce cell death ${ }^{[57,59]}$. Indeed, Yokoyama and Sakamoto could show in following experiments that an efficient decoding of the amber codon, using a tRNA variant with enhanced suppression activity, instead of reducing the number of UAGs in the genome improved the growth of a prfA-deficient strain of $E$. coli ${ }^{[62]}$.

By contrast, the laboratories of Church and Isaacs used multiplex automated genome engineering (MAGE) ${ }^{[63]}$ to create an E. coli strain that had all known UAG stop codons replaced with synonymous UAA codons ${ }^{[64]}$. Afterwards, they deleted RF1 in this strain, reassigning UAG as a sense codon, and compared their approach with those reported by Wang as well as Yokoyama and Sakamoto. They found that their strain was the only one without deleterious effects causing impaired fitness. Furthermore, they proposed that RF1 
is essential only for UAG translational termination and not for UAA termination or other essential cellular functions, since RF2 was kept unmodified ${ }^{[65]}$.

Chin and co-workers pursued an alternative strategy, which did not focus on deletion or mutation of the release factors. They utilized orthogonal ribosomes (O-ribosomes) to reduce the competing effect of RF1. Developed by Chin and Rackham, O-ribosomes form the basis of a parallel and independent translational machinery since their anti-ShineDalgarno sequence was mutated to exclusively bind to the likewise modified ShineDalgarno sequences of orthogonal mRNAs (O-mRNAs). These O-mRNAs, containing an orthogonal ribosomal binding site (ORBS), are in turn, not a substrate for endogenous ribosomes ${ }^{[66]}$. As part of a parallel and independent system O-ribosomes are unburdened from the maintenance of housekeeping gene expression and therefore evolvable to handle new challenges, whereas mutations of the natural ribosome are potentially lethal to the cell. By mutating merely two positions (U531G and U534A) in a loop of the 16S rRNA in the A site, that was found to be in close proximity to both the anticodon of tRNAs bound to mRNA and the RF1, Chin and co-workers created an evolved O-ribosome (termed ribo-X) which showed greatly enhanced suppression of amber codons placed in O-mRNAs. They hypothesized that this improvement resulted from a decreased functional interaction of ribo-X with RF1 ${ }^{[67]}$.

Even in systems optimized for release factor competition an adequate delivery of tRNAs charged with the correct UAA is required. Therefore, the expression of the appropriate tRNAs and their cognate aaRSs have to be carefully balanced, to guarantee an efficient translation but also avoid the overconsumption of biological resources and the generation of toxic side effects by an excessive production of these components. Furthermore, unnecessary metabolic burden for the cell, provoked by, for example, plasmids and corresponding antibiotics, should be prevented. With regard to these issues, several strategies to improve full-length protein yields have been investigated. The simplest solution involved combining the genes encoding the orthogonal tRNA and synthetase on a single plasmid compatible with most expression vectors and strains ${ }^{[68,69]}$. The Schultz laboratory developed a single-plasmid system (pSup) that was consecutively optimized. By merging derivatives of the $M$. jannaschii tyrosyl pair under the control of enhanced promoters (proK instead of Ipp promoter for the tRNA $\mathrm{CUA}_{\text {a }}$ and a strong mutant gInS promoter instead of the wild type variant for the aaRS) on a single vector and raising the tRNA copy number from one to three or six, they observed a 20 -fold ${ }^{[48]}$ increase in the yield of an UAA-containing myoglobin protein in E. coli ${ }^{[69]}$. An improved version of pSup, called 
pSUPAR, comprised an additional copy of the synthetase under the control of an arabinoseinducible ara promoter ${ }^{[70]}$. The replacement of the polycistronic tRNA cluster in PSUPAR with only one copy of an optimized M. jannaschii amber suppressor tyrosyl-tRNA, which has been reported to be generally less toxic to E. coli and to show increased UAA incorporation efficiency with several aaRS ${ }^{[71]}$, resulted in pEVOL. Compared to the first described pSup, pEVOL led to roughly $250 \%$ greater yields of UAA-containing proteins ${ }^{[51]}$. Based on these insights, they finally designed the suppressor plasmid pUltra which harbored a single copy each of the tRNA cassette from pEVOL and the synthetase MjYRS under the control of a tacl promoter. They found pUltra to be better than pEVOL, particularly when tested with weakly expressing mutants or inherently weak suppressor tRNA/aaRS pairs, and achieved the efficient incorporation of up to three UAAs in response to amber stop codons within the same GFP protein.

Since pUltra and pEVOL both have a unique origin of replication and antibiotic resistance marker they are mutually compatible. This was exploited to simultaneously insert two different UAAs into the same protein using amber and ochre codons with pEVOL encoding the $M$. jannaschii amber suppressor pair and pUltra a pyrrolysyl-tRNA/aaRS pair optimized for enhanced ochre suppression. Furthermore, the simple configuration of pUltra enabled the generation of pUltrall, a single plasmid containing both suppression systems for amber and ochre codons. The dual suppressor pUltrall showed similar full-length protein expression levels relative to pUltra and $\mathrm{pEVOL}$ in combination, but reduced the metabolic burden for the cell by one plasmid and thereby one antibiotic ${ }^{[72]}$.

Further efforts to find and optimize factors that affect the incorporation efficiency of unnatural amino acids were made. For instance, in one approach MjYRS was mutated to mediate a better recognition of the cognate tRNA's CUA anticodon ${ }^{[73]}$. Another study described the development of EF-TU mutants that better accept UAAs, particularly those with large side chains or altered backbones ${ }^{[74]}$. But the simultaneous incorporation of two or more distinct UAAs into the same polypeptide poses a difficult challenge.

Each individual UAA necessitates its own orthogonal tRNA/aaRS pair decoding a unique codon. The use of stop codons to generate proteins with multiple UAAs, as reported by Wan et al. ${ }^{[75]}$ or Chatterjee et al. ${ }^{[72]}$ (employing pUltra and pEVOL as described above), quickly reaches its limit. Reassigning some of the 61 other triplet codons encoding natural amino acids would be an option but not easy to handle because this requires genome wide replacements with yet unknown consequences. In addition, even if feasible, this would provide a relatively small set of new blank codons, whereas the usage of codons extended 
to four bases theoretically affords 256 novel blank codons. The combination of a quadruplet and an amber codon has already been successfully utilized to incorporate two unremarkable UAAs into the same model protein ${ }^{[76]}$. However, the capability of natural ribosomes to decode four-base codons with extended anticodon tRNAs is moderate, probably due to poor tRNA accommodation in the ribosome's decoding center ${ }^{[77]}$. This inefficiency cannot be circumvented by evolving the endogenous ribosome because this potentially leads to cell death. For this reason, the Chin laboratory further evolved their orthogonal ribo-X to create an O-ribosome, called ribo-Q1, which exhibits enhanced quadruplet and amber decoding on O-mRNAs. Although they randomly mutated 127 nucleotides of the A site's 16S rRNA, ribo-Q1 contains just two mutations (A1196G and A1197G) with respect to ribo- $X$, allowing an efficient decoding of four-base codons approaching the level of triplet decoding on the natural ribosome ${ }^{[78]}$.

Finally, the number of currently existing mutually orthogonal tRNA/aaRS pairs in bacteria, namely the MjYT/MjYRS pair from M.jannaschii and the PyIT/PylS pair from Methanosarcina species, confines the incorporation of different UAAs into the same polypeptide to two. Since it is not clear if a sufficient number of pairs can be discovered that have been formed by natural evolutionary divergence and which fulfill the absolute required criterion to be orthogonal to both endogenous and already existing exogenous pairs to encode additional unnatural amino acids, alternative strategies need to be invented. One approach was reported by Neumann et al. who designed an orthogonal tRNA/aaRS pair de novo from an existing one by duplicating the MjYT/MjYRS pair in a logical series of mutagenesis and selection steps ${ }^{[79]}$.

\subsection{Applications of The Expanded Genetic Code}

The principles of genetic code expansion haven been applied in numerous studies in order to investigate the nature of a protein itself or to give a protein new properties which are not provided by the common 20 amino acids. Site-specifically incorporated unnatural amino acids offer a myriad of new functionalities, such as posttranslational modifications, UV-inducible crosslinkers, spectroscopic probes and chemical handles that can be subsequently altered, in vitro and in some cases even in vivo ${ }^{[42,48,49]}$. In the following, a small selection of UAAs and their application is presented, exemplifying the enormous potential of an expanded genetic code for basic and applied scientific research. 
To understand how biological processes form life, one has to investigate the participating proteins and their interaction partners. This is sometimes problematic when these interactions are only transient, weak or at particular subcellular locations. Genetically encoded crosslinkers that react with nearby molecules in response to (UV)-light facilitate the covalent trapping of interaction partners for subsequent identification. Several crosslinking systems exist for bacteria, yeast and mammalian cells, including azido derivatives of phenylalanine ${ }^{[47,80]}$, diazirines ${ }^{[81,82]}$ and benzophenones, such as the by far most frequently used $p$-benzoyl-L-phenylalanine $(B P A)^{[47,83]}$. BPA helped to address questions concerning the mechanism of polypeptide translocation across the cytoplasmic membrane in E. coli mediated by the bacterial SecY-SecE-SecG (SecYEG) translocon, a channel-like transmembrane complex, and the SecA ATPase ${ }^{[84,85]}$. Wilkins et al. used BPA to study how histone modifications in yeast influence the complicated but important biological process of chromosome condensation in mitosis ${ }^{[86]}$ and Tagami et al. to define conformational changes in the bacterial RNA polymerase of Thermus thermophilus ${ }^{[87]}$.

A cells' proteome is dynamically controlled by posttranslational modifications (PTMs) that can change a protein's molecular function, affinity for binding partners, localization and stability. However, for the majority of these PTMs the modifying enzymes are still unknown, making the production of homogeneously and site-specifically modified proteins challenging. This can be achieved by using genetic code expansion, which permits the genetically encoded installation of PTMs into proteins, in order to assess the role of a protein's PTMs. So far, a variety of methods have been reported for incorporating nitrated $^{[88]}$ and sulfated ${ }^{[89]}$ tyrosine, analogues of phosphorylated tyrosine ${ }^{[90]}$, phosphorylated serine ${ }^{[91]}$, mono- ${ }^{[92,93]}$ and dimethylated ${ }^{[94]}$ acetylated ${ }^{[95]}$ and ubiquitinated $^{[96]}$ lysine into recombinant proteins. Employing these methods, the effect of H3 K56 acetylation on nucleosome and chromatin structure and function has been examined, revealing that this modification mediated an increased DNA breathing on the nucleosome $\mathrm{e}^{[97]}$. In another case the role of an acetylated lysine in a protein involved in HIV infection, cyclophilin A (СурA), has been identified to regulate key functions of CypA that, among other things, lead to diminished effects of the widely-used immunosuppressive drug cyclosporine $e^{[98]}$.

Furthermore, UAAs with spectroscopic properties have been installed into proteins. For example, the phenylalanine derivative $p$-azido-L-phenylalanine $(\mathrm{AzF})$, which can also be used as a crosslinker ${ }^{[47,80]}$ (see above), is suitable for infrared (IR) spectroscopy since its vibration of the azido group absorbs in a clear spectral window at $\sim 2100 \mathrm{~cm}^{-1}$, well 
separated from intrinsic protein vibrations. This has been exploited to examine fast conformational changes, that the $\mathrm{G}$ protein-coupled receptor rhodopsin undergoes after light activation and binding a cytoplasmic $\mathrm{G}$ protein, by Fourier transform infrared (FTIR) difference spectroscopy ${ }^{[99,100]}$. Another IR-labeled phenylalanine, bearing a cyano group in place of the azido moiety ( $p$-cyano-L-phenylalanine), absorbing at $\sim 2200 \mathrm{~cm}^{-1}$, was incorporated into myoglobin to probe metal ion and ligand binding ${ }^{[101]}$. Unnatural amino acids holding ${ }^{19} \mathrm{~F},{ }^{13} \mathrm{C}$ or ${ }^{15} \mathrm{~N}$ isotopes ${ }^{[70,102-104]}$, that can be detected by nuclear magnetic resonance (NMR), enable single site structural investigations of proteins, in contrast to global labeling by growth medium supplementation with ${ }^{13} \mathrm{C}$-glucose and ${ }^{15} \mathrm{~N}$-ammonium. Li et al. could perform NMR supported analysis of protein dynamics inside of cells, "in-cell NMR" (also see Ch. 5.4), since they were able to detect several site-specifically ${ }^{19} \mathrm{~F}$-labeled proteins in living E. coli ${ }^{[104]}$.

Genetically encoded UAAs were not only used to determine functionalities of proteins and their related biological processes. Liu and colleagues introduced, among others, $p$-acetyl-L-phenylalanine (pAcF) into different cis-regulatory leader-peptide elements. These small molecules served as ON or OFF switches and thereby regulated the transcription of downstream genes ${ }^{[105]}$. The same amino acid was also employeed to make the therapeutic agent, human growth hormone (hGH), more viable without perturbing its biological activity. To this end, the reactive functional group of pAcF was posttranslationally modified by PEGylation (covalent attachment of polyethylene glycol (PEG)) in a bioorthogonal "click reaction" (for "click reaction" also see next chapter and Ch. 5.1 to 5.3$)^{[48,106]}$.

\subsection{The Genetically Encoded Incorporation of FRET Pairs}

Highly sensitive fluorophores belong to a class of spectroscopic probes not mentioned in the previous chapter. They can be used to determine protein expression, activity, localization, and function ${ }^{[107]}$. GFP is one of the most widely studied and exploited fluorophores in biochemistry and cell biology ${ }^{[108]}$. However, as a fluorescent protein it needs to be correctly folded to be functional. Thus, GFP cannot be site-specifically placed at any position in a protein of interest and is therefore fused to the protein's termini. In addition, its size would perturb the structure and function of the investigated protein.

Genetically encoded (small) fluorescent UAAs, in theory, would cause minimal structural perturbation and are unlikely to impair a protein's function and localization. Unfortunately, many fluorophores are not cell-permeable or are simply too large to be a substrate for the 
aaRSs. Hence, only a small number of fluorescent UAAs have been directly incorporated into proteins ${ }^{[109-112]}$. Nevertheless, Charbon et al. were indeed able to localize the chaperonin protein GroEL under normal and stress conditions in living cells by labeling with a coumarin fluorophore containing UAA, whereas GFP fusions proved to interfere with GroEL function ${ }^{[113]}$. Summerer et al. incorporated a dansyl-UAA into the protein human superoxide dismutase to follow its denaturation in the presence of guanidinium chloride ${ }^{[110]}$.

In order to facilitate the labeling of proteins with a broader range of fluorophores, since hundreds of small organic dyes are commercially available ${ }^{[107]}$, one had to find an alternative strategy. The genetically encoded installation of an UAA containing a bioorthogonal reactive moiety provides one avenue because they allow the subsequent site-specific modification of a protein with almost any probe by bioorthogonal "click chemistry" ${ }^{[114]}$ and hence also any fluorophore that is compatible with the installed UAA.

"Click reactions" between an azide and a terminal alkyne necessitate the use of cytotoxic $\mathrm{Cu}(\mathrm{I})$ to catalyze the cycloaddition ${ }^{[115]}$, impeding in vivo applications. More recently, $\mathrm{Cu}(\mathrm{I})$ free approaches with cyclooctyne derivatives were developed, which activate the alkyne via ring strain ${ }^{[116,117]}$. The Ebright laboratory coupled fluorescent probes by Staudinger ligation to genetically encoded AzF to investigate the opening and closing of the bacterial RNA polymerase clamp ${ }^{[118]}$. Intracellular reduction is one disadvantage of genetically encoded azides. Alternative strategies employ inverse electron-demand Diels-Alder cycloaddition reactions between genetically encoded strained alkenes and alkynes and labeling reagents conjugated to tetrazines and/or azides ${ }^{[119]}$. The lysine derivatives $N \varepsilon$-5-norbornene-2yloxycarbonyl-L-lysine (NorK) and bicyclo[6.1.0]non-4-yn-9-ylmethanol-L-lysine (BCNK) have been successfully incorporated into proteins by the Chin laboratory, followed by labeling with fluorophores conjugated to tetrazines ${ }^{[120,121]}$.

The simultaneous installation of two or more fluorophores in a single polypeptide permits the investigation of conformational changes, even on a single-molecule $(\mathrm{sm})$ level, using the powerful technique Förster/Fluorescence resonance energy transfer (FRET). FRET relies on the energy transfer from a donor to an acceptor fluorophore in a distance-dependent manner and is capable of detecting distances and their changes in a nanometer scale, both in vitro and in vivo ${ }^{[122,123]}$. However, the precise and site-specific labeling of the proteins with suitable fluorophores is essential for FRET experiments but a challenging task and is often the limiting factor. Brustad et al. studied T4 lysozyme folding using FRET with sitespecific dual-labeling. One label was generated by a genetically encoded pAcF with a 
hydroxylamine-containing fluorophore and the other from a single cysteine with a maleimide conjugated fluorophore ${ }^{[124]}$. Nevertheless, this technique is restricted in its applicability to single-cysteine proteins. The incorporation of two distinct and suitable UAAs into the same protein and the subsequent bioorthogonal labeling of these provides an elegant method for the genetically encoded incorporation of FRET pairs into proteins ${ }^{[72,78]}$.

\subsection{Specific Aims}

This work is intended to apply the principles of genetic code expansion to achieve the efficient incorporation of two different unnatural amino acids into the same polypeptide, allowing us the generation of proteins with a variety of functionalities, including FRET pairs, multiple different NMR and IR probes, combinations of posttranslational modifications and bioorthogonal reactive groups for subsequent chemical modifications.

The eye of a needle for this aim is the efficiency of (multiple) UAA incorporation. Although several trials to optimize this efficiency have been performed by others in the past, none of these approaches provided a systematic study on the correlation of aaRS and tRNA expression levels including the aminoacylation status of the tRNA. Thus, only little insight is given into which of these components is the actual limiting factor. It is therefore necessary to establish assays for the detection of the abundance and activity of each component, in order to identify and optimize the constraints of the genetic code expansion system.

Once the system is optimized, we will start to focus on the production of proteins with FRET pairs to investigate their conformational states and the transitions between them. This can be studied in single-molecule FRET experiments for which the precise labeling of proteins with suitable fluorophores is a limiting factor. 


\section{Materials And Methods}

\subsection{Materials}

\subsubsection{Devices And Instruments}

AbiPrism 3100 DNA Sequencer

Accumax Pipet Help

Agarose Gel Electrophoresis Chamber

Autoclave HST 4-5-8

BioPhotometer

Biovortex V1

Bunsen Burner Fuego Basis

Cell Sorter iCyt Synergy

Centrifuge 5415R

Centrifuge Allegra 2IR

Centrifuge Avanti J-20 XPIJA-20

Centrifuge HERAEUS Pico 17

Concentrator 5301

Electroblotter (Semidry)

Electroblotter (Semidry; $20 \times 20$ cm)

Electroporator Easyject Prima

FLUOstar Omega

Gel Doc 2000

Gel Shaker Duomax 1030

Gel Shaker Rotamax 120
Applied Biosystems, Darmstadt

Accumax, INDIA

GP-Kuststofftechnik, Kassel

Zirbus, Bad Grund

Eppendorf, Hamburg

Peqlab, Erlangen

WLD-Tec, Göttingen

Sony Biotechnology, USA

Eppendorf, Hamburg

Beckman Coulter, Krefeld

Beckman Coulter, Krefeld

Thermo Scientific, Schwerte

Eppendorf, Hamburg

PeqLab, Erlangen

VWR International, Darmstadt

EquiBio, Willstätt

BMG Labtech, Ortenberg

BioRad, München

Heidolph, Schwabach

Heidolph, Schwabach 
Hamilton Syringe $50 \mu \mathrm{L}$

Hybridizer Oven HB-1000

Hypercassette $18 \times 24 \mathrm{~cm}$

Incubator Mytron WB $60 \mathrm{k}$

Labcycler

Magnetic Stirrer MR Hei-Standard

Magnetic Stirrer MR3000

Microfluidizer $110 \mathrm{~S}$

Optimax X-Ray Film Processor

pH Meter PT-15

Photometer

Pipets Research Plus $(10,100,1000 \mu \mathrm{L})$

Power Supply 300V

Power Supply EV231

Power Supply MP-250V

Rotating Mixer RM5 Assistant 348

Rotor JA-20

Rotor JLA-8.1000

Scale 770

Scanner CanoScan 5600F

Sonifier 250

Special Accuracy Scale BP410S

Tankblotter Criterion (1.3 L)

Tankblotter Mini Trans-Blot (0.45 L)
Hamilton, USA

UVP, USA

GE Healthcare, München

Mytron, Heiligenstadt

SensoQuest, Göttingen

Heidolph, Schwabach

Heidolph, Schwabach

Microfluidics, USA

Protec, Oberstenfeld

Sartorius, Göttingen

Biometra, Göttingen

Eppendorf, Hamburg

VWR International, Darmstadt

Consort, BELGIUM

Major Science, USA

Karl Hecht KG, Sondheim/Rhön

Beckman Coulter, Krefeld

Beckman Coulter, Krefeld

Kern, Balingen-Frommern

Canon Deutschland, Krefeld

Branson, USA

Sartorius, Göttingen

BioRad, München

BioRad, München 
Thermomixer comfort $1.5 \mathrm{~mL}$

Typhoon 9400 Variable Mode Imager

Unitron Incubators

UV table TFX-20.LC

Vertical Double Gel Systems

Vertical Gel Electrophoresis Chamber H10 Mini

Vortex generator VV3

X-Ray Cassette $18 \times 24$
Eppendorf, Hamburg

Amersham (GE Healthcare), München

Infors HT, Einsbach

Vilber Lourmat, Eberhardzell

PeqLab, Erlangen

GP-Kunststofftechnik, Kassel

VWR International, Darmstadt

Rego X-Ray GmbH, Augsburg

\subsubsection{Chemicals}

All chemicals were purchased from those companies listed below, unless stated otherwise, and fulfilled the purity grade "pro analysis".

AppliChem, Darmstadt

BioRad, München

Merck, Darmstadt

Roth ,Karlsruhe

Sigma-Aldrich, Steinheim

VWR International, Darmstadt

\subsubsection{Consumables And Other Materials}

96-Well Black Microplates

Amersham ECL Plus WB Detection Reagent

Amersham ECL Prime WB Detection Reagent

Amersham ECL Select WB Detection Reagent
VWR International, Darmstadt

GE Healthcare, München

GE Healthcare, München

GE Healthcare, München 
Amersham Hyperfilm ECL

Amicon Ultra-15 Centrifugal Filter Units

(NMWL 3 kDa, 10 kDa, 30 kDa, 50 kDa)

CDP-Star ECL Substrate

Centrifugal Ultrafiltration Devices

(MWCO 3k, 10k)

Coli Rollers Plating Beads

Deep Well Plates, $2 \mathrm{~mL}$ - Sterile

Electroporation Cuvettes, 2 mm gap

Eppendorf Tubes $(1.5 \mathrm{~mL}, 2.0 \mathrm{~mL}$ )

Falcon Tubes ( $15 \mathrm{~mL}, 50 \mathrm{~mL}$ )

Gas Permeable Adhesive Seals

GSH Sepharose Beads

HisLink Protein Purification Resin

Hybond-N+ Transfer Membrane, Nylon

Immobilon-P Transfer Membrane, PVDF

Instant Blue

PCR Soft Tubes $(0.2 \mathrm{~mL})$

peqGOLD Universal Agarose

Petri Dishes $145 \times 20 \mathrm{~mm}$

Petri Dishes $92 \times 16 \mathrm{~mm}$

Pipet Tips

Plate 96 DW Square U

Protran Nitrocellulose Transfer Membrane

Slide-A-Lyzer ${ }^{\circledast}$ Mini Dialysis Units
GE Healthcare, München

Merck, Darmstadt

Roche, Mannheim

Sartorius, Göttingen

Novagen, USA

Axygen Scientific, USA

Peqlab, Erlangen

Eppendorf, Hamburg

Sarstedt, Nümbrecht

Thermo Scientific, Schwerte

GE Healthcare, München

Promega, USA

GE Healthcare, München

Merck, Darmstadt

Biozol, Eching

Biozym, Austria

Peqlab, Erlangen

Greiner Bio One, Solingen

Sarstedt, Nümbrecht

Sarstedt, Nümbrecht

VWR International, Darmstadt

Whatman, Dassel

Thermo Scientific, Schwerte 
Spectra/Por Closures, $55 \mathrm{~mm}$

Spectra/Por Dialysis Membrane, $50 \mathrm{~mm}$

Syringe Filters ( 0.2 and $0.45 \mu \mathrm{m})$

UV Cuvettes (UVette)

Whatman filter paper
Spectrum Laboratories, Netherlands

Spectrum Laboratories, Netherlands

Sartorius, Göttingen

Eppendorf, Hamburg

Whatman, Dassel

\subsubsection{DNA, Protein And RNA Size Standards}

GeneRuler $^{\mathrm{TM}} 1 \mathrm{~kb}$ DNA Ladder

PageRuler Prestained Protein Ladder

Unstained Protein Molecular Weight Marker

Low Molecular Weight Marker 10-100 nt

Low Range ssRNA Ladder

Oligonucleotide Ladder (20, 35, 45, 58, 70 nt)
Thermo Scientific, Schwerte

Thermo Scientific, Schwerte

Thermo Scientific, Schwerte

USB $^{\circledR}$ Products/Affymetrix, USA

NEB, Frankfurt

Self-made from Sigma-Aldrich primers

\subsubsection{Antibiotics}

Table 3.1: Overview of antibiotics used in growth media/agar plates

\begin{tabular}{lcl} 
Antibiotic & $\begin{array}{c}\text { Work Concentration } \\
{[\mu \mathrm{g} / \mathrm{mL}]}\end{array}$ & Company \\
\hline Ampicillin (Amp) & 100 & AppliChem, Darmstadt \\
Chloramphenicol $(\mathrm{Cm})$ & 50 & AppliChem, Darmstadt \\
Kanamycin (Kan) & 50 & AppliChem, Darmstadt \\
Spectinomycin (Sm) & 75 & Sigma-Aldrich, Steinheim \\
Tetracycline (Tet) & 25 & AppliChem, Darmstadt
\end{tabular}




\subsubsection{Culture Media And Agar Plates}

For agar plates the corresponding medium was supplemented with $1.5 \%(\mathrm{w} / \mathrm{v})$ agar-agar. M9 salts, LB and 2 YT medium were sterilized by autoclaving at $121{ }^{\circ} \mathrm{C}$ for $20 \mathrm{~min}$. Antibiotics and other supplements were added after cooling to at least $55^{\circ} \mathrm{C}$.

Table 3.2: Overview of growth media used for culturing of $E$. coli

\begin{tabular}{|c|c|c|}
\hline LB Medium & 2YT Medium & M9 Minimal Medium \\
\hline $10 \mathrm{~g}$ tryptone & $16 \mathrm{~g}$ tryptone & $200 \mathrm{~mL}$ M9 salts $(5 x)$ \\
\hline $5 \mathrm{~g}$ yeast extract & $10 \mathrm{~g}$ yeast extract & $2 \mathrm{~mL} \mathrm{MgSO}_{4}(1 \mathrm{M})$ \\
\hline $5 \mathrm{~g} \mathrm{NaCl}$ & $5 \mathrm{~g} \mathrm{NaCl}$ & $20 \mathrm{~mL}$ glucose $(20 \% ; \mathrm{w} / \mathrm{v})$ \\
\hline \multirow[t]{7}{*}{ 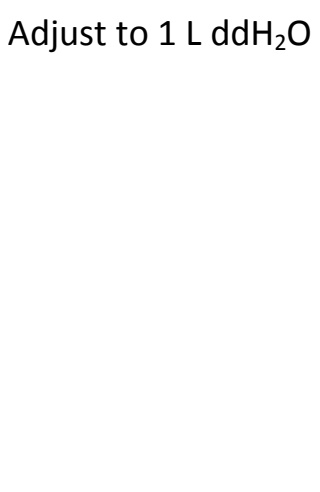 } & Adjust to $1 \mathrm{LddH}_{2} \mathrm{O}$ & $0.1 \mathrm{~mL} \mathrm{CaCl} 2(1 \mathrm{M})$ \\
\hline & & Adjust to $1 \mathrm{~L} \mathrm{ddH_{2 } O}$ \\
\hline & & M9 salts $(5 x)$ \\
\hline & & $250 \mathrm{mM} \mathrm{Na}_{2} \mathrm{HPO}_{4}$ \\
\hline & & $100 \mathrm{mM} \mathrm{KH}_{2} \mathrm{PO}_{4}$ \\
\hline & & $50 \mathrm{mM} \mathrm{NaCl}$ \\
\hline & & $100 \mathrm{mM} \mathrm{NH}_{4} \mathrm{Cl}$ \\
\hline
\end{tabular}

\subsubsection{Unnatural Amino Acids}

Unnatural amino acids (UAAs) were dissolved with $\mathrm{NaOH}$ in $\mathrm{ddH}_{2} \mathrm{O}$ (see Table 3.3) just before the addition to the medium.

Table 3.3: Overview of unnatural amino acids used for genetic code expansion

\begin{tabular}{|c|c|c|c|}
\hline Amino acid & $\begin{array}{c}\text { Stock Solution } \\
{[\mathrm{M}]}\end{array}$ & $\begin{array}{c}\text { Work Concentration } \\
{[\mathrm{mM}]}\end{array}$ & Company \\
\hline $\begin{array}{l}\text { Boc-L-lysine } \\
\text { (BocK) }\end{array}$ & $\begin{array}{c}0.5 \\
\text { (in } 1 \mathrm{M} \mathrm{NaOH} \text { ) }\end{array}$ & 1 & Bachem, Bubendorf $(\mathrm{CH})$ \\
\hline $\begin{array}{l}\text { 4-azido-L- } \\
\text { phenylalanine (AzF) }\end{array}$ & $\begin{array}{c}0.5 \\
\text { (in } 0.5 \mathrm{M} \mathrm{NaOH} \text { ) }\end{array}$ & $1-5$ & ChemImpex, USA \\
\hline $\begin{array}{l}\text { Norbornene-L-lysine } \\
\text { (NorK) }\end{array}$ & $\begin{array}{c}0.1 \\
\text { (in } 0.2 \mathrm{M} \mathrm{NaOH} \text { ) }\end{array}$ & 2 & AG Hell, Göttingen \\
\hline $\begin{array}{l}\text { Bicyclononynes-L- } \\
\text { lysine (BCNK) }\end{array}$ & $\begin{array}{c}0.1 \\
\text { (in } 0.2 \mathrm{M} \mathrm{NaOH} \text { ) }\end{array}$ & 2 & SynAffix, Nijmegen (NL) \\
\hline
\end{tabular}




\subsubsection{Enzymes}

Enzymes were used as recommended by the company's protocol. The following table gives an overview about the enzymes used in this study.

Table 3.4: Overview of enzymes used

Enyzme

Company

T4 DNA ligase

Thermo Scientific, Schwerte

Shrimp Alkaline Phosphatase (SAP)

Taq DNA Polymerase

Phusion DNA Polymerase

Restriction enzymes: BamHI, Dpnl, HindIII, Kpnl,

Mfel, Ncol, Ndel, Notl, Pstl, Sacl, Sall, Stul, Xhol

Proteinase $\mathrm{K}$

Alkaline Phosphatase, Calf Intestinal (CIP)

NEB, Frankfurt

Restriction enzymes: Agel, BamHI, Bsal, Dpnl,

Ndel, Nhel, Sall, Stul

Expand High Fidelity Polymerase Mix

Roche, Mannheim

Pfu Turbo DNA Polymerase

Agilent, Böblingen

\subsubsection{Cell Lines}

Table 3.5: Overview of cell lines used

\begin{tabular}{|c|c|c|}
\hline Cell line & Genotype & Company \\
\hline \multirow[t]{2}{*}{ E. coli $\mathrm{DH} 10 \mathrm{~B}$} & $\mathrm{~F}^{-} m c r \mathrm{~A} \Delta(m r r-h s d \mathrm{RMS}-m c r \mathrm{BC})$ & Invitrogen, \\
\hline & $\begin{array}{l}\text { Ф80/acZ } 1 \mathrm{M} 15 \Delta / a c X 74 \text { recA1 endA1 araD139A } \\
\text { (ara,leu)7697 ga/U ga/K } \lambda^{-} \text {rpsL nupG }\end{array}$ & Darmstadt \\
\hline \multirow[t]{3}{*}{ E. coli BL21 (DE3) } & fhuA2 [lon] ompT gal ( $\lambda$ DE3) [dcm] $\Delta h s d S$ & NEB, Frankfurt \\
\hline & $\lambda D E 3=\lambda$ sBamHIo $\triangle E c o R I-B$ & \\
\hline & int::(lacl::PlacUV5::T7 gene1) i21 $\Delta$ nin5 & \\
\hline E. coli SHuffle & fhuA2 lacZ::T7 gene1 [lon] ompT ahpC & NEB, Frankfurt \\
\hline \multirow[t]{4}{*}{ T7 Express } & gal $\lambda$ att::pNEB3-r1-cDsbC (SpecR, $\left.\mid a c l^{q}\right) \Delta \operatorname{trx} B$ & \\
\hline & sulA11 R(mcr-73::miniTn10--Tet $\left.{ }^{\mathrm{S}}\right) 2[\mathrm{dcm}]$ & \\
\hline & $R\left(z g b-210:: \operatorname{Tn} 10--\right.$ Tet $\left.^{\mathrm{S}}\right)$ end $A 1 \Delta$ gor $\Delta(\operatorname{mcrC}-$ & \\
\hline & $m r r) 114:: I S 10$ & \\
\hline
\end{tabular}




\subsubsection{Buffers And Solutions}

All buffers were prepared with $\mathrm{dd}_{2} \mathrm{O}$.

Table 3.6: Overview of commonly used buffers $\&$ solutions

\section{Buffer/Solution}

PIC (1x)
Ingredients

$75 \mu \mathrm{M}$ pefabloc SC

$150 \mathrm{nM}$ leupeptin

37.5 $\mu \mathrm{M}$ O-phenanthroline

$500 \mathrm{nM}$ pepstatin A

Sterilize using syringe filters $(0.2 \mu \mathrm{m})$

\begin{tabular}{|c|c|}
\hline PBS (1x) & $137 \mathrm{mM} \mathrm{NaCl}$ \\
\hline & $2.7 \mathrm{mM} \mathrm{KCl}$ \\
\hline & $10 \mathrm{mM} \mathrm{Na}_{2} \mathrm{HPO}_{4}$ \\
\hline & $1.8 \mathrm{mM} \mathrm{KH}_{2} \mathrm{PO}_{4}$ \\
\hline & $\mathrm{pH} 7.4$ \\
\hline TBS (1x) & $50 \mathrm{mM}$ TRIS-HCl, pH 7.5 \\
\hline & $150 \mathrm{mM} \mathrm{NaCl}$ \\
\hline $\mathrm{CaCl}_{2}$ Solution & $60 \mathrm{mM} \mathrm{CaCl}_{2}$ \\
\hline & 10 mM Pipes-KOH, pH 7.0 \\
\hline & $15 \%$ glycerol (v/v) \\
\hline & Autoclave \\
\hline & Store at $4{ }^{\circ} \mathrm{C}$ \\
\hline TBE (1x) & $89 \mathrm{mM}$ TRIS base \\
\hline & $89 \mathrm{mM}$ boric acid \\
\hline & $2 \mathrm{mM}$ EDTA- $\mathrm{Na}_{2}$ \\
\hline DNA Loading Buffer $(1 x)$ & $3 \%$ glycerol (v/v) \\
\hline & 1 mM TRIS base, pH 7.5 \\
\hline & $1 \mathrm{mM}$ EDTA-Na ${ }_{2}$ \\
\hline & Bromphenol blue \\
\hline & Xylene cyanol \\
\hline SDS Running Buffer (1x) & $25 \mathrm{mM}$ TRIS base \\
\hline & 192 mM glycine \\
\hline & $0,1 \%$ SDS (w/v) \\
\hline
\end{tabular}




\begin{tabular}{|c|c|}
\hline \multirow[t]{5}{*}{ SDS Sample Buffer (1x) } & $2.5 \%$ glycerol $(\mathrm{v} / \mathrm{v})$ \\
\hline & $12.5 \mathrm{mM}$ TRIS-HCl, pH 6.8 \\
\hline & $25 \mathrm{mM}$ DTT \\
\hline & $0.5 \% \operatorname{SDS}(\mathrm{w} / \mathrm{v})$ \\
\hline & $0.025 \%$ bromphenol blue $(\mathrm{w} / \mathrm{v})$ \\
\hline \multirow[t]{2}{*}{ WB Transfer Buffer (1x) } & $1 \times$ SDS running buffer \\
\hline & $15-20 \% \mathrm{MeOH}(\mathrm{v} / \mathrm{v})$ \\
\hline \multirow[t]{2}{*}{ PonceauS Solution (0.5\%) } & $0.5 \%$ PonceauS (w/v) \\
\hline & $5 \%$ TCA $(w / v)$ \\
\hline \multirow[t]{2}{*}{ TE buffer $(1 x)$} & $10 \mathrm{mM}$ TRIS, $\mathrm{pH} 8.0$ \\
\hline & $1 \mathrm{mM}$ EDTA- $\mathrm{Na}_{2}$ \\
\hline \multirow[t]{4}{*}{ Acid Urea Sample Buffer } & $0.1 \mathrm{M}$ sodium acetate, $\mathrm{pH} 5.0$ \\
\hline & $8 \mathrm{M}$ urea \\
\hline & $0.05 \%$ bromphenol blue $(\mathrm{w} / \mathrm{v})$ \\
\hline & $0.05 \%$ xylene cyanol $(\mathrm{w} / \mathrm{v})$ \\
\hline Acid Urea Running Buffer (1x) & $0.1 \mathrm{M}$ sodium acetate, $\mathrm{pH} 5.0$ \\
\hline \multirow[t]{2}{*}{ NB Transfer Buffer (1x) } & $40 \mathrm{mM}$ TRIS-HCl, $\mathrm{pH} 8.0$ \\
\hline & $2 \mathrm{mM}$ EDTA-Na ${ }_{2}$ \\
\hline \multirow[t]{3}{*}{ SSC Buffer (1x) } & $150 \mathrm{mM} \mathrm{NaCl}$ \\
\hline & $15 \mathrm{mM}$ trisodium citrate \\
\hline & $\mathrm{pH} 7.0$ \\
\hline \multirow[t]{3}{*}{ Prehybridization Solution (1x) } & $6 \times \mathrm{SSC}$ \\
\hline & 10x Denhardt's solution (Invitrogen) \\
\hline & $0.5 \% \operatorname{SDS}(\mathrm{w} / \mathrm{v})$ \\
\hline \multirow[t]{4}{*}{ Hybridization Solution (1x) } & $6 \times \mathrm{SSC}$ \\
\hline & $0.1 \% \operatorname{SDS}(\mathrm{w} / \mathrm{v})$ \\
\hline & tRNA probe 1:3000 (v/v) \\
\hline & Sterilize using syringe filters $(0.2 \mu \mathrm{m})$ \\
\hline \multirow[t]{3}{*}{ Buffer $1(1 \times)$} & $100 \mathrm{mM}$ maleic acid \\
\hline & $150 \mathrm{mM} \mathrm{NaCl}$ \\
\hline & pH 7.5 (with $\mathrm{NaOH}$ pellets) \\
\hline \multirow[t]{3}{*}{ Blocking Reagent (10\%) } & $10 \%$ blocking reagent ((w/v); Roche) \\
\hline & $1 \times$ buffer 1 \\
\hline & Autoclave and store at $4{ }^{\circ} \mathrm{C}$ \\
\hline
\end{tabular}




\begin{tabular}{ll}
\hline Buffer 2 (1x) & $10 \%$ blocking reagent, 10\% (v/v) \\
& $1 \times$ buffer 1 \\
\hline Buffer 3 (1x) & $100 \mathrm{mM}$ TRIS base, $\mathrm{pH} 9.5$ \\
& $100 \mathrm{mM} \mathrm{NaCl}$
\end{tabular}

\subsubsection{DNA Kit Systems}

Kits were used according to manufacturer's protocol, except for XP2 binding buffer from peqGOLD Gel Extraction Kit and CP binding buffer from Cycle Pure Kit. XP2 was used in a $\mathrm{w}($ gel)/v(buffer) ratio of $1: 3$ instead of $1: 1$ and $C P$ buffer was used in a $v(P C R) / v($ buffer) ratio of $1: 5$ instead of $1: 1$.
peqGOLD Cycle-Pure Kit (S-Line)
Peqlab, Erlangen
peqGOLD Gel Extraction Kit (S-Line)
Peqlab, Erlangen
peqGOLD Plasmid Miniprep Kit I\& II
Peqlab, Erlangen
QIAGEN Plasmid Mini Kit
QIAGEN, Hilden
QIAquick Gel Extraction Kit
QIAGEN, Hilden
QIAquick PCR Purification Kit
QIAGEN, Hilden

\subsubsection{Fluorescent Dyes}

Table 3.7: Overview of fluorescent dyes used for protein labeling.

All dyes were prepared as stock solutions with $10 \mathrm{mg} / \mathrm{mL}$ in DMSO. Abs. Max. = Absorption Maximum; Em. Max = Emission Maxium; Abb. = Abberior

\begin{tabular}{lccll} 
Dye & $\begin{array}{c}\text { Abs. Max. } \\
\text { [nm] }\end{array}$ & $\begin{array}{c}\text { Em. Max. } \\
{[\mathbf{n m}]}\end{array}$ & Conjugate & Company \\
\hline Atto565 & 563 & 592 & Maleimide & ATTO-TEC, Siegen \\
Abb. Star635 & 634 & 654 & Tetrazine & AG Hell, Göttingen ${ }^{[125]}$ \\
Abb. Star635P & 635 & 655 & Tetrazine & AG Hell, Göttingen ${ }^{[125]}$ \\
KK9046 & 632 & 654 & Tetrazine & AG Hell, Göttingen ${ }^{[125]}$ \\
KK114 & 637 & 660 & Tetrazine & AG Hell, Göttingen ${ }^{[125]}$
\end{tabular}




\subsubsection{Antibodies}

Table 3.8: Overview of antibodies used for immuno blotting (western \& northern blots).

His = histidine; GFP = green fluorescent protein; GST = glutathione $S$-transferase; $\mathrm{DIG}=$ digoxigenin; $\mathrm{Myc}=$ myelocytomatosis $\quad \mathrm{MBP}=$ maltose binding protein; $\mathrm{AP}=$ alkaline phosphatase $; \mathrm{HRP}=$ horseradish peroxidase

\begin{tabular}{|c|c|c|c|c|c|c|}
\hline & Antigen & Host & Conjugate & Diluent (w/v) & $\begin{array}{l}\text { Product } \\
\text { code }\end{array}$ & Company \\
\hline \multirow{6}{*}{ 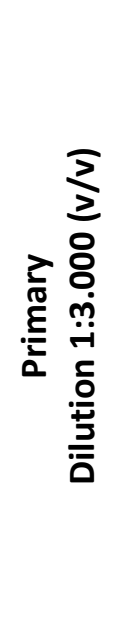 } & His & mouse & - & $3 \%$ BSA-PBS & 27-4710-01 & $\begin{array}{l}\text { GE Healthcare, } \\
\text { München }\end{array}$ \\
\hline & GFP & mouse & - & $3 \%$ BSA-PBS & sc-9996 & $\begin{array}{l}\text { Santa Cruz, } \\
\text { Heidelberg }\end{array}$ \\
\hline & GST & goat & - & $3 \%$ BSA-PBS & 27-4577-01 & $\begin{array}{l}\text { GE Healthcare, } \\
\text { München }\end{array}$ \\
\hline & DIG & sheep & AP & $\begin{array}{l}1: 10.000 \text { in } \\
1 \times \text { Buffer } 2\end{array}$ & 11093274910 & $\begin{array}{l}\text { Roche, } \\
\text { Mannheim }\end{array}$ \\
\hline & Myc & mouse & - & 5\% Milk-TBS & sc-40 & $\begin{array}{l}\text { Santa Cruz, } \\
\text { Heidelberg }\end{array}$ \\
\hline & MBP & rabbit & - & $3 \%$ BSA-PBS & \#E8030S & NEB, Frankfurt \\
\hline \multirow{3}{*}{ 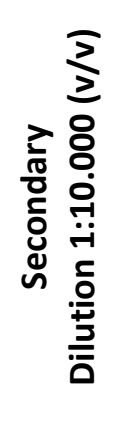 } & mouse & goat & HRP & $\begin{array}{l}\text { 5\% Milk-PBS } \\
\text { (TBS for Myc) }\end{array}$ & $\begin{array}{l}\text { ab6789 / } \\
\text { A8924 }\end{array}$ & $\begin{array}{l}\text { Abcam, UK / } \\
\text { Sigma-Aldrich, } \\
\text { Steinheim }\end{array}$ \\
\hline & rabbit & goat & HRP & 5\% Milk-PBS & $\begin{array}{l}\text { ab6721 / } \\
\text { R5506 }\end{array}$ & $\begin{array}{l}\text { Abcam, UK / } \\
\text { Sigma-Aldrich, } \\
\text { Steinheim }\end{array}$ \\
\hline & goat & rabbit & $A P$ & 5\% Milk-PBS & A4187 & $\begin{array}{l}\text { Sigma-Aldrich, } \\
\text { Steinheim }\end{array}$ \\
\hline
\end{tabular}




\subsection{Methods}

\subsubsection{Microbiological Methods}

\subsubsection{Chemical Competent Cells}

Chemical competent cells were prepared from overnight starting cultures by expanding the cells to an $\mathrm{OD}_{600}$ of 0.1 , in the desired volume needed. After incubation at $37^{\circ} \mathrm{C}$ cells were harvested at an $\mathrm{OD}_{600}$ of $0.6-0.8$ by splitting them into $50 \mathrm{~mL}$ aliquots followed by centrifugation $\left(4^{\circ} \mathrm{C}, 15 \mathrm{~min}, 4,147 \times \mathrm{g}\right)$. All following steps were performed on ice. Each pellet was washed with $10 \mathrm{~mL} \mathrm{CaCl}$ solution (Table 3.6) and centrifuged as before. Afterwards, cells were washed again with $10 \mathrm{~mL} \mathrm{CaCl}$ solution but incubated at $4{ }^{\circ} \mathrm{C}$ for $1 \mathrm{~h}$ before subsequent final centrifugation. Pellets were resuspended in $2 \mathrm{~mL} \mathrm{CaCl}$ solution and frozen in liquid nitrogen as $200 \mu \mathrm{L}$ aliquots or used directly for transformations.

\subsubsection{Electro Competent Cells}

For the production of electro competent cells a main culture was grown, split and centrifuged as described in chapter 3.2.1.1. Remaining salts were removed by washing twice with $10 \mathrm{~mL} 15 \%$ glycerol $/ \mathrm{H}_{2} \mathrm{O}(\mathrm{v} / \mathrm{v})$ at $4{ }^{\circ} \mathrm{C}$. After the final centrifugation pellets were resuspended in reflow (remaining supernatant), pooled and directly used for transformations ${ }^{[126]}$.

\subsubsection{Transformation of Chemical Competent Cells}

Chemically competent $E$. coli cells were transformed with plasmids using the heat shock method. $50 \mu \mathrm{L}$ of chemical competent cells (Ch. 3.2.1.1) were mixed with 150-500 ng DNA and incubated on ice for $10 \mathrm{~min}$. After heat shocking for $2 \mathrm{~min}$ at $42^{\circ} \mathrm{C}$, the cells were put back on ice for $3 \mathrm{~min}$. For the recovery, $1 \mathrm{~mL}$ LB medium (Table 3.2) was added, followed by incubation $\left(37^{\circ} \mathrm{C}, 750 \mathrm{rpm}\right.$ ) for $1 \mathrm{~h}$. Transformants were plated on agar media containing the appropriate antibiotics (Table 3.1) or used for the inoculation of an overnight culture.

The heat shock method was suitable for the transformation with up to two plasmids at once. If cells needed to be transformed with more plasmids, a two-step variant via the preparation of electro competent cells (Ch. 3.2.1.2) was used. 


\subsubsection{Transformation of Electro Competent Cells}

Electro competent cells were transformed with up to $1 \mu \mathrm{g}$ of plasmid DNA, typically prepared by ethanol precipitation (Ch. 3.2.3.7). A maximum of $10 \mu \mathrm{L}$ of DNA was added to $100 \mu \mathrm{L}$ electro competent cells (Ch. 3.2.1.2) and then transferred into precooled electroporation cuvettes. Electro shock was performed at $2.5 \mathrm{kV}$. For the recovery, $1 \mathrm{~mL}$ 2YT medium (Table 3.2) was added and the suspension transferred to an Eppendorf tube, followed by incubation $\left(37^{\circ} \mathrm{C}, 600 \mathrm{rpm}\right.$ ) for $1.5 \mathrm{~h}$. Transformants were plated on agar plates containing appropriate antibiotics (Table 3.1) or used for the inoculation of an overnight culture. In some cases a dilution series was plated to analyze the diversity/efficiency of the transformation $^{[126]}$.

\subsubsection{Protein Biochemical Methods}

\subsubsection{Recombinant Protein Expression}

Recombinant protein expressions were prepared from cells transformed with the appropriate plasmids. Depending on the type of induction of the protein expression, E. coli DH10B or BL21 (DE3) (for T7 driven promoters, Table 3.5) were used.

Overnight precultures were used to inoculate main cultures $(20 \mathrm{~mL}$ to several liters $L B$ or $2 Y T$ medium containing the required antibiotics, Table 3.1) to an $\mathrm{OD}_{600}$ of 0.1. Cells were incubated at $37^{\circ} \mathrm{C}, 200 \mathrm{rpm}$, to an $\mathrm{OD}_{600}$ of 0.8 -1.0. Protein expression was induced by supplementing the media with either, $0.2 \%$ arabinose $(\mathrm{w} / \mathrm{v}$ ) or $1 \mathrm{mM}$ IPTG (final concentrations). In some cases no addition of an inducer was necessary since the promoter was constitutively active. For the incorporation of UAAs into proteins of interest, the media was supplemented with the appropriate probe by first dissolving (see Table 3.3) and then added to the main culture. This was performed at inoculation when constitutive promoters were being used or in combination with the inducers for inducible promoters.

Cells were typically harvested at 4-6 $\mathrm{h}$ after induction by centrifugation, washed with PBS and stored at $-20^{\circ} \mathrm{C}$ or directly used for cell lysis (Ch. 3.2.2.2). 


\subsubsection{Cell Lysis}

Several methods to lyse bacterial cell pellets were used. For whole cell lysate analysis, small pellets from up to $2 \mathrm{~mL}$ medium were boiled in $1 \times$ SDS sample buffer (Table 3.6) for $10 \mathrm{~min}$ at $95^{\circ} \mathrm{C}$. Samples were subsequently centrifuged $(16,100 \times g, \mathrm{RT}, 5 \mathrm{~min})$ and directly used for SDS PAGE (Ch. 3.2.2.3). For protein isolation, larger cell pellets (from $50 \mathrm{~mL}$ to $500 \mathrm{~mL}$ medium) were resuspended in 20-50 mL lysis buffer, which was generally supplemented with protease inhibitors (1× PIC and 1mM PMSF in DMSO; Table 3.6), $1 \mathrm{mg} / \mathrm{mL}$ lysozyme, $0.5 \mathrm{mg} / \mathrm{mL}$ DNase I and $1 \mathrm{mM}$ DTT (see Ch. 3.2.2.6 to 3.2.2.9 for details). Cells were then incubated on ice for 20-30 min. Disruption of the cells was carried out on ice by either ultrasonic sound, using a sonifier, or by pressure ( $80 \mathrm{psi})$ using a pneumatic cell disintegrator (fluidizer). The lysate was clarified by centrifugation $\left(20,000 \times g, 4{ }^{\circ} \mathrm{C}, 30 \mathrm{~min}\right)$. Afterwards, the supernatant could be employed for protein purification.

\subsubsection{Discontinuous Sodium Dodecyl Sulfate Polyacrylamide Gel Electrophoresis}

Table 3.9: Composition of polyacrylamide gels for SDS PAGE

\begin{tabular}{lll}
$\begin{array}{l}\text { Resolving Gel } \\
\mathbf{1 2} \%\end{array}$ & $\mathbf{1 5 \%}$ & $\begin{array}{l}\text { Stacking Gel } \\
\mathbf{4} \%\end{array}$ \\
\hline $\mathbf{3 7 5} \mathrm{mM}$ TRIS-HCl, pH 8.8 & $375 \mathrm{mM}$ TRIS-HCl, pH 8.8 & $125 \mathrm{mM}$ TRIS-HCl, pH 6.8 \\
$0.1 \%$ SDS $(\mathrm{w} / \mathrm{v})$ & $0.1 \%$ SDS $(\mathrm{w} / \mathrm{v})$ & $0.1 \% \mathrm{SDS}(\mathrm{w} / \mathrm{v})$ \\
$12 \%$ acrylamide/ & $15 \%$ acrylamide/ & $4 \%$ acrylamide/ \\
$0.32 \%$ bisacrylamide & $0.4 \%$ bisacrylamide & $0.11 \%$ bisacrylamide \\
$0.1 \%$ APS $(\mathrm{w} / \mathrm{v})$ & $0.1 \%$ APS $(\mathrm{w} / \mathrm{v})$ & $0.05 \%$ APS $(\mathrm{w} / \mathrm{v})$ \\
$0.04 \%$ TEMED $(\mathrm{v} / \mathrm{v})$ & $0.04 \%$ TEMED $(\mathrm{v} / \mathrm{v})$ & $0.1 \%$ TEMED $(\mathrm{v} / \mathrm{v})$
\end{tabular}

Discontinuous sodium dodecyl sulfate polyacrylamide gel electrophoresis (SDS PAGE) as described by Laemmli ${ }^{[127]}$ was performed to analyze the size and purity of proteins. Thereby, the strong anionic detergent SDS causes the denaturation of proteins and confers a negative charge to them, simultaneously. The discontinuity between stacking and resolving gel relies on different pore sizes and $\mathrm{pH}$ values, as well. The $\mathrm{pH}$ gradient is responsible for the stacking of the proteins at the border to the resolving gel. Whereas the stacking gels always have the same concentration of acrylamide, those of the resolving gels were varied depending on the expected protein size. During this study 12 and $15 \%$ gels were used. Protein ladders (Ch. 3.1.4) helped to estimate the molecular weights of the 
separated proteins. Before loading a gel, protein solutions were mixed with $4 \times$ sample buffer heated to $95^{\circ} \mathrm{C}$ for $10 \mathrm{~min}$ followed by a short centrifugation. Small cell pellets were prepared as described in Ch. 3.2.2.2. Electrophoresis was performed at $200 \mathrm{~V}$ for 60 120 min in $1 \times$ SDS running buffer until the bromphenol blue dye traveled the length of the gel.

After electrophoresis was performed, separated proteins were either visualized by Coomassie Brilliant Blue staining (with Instant Blue, according to manufacturer's manual) or transferred to nitrocellulose, or PVDF, membrane by western blotting (Ch. 3.2.2.4).

\subsubsection{Western Blot}

Immunoblotting was performed to verify the expression of proteins by the direct transfer of proteins from SDS-PAGE (Ch. 3.2.2.3) onto membrane, followed by incubation with specific antibodies against the protein itself, or a protein tag, e.g., $\mathrm{His}_{6}$-tag.

Semidry blots of SDS gels were performed with nitrocellulose membranes, sandwiched between three layers of Whatman filter papers on each side, which were soaked in $1 \times$ WB transfer buffer. This stack was fixed in a semidry blotter and the transfer was carried out with an electric currency of $250 \mathrm{~mA}$ constant for $45 \mathrm{~min}, \mathrm{RT}$.

Wet blots were performed with PVDF membrane that was first activated with $\mathrm{MeOH}$ and then washed with water followed by soaking in $1 \times$ WB transfer buffer. The membrane, SDS gel and Whatman filter papers were assembled according to the instruction manual of the blotter. The transfer was applied at $100 \mathrm{~V}$ constant for $30-90 \mathrm{~min}$, at $4{ }^{\circ} \mathrm{C}$.

The success of the transfer was determined by Coomassie staining of the gel and PonceauS staining of the membrane. PVDF membrane was first washed with water and $\mathrm{MeOH}$ before staining with PonceauS. PonceauS stain was removed from the membranes by washing in water, followed by incubation in TBS or PBS (determined by primary antibody buffer composition). Blocking of the membrane was performed for at least $10 \mathrm{~min}$ in buffer conditions similar to the primary antibody of interest (minus the antibody).

Primary antibodies (Table 3.8) were incubated on the membrane for $2 \mathrm{~h}$ with shaking, at RT or overnight at $4{ }^{\circ} \mathrm{C}$. The membranes were then washed three times, for $10 \mathrm{~min}$, in the appropriate buffer (TBS or PBS) plus $0.1 \%$ Tween20 (v/v). Secondary antibody was applied and allowed to incubate with shaking, for $1 \mathrm{~h}$, at RT. The membrane was then washed as 
previously described. Remaining detergent was removed by additional washing with water, followed by PBS or TBS.

Chemiluminescence detection of protein was performed dependent upon the enzyme conjugated to the secondary antibody (Table 3.8). The ECL substrates used were, CDP-Star for AP-conjugates and Amersham ECL WB reagents for HRP-conjugates. The substrates were incubated on the membranes for 5 min prior to detection. In an X-ray cassette, emitted light was captured on ECL films for several seconds, to minutes, until the desired band intensities were achieved. The films were developed in an automatic X-ray film processor.

\subsubsection{Measuring Protein Concentration}

Protein concentrations were measured using the Bradford assay developed by Marion Bradford $^{[128]}$. The reactive reagent is based on Coomassie Brilliant Blue G-250, which turns from its unbound red form to blue upon protein binding. This causes a shift of the absorption spectrum maximum of the dye to $595 \mathrm{~nm}$ in a proportional manner. The linear range of the assay depends on the protein and fluctuates between 0 and $2 \mathrm{mg} / \mathrm{mL}$. Therefore, protein samples often needed to be diluted.

In general, $10 \mu \mathrm{L}$ of protein sample was mixed with $500 \mu \mathrm{L}$ Bradford reagent, incubated at RT for $5 \mathrm{~min}$ and then the absorbance was measured at $595 \mathrm{~nm}$. Water or buffer without protein served as a reference. The amount of protein was estimated by means of a standard curve made with dissolved BSA.

\subsubsection{Small Scale GST-MBP Expression And Purification}

Overnight cultures of E. coli DH10B containing the appropriate plasmid combinations (see Figure 4.37) were used to inoculate $50 \mathrm{~mL}$ main cultures to $\mathrm{OD}_{600}$ of 0.1 . The UAA BocK was supplemented to the main cultures at $\mathrm{OD}_{600}$ of 0.6 to 0.8 to a final concentration of $1 \mathrm{mM}$ and the lac promoter dependent expression of PyIS was induced by a final concentration of $1 \mathrm{mM}$ IPTG. Cells with three plasmids were pelleted $\left(4^{\circ} \mathrm{C}, 15 \mathrm{~min}, 4,147 \times \mathrm{g}\right) 280 \mathrm{~min}$ after UAA addition and with four plasmids after $330 \mathrm{~min}$. Pellets were washed with $1 \times$ PBS and cell counts were normalized by $\mathrm{OD}_{600}$, followed by centrifugation as before. GST-MBP fusion proteins were purified as described by Neumann ${ }^{[78]}$. Cells were resuspended and lysed in 
$1 \mathrm{~mL}$ lysis buffer (1× PBS supplemented with $1 \times$ BugBuster (Novagen), 1× PIC, $1 \mathrm{mM} \mathrm{PMSF}$, lysozyme, $0.5 \mathrm{mg} / \mathrm{mL}$ DNasel) for $2 \mathrm{~h}$ on ice. Undigested DNA was sheared by ultrasonic sound using a sonifier and the lysate was subsequently clarified by centrifugation $\left(4^{\circ} \mathrm{C}\right.$, $30 \mathrm{~min}, 16,100 \times \mathrm{g}$ ). Batch affinity purification of GST containing proteins was performed using $50 \mu \mathrm{L}$ of glutathione sepharose beads with shaking $\left(1 \mathrm{~h}, 4^{\circ} \mathrm{C}\right)$. Beads were washed 3 times with $1 \mathrm{~mL} 1 \times \mathrm{PBS}$, before elution by heating for $10 \mathrm{~min}$ at $80^{\circ} \mathrm{C}$ in $60 \mu \mathrm{L} 1 \times \mathrm{SDS}$ sample buffer (named Elution). For the investigation of the solubility of GST-MBP fusion proteins, cell debris from clarifying the lysate (named Pellet) were resuspended in $60 \mu \mathrm{L} 1 \times$ SDS sample buffer. $15 \mu \mathrm{L}$ of the unbound proteins in the lysate (named Flowthrough) were mixed with $5 \mu \mathrm{L} 4 \times$ SDS sample buffer. All samples were analyzed on a $15 \%$ SDS gel (Ch. 3.2.2.3).

\subsubsection{Small Scale Histone H3 Expression And Purification}

Overnight cultures of E. coli BL21 containing the appropriate plasmid combinations were used to inoculate $500 \mathrm{~mL}$ main cultures to $\mathrm{OD}_{600}$ of 0.1 . The UAAs BocK, BCNK and Nork were supplemented to the main cultures at $\mathrm{OD}_{600}$ of 0.6 to 0.8 to a final concentration of $1 \mathrm{mM}$ and histone $\mathrm{H} 3$ expression was induced by a final concentration of $1 \mathrm{mM}$ IPTG $30 \mathrm{~min}$ after UAA-addition. Cells were pelleted $\left(4^{\circ} \mathrm{C}, 30 \mathrm{~min}, 4,000 \times \mathrm{g}\right) 4$ to $4: 30 \mathrm{~h}$ after induction and pellets were washed with $1 \times$ PBS prior to storage at $-20^{\circ} \mathrm{C}$. Frozen pellets were resuspended in $30 \mathrm{~mL}$ lysis buffer (1× PBS supplemented with 1× PIC, $1 \mathrm{mM}$ PMSF, $1 \mathrm{mM}$ DTT, lysozyme) and incubated at $37{ }^{\circ} \mathrm{C}$ for $30 \mathrm{~min}$ with shaking. DNA was sheared by ultrasonic sound using a sonifier ( $2 \mathrm{~min}$, output: 6 , cycle constant, $4{ }^{\circ} \mathrm{C}$ ) and the lysate was subsequently centrifuged $\left(4^{\circ} \mathrm{C}, 30 \mathrm{~min}, 18,000 \times \mathrm{g}\right)$. The supernatant was discarded and the inclusion bodies containing pellet was resuspended in $1 \times$ PBS supplemented with $1 \%$ Triton X-100 and $1 \mathrm{mM} \mathrm{DTT}$. After centrifugation $\left({ }^{\circ} \mathrm{C}, 30 \mathrm{~min}, 18,000 \times \mathrm{g}\right)$, the pellet was washed ( $1 \times$ PBS with $1 \mathrm{mM} \mathrm{DTT}$ ) and stored at $-20^{\circ} \mathrm{C}$ after centrifugation as before. Frozen pellets were resuspended and macerated in $1 \mathrm{~mL}$ DMSO for $30 \mathrm{~min}$ at $37^{\circ} \mathrm{C}$ with shaking. Afterwards, proteins were extracted from inclusion bodies with the aid of $25 \mathrm{~mL}$ guanidinium solution (6 M guanidinium chloride, $20 \mathrm{mM}$ TRIS ( $\mathrm{pH} 8.0$ ), $2 \mathrm{mM} \mathrm{DTT)}$ for $1 \mathrm{~h}$ at $37^{\circ} \mathrm{C}$ with shaking. After centrifugation as above, the supernatant was transferred into a fresh Falcon tube and $\mathrm{His}_{6}$-tagged $\mathrm{H} 3$ histones were bound in batch to $500 \mu \mathrm{L} \mathrm{of} \mathrm{Ni}^{2+}$-beads with shaking $\left(1 \mathrm{~h}, 4^{\circ} \mathrm{C}\right)$. Beads were washed with $100 \mathrm{~mL}$ wash-buffer $(8 \mathrm{M}$ urea, $100 \mathrm{mM}$ $\mathrm{NaH}_{2} \mathrm{PO}_{4}, 1 \mathrm{mM}$ DTT, pH 6.2) before proteins were eluted with elution-buffer ( $7 \mathrm{M}$ urea, $20 \mathrm{mM} \mathrm{NaOAc}, 200 \mathrm{mM} \mathrm{NaCl}, 1 \mathrm{mM} \mathrm{DTT}$, pH 4.5). All fractions from elution were analyzed 
on 15\% SDS gels (Ch. 3.2.2.3). Fractions containing the desired histones were pooled and dialyzed overnight against water with $5 \mathrm{mM} \beta$-mercaptoethanol (BME; $2 \mathrm{~L}$ ) followed by two dialyses for 1 to $2 \mathrm{~h}$ each. Dialyzed samples were frozen in liquid nitrogen as $500 \mu \mathrm{L}$ aliquots and stored at $-80^{\circ} \mathrm{C}$.

\subsubsection{Large Scale Nanobody NbSyn2 Expression And Purification}

Overnight cultures of $E$. coli BL21 transformed with either pCLA166 + pCLA177 or pCLA174 + pCLA177 were used to inoculate a $500 \mathrm{~mL}$ main culture to $\mathrm{OD}_{600}$ of 0.1 each. Cultures were incubated at $37^{\circ} \mathrm{C}$ for $3 \mathrm{~h}$ and subsequently supplemented with the UAA NorK ( $2 \mathrm{mM}$ final concentration). Nanobody expression was induced by a final concentration of $1 \mathrm{mM}$ IPTG and temperature was shifted to $28^{\circ} \mathrm{C}$ for overnight expression $(\sim 16 \mathrm{~h})$. Samples for western blot were taken before and after induction, normalized to $\mathrm{OD}_{600}$ of 1.5. Cells were pelleted $\left(4{ }^{\circ} \mathrm{C}, 30 \mathrm{~min}, 4,000 \times \mathrm{g}\right)$ and stored at $-20^{\circ} \mathrm{C}$. The remaining medium of both cultures was pooled and filtered using a vacuum filter system with $0.45 \mu \mathrm{m}$ pore size filter papers. The filtered medium was supplemented with $5 \mathrm{mM}$ imidazole, $1.5 \mathrm{mM}$ PMSF, $1 \times \mathrm{PIC}$ and adjusted to $\mathrm{pH} 7.5 . \mathrm{Ni}^{2+}$-beads $(2 \mathrm{~mL})$ were rinsed with washing buffer $(50 \mathrm{mM}$ $\mathrm{NaH}_{2} \mathrm{PO}_{4}, 300 \mathrm{mM} \mathrm{NaCl}, 5 \mathrm{mM}$ imidazole, $\mathrm{pH}$ 7.5) two times and added to the medium. Binding of $\mathrm{His}_{6}$-tagged nanobodies was allowed to occur overnight at $4{ }^{\circ} \mathrm{C}$ with stirring. Beads were pelleted and two times washed with $50 \mathrm{~mL}$ washing buffer at $4{ }^{\circ} \mathrm{C}$ for $15 \mathrm{~min}$ with shaking. Proteins were eluted six times with $1.5 \mathrm{~mL}$ elution buffer $\left(50 \mathrm{mM} \mathrm{NaH}_{2} \mathrm{PO}_{4}\right.$, $300 \mathrm{mM} \mathrm{NaCl}, 500 \mathrm{mM}$ imidazole, $\mathrm{pH} 7.5$ ) at $4{ }^{\circ} \mathrm{C}$ for $10 \mathrm{~min}$ with shaking. All fractions from elution were analyzed on a $15 \%$ SDS gel (Ch. 3.2.2.3). Fractions containing the desired nanobodies were pooled and concentrated to $500 \mu \mathrm{L}$ using centrifugal filter units.

\subsubsection{Expression And Purification of Isotopically Labeled sfGFP}

An overnight culture of E. coli BL21 transformed with pCLA96 and pCLA105, grown in LB medium, was used to inoculate a main culture of $250 \mathrm{~mL}$ M9 minimal medium supplemented with ${ }^{15} \mathrm{~N}$ labeled ${ }^{15} \mathrm{NH}_{4} \mathrm{Cl}$ to $\mathrm{OD}_{600}$ of 0.2 . Cells were incubated at $37^{\circ} \mathrm{C}$ for $7.5 \mathrm{~h}$ and pelleted $\left(4^{\circ} \mathrm{C}, 30 \mathrm{~min}, 4,000 \times g\right)$ at $\mathrm{OD}_{600}$ of $\sim 1.0$. Samples for western blot were taken from overnight and main culture, normalized to $\mathrm{OD}_{600}$ of 1.5. Pelleted cells were washed with $1 \times$ PBS prior to storage at $-20^{\circ} \mathrm{C}$. Frozen cells were resuspended and lysed in $15 \mathrm{~mL}$ lysis buffer $\left(\mathrm{Ni}^{2+}\right.$-wash buffer $(20 \mathrm{mM}$ TRIS- $\mathrm{HCl}, 250 \mathrm{mM} \mathrm{NaCl}, 10 \mathrm{mM}$ imidazole, 
$\mathrm{pH}$ 8.0) supplemented with $1 \times \mathrm{PIC}, 1 \mathrm{mM}$ PMSF, lysozyme, $0.5 \mathrm{mg} / \mathrm{mL}$ DNasel) for $1 \mathrm{~h}$ on ice. Undigested DNA was sheared by ultrasonic sound using a sonifier and the lysate was subsequently clarified by centrifugation $\left(4^{\circ} \mathrm{C}, 30 \mathrm{~min}, 16,100 \times \mathrm{g}\right)$. $\mathrm{His}_{6}$-tagged proteins from the lysate were bound in batch to $500 \mu \mathrm{L}$ of $\mathrm{Ni}^{2+}$-beads with agitation $\left(1 \mathrm{~h}, 4^{\circ} \mathrm{C}\right)$. Beads were washed with $50 \mathrm{~mL}$ cold $\mathrm{Ni}^{2+}$-wash buffer, before elution with four times $1 \mathrm{~mL}$ $\mathrm{Ni}^{2+}$-elution buffer ( $20 \mathrm{mM}$ TRIS-HCl, $250 \mathrm{mM} \mathrm{NaCl}, 200 \mathrm{mM}$ imidazole, $\mathrm{pH}$ 8.0). All fractions from elution were analyzed on a 15\% SDS gel (Ch. 3.2.2.3). Fractions containing the desired sfGFP were pooled, concentrated to $400 \mu \mathrm{L}$ using centrifugal filter units and simultaneously dialyzed against M9 minimal medium without glucose.

\subsubsection{Protein Expression For In-Cell NMR Measurements}

Overnight cultures of $E$. coli BL21 containing the appropriate plasmid combinations, grown in LB medium, were used to inoculate a main culture of $250 \mathrm{~mL} \mathrm{M9} \mathrm{minimal} \mathrm{medium} \mathrm{with}$ unlabeled $\mathrm{NH}_{4} \mathrm{Cl}$ to $\mathrm{OD}_{600}$ of 0.2 . Cells were incubated at $37^{\circ} \mathrm{C}$ until they reached $\mathrm{OD}_{600}$ of 0.6 to 0.8 , pelleted $(\mathrm{RT}, 20 \mathrm{~min}, 4,000 \times \mathrm{g}$ ) and resuspended in M9 medium supplemented with ${ }^{15} \mathrm{~N}$ labeled ${ }^{15} \mathrm{NH}_{4} \mathrm{Cl}$ and $150 \mu \mathrm{g} / \mathrm{mL}$ spectinomycin. After additional $4 \mathrm{~h}$ of incubation at $37^{\circ} \mathrm{C}$ cells were pelleted again (RT, $20 \mathrm{~min}, 4,000 \times \mathrm{g}$ ) and resuspended in $1 \mathrm{~mL}$ unlabeled M9 medium for NMR analysis (performed by AG Zweckstetter).

\subsubsection{Labeling of Proteins With Fluorophores}

In general, the ratio of protein to dye should be 1:10 (w/w) that means $100 \mu \mathrm{g}$ of protein would be labeled with $10 \mu \mathrm{g}$ dye. All dyes (Table 3.7) were prepared as stock solutions with $10 \mathrm{mg} / \mathrm{mL}$ in DMSO. Here they were used in a ratio of 1:100 $(\mathrm{v} / \mathrm{v})$. Labeling reactions were performed at RT for $1.5-2 \mathrm{~h}$ or at $4{ }^{\circ} \mathrm{C}$ overnight.

For the consecutive double labeling of histone $\mathrm{H} 3,100 \mu \mathrm{L}$ of purified histones with Bock, BCNK or NorK incorporated (Ch. 3.2.2.7) were first labeled with $1 \mu \mathrm{L} \mathrm{Abb.} \mathrm{Star635} \mathrm{at} \mathrm{RT} \mathrm{for}$ $1.5 \mathrm{~h}$. An aliquot of $20 \mu \mathrm{L}$ was kept as "tetrazine only" samples and the rest was dialyzed two times for 30 min against $200 \mathrm{~mL}$ water supplemented with $1 \mathrm{mM}$ TCEP using Slide-ALyzer $^{\circledast}$ Mini Dialysis Units. Additionally, $100 \mu \mathrm{L}$ unlabeled histones were dialyzed for "maleimide only" samples. Next, dialyzed unlabeled and single labeled samples were mixed with $1 \mu \mathrm{L}$ Atto565 at $4{ }^{\circ} \mathrm{C}$ overnight. Unbound dyes were removed from all samples by SDS- 
PAGE and the gel was scanned with a Typhoon imager to detect labeled proteins prior to Coomassie staining.

The maleimide conjugated dye, Atto565, was excited by the green laser $(532 \mathrm{~nm})$ and emitted light passed through a $580 \mathrm{~nm}$ band-pass $30 \mathrm{~nm}$ (580 BP 30) filter. For all tetrazine conjugated fluorophores the red laser $(633 \mathrm{~nm})$ was used in combination with a $670 \mathrm{~nm}$ band-pass $30 \mathrm{~nm}$ (670 BP 30) filter. Sensitivity was set to normal and the voltage for the photomultiplier tube (PMT) had to be adapted for each blot individually. The resolution for the most scans was $200 \mu \mathrm{m}$ per pixel, in some cases it was increased to $100 \mu \mathrm{m}$ per pixel.

\subsubsection{Fluorescence Measurement With A Plate Reader}

A FLUOstar Omega (BMG Labtech) plate reader was used to measure fluorescence from sfGFP in intact cells. Samples from E. coli DH10B containing the appropriate plasmid combinations to express SfGFP, from genes with WT sequence or harboring an amber and/or a frameshift codon, were taken with intent to being normalized to $\mathrm{OD}_{600}$ of 0.5 by pelleting ( $3 \mathrm{~min}, 16,100 \times g$ ) and resuspending in $1 \mathrm{~mL} 1 \times$ PBS. $200 \mu \mathrm{L}$ of this cell suspension was transferred into one well of a 96-well black microplate. $200 \mu \mathrm{L} 1 \times$ PBS was used as a reference. The fluorescent signals from GFP were measured using the self-written program "GFP_ORBS-REPORTER" (Plate mode settings: No. of flashes per well: 10; Scan mode: Orbital averaging; Scan diameter $[\mathrm{mm}]$ : 3; Optic Settings: Excitation $485 \mathrm{~nm}$; Emission: $520 \mathrm{~nm}$; Gain: variable; General settings: Top optic used; Positioning delay [s]: 0.2). Kinetic non-endpoint measurments over time were performed using the program "GFP-NMR" that only differs in the number of measuring points.

\subsubsection{Fluorescence-Activated Cell Sorting}

Fluorescence-activated cell sorting (FACS), established by the workgroup of Herzenberg ${ }^{[129]}$, was done at the MRC Laboratory of Molecular Biology, Cambridge, using the cell sorter iCyt Synergy (Sony Biotechnology). It was used for sorting living E.coli based on the fluorescence intensity of sfGFP within the cells. Therefore, $10 \mathrm{~mL}$ aliquots normalized to $\mathrm{OD}_{600}$ of 1.0 were prepared from $100 \mathrm{~mL}$ overnight cultures of $E$. coli $\mathrm{DH} 10 \mathrm{~B}$ containing the appropriate plasmid combinations to express sfGFP, from genes with WT sequence or harboring an amber and a frameshift codon (see Figure 4.34). Cells were pelleted $\left(4^{\circ} \mathrm{C}\right.$, $15 \mathrm{~min}, 4,147 \times \mathrm{g})$ and three times washed with two-fold filtered (syringe filters $(0.2 \mu \mathrm{m})$ ) 
$1 \times$ PBS. Cell suspensions were diluted to $10^{5}$ cells prior to use in the FACS machine, which was finally calibrated to test 30,000 cells per second. Sorted positive clones were temporarily stored in $200 \mu \mathrm{L} \mathrm{SOC}$ medium. For recovery an additional $1 \mathrm{~mL} \mathrm{SOC}$ medium was added and cells were incubated at $37^{\circ} \mathrm{C}$ for $1 \mathrm{~h}$. Recovered cells were transferred into $500 \mathrm{~mL}$ LB medium containing the appropriate antibiotics, in order to prepare glycerol stocks $(500 \mu \mathrm{L}$ cell culture with $500 \mu \mathrm{L}$ glycerol (50\%)) and to isolate DNA (Ch. 3.2.3.1).

\subsubsection{Nucleic Acid Biochemical Methods}

\subsubsection{Preparation of Plasmid DNA}

Plasmid DNA was isolated and purified with the help of kit systems (Ch. 3.1.11) according to manufacturer's manual. In general, bacteria were transformed with the desired plasmid (Ch. 3.2.1.3) and used to inoculate an overnight culture $\left(5 \mathrm{~mL} \mathrm{LB}, 37^{\circ} \mathrm{C}\right)$ containing a selective antibiotic. Up to $4 \mathrm{~mL}$ of this culture were harvested by centrifugation $(16,100 \times g$, $\mathrm{RT}, 3 \mathrm{~min}$ ) and disrupted by alkaline lysis using the kit buffers. The purification of the DNA was performed over silica columns.

\subsubsection{Restriction Enzyme Digestion}

Digestion of DNA was performed by restriction enzymes (Table 3.4) following manufacturer's protocols. These enzymes are endonucleases which operate by recognizing a specific sequence of nucleotides, varying between four and eight base pairs in length, and often palindromic, followed by producing double strand breaks in the $\mathrm{DNA}^{[130]}$. Thereby, some enzymes create overhanging ends (sticky) and some create ends without overhangs (blunt). Categorized into four groups (Typel-IV) based on different traits they differ amongst others in the position of the cleavage site relative to the recognition sequence.

Typell endonucleases, which were used in this thesis, cut their substrate near or inside of their recognition sequence. This makes them easier to handle, compared to randomly cutting enzymes, and thereby the most commonly available and used restriction enzymes. Due to the discovery of new enzymes that belonged to this class, but did not fulfill all classical criteria, a number of subfamilies were founded. One of these enzymes, Dpnl, is able to recognize and cleave methylated and hemimethylated $\mathrm{DNA}^{[131]}$. Therefore, it was used to remove parental template DNA from PCR reactions (Ch. 3.2.3.8, 3.2.3.10 \& 
3.2.3.11). In most cases Dpnl was supplemented as a fortieth of the PCR volume. If the PCR was purified before (Ch. 3.2.3.9) 10x reaction buffer was added (as a tenth of the volume) as well, otherwise the buffer of the PCR was also used for the digest that usually was performed for $1.5 \mathrm{~h}$ at $37^{\circ} \mathrm{C}$.

The majority of the other endonucleases were either used to clone specific DNA fragments into a certain vector backbone (preparative digest) or to conduct test digests (Table 3.10) of prepared plasmid DNA (Ch. 3.2.3.1) from re-transformations or cloning procedures. Digests, especially double digests, were carried out in buffers and relation of enzymes recommended by the manufacturer.

Table 3.10: Pipetting scheme for standard test and preparative digests

\begin{tabular}{llrl}
\multicolumn{2}{c}{ Test digest } & \multicolumn{2}{c}{ Preparative digest } \\
\hline $1 \mu \mathrm{L}$ & buffer, 10x & $2.5 \mu \mathrm{L}$ & buffer, 10x \\
$2 \mu \mathrm{L}$ & DNA & $8 \mu \mathrm{L}$ & DNA ( 1 $\mu \mathrm{g})$ \\
$1 \mu \mathrm{L}$ & enzyme(s) & $2.5 \mu \mathrm{L}$ & enzyme(s) \\
$6 \mu \mathrm{L}$ & $\mathrm{H}_{2} \mathrm{O}$ & $12 \mu \mathrm{L}$ & $\mathrm{H}_{2} \mathrm{O}$ \\
\hline \hline
\end{tabular}

$2 \mathrm{~h}, 37^{\circ} \mathrm{C}$

$3 \mathrm{~h}, 37^{\circ} \mathrm{C}$

Digests for cloning (preparative ones) were treated with alkaline phosphatases (0.5-1 $\mu \mathrm{L}$, $37^{\circ} \mathrm{C}, 0.5-1 \mathrm{~h}$, Table 3.4) afterwards, to remove 5'- (and 3'- for SAP) phosphate groups from digested vectors to prevent self-ligation.

Phosphatases and restriction enzymes were inactivated by heating $\left(65-80{ }^{\circ} \mathrm{C}, 15 \mathrm{~min}\right)$ and/or separation on an agarose gel (Ch. 3.2.3.3), which was also used to separate DNA fragments from digest itself.

\subsubsection{Agarose Gel Electrophoresis}

Agarose gel electrophoresis was performed to analyze size and abundance of DNA (fragments) from restriction digests (Ch. 3.2.3.2) and PCRs (Ch. 3.2.3.8, 3.2.3.10 \& 3.2.3.11) as well as to purify DNA as necessary.

Depending on the expected size of the DNA, different percentages of agarose gels were prepared (Table 3.11). The appropriate amount of agarose was melted and completely dissolved in $0.5 \times$ TBE buffer (Table 3.6) in a microwave, casted into an electrophoresis 
chamber and supplemented with "gel red" in a concentration of 1:50,000 (v/v) after cooling to $50-60{ }^{\circ} \mathrm{C}$.

DNA samples were mixed with 10x DNA loading buffer (Table 3.6) and loaded onto the gel together with a DNA ladder (Ch. 3.1.4) to estimate the size of the DNA. Electrophoresis was performed at $200 \mathrm{~V}, 80-100 \mathrm{~mA}$ at RT with $0.5 \times$ TBE running buffer until the bromphenol blue dye migrated the length of the gel (1-2 h).

Separated DNA bands were visualized by UV light due to the intercalated "gel red" using a gel documentation machine or a UV table. If DNA was needed for further steps, like cloning (Ch. 3.2.3.4), wavelength of the UV light was set to $365 \mathrm{~nm}$ instead of $254 \mathrm{~nm}$.

Table 3.11: Percentages of agarose gels depending on the size of the DNA (fragments)

\begin{tabular}{rc} 
DNA (fragment) size [bp] & [\%] agarose in gel $(w / v)$ \\
\hline$<500$ & 1.5 \\
$200-3,500$ & 1.0 \\
$700-\geq 10,000$ & 0.7
\end{tabular}

\subsubsection{Extraction of DNA From Agarose Gels}

DNA required for cloning was preparatively digested (Ch. 3.2.3.2) and subsequently separated via agarose gel electrophoresis (Ch. 3.2.3.3). Visualized on a UV table ( $365 \mathrm{~nm}$ ), those bands of correct size were sliced out of the gel with a scalpel. Afterwards, the DNA was extracted using a gel extraction kit (Ch. 3.1.11) according to manufacturer's manual. The purified DNA was eluted from the silica columns with max. $30 \mu \mathrm{L}$ of elution buffer and stored at $-20^{\circ} \mathrm{C}$ or directly used for ligations (Ch. 3.2.3.6)

\subsubsection{Separation of Multiple Plasmids}

Two methods were used for the separation of multiple plasmids isolated from cells (Ch. 3.2.3.1) transformed with more than one plasmid. First, plasmids were separated on agarose gels (Ch. 3.2.3.3) and DNA/plasmid of expected size extracted from gel (Ch. 3.2.3.4), subsequently. Second, unwanted plasmids were destroyed by restriction enzyme digestion (Ch. 3.2.3.2) prior to transformation of E. coli cells. Plasmids of interest were isolated from transformed cells, afterwards. 


\subsubsection{Ligation of Two Double-Stranded DNA Fragments}

A double-stranded DNA fragment was typically digested with two different restriction enzymes (Ch. 3.2.3.2) and was ligated with another double-stranded DNA fragment, digested with the same enzymes, using the T4 DNA ligase. Therefore, a 3-5 fold molar excess of the smaller fragment, e.g., a gene for a protein, called the insert is mixed with the bigger fragment, commonly a vector backbone (Table 3.12). For a negative control water replaced the insert DNA. The ligation was carried out for $2 \mathrm{~h}$, at RT, or more frequently at $16^{\circ} \mathrm{C}$ overnight. The product of the ligation reaction $(5-10 \mu \mathrm{L})$ was directly used for transformation (Ch. 3.2.1.3) and the success of the ligation was verified by test digests (Ch. 3.2.3.2) and/or sequencing (Ch. 3.2.3.13).

Table 3.12: Pipetting scheme for a standard ligation reaction mix

\begin{tabular}{rc} 
& Volume $[\mu \mathrm{L}]$ \\
\hline T4 Ligase buffer, 10x & 1 \\
Vector DNA (big fragment) & 2 \\
Insert DNA (small fragment)/ $\mathrm{H}_{2} \mathrm{O}$ & 6.5 \\
T4 DNA ligase & 0.5 \\
\hline
\end{tabular}

\subsubsection{Ethanol Precipitation}

Ethanol precipitations were performed to remove salts prior to transformation, if cells needed to be transformed with ligation reactions via electroporation due to required higher efficiencies, and/or to reduce the volume of large scale ligations. For this purpose, the ligation product was mixed in a ratio of $10: 1(\mathrm{v} / \mathrm{v})$ with $3 \mathrm{M}$ sodium acetate solution $(\mathrm{pH}$ 5.2) and subsequently supplemented with absolute $\mathrm{EtOH}$ equal to double the volume of the whole reaction mixture. After an incubation period of $30 \mathrm{~min}$ on ice the DNA was pelleted by centrifugation $\left(16,100 \times \mathrm{g}, 4^{\circ} \mathrm{C}, 15 \mathrm{~min}\right)$. The pellet was washed two times with $70 \% \mathrm{EtOH}$ equal to twice the starting volume of the ligation product and centrifuged each time as before. The supernatant was discarded and the DNA pellet was air dried for $5 \mathrm{~min}$ at RT. After resuspending the DNA in a small amount of $d_{d H_{2}} \mathrm{O}$ or TE buffer (Table 3.6) an incubation for $10 \mathrm{~min}$ at $60^{\circ} \mathrm{C}$ followed to dissolve the pellet completely. DNA could then be stored at $-20^{\circ} \mathrm{C}$ or used for electroporation (Ch. 3.2.1.4). 


\subsubsection{Polymerase Chain Reaction}

The polymerase chain reaction (PCR) is a technique used to specifically and exponentially amplify a piece of DNA. Since this method, developed by Kary Mullis ${ }^{[132]}$, relies on thermal cycling with periods of heating and cooling, necessary for DNA melting and enzymatic replication, thermostable enzymes like Taq or Phusion DNA polymerases (Table 3.4) are essential. In general, a PCR consists of 6 different steps in which step two to four are repeated in 20-40 cycles.

1. Initial denaturation: In this step the reaction is heated up to $94-96{ }^{\circ} \mathrm{C}$ to ensure that the template DNA is completely melted which is important for step three, the annealing of the primers. Additionally, it activates DNA polymerases that need heat activation (hot-start PCR) ${ }^{[133]}$.

2. Cyclic denaturation: The purpose of this step is the same as for the first one, melting the DNA (template and new synthesized strands) by heating to $94-96^{\circ} \mathrm{C}$ to obtain single-stranded DNA but in a cyclic manner.

3. Cyclic annealing: The temperature of the reaction is lowered to $50-60^{\circ} \mathrm{C}$, usually $5^{\circ} \mathrm{C}$ lower than the melting temperature of the primers, singlestranded oligonucleotides that serve as a starting point for the polymerase. The low temperature allows the primers, which are complementary to the template DNA, flanking the sequence that should be amplified, to hybridize.

4. Cyclic elongation: In this step the polymerase synthesizes a new complementary DNA strand starting at a primer by adding dNTPs. Therefore, the temperature is changed to the appropriate optimum for the used polymerase $\left(68-72^{\circ} \mathrm{C}\right)$. The elongation time depends on the length of DNA to be amplified and on the speed of the particular polymerase.

5. Final elongation: All remaining single-stranded DNA fragments are fully extended during this step at the same temperature used in step 4.

6. Stop/cooling: The reaction mix is cooled down to "stop" enzymatic reactions and for short-term storage.

The standard course for a PCR is shown in Table 3.13 and the composition for one PCR reaction mix is shown in Table 3.14. 
Table 3.13: Standard course of a PCR

\begin{tabular}{lcc} 
Step & Temperature $\left[{ }^{\circ} \mathrm{C}\right]$ & Duration $[\mathrm{s}]$ \\
\hline 1. Initial denaturation & 95 & 90 \\
2. Cyclic denaturation & 95 & 30 \\
3. Cyclic annealing & 56 & 30 \\
4. Cyclic elongation & 72 & $60 / 1 \mathrm{kbp}$ \\
5. Final elongation & 72 & Step $4+60$ \\
6. Stop/cooling & 4 & $\infty$ \\
\hline
\end{tabular}

Table 3.14: Pipetting scheme for the composition of one PCR reaction mix

\begin{tabular}{rc} 
& Volume $[\mu \mathrm{L}]$ \\
\hline Taq buffer with $\mathrm{KCl}, 10 \times$ & 5 \\
dNTPs (10 mM each), 50x & 1 \\
Primer mix (for + rev), $10 \mu \mathrm{M}$ & 4 \\
$\mathrm{MgCl}_{2}, 25 \mathrm{mM}$ & 4 \\
Template DNA & 1 \\
Taq DNA polymerase & 1 \\
$\mathrm{H}_{2} \mathrm{O}$ & 34 \\
\hline
\end{tabular}

Before further usage, PCR products were purified using EtOH precipitation (Ch. 3.2.3.7) or kit systems (Ch. 3.2.3.9).

\subsubsection{Purification of PCR Products}

For the removal of enzymes, unused primers, dNTPs and salts, PCR reactions were purified using EtOH precipitation (Ch. 3.2.3.7) or kit systems (Ch. 3.1.11) according to manufacturer's manual. The cleaned up DNA was eluted from the silica columns with max. $30 \mu \mathrm{L}$ of elution buffer and stored at $-20^{\circ} \mathrm{C}$ or directly used, e.g., restriction digests (Ch. 3.2.3.2). 


\subsubsection{QuikChange PCR}

A QuikChange ( $Q C$ ) is a site-directed mutagenesis (developed by Agilent, Böblingen former Stratagene) that allows to easily carry out vector modifications (Figure 3.1).

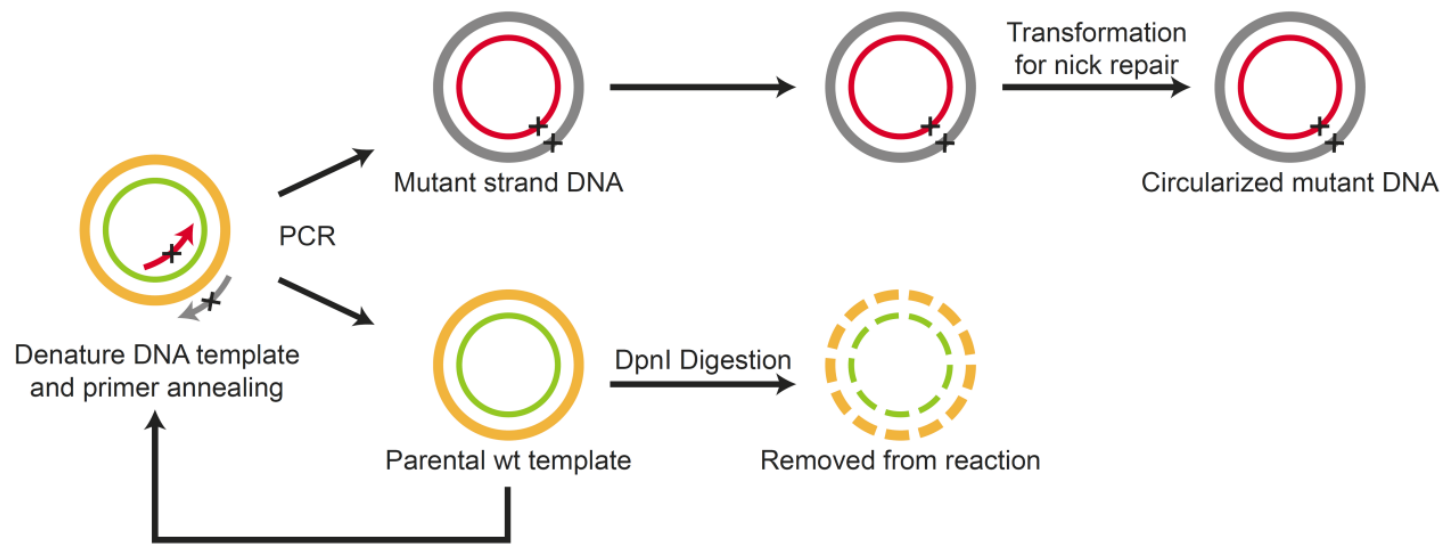

Figure 3.1: Schematic view of QuikChange cycles.

Figure is adapted from QuikChange II XL site-directed mutagenesis protocol (Agilent). Mutant strand DNA (grey and red rings) is synthesized by performing thermal cycling (PCR) to denature the DNA template (orange and green rings) and to anneal mutagenic primers (grey and red arrows) containing the desired mutations (black crosses). Primers are extended with Pfu Turbo DNA polymerase. Parental methylated and hemimethylated template DNA is digested by Dpnl. Finally, competent cells are transformed with the mutated molecule for nick repair.

Since these PCR reactions amplify an entire vector a high fidelity proofreading polymerase, $P f u$ Turbo, was required. Primers were designed so that forward and reverse primers both included the desired mutations in a complementary sequence of at least $25 \mathrm{bp}$. The $3^{\prime}$-ends of each primer were complementary to a minimum of 10-15 bp of the vector backbone to allow annealing to the correct positions. The composition for one QC-PCR reaction mix is shown in Table 3.15 .

Table 3.15: Pipetting scheme for the composition of one QuikChange PCR reaction mix

Volume $[\mu \mathrm{L}]$

\begin{tabular}{rc}
\hline$f u$ buffer, 10x & 2 \\
dNTPs (10 mM each), 50x & 0.4 \\
Pfu Turbo DNA polymerase & 0.4 \\
Template DNA & 0.4 \\
Primer mix (for + rev), $10 \mu \mathrm{M}$ & 0.8 \\
$\mathrm{H}_{2} \mathrm{O}$ & 16 \\
\hline
\end{tabular}


The standard course for a $Q C$ is shown in Table 3.16. Compared to a common PCR (Ch. 3.2.3.8) fewer cycles were used to avoid the amplification of random mutations. The DNA template was digested with $\mathrm{Dpnl}(0.5 \mu \mathrm{L})$ subsequent to PCR $\left(37^{\circ} \mathrm{C}, 1-1.5 \mathrm{~h}\right)$, as depicted in Figure 3.1 , followed by transformation of $E$. coli cells with $5-10 \mu l$ of the mixture (Ch. 3.2.1.3). The modified vectors were isolated from those cells (Ch. 3.2.3.1) and the success of the modification determined by sequencing (Ch. 3.2.3.13).

Table 3.16: Standard course of a QC-PCR

\section{Step}

1. Initial denaturation

2. Cyclic denaturation

3. Cyclic annealing

4. Cyclic elongation

5. Final elongation

6. Stop/cooling

\section{Temperature $\left[{ }^{\circ} \mathrm{C}\right]$}

96

95

56

72

72

4

\section{Duration [s]}

60

20

30

90/1kbp

Step $4+60$

$\infty$

\subsubsection{Inverse PCR}

Inverse PCR is a variation of the conventional PCR (Ch. 3.2.3.8) and was originally used for the rapid in vitro amplification of unknown DNA sequences that flank a region of known sequence ${ }^{[134]}$. The restriction enzyme-mediated inverse PCR incorporates unique restriction enzyme sites at the $5^{\prime}$-ends of inverse tail-to-tail primers ${ }^{[135]}$. Similar to the $\mathrm{QC}$ (Ch. 3.2.3.10) this technique can be used for site-directed mutagenesis but with a higher efficiency. The increase in efficiency is due to the primer design, since only one primer needs to hold the desired mutations, and the generation of sticky-ends by unique restriction enzyme digests which improve ligation efficiency of linear mutant DNA strands. This method amplifies an entire vector like the $Q C$ and requires also a high fidelity proofreading polymerase. The Expand High Fidelity Polymerase Mix (Table 3.4) was used for this purpose. The general procedure of the restriction enzyme-mediated inverse PCR is shown in Figure 3.2. 


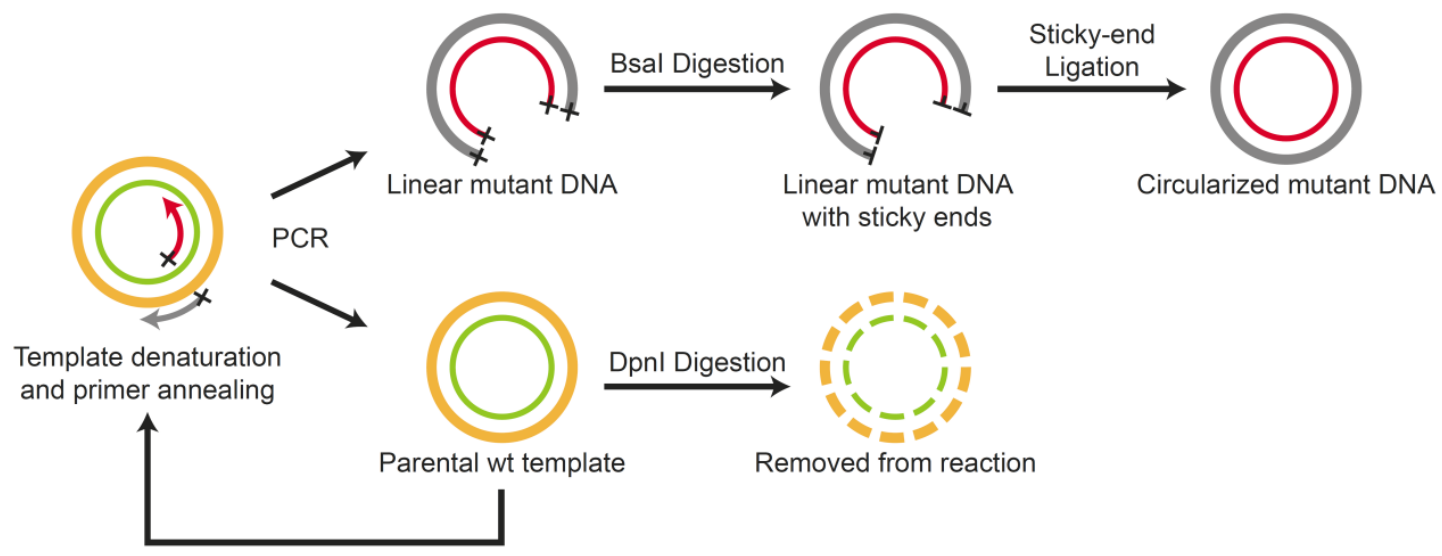

Figure 3.2: Schematic representation of the restriction enzyme-mediated inverse $\mathbf{P C R}^{[135]}$.

Sense and antisense primers (grey and red arrows) are designed in such a way that they contain a unique restriction site, Bsal (black crosses), and anneal to the opposite ends instead of facing each other as usual. Desired mutations were mostly incorporated into the reverse primer, only. The template DNA (orange and green rings) is amplified via PCR, resulting in linear mutated DNA (grey and red rings), and subsequently eliminated by Dpnl digestion. Blunt ends of mutant DNA are made sticky upon digest with Bsal, which improves the ligation efficiency and subsequent circularization of the PCR product.

The composition of one inverse PCR reaction is shown in Table 3.17 and the course in Table 3.18. For the setup of the reaction mix two mixtures were prepared as recommended by Roche's protocol, one with buffer, enzyme and half of the water, the other with the rest. Both mixtures were combined directly before PCR.

Table 3.17: Pipetting scheme for the composition of one inverse PCR reaction mix

\begin{tabular}{rc} 
& Volume $[\mu \mathrm{L}]$ \\
\hline Expand HF Buffer (15 mM MgCl 2 ), 10x & 5 \\
dNTPs (10 mM each), 50x & 1 \\
Template DNA & 1 \\
Primer mix (for + rev), 10 $\mu \mathrm{M}$ & 4 \\
Expand High Fidelity Polymerase Mix & 1 \\
$\mathrm{H}_{2} \mathrm{O}$ & 38 \\
\hline
\end{tabular}

PCR was followed by purification using kit systems (Ch. 3.2.3.9) and digest of parental template with Dpnl from NEB as described in Ch. 3.2.3.2. Afterwards, mutant DNA was digested with Bsal $\left(3 \mathrm{~h}, 50^{\circ} \mathrm{C}\right)$ to achieve sticky-ends. Therefore, DNA concentration was measured (Ch. 3.2.3.12) and $1 \mu \mathrm{L}$ of Bsal per $10 \mu \mathrm{g}$ DNA added to the mixture. Recommended buffer was already supplemented for the Dpnl digest. After cutting the recognition and cutting sites for Bsal (5'-GGTCTC-3'), incorporated at the $5^{\prime}$-ends of the primers, are automatically eliminated and not maintained in the final product. 
Table 3.18: Standard course of a restriction enzyme-mediated inverse PCR.

A touchdown PCR is performed to avoid nonspecific binding of primers and to increase yields significantly ${ }^{[136,137]}$. During the first 10 cycles annealing temperature is decreased by $1{ }^{\circ} \mathrm{C}$ causing the primers to anneal at highest temperature which is least-permissive for nonspecific binding. Therefore, amplification of nonspecific sequences is outcompeted.

Step

1. Initial denaturation

2. Cyclic denaturation

3. Cyclic annealing

4. Cyclic elongation

5. Cyclic denaturation

6. Cyclic annealing

7. Cyclic elongation

8. Final elongation

9. Stop/cooling
Temperature [ $\left.{ }^{\circ} \mathrm{C}\right]$

95

95

65

68

95

55

68

68

4
Duration [s]

60

20
30

$60 / 1,5 \mathrm{kbp}$

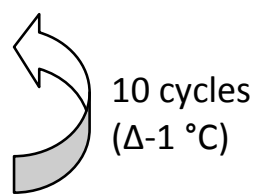

20

30

$60 / 1,5 \mathrm{kbp}$

Step $7+60$

$\infty$

Prior to ligation of the sticky-ends using T4 DNA ligase at $16^{\circ} \mathrm{C}$ overnight $(50 \mu \mathrm{L}$ digested PCR, $35 \mu \mathrm{L} \mathrm{H2O}, 10 \mu \mathrm{L}$ T4 Ligase buffer (10x), $5 \mu \mathrm{L}$ T4 DNA ligase) the digested PCR was purified again with PCR purification kits (Ch. 3.2.3.9).

Ligations were usually precipitated with EtOH (Ch. 3.2.3.7) and dissolved DNA used for transformation via electroporation (Ch. 3.2.1.4). The inoculation of big cultures (about $500 \mathrm{~mL}$ ) followed by the isolation of the modified plasmids allowed the amplification of the DNA for further experiments. A dilution series of these cultures were used to determine the diversity of the PCR. Therefore, $50 \mu \mathrm{L}$ of the inoculated medium was plated directly on agar plates containing the appropriate antibiotic, representing a $10^{4}$ dilution. Further dilutions up to $10^{8}$ were made by diluting previous ones tenfold with medium and also plated on agar plates.

\subsubsection{Measuring Nucleic Acid Concentration}

DNA and RNA concentrations of aqueous solutions were measured by absorption in UV cuvettes using a photometer. $3 \mu \mathrm{L}$ of the nucleic acid containing solution was diluted in $100 \mu \mathrm{L}$ water. Detection occurred at $260 \mathrm{~nm}\left(\mathrm{~A}_{260}\right)$ with pure water $(100 \mu \mathrm{L})$ as a reference. The purity of the nucleic acid was also given by the photometer, calculated by the quotient of $A_{260}$ to $A_{280}$. 


\subsubsection{Sequencing of Plasmids}

DNA sequencing was performed using chain-terminating inhibitors, dideoxynucleotide triphosphates (ddNTPs), developed by Sanger and colleagues ${ }^{[138,139]}$.

This method works similar to a normal PCR (Ch. 3.2.3.8) and needs the same components, i.e., a (single-stranded) DNA template, a primer, a DNA polymerase and normal dNTPs, except the ddNTPs that randomly terminate the extension of the DNA strand due to the lack of the 3'-OH group. The use of ddNTPs each labeled with a different fluorescent dye emitting light of a unique wavelength allows sequencing in a single reaction. At the end of the PCR fragments of all possible lengths are statistically contained in the mixture which can be separated and analyzed by a sequencing machine subsequently.

The sequencing mix BigDye (BigDye ${ }^{\circledast}$ Terminator v1.1 Cycle Sequencing Kit, Applied Biosystems, USA) used for sequencing included the DNA polymerase, normal dNTPs and fluorescent labeled ddNTPs as well. The complete composition for a sequencing PCR is shown in Table 3.19 and the course in Table 3.20.

Table 3.19: Pipetting scheme for the composition of one sequencing PCR reaction mix

\begin{tabular}{rc} 
& Volume $[\mu \mathrm{L}]$ \\
\hline Seq buffer & 1 \\
Seq mix. BigDye & 1 \\
Primer, $10 \mu \mathrm{M}$ & 0.8 \\
Template DNA, 200-400 ng & $1-7.2$ \\
$\mathrm{H}_{2} \mathrm{O}$ & Adjust to $10 \mu \mathrm{L}$
\end{tabular}

Table 3.20: Standard course of a sequencing PCR

\section{Step}

1. Initial denaturation

2. Cyclic denaturation

3. Cyclic annealing

4. Cyclic elongation

5. Final elongation

6. Stop/cooling
Temperature $\left[{ }^{\circ} \mathrm{C}\right] \quad$ Duration $[\mathbf{s}]$

96 96 55

60

60

240

4

240
40

240

60

10

15

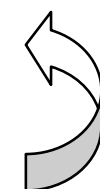

25 cycles 
The DNA products were purified subsequently to the PCR. Therefore, the mixture was transferred to a $1.5 \mathrm{~mL}$ Eppendorf tube, supplemented with $2 \mathrm{mM}$ EDTA- $\mathrm{Na}_{2}, 50 \mathrm{mM}$ sodium acetate and $80 \% \mathrm{EtOH}$ (final concentrations), gently mixed and incubated for $5 \mathrm{~min}$ at RT. After a centrifugation step $(16,100 \times g, R T, 15 \mathrm{~min})$ the supernatant was discarded and the pellet washed with $70 \mu \mathrm{L}$ of $70 \% \mathrm{EtOH}$, followed by a second centrifugation for $5 \mathrm{~min}$. The supernatant was discarded again and the pellet was dried in a speedvac/concentrator for $2 \mathrm{~min}$. The DNA pellet was dissolved in $15 \mu \mathrm{L}$ formamide (99.5\%) and given to AG Pieler, Göttingen for sequencing in a capillary sequencing machine.

\subsubsection{Extraction/Isolation of RNA}

Total RNA was isolated from bacterial cells by guanidine thiocyanate-phenol-chloroform extraction using TRIzol Reagent ${ }^{\circledR}$ (Invitrogen, Darmstadt; registered trademark of Molecular Research Center, Inc.).

TRI Reagent is the improved version of the single-step method developed by Chomczynski and Sacchi ${ }^{[140,141]}$. The guanidine thiocyanate lyses cells and inhibits RNases at the same time. The addition of chloroform (or bromochloropropane) followed by centrifugation splits the mixture into three phases, i.e., an upper aqueous phase containing the RNA, an interphase harboring the DNA and a lower organic phase including the proteins. The RNA is obtained by supplementing isopropanol to the aqueous phase, washed with $\mathrm{EtOH}$ and dissolved subsequently.

The isolation was performed according to manufacturer's manual except for the first step. The lysis of a recommended maximum of $10^{7}$ bacterial cells per $1 \mathrm{~mL}$ of TRI Reagent did not result in a satisfying yield of RNA. Hence, the pellet of $1 \mathrm{~mL}$ bacterial culture with an average $\mathrm{OD}_{600}$ of 3 was lysed with $1 \mathrm{~mL}$ of TRI Reagent. The washed RNA was dissolved in $30 \mu \mathrm{L} \mathrm{H} \mathrm{H}_{2} \mathrm{O}$, finally. 


\subsubsection{Northern Blot}

For the identification of specific tRNAs via northern blot total RNA extracts (Ch. 3.2.3.14) were separated by size using acid urea PAGE conforming to the protocol of Köhrer et al. ${ }^{[142]}$

Table 3.21: Composition of an acid urea polyacrylamide gel for acid urea PAGE

\begin{tabular}{rcc} 
Ingredients & Volume $[\mathrm{mL}]$ & Final Concentration \\
\hline $\mathrm{H}_{2} \mathrm{O}$ & 10 & - \\
$30 \%$ polyacrylamide & 7.2 & $6.5 \%$ \\
$(19: 1$ acrylamid:bisacrylamid) & & \\
$1 \mathrm{M}$ sodium acetate, pH 5.0 & 3.3 & $0.1 \mathrm{M}$ \\
Urea & $16 \mathrm{~g}=11.5 \mathrm{~mL}$ & $8 \mathrm{M}$ \\
$10 \%$ APS (w/v) & 1.2 & $0.36 \%(\mathrm{w} / \mathrm{v})$ \\
$99.9 \%$ TEMED & 0.025 & $0,075 \%(\mathrm{v} / \mathrm{v})$
\end{tabular}

Isolated RNA was mixed 1:1 (v/v) with acid urea sample buffer (Table 3.6) following extraction. Approximately $90 \mathrm{ng}$ of RNA were loaded onto the gel that was subjected to a short pre-electrophoresis for $30 \mathrm{~min}$ in the cold room. Electrophoresis was performed at $168 \mathrm{~V}$ and $165 \mathrm{~mA}(\sim 12 \mathrm{~V} / \mathrm{cm})$ for $150-210 \mathrm{~min}$ in the cold room with $0.1 \mathrm{M}$ sodium acetate $\mathrm{pH} 5.0$ as running buffer until the bromphenol blue dye reached the end of the gel.

As a size standard, low range RNA and oligonucleotide ladders (Ch. 3.1.4) were also loaded onto the gel. In vitro RNA transcripts of the correspondent tRNA synthesized by T7 RNA polymerase (Ch. 3.2.3.16) served as size standard and positive control for northern blots.

For the actual blot only the portion of the gel between both dyes containing small RNAs like tRNAs was used. RNAs were transferred to a nylon membrane using a semidry blotter in a similar way as described in Ch. 3.2.2.4. The transfer was carried out at $25 \mathrm{~V}$ and $513 \mathrm{~mA}$ for 80 min in the cold room with $1 \times$ NB transfer buffer (Table 3.6). Afterwards, the gel was soaked in a "gel red" bath to verify the success of the transfer using a gel documentation machine and the RNA was immobilized through covalent linkage to the membrane by heat ( $2 \mathrm{~h}, 75^{\circ} \mathrm{C}$ in a hybridization oven) or UV light ( $90 \mathrm{~s}, 254 \mathrm{~nm}$ on a UV table).

For northern hybridization, DNA oligonucleotides were designed (Table 8.2) with a length of 17-23 bp, 5'-labeled with Cy3, Cy5 or DIG and complementary to the anticodon stem and loop domain of the desired tRNA target. 
Prior to hybridization a prehybridization in the appropriate solution ( $30 \mathrm{~mL}$, Table 3.6) was executed for $6-12 \mathrm{~h}$ at $42{ }^{\circ} \mathrm{C}$ in a hybridizer oven, with constant rotation. Thereafter, the temperature was increased to $68^{\circ} \mathrm{C}$ and the solution exchanged with hybridization solution (30 mL, Table 3.6). After incubating the membrane for $12-24 \mathrm{~h}$ several washing steps were performed at RT with $6 \times$ SSC (twice for 10 min; low stringency, Table 3.6), followed by washes with $4 \times$ and $2 \times$ SSC (each for 20 min; medium and high stringency).

Northern blots with Cy3 or Cy5 labeled hybridization probes could now directly be utilized for the detection of tRNAs using a Typhoon imager set to the correct laser and filter settings. Cy3 was excited by the green laser $(532 \mathrm{~nm})$ and emitted light passed through a $580 \mathrm{~nm}$ band-pass $30 \mathrm{~nm}$ (580 BP 30) filter. For Cy5 the red laser (633 nm) was used in combination with a $670 \mathrm{~nm}$ band-pass $30 \mathrm{~nm}$ (670 BP 30) filter. Sensitivity was set to normal and the voltage for the photomultiplier tube (PMT) had to be adapted for each blot individually. The resolution for the most scans was $200 \mu \mathrm{m}$ per pixel, in some cases it was increased to $100 \mu \mathrm{m}$ per pixel.

For the detection of DIG-labeled probes further steps were necessary (all carried out at RT). First, the membrane was equilibrated with $30 \mathrm{~mL}$ buffer 1 (Table 3.6) for 5-10 min, followed by blocking with $20 \mathrm{~mL}$ buffer 2 (Table 3.6) for $30 \mathrm{~min}$ and an incubation with anti-DIG-APantibody in buffer 2 for $30-60$ min (or overnight at $4{ }^{\circ} \mathrm{C}$ ). Unbound antibodies were removed by washing three times for 10 min with $30 \mathrm{~mL}$ buffer 1 . The membrane was then equilibrated with $10 \mathrm{~mL}$ buffer 3 (Table 3.6) for $10 \mathrm{~min}$ and the chemiluminescence reaction started subsequently by adding $1 \mathrm{~mL}$ buffer 3 containing $5 \mu \mathrm{L} C D P$-Star directly onto the membrane. After 5 min of membrane incubation with the substrate, in an X-ray cassette, emitted light was captured on ECL films for several seconds, to minutes, until the desired band intensities were achieved. The films were developed in an automatic X-ray film processor.

\subsubsection{Transcription With T7 RNA Polymerase}

Positive controls for northern blots (Ch. 3.2.3.15) were made via T7 RNA polymerase (T7RP) transcription. First, a PCR (Ch. 3.2.3.8) was performed to build a template that contained the recognition site for the T7 polymerase, 5'-TAATACGACTCACTATA-3', and the gene for the appropriate tRNA. The success of the PCR was verified by electrophoresis on an agarose gel (Ch. 3.2.3.3) and the PCR product was purified (Ch. 3.2.3.9). 
To destroy remaining RNases, the mixture was treated with proteinase $\mathrm{K}(1 \mu \mathrm{L}$ per $50 \mu \mathrm{L}$ $\mathrm{PCR}, 30 \mathrm{~min}, 50-55^{\circ} \mathrm{C}$ ). Inactivation of the proteinase $\mathrm{K}$ was carried out at $95^{\circ} \mathrm{C}$ for $10 \mathrm{~min}$.

The transcription was performed at $37^{\circ} \mathrm{C}$ for $2 \mathrm{~h}$. The composition for one reaction is shown in Table 3.22; components were received from AG Ficner, Göttingen. The mixture was set up at RT due to the spermidine in the buffer which causes negative effects in the cold $^{[143,144]}$.

Table 3.22: Pipetting scheme for the composition of one transcription reaction mix

\begin{tabular}{rc} 
& Volume $[\mu \mathrm{L}]$ \\
\hline $\mathrm{MgCl}_{2}, 100 \mathrm{mM}$ & 4.5 \\
rNTPs (40 mM each) & $20(4 \times 5)$ \\
Transcription buffer, 10× & 5 \\
Template DNA & 18 \\
T7 RNA polymerase (1 mg/mL) & 2.5 \\
\hline
\end{tabular}

Transcription was stopped by the addition of $5 \mu \mathrm{L} 0.5 \mathrm{M}$ EDTA- $\mathrm{Na}_{2}$ and the mixture subsequently centrifuged to remove generated pyrophosphates $(16,100 \times g, \mathrm{RT}, 3 \mathrm{~min})$. The supernatant containing the RNA oligos was purified (Ch. 3.2.3.9), and the elution mixed with acid urea sample buffer (Table 3.6) and boiled at $94{ }^{\circ} \mathrm{C}$ for $5 \mathrm{~min}$ to denature the RNA. Table 3.23 gives an overview of transcripts produced.

Table 3.23: Overview of RNA transcripts made by T7 RNA polymerase.

Transcripts are listed with the primers used for the PCR to produce the T7 polymerase recognition site containing template.

\begin{tabular}{lll} 
Transcript & Primer & Chapter \\
\hline T7 PyIT & C28 and C29 & 4.1 .2 \\
T7 SerT & C30 and C31 & 4.1 .2 \\
T7 TyrT & C42 and C43 & 4.1 .4 \\
\hline
\end{tabular}




\subsubsection{Reporter Assays}

\subsubsection{Chloramphenicol Reporter Assay}

The chloramphenicol $(\mathrm{Cm})$ reporter assay (Figure 3.3$)$ was the standard evaluation procedure to classify the suppression efficiency of a given system. The more resistant the cells were to $\mathrm{Cm}$, the more efficient the suppression worked. A system that had insufficient suppression activity led to cell death. The $\mathrm{Cm}$ assay could be utilized to evolve synthetases for new UAAs, for example, or to investigate optimizations on components of the genetic code expansion machinery.

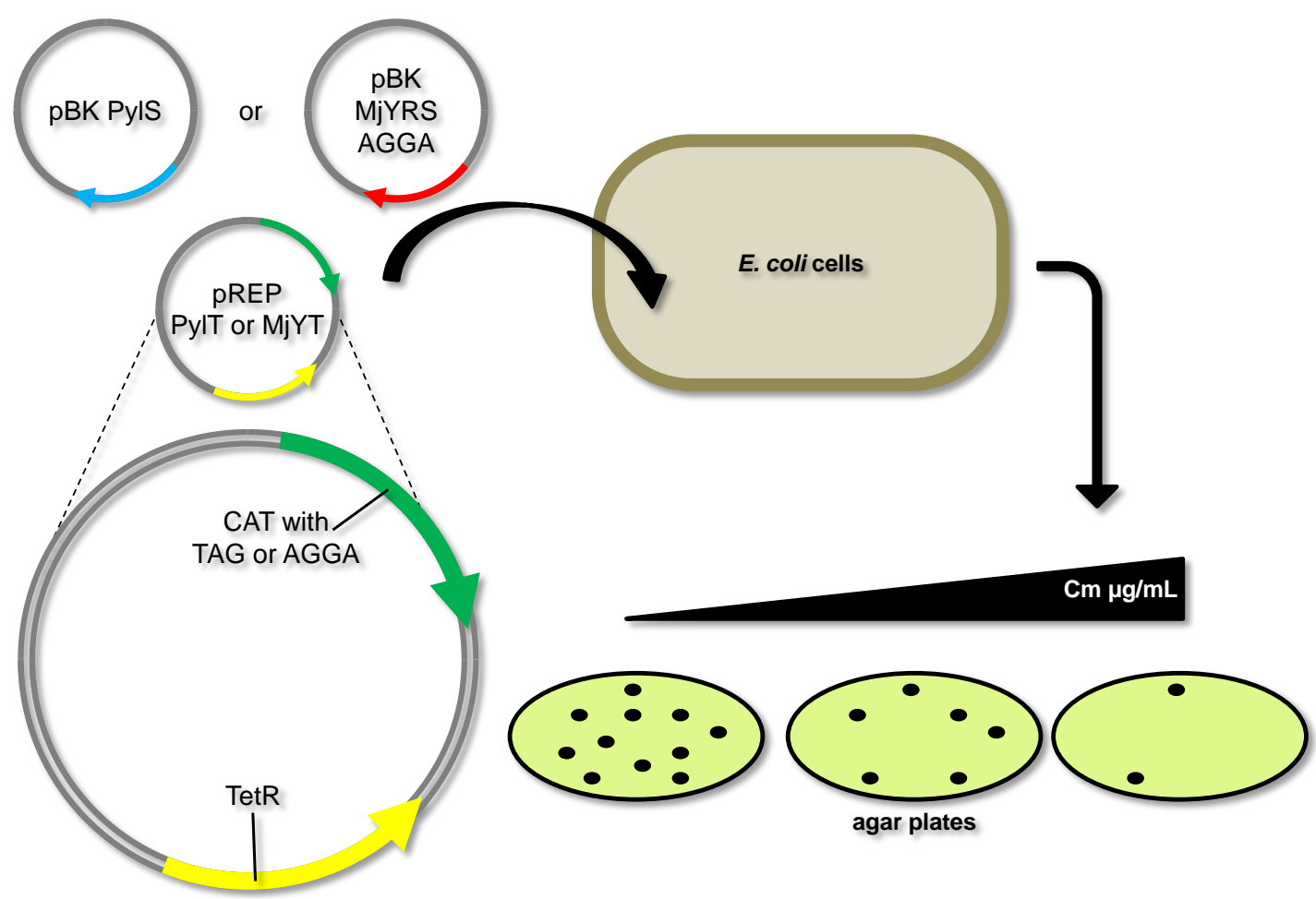

Figure 3.3: Schematic view of the chloramphenicol reporter assay.

A synthetase containing plasmid, derived from pBK PylS ${ }^{[95]}$ (pCLA1) or pBK MjYRS_TAG/AGGA ${ }^{[45,78,83]}$ (pCLA2/pCLA3), was combined with the corresponding pREP ${ }^{[78,95,145]}$ reporter plasmid harboring the gene for PyIT (pCLA4) or MjYT (pCLA5/pCLA6; Table 8.1), respectively. In addition to the gene for the tRNA, the plasmid also comprised a gene for a chloramphenicol acetyltransferase (CAT) with either a TAG or an AGGA codon. E. coli cells, ususally DH10B, were transformed with both plasmids (Ch. 3.2.1.3 and 3.1.9) and plated on agar plates with an increasing concentration of chloramphenicol, appropriate antibiotics for the plasmids and if required relevant UAAs. The better a plasmid combination worked, the higher the concentration of $\mathrm{Cm}$ the cells were able to survive. Resistance genes on plasmids are indicated by the arrows: blue = Kan; red = Amp; green $=\mathrm{Cm}$; yellow $=$ Tet. 


\subsubsection{GFP Reporter Assay}

The GFP reporter assay (Figure 3.4) was another option for quantifying the suppression efficiency of a given system. In contrast to the $\mathrm{Cm}$ reporter (Ch. 3.2.4.1), weak systems did not cause cell death. The advantage of this reporter was due to the fluorescent trait of GFP, which could be easily and directly measured using a plate reader (Ch. 3.2.2.12). Since all GFP reporter plasmids contained a $\mathrm{His}_{6}$-tagged GFP, detection via western blot (Ch. 3.2.2.4) was also possible.

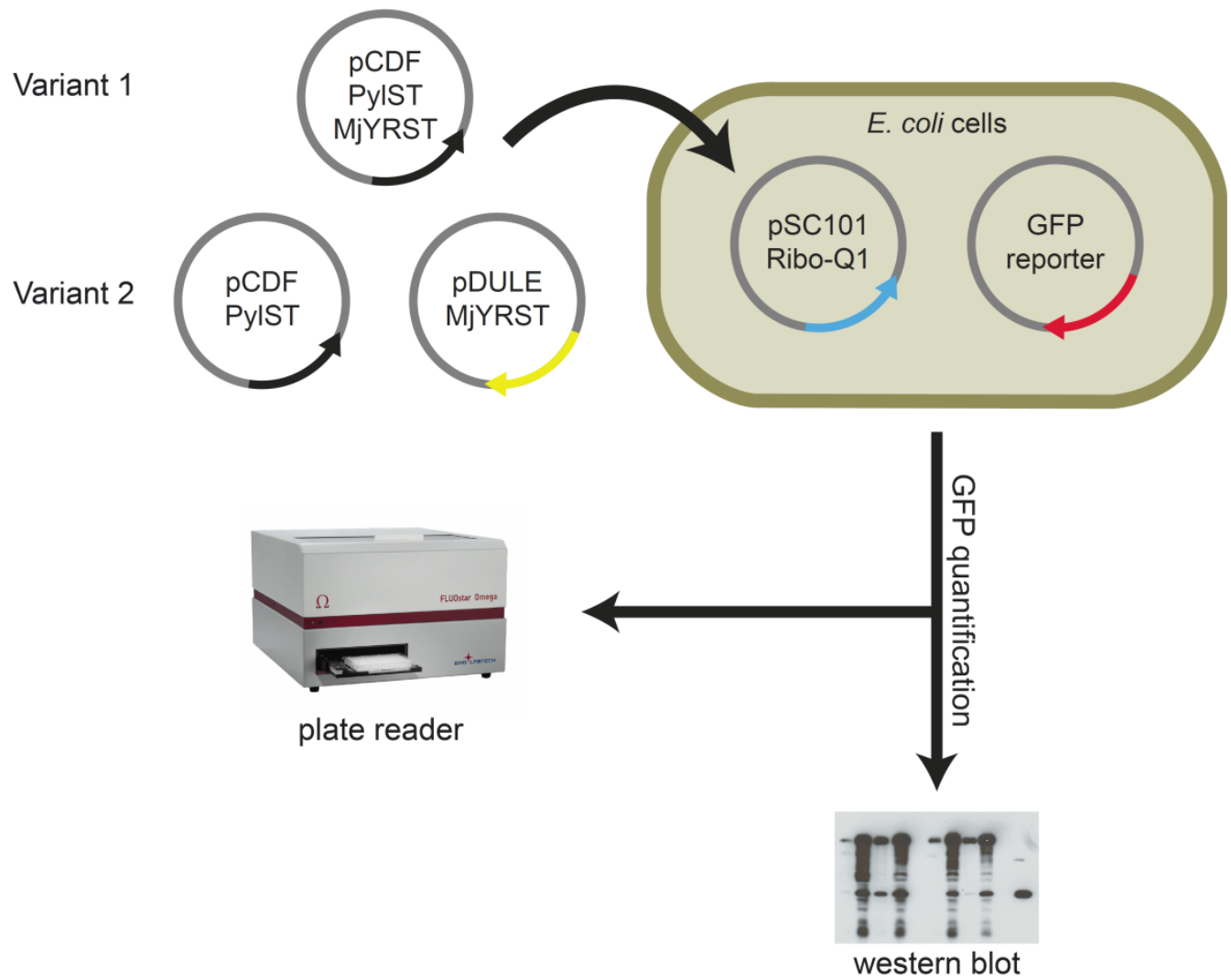

Figure 3.4: Schematic view of the GFP reporter assay.

Electro competent $E$. coli $\mathrm{DH} 10 \mathrm{~B}$ cells were prepared that already bear a plasmid for the orthogonal ribosome ribo-Q1 ${ }^{[78]}$ (pCLA96; Table 8.1) and one out of four GFP reporter plasmids (pCLA105 to pCLA108; Ch. 4.4.3). These cells were transformed with two different sets of plasmids, either Variant 1 or Variant 2. Variant 1 comprised a pCDF Duet-1 (Novagen; pCLA91) based vector, which holds the genes for the tRNA/aaRS pairs PyIT/PyIS (PyIST) as well as MjYT_UCCU/MjYRS_AGGA (MJYRST) (pCLA95). Variant 2 included the same tRNA/aaRS pairs as Variant 1 , but split on two plasmids - pCDF PyIST ${ }^{[78]}$ (pCLA97) and pDULE MjYRST (this version decodes for UCCU and is based on the CUA version ${ }^{[68,83]}$; pCLA98). The suppression efficiency of amber and/or frameshift codons was measured by the amount of GFP produced. GFP was either detected using a plate reader (Ch. 3.2.2.12) or by western blot (Ch. 3.2.2.4). The better a plasmid combination worked, the more GFP fluorescence could be detected. Resistance genes on plasmids are indicated by the arrows: black =Sm; blue $=$ Kan; red $=$ Amp; yellow $=$ Tet 


\subsubsection{Barnase Reporter Assay}

The barnase assay is used to delete non-orthogonal synthetases that charge their corresponding tRNAs with natural amino acids or non-orthogonal tRNAs that are aminoacylated by endogenous synthetases. This negative selection is based on suppression of amber nonsense mutations in the barnase gene as described by Wang and Schultz ${ }^{[146]}$. The barnase protein is an extracellular ribonuclease that originates from Bacillus amyloliquefaciens. Without its inhibitor, barstar, it is lethal to cells ${ }^{[46]}$. That means, synthetases that are using natural amino acids in the absence of the UAA will finally suppress the amber codons in the barnase gene and mediate the cell death. Cells having synthetases that are still orthogonal and only using UAAs will be selected for (Figure 3.5).

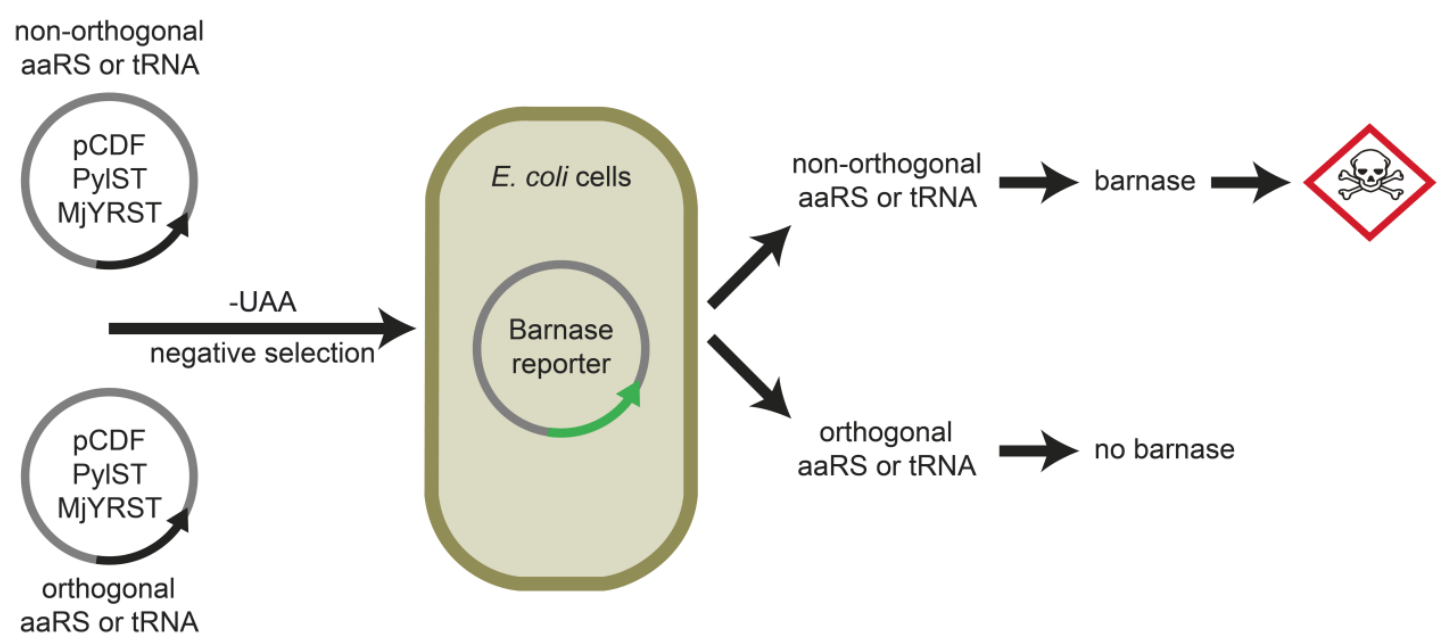

Figure 3.5: Schematic view of the barnase reporter assay.

Electro competent E. coli DH10B cells containing the barnase reporter plasmid (pCLA114; Table 8.1) were transformed with pCDF four library plasmids. The mixture of the library plasmids comprised undesired mutated aaRS or tRNAs that are no longer orthogonal and desired orthogonal aaRS and tRNAs. Cells were plated on agar plates with appropriate antibiotics in the absence and presence of $0.002 \%$ arabinose, to induce the expression of the barnase gene, and $1 \mathrm{mM} \mathrm{Bock}$, as a negative control. If no UAA was added but the barnase expression induced by arabinose, cells with mutated aaRS or tRNAs are supposed to die because of the usage of natural amino acids or the aminoacylation by endogenous synthetases and thereby the suppression of the amber codons within the barnase gene. Thus, only cells with orthogonal aaRS or tRNAs should survive due to no expression of barnase. Resistance genes on plasmids are indicated by the arrows: black $=\mathrm{Sm}$; green $=\mathrm{Cm}$. 


\section{Results}

\subsection{Abundance And Activity of The aaRS/tRNA Pairs PylS/PylT And MjYRS/MjYT}

The expansion of the genetic code allowed for the site-specific incorporation of synthetic, unnatural amino acids (UAAs) into proteins comprising a diversity of functionalities like posttranslational modifications, UV-activatable crosslinkers or photo-reactive moieties ${ }^{[42]}$. These modified proteins have been used to investigate biological questions, for example, how acetylated lysines impact chromatin dynamics ${ }^{[97]}$ and gene expression ${ }^{[147]}$. The UVactivatable crosslinker $p$-benzoyl-L-phenylalanine helped to understand the complicated but important biological process of chromosome condensation in mitosis ${ }^{[86]}$.

Although this technique is a powerful tool for investigating protein function, the incorporation efficiency of UAAs is limited. Several approaches aimed at optimization of the existing systems by addressing suppressor tRNA competition with release factor 1 (RF1) for binding to amber codons in the A-site. This represents one of the major reasons for truncated proteins and is accentuated for protein expression aimed at incorporating multiple identical UAAs. Johnson and colleagues overcame this issue by knocking out the essential RF1 and simultaneously "fixing" RF2 ${ }^{[57]}$. The workgroups of Church and Isaacs reassigned UAG as a sense codon by genome wide replacing with the synonymous UAA codon $^{[64]}$ using multiplex automated genome engineering $(\mathrm{MAGE})^{[63]}$, permitting the deletion of RF1 ${ }^{[65]}$.

Other methods included the use of orthogonal ribosomes, which allowed for the formation of a parallel, and independent, translational machinery that was evolved to decode amber and quadruplet codons with a significantly enhanced efficiency ${ }^{[67,78]}$. Furthermore, a variety of strategies to optimize aaRS and tRNA levels have been pursued that led to improved yields of full-length protein ${ }^{[51,69,72]}$.

Nevertheless, none of the approaches performed to optimize the incorporation efficiency of UAAs into proteins provided a systematic study on the correlation of aaRS and tRNA expression levels, including the loading status of the tRNA. Thus, only little insight is given into which of these components is the actual limiting factor. Here, we describe the establishment of assays for the detection of the abundance and activity of each component (PyIS/PyIT and MjYRS/MjYT) individually and of both tRNA/aaRS pairs in combination. This 
This alignment, as well as alignments with PylS from other organisms (data not shown), revealed a flexible linker region between the tRNA binding domain in the $\mathrm{N}$-terminus and the catalytic domain of the C-terminus ${ }^{[150]}$. This linker region started around L118 and ended at E183 (according to the sequence of M. barkeri). This part of the protein was considered potentially less sensitive for mutagenesis. With the additional help of the crystal structure from M. mazei (Figure 4.2) we chose two positions for the incorporation of an internal $\mathrm{His}_{6}$-tag, flanked by glycines. One tag was directed into the "gap" between S144 and S145, evoked by the lack of 34 amino acids in the sequence of $M$. barkeri compared to M. mazei (Figure 4.1; pCLA9) and the other one was guided into a loop which projects out of the structure (Figure 4.2; pCLA10).

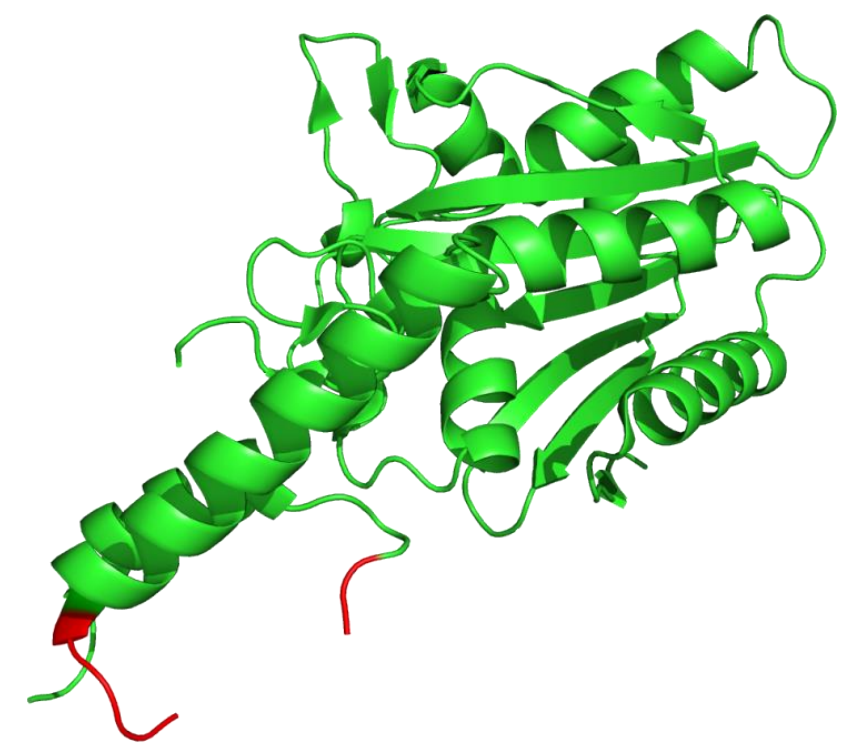

Figure 4.2: Crystal structure of the catalytic domain of PylS from M. mazei.

This figure was made with PyMOL ${ }^{[151]}$ using pdb file $2 Z_{1}{ }^{[152]}$ from the RSCB PDB database ${ }^{[153]}$. The highlighted red residues indicate the loop D206 to F216 (D171 to F181 in the M. barkeri sequence) in the linker region between $\mathrm{N}$ - and $\mathrm{C}$-terminus. This loop was chosen to incorporate a $\mathrm{His}_{6}$-tag because it sticks out of the structure away from the catalytic core.

To investigate if the integration of internal $\mathrm{His}_{6}$-tags had an influence on the catalytic activity of the enzyme, a chloramphenicol reporter assay (Ch. 3.2.4.1) was performed. Hence, E. coli cells were co-transformed with the pREP PyIT reporter (pCLA4) and pBK plasmids containing PylS genes with internal tags (pCLA9 and pCLA10), without a $\mathrm{His}_{6}$-tag (pCLA1) and with a C-terminal tag (pCLA8) (Figure 4.3). The assay showed that cells with wild type (WT) PyIS and PylS with a His ${ }_{6}$-tag introduced into the "gap" between S144 and S145 exhibit the same growth behavior and were resistant to a $\mathrm{Cm}$ concentration of approximately $400 \mu \mathrm{g} / \mathrm{mL}$. Whereas cells harboring PylS with a C-terminal tag died around $75 \mu \mathrm{g} / \mathrm{mL} \mathrm{Cm}$, cells with the loop version survived $\mathrm{Cm}$ up to $150 \mu \mathrm{g} / \mathrm{mL}$. 


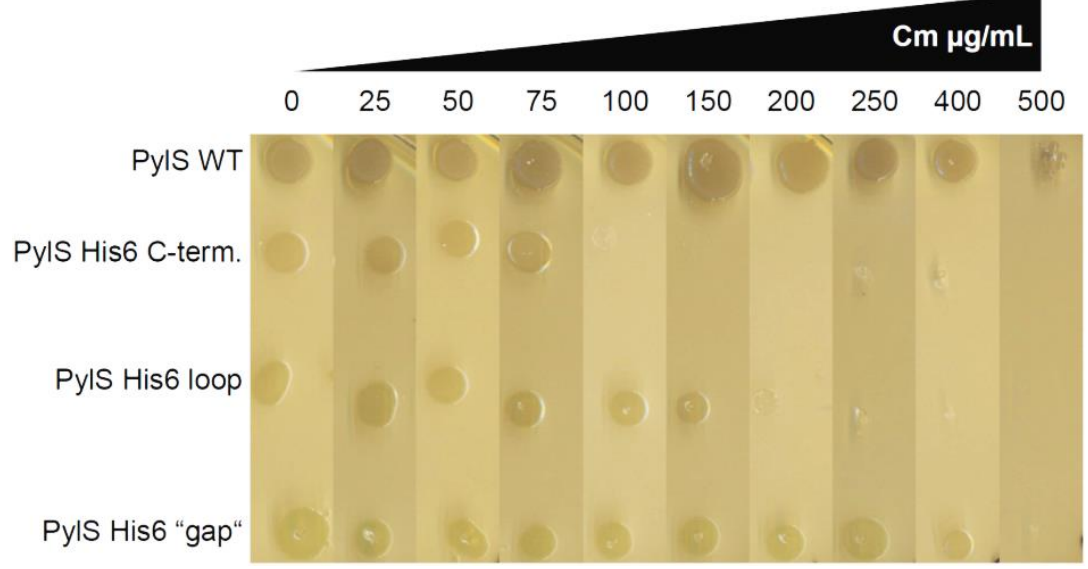

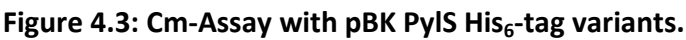

The catalytic activity of wild type (WT; pCLA1) PyIS was compared with different $\mathrm{His}_{6}$-tagged PylS variants (pCLA8 to pCLA10). Therefore a chloramphenicol reporter assay (Ch. 3.2.4.1) was performed and cells were plated on agar plates containing Kan, Tet, increasing $\mathrm{Cm}$ and $1 \mathrm{mM}$ BocK. PylS with a tag introduced into the "gap" between S144 and S145 (pCLA9) showed an activity comparable to the WT (pCLA1)since cells were able to grow up to a $\mathrm{Cm}$ concentration of $400 \mu \mathrm{g} / \mathrm{mL}$. A His ${ }_{6}$-tag at the C-terminus (C-term; pCLA8) allowed the cells to survive a $\mathrm{Cm}$ concentration of $75 \mu \mathrm{g} / \mathrm{mL}$, only. The loop variant (pCLA10) let the cells grow up to $150 \mu \mathrm{g} / \mathrm{mL} \mathrm{Cm}$.

Thus, the PyIS "gap" variant (pCLA9) was the most promising mutant and needed to be tested for detectability on western blots. We performed a test expression using cells transformed with the appropriate plasmid and took samples in exponential growth phase, late exponential growth phase and stationary phase (Figure 4.4).

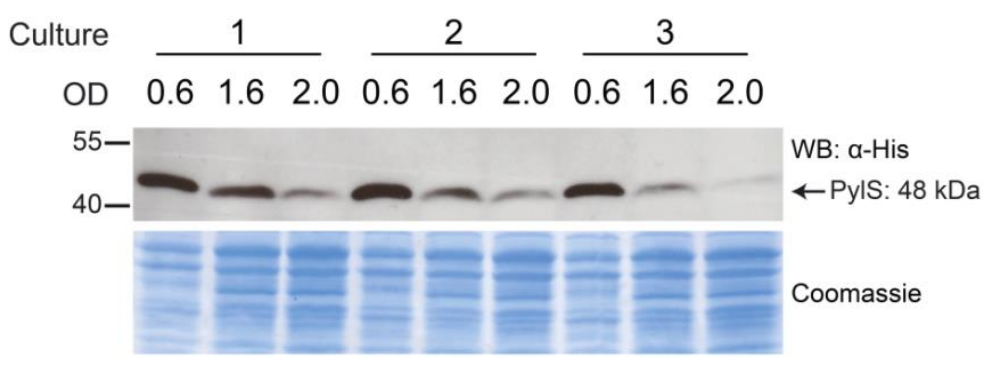

Figure 4.4: Detection of PylS His $_{6}$-tag ("gap") via western blot.

Three individual cultures with cells harboring plasmid pCLA9 were grown. Samples were taken and normalized to $\mathrm{OD}_{600}=1.5$ in exponential growth phase $\left(\mathrm{OD}_{600}=0.6\right)$, late exponential growth phase $\left(\mathrm{OD}_{600}=1.6\right)$ and stationary phase $\left(\mathrm{OD}_{600}=2.0\right)$. Whole cell extracts were separated with SDS PAGE (Ch. 3.2.2.3) and blotted onto a PVDF membrane (Ch. 3.2.2.4). Anti-His-antibody was used as primary antibody.

This position allowed for the detection of the $\mathrm{His}_{6}$-tag and thereby the PylS via western blot. As depicted in Figure 4.4, a decrease of the signal at late exponential growth phase and again at stationary phase could be observed, although the loading control (Coomassie stain of the gel) showed even a slight increase in total protein yield. Due to the results from the activity test and from western blot we decided to use the PyIS "gap" variant for further experiments. 
Due to the fact that no crystal structures of $M$. barkeri PyIS are currently available, the only structural insights come from that of the $M$. mazei homologue. This structure only reveals the $\mathrm{C}$-terminal domain ${ }^{[154]}$ because the highly basic, yet hydrophobic, $\mathrm{N}$-terminal domain was unstable and inadequate for crystallization trials ${ }^{[150]}$. Additionally, the $\mathrm{N}$-terminus was known to be essential for in vivo activity but dispensable in vitro ${ }^{[155]}$ and its overall function, binding of PyIT, was quite recently found by Jiang and Krzycki ${ }^{[150]}$. As mentioned above, the linker between the $\mathrm{N}$ - and $\mathrm{C}$-terminus varies in sequence and length ${ }^{[155]}$. Furthermore, it has a high content of prolines, which are responsible for the instabilities due to the inability to form hydrogen bonds and the introductions of bends, or kinks, that disrupt helices or sheets. In the case of the M. barkeri PyIS, the 66 AAs of the linker region include eight prolines, which correlate to circa $12 \%$. In summary, the aaRS PyIS exhibits several regions that destabilize the protein and thus potentially decrease the activity.

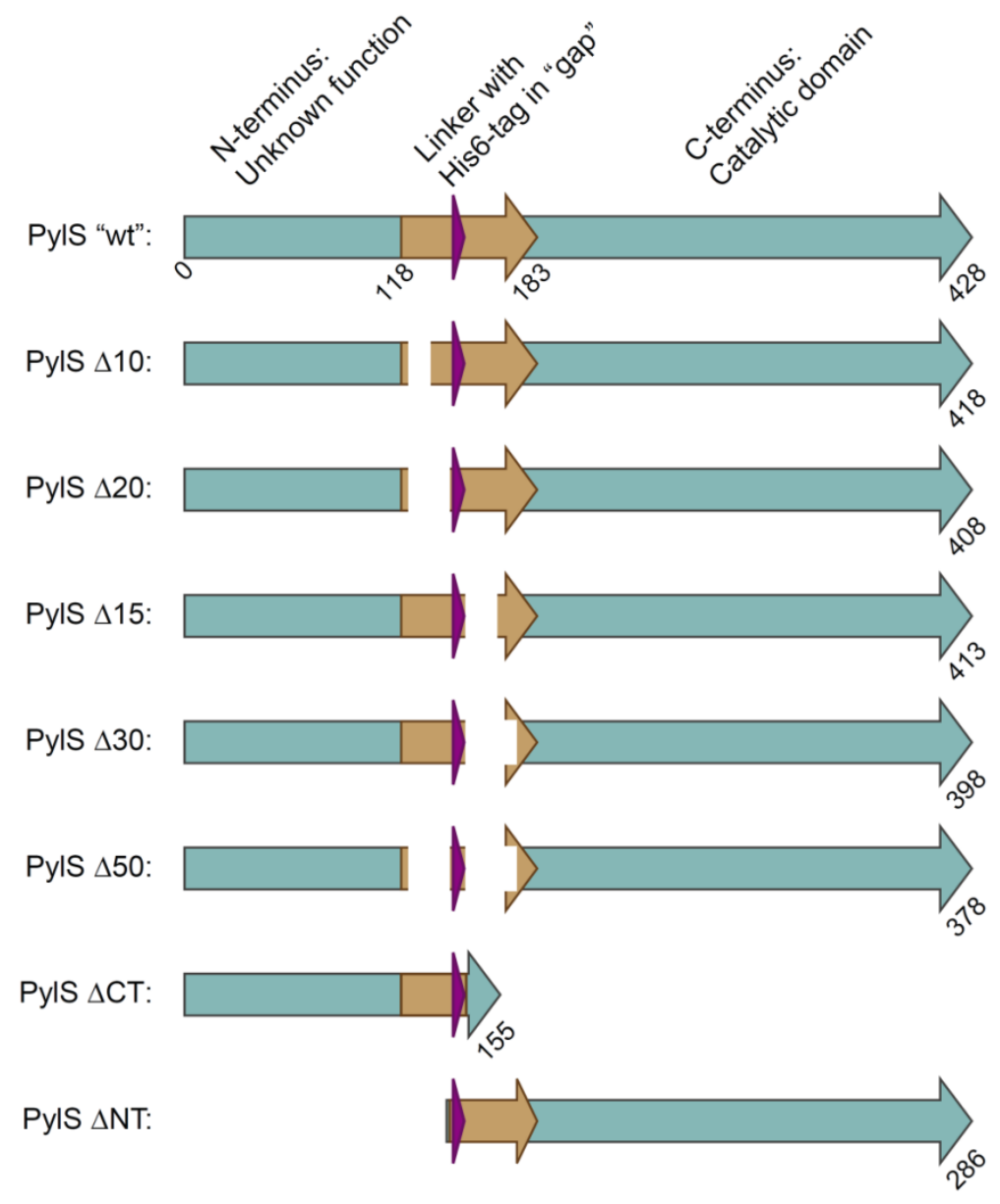

Figure 4.5: Truncations in the PyIS "gap" ("wt") variant to investigate the effect on the suppression efficiency. Truncations $(\Delta$; the number specifies the quantity of AAs deleted; $C T=C$-terminus; $N T=N$-terminus) are indicated by the white spaces and were made via QC on PCLA9 resulting in plasmids PCLA11 to pCLA17. The protein PylS is pictured by the turquois arrows with numbers indicating the length in AAs below. The linker region is highlighted by the brown arrow and the internal $\mathrm{His}_{6}$-tag is accentuated by the violet arrowhead. 
In order to determine if these parts of the protein are dispensable and thereby enhance the suppression efficiency, we produced truncations of different lengths inside the linker of the PylS "gap" variant (pCLA9). All of these truncations surrounded the internal His 6 -tag (Figure 4.5). In addition, the complete $\mathrm{N}$ - and $\mathrm{C}$-terminus were deleted to investigate the effect on the suppression efficiency as well.

To find out whether these truncations influence the activity and thereby the suppression efficiency of PyIS a chloramphenicol reporter assay was performed (Figure 4.6). Therefore, competent $E$. coli cells harboring the pREP PyIT reporter (pCLA4) were transformed with the truncation plasmids (pCLA11 to pCLA17) and compared to PyIS "gap" without any changes ( $\mathrm{PCLA9}$ ) as a reference.

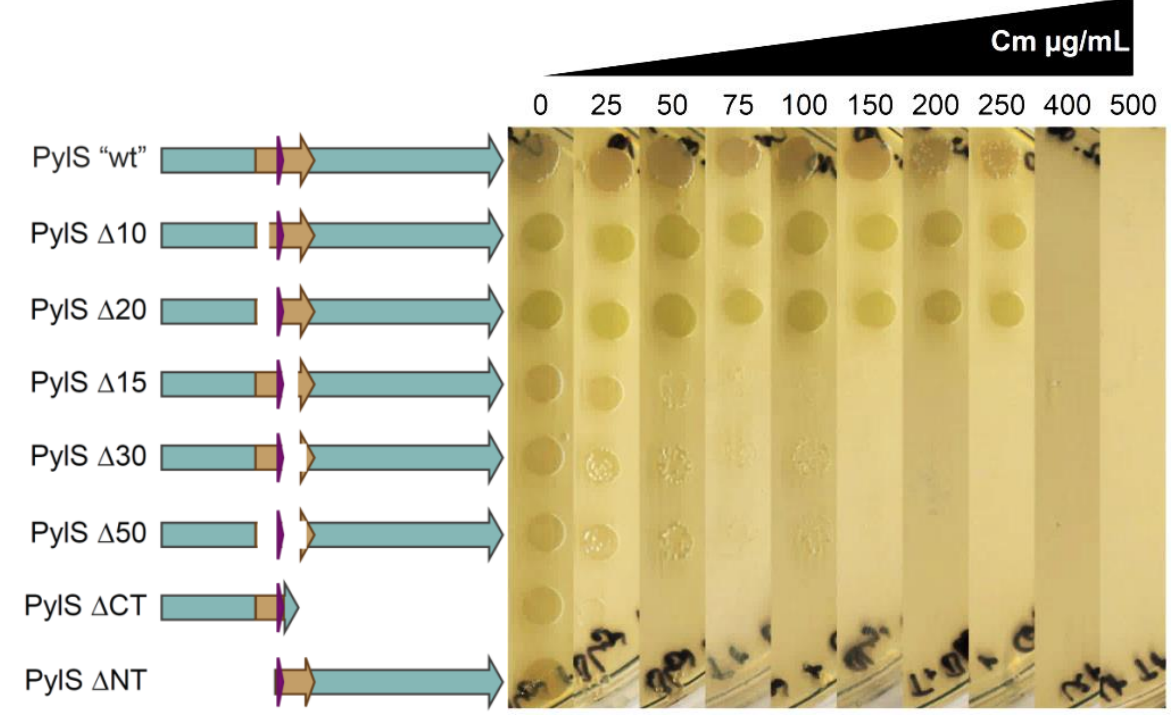

Figure 4.6: Cm-Assay with truncation variants of PylS "gap" (“wt”).

The catalytic activity of "wt" PyIS "gap" variant (pCLA9) was compared with different truncations made in the linker region between $\mathrm{N}$ - and C-terminus ( $\mathrm{pCLA11}$ to $\mathrm{pCLA17}$; Figure 4.5). Therefore a chloramphenicol reporter assay (Ch. 3.2.4.1) was performed and cells were plated on agar plates containing Kan, Tet, increasing $\mathrm{Cm}$ and $1 \mathrm{mM}$ Bock. Deletions at the beginning of the linker, $\Delta 10$ and $\Delta 20$, did not influence the suppression efficiency negatively but cells were also not able to survive higher $\mathrm{Cm}$ concentrations than cells with the "wt" variant. All three versions let cells grew to a $\mathrm{Cm}$ concentration of $250 \mathrm{\mu g} / \mathrm{mL}$. Cells with the other truncated PylS variants started dying already between $\mathrm{Cm} 25$ and $50 \mu \mathrm{g} / \mathrm{mL}$. Whereas the smaller truncations in the linker regions still allowed survival of a few cells up to $\mathrm{Cm} 100 \mu \mathrm{g} / \mathrm{mL}$, especially the deletion of the C-terminus prevented growth on plates with $\mathrm{Cm}$ higher than $25 \mu \mathrm{g} / \mathrm{mL}$.

We observed that the $\mathrm{N}$-terminal linker truncations, $\Delta 10$ and $\Delta 20$, revealed the same growth behavior as the "wild type" PylS. The suppression efficiency was neither decreased nor significantly increased. All three versions allowed cell growth to a $\mathrm{Cm}$ concentration of $250 \mu \mathrm{g} / \mathrm{mL}$. Deletions in the C-terminal linker region, as well as the deletion of the whole $\mathrm{N}$ and C-terminus, caused the cells to die between $\mathrm{Cm} 25$ and $50 \mu \mathrm{g} / \mathrm{mL}$. The removal of the C-terminus caused a severe effect because no growth on agar plates with $\mathrm{Cm}$ higher than 
$25 \mu \mathrm{g} / \mathrm{mL}$ was noticed. The truncations $\Delta 15, \Delta 30, \Delta 50$ and $\Delta \mathrm{NT}$ permitted very weak growth up to $100 \mu \mathrm{g} / \mathrm{mL}$ chloramphenicol.

Since truncations of the PyIS "gap" variant (pCLA9) did not result in a significant improvement of the suppression efficiency we used the "wt" version of this variant in further experiments.

\subsubsection{PylT of Methanosarcina barkeri}

For the optimization of the aaRS PyIS (Ch. 4.1.1) it was also necessary to analyze the cognate tRNA PyIT for its abundance and loading status. We chose to approach this assay using Northern blotting (Ch. 3.2.3.15) techniques and therefore hybridization probes had to be designed. As described in Ch. 3.2.3.15, we used DNA oligonucleotides labeled with Cy3, Cy5 or DIG, complementary to the anticodon stem and loop domain of the desired tRNA target. The oligos C25 and C26 (Table 8.2) were designed to anneal to the anticodon loop of PylT as shown in Figure 4.7.

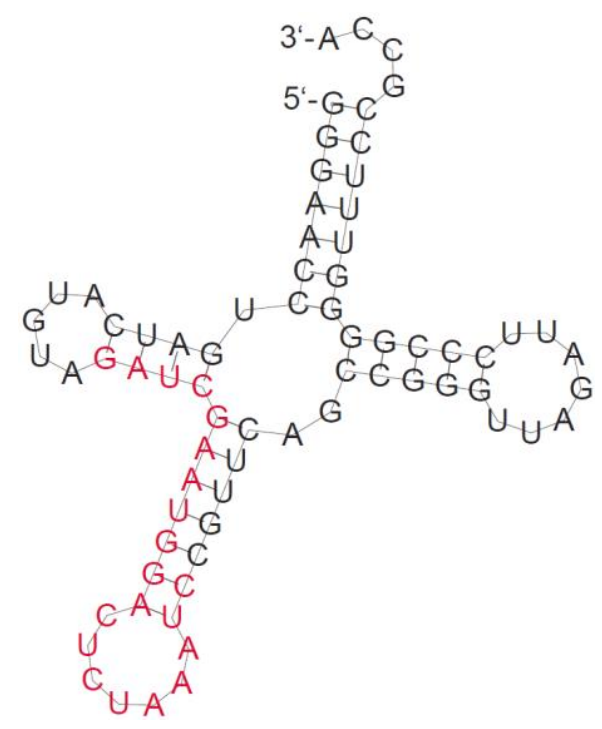

Figure 4.7: Predicted secondary structure of PylT.

The structure was obtained from ViennaRNA Web Services ${ }^{[156]}$. The region to which the probes C25 and C26 bind is highlighted in red, representing the anticodon stem and a section of the D-loop.

Initial trials for the detection of PylT were carried out with in vitro synthesized targets. These were the DNA oligonucleotide C27 that represented the bases 13-42 (of 72) from PylT and a RNA transcript of PyIT (T7 PyIT; Ch. 3.2.3.16). Both targets could be detected with signal intensities depending on the concentration of the target as depicted in Figure 4.8-A. We first approached this detection using fluorophore labeled probes (Cy3 and Cy5) 
for hybridization. We then altered our approach to include DIG labeled probes due to increased sensitivity, as shown for T7RP transcribed E. coli Ser-tRNA-1 (T7 SerT; Figure 4.8-B).

A)

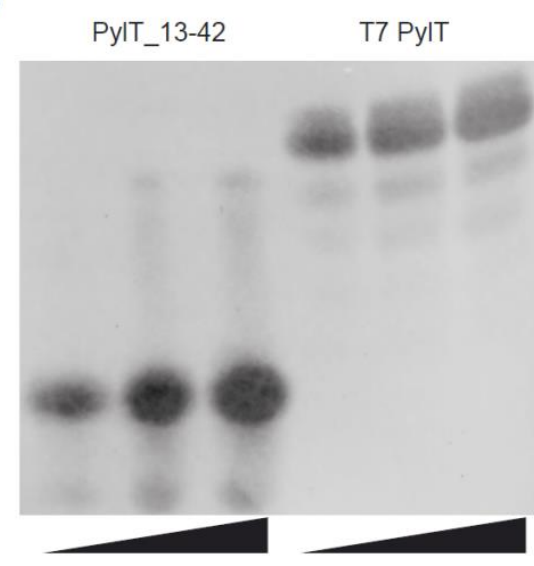

B)

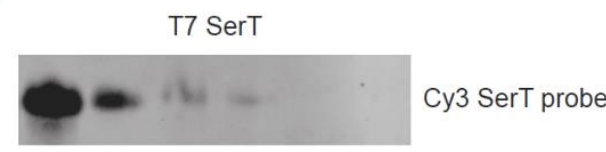

Cy3 PylT probe

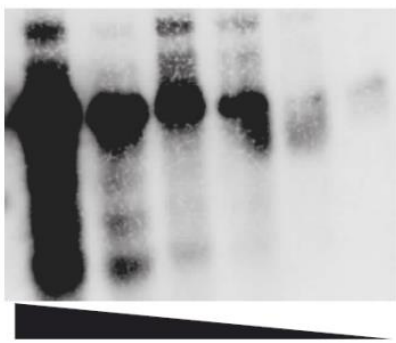

Dig SerT probe

Figure 4.8: Establishing northern blots to detect specific tRNAs.

A) To verify the ability of the hybridization probes C25 and C26 to bind to the specific target tRNA PylT, artificial samples were separated with acid urea PAGE and subsequently blotted (Ch. 3.2.3.15). DNA oligonucleotide C27 (PylT_13-42) was loaded with a total amount of 2.3, 4.6 and $6.9 \mu \mathrm{g}$, respectively (left to right, indicated by the black triangle). In contrast 6.0, 12.0 and $18.0 \mu \mathrm{g}$ of T7 PylT were loaded. A Cy3 labeled PyIT probe (C25) was used for hybridization and visualized by a Typhoon imager (Excitation: 532 nm; Filter: 580 BP 30; PMT 400; Resolution: $50 \mu \mathrm{m}$ per pixel). B) Comparison of a Cy3 labeled probe (C32; upper panel) against E. coli Ser-tRNA-1 to a DIG labeled probe (C33; lower panel). A total amount of 13.3, 1.33, 0.48, 0.24, 0.048 and $0.024 \mu \mathrm{g}$ T7 SerT was loaded, respectively (right to left). The Cy3 probe was visualized by a Typhoon imager (Excitation: $532 \mathrm{~nm}$; Filter: 580 BP 30; PMT 460; Resolution: $200 \mu \mathrm{m}$ per pixel) and the DIG probe by decorating with an anti-DIG-APantibody followed by a chemiluminescence reaction using CDP-Star as described in Ch. 3.2.3.15.

The next step was to detect the PyIT in total RNA extracts from E. coli cells. Hence, different cell cultures harboring plasmids for either PyIS only or PyIS and PyIT (pCLA1 and pCLA4) were grown in the presence, or absence, of Bock. Total RNA was isolated (Ch. 3.2.3.14) and separated with acid urea PAGE. Several standards (Ch. 3.1.4) were used to estimate the size of the isolated RNA compared to the positive control T7 PyIT, described above (Figure 4.9-A). The T7 PyIT gave a signal around $70 \mathrm{nt}$ which fits to the actual size of the tRNA with $72 \mathrm{nt}$. Total RNA extracts from cells yielded a smear, as expected, due to the many distinct RNA fragments with different lengths, like rRNAs, mRNAs, tRNAs, riboswitches etc. Subsequent northern blots of separated RNA revealed positive signals for PyIT only if the appropriate plasmid was present in the cells (Figure 4.9-B). Decreasing the concentration of loaded RNA samples sufficiently resulted in the emergence of a second band with different migratory behavior. This second band only appeared if RNA was isolated from cells grown in medium supplemented with Bock (Figure 4.9-C). The appearance of the second signal, depending on the addition of the amino acid, suggested that the upper band represented the aminoacylated form of PylT and the lower one the uncharged form. 
A)

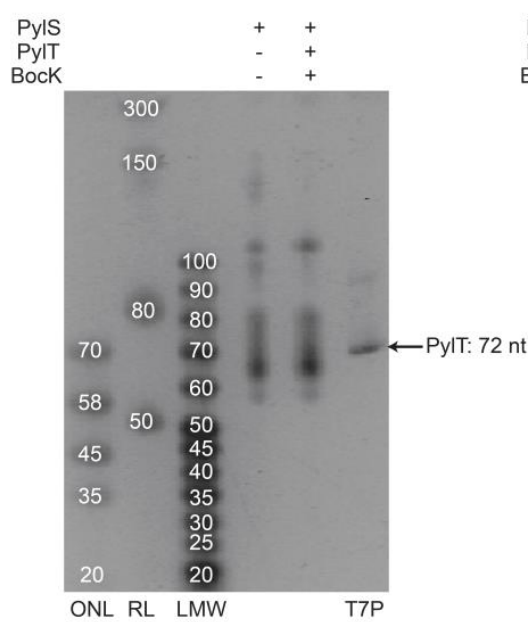

B)

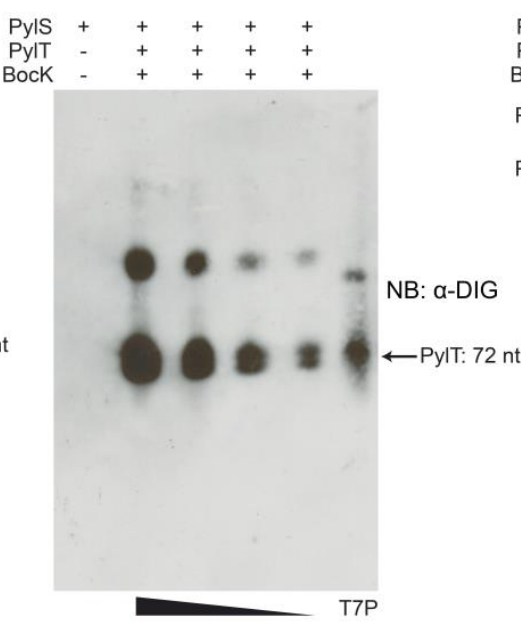

C)

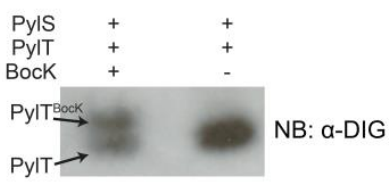

Figure 4.9: Detection of PylT in total RNA extracts.

A) Acid urea PAGE for the size estimation of isolated RNA from cell cultures harboring plasmids for PyIS ( $p C L A 1$; $1.4 \mu \mathrm{g}$ loaded onto gel) and PyIS/PylT (pCLA1 and pCLA4; $1.45 \mu \mathrm{g}$ loaded). The latter one was grown in LB medium supplemented with $1 \mathrm{mM}$ Bock. T7 PyIT (T7P) with a size of $72 \mathrm{nt}$ served as a positive control $(0.5 \mu \mathrm{g}$ loaded). Three different size standards consisting of DNA and RNA nucleotides were used to consider different running behavior. ONL = Oligonucleotide Ladder; RL = Low Range ssRNA Ladder; LMW = Low Molecular Weight Marker. The gel was soaked in a "gel red" bath $\left(1: 10,000\right.$ in $\left.\mathrm{H}_{2} \mathrm{O}(\mathrm{v} / \mathrm{v})\right)$ and separated DNA/RNA bands were visualized by UV light using a gel documentation machine. B) Northern blot of samples described in A). A total amount of $2.8 \mu \mathrm{g}$ RNA from cells with PylS only was loaded. In contrast 1.45, 0.7, 0.35 and $0.17 \mu \mathrm{g}$ RNA from cells with PylS and PyIT (+ $1 \mathrm{mM}$ BocK) were loaded (left to right, indicated by the black triangle). $0.03 \mu \mathrm{g}$ of the positive control T7 PyIT were used. The DIG labeled probe C26 was utilized for hybridization and chemiluminescence signals recorded as described in Ch. 3.2.3.15. C) Northern blot for the comparison of cell cultures grown in the presence and absence of $1 \mathrm{mM}$ Bock in the medium. Approximately $0.045 \mu \mathrm{g}$ RNA were loaded for each sample. Signals were detected as in B). The upper band $\left(\mathrm{PyIT}^{\mathrm{Bock}}\right.$ ) represents the aminoacylated form of PyIT, whereas the lower band (PyIT) depicts the uncharged form.

\subsubsection{MjYRS of Methanocaldococcus jannaschii}

Analogous to PyIS (Ch. 4.1.1) no antibodies against the tyrosine synthetase (MjYRS) of the archaeon Methanocaldococcus jannaschii (M. jannaschii) were available. Thus, we again took the small $\mathrm{His}_{6}$-tag as an alternative and cloned it into the N-terminus (pCLA18). We observed again that, similar to the modifications on PyIS, no signals on a western blot against the $\mathrm{His}_{6}$-tag were obtained. Shifting of the tag from $\mathrm{N}$ - to C-terminus (pCLA19), we again observed no signal from a western blot (Figure 4.12). Hence, comparable to PyIS, we chose an internal position for the $\mathrm{His}_{6}$-tag based on the crystal structure depicted in Figure 4.10. 


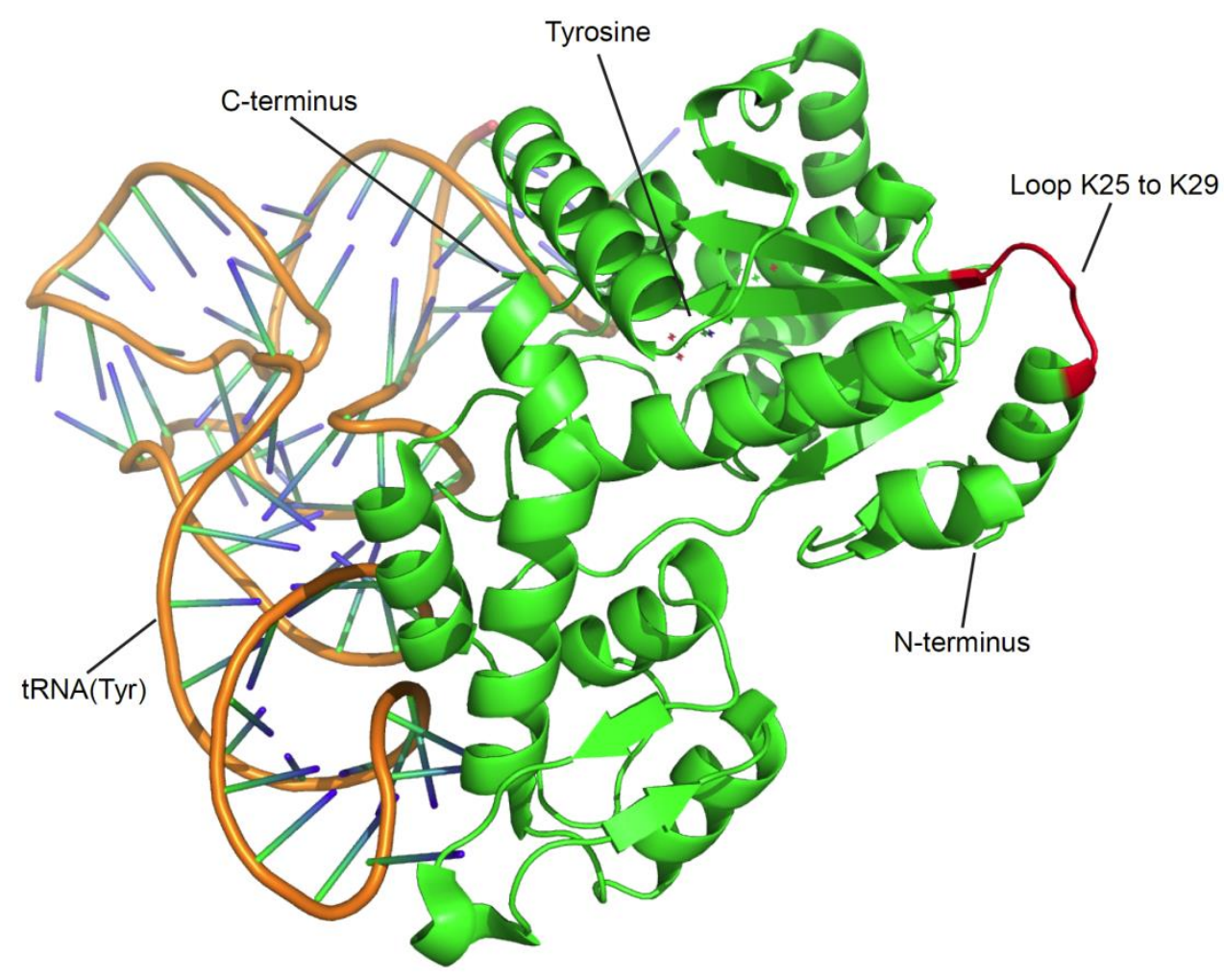

Figure 4.10: Crystal structure of tyrosyl-tRNA synthetase from M. jannaschii complexed with tRNA(Tyr) and L-tyrosine.

This figure was made with PyMOL ${ }^{[151]}$ using pdb file $1 J 1 \mathrm{U}^{[73]}$ from the RSCB PDB database ${ }^{[153]}$. The highlighted red residues indicate the loop $\mathrm{K} 25$ to $\mathrm{K} 29$. This loop was chosen to incorporate a $\mathrm{His}_{6}$-tag because it protrudes from the structure away from the catalytic core. The integration into this loop should not interfere with the tRNA sterically.

The $\mathrm{His}_{6}$-tag, again flanked by glycines, was incorporated into the loop K25 to K29 (pCLA20). As shown in Figure 4.10 the highlighted red loop points away from the catalytic core, indicated by the tyrosine, as well as from the corresponding tRNA. Thus, the mutation should not constrict protein activity what was verified by a chloramphenicol reporter assay (Ch. 3.2.4.1). Therefore E. coli cells were transformed with $\mathrm{pBK}$ plasmids harboring the genes for MjYRS WT, C-terminal and internal His 6 -tag (pCLA2, pCLA19 and pCLA20), respectively, together with the appropriate reporter plasmid (pCLA5).

It seemed that the incorporation of the $\mathrm{His}_{6}$-tag had no negative influence on the overall catalytic activity. Cells were able to survive all given concentrations of chloramphenicol up to $500 \mu \mathrm{g} / \mathrm{mL}$, independent of the MjYRS version (Figure 4.11). 


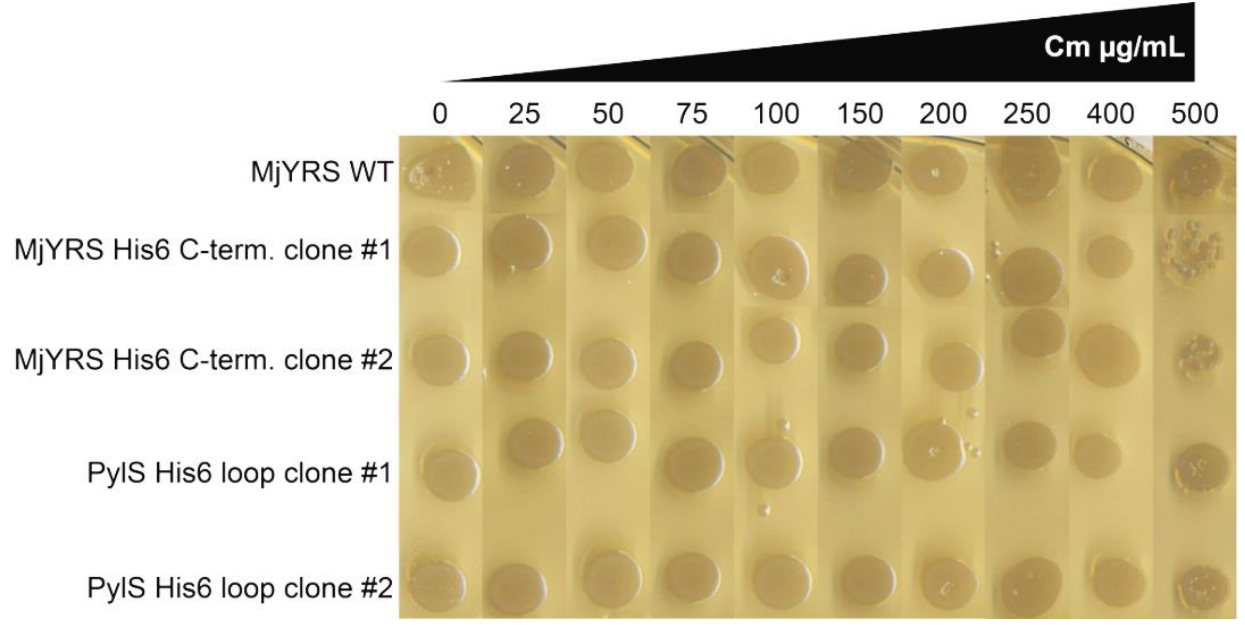

Figure 4.11: $\mathrm{Cm}$-Assay with pBK MjYRS $\mathrm{His}_{6}$-tag variants.

The catalytic activity of wild type (WT; pCLA2) MjYRS was compared with different $\mathrm{His}_{6}$-tagged MjYRS variants (pCLA19 and pCLA20). Therefore a chloramphenicol reporter assay (Ch. 3.2.4.1) was performed and cells were plated on agar plates containing Amp, Tet and increasing $\mathrm{Cm}$. The $\mathrm{His}_{6}$-tagged versions were plated in duplicates

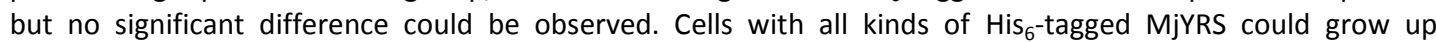
$500 \mu \mathrm{g} / \mathrm{mL} \mathrm{Cm}$. If at all, the C-terminal tagged versions show a slightly weaker growth at the highest $\mathrm{Cm}$ concentration.

The next step was to investigate if the MjYRS “loop" variant (pCLA20) could be detected via western blot in contrast to the $\mathrm{N}$ - and C-terminal tagged variants. We performed a test expression using cells transformed with the appropriate plasmids and took samples in exponential growth phase, late exponential growth phase and stationary phase (Figure 4.12).

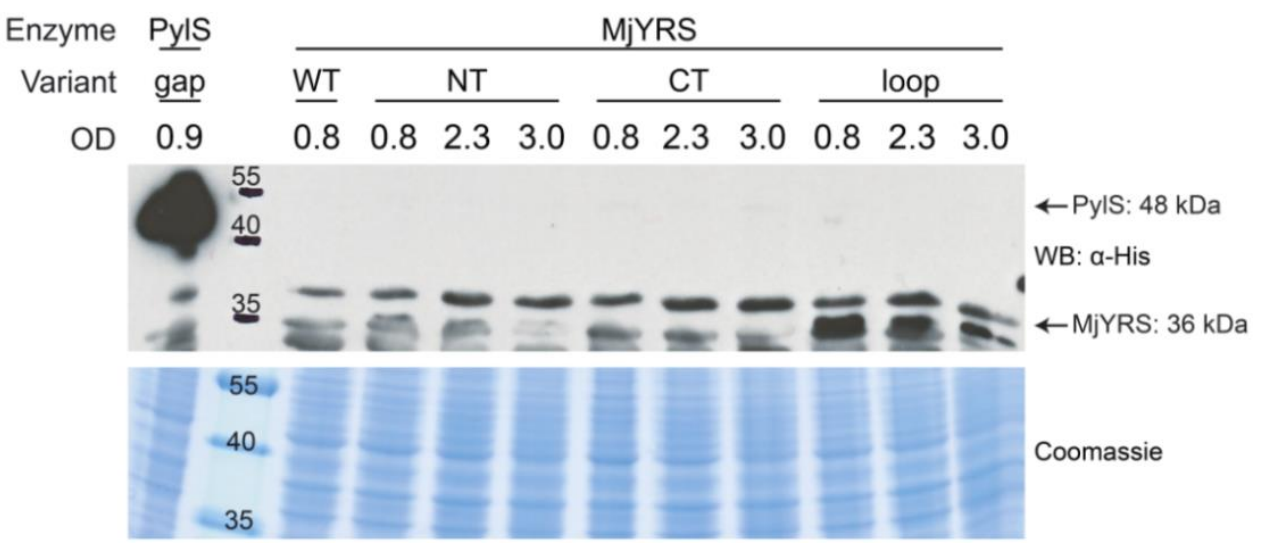

Figure 4.12: Detection of MjYRS His 6 -tag via western blot.

Four different cultures were grown with cells harboring the plasmid for WT MjYRS (pCLA2) without a His 6 -tag, Nterminal (NT) His ${ }_{6}$-tagged MjYRS (pCLA18), C-terminal tagged MjYRS (CT; pCLA19) and the "loop" variant of MjYRS (pCLA20). Samples were taken in exponential growth phase $\left(O D_{600}=0.8\right)$, late exponential growth phase $\left(O D_{600}=2.3\right)$ and stationary phase $\left(O D_{600}=3.0\right)$. Cells counts were normalized by resuspending the cell pellets in appropriate amounts of SDS sample buffer. Whole cell extracts were separated with SDS PAGE (Ch. 3.2.2.3) and blotted onto a PVDF membrane (Ch. 3.2.2.4). Anti-His-antibody was used as primary antibody. PylS "gap" from E. coli, transformed with the plasmid pCLA9, in exponential growth phase $\left(O D_{600}=0.9\right)$ served as positive control. 
In a similar manner to PyIS, as shown in Figure 4.4, only the unconventional internal $\mathrm{His}_{6}{ }^{-}$ tag gave a suitable signal on a western blot against the $\mathrm{His}_{6}$-tag. The intensity is significantly lower compared to the PylS signal, although both proteins are expressed under the same promoter. As already mentioned for PylS, the signal seemed to decrease at late exponential growth phase and again at stationary phase. The lanes for the samples taken from the culture with the N-terminal tagged MjYRS also exhibit a very weak signal of the correct size, which decreases over time and is not present in those lanes from WT or C-terminal tagged MjYRS. Considering the results from the $\mathrm{Cm}$ assay (Figure 4.11), in which no substantial difference between the MjYRS variants could be observed, this could be an indication that the proteins have a very short but distinct half-life. This could mean that there are sufficient molecules for the suppression of the amber codon but not enough left for a proper detection via western blot. The accessibility of the epitope by antibodies should not be the limiting factor because the cell lysates were prepared for western blot with denaturing conditions using SDS.

\subsubsection{MjYT of Methanocaldococcus jannaschii}

The last of the four components analyzed during this study was the corresponding tRNA of the aaRS MjYRS (Ch. 4.1.3) and its derivatives, called MjYT. MjYT, and the corresponding aaRS, were available in two different versions, where one was decoding the amber stop codon UAG (MjYT_CUA; PCLA5) and the other the frameshift codon AGGA (MjYT_UCCU; pCLA6).

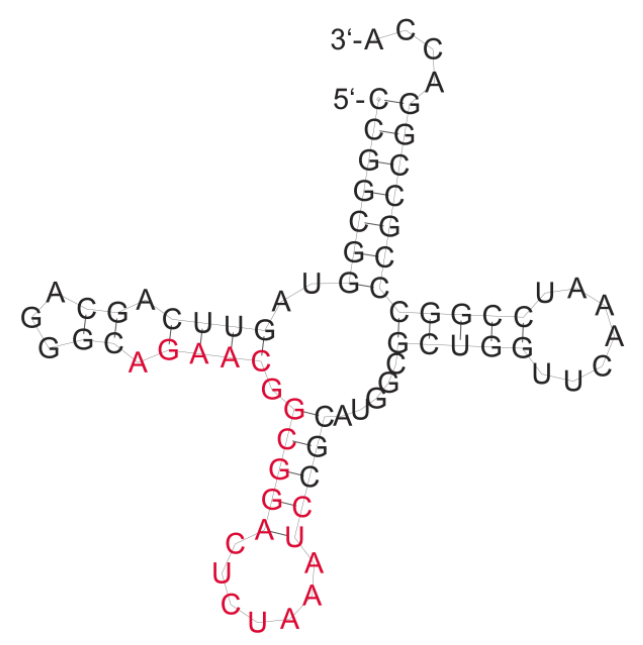

Figure 4.13: Predicted secondary structure of MjYT_CUA.

The structure was obtained from ViennaRNA Web Services ${ }^{[156]}$. The region to which the probes C40 and C41 bind, that is the anticodon stem and in parts the D-loop, is highlighted in red. 
For the detection of these tRNAs on northern blots we developed hybridization probes covering the same regions of the tRNA as shown for PyIT (Figure 4.7), namely the anticodon stem and in parts the D-loop. The oligos C40 and C41 (Table 8.2) were designed to anneal to the stop codon tRNA as shown in Figure 4.13.

Next, we extracted RNA from E. coli cells (Ch. 3.2.3.14) containing plasmids for either MjYRS_TAG only (pCLA2) or in combination with the cognate tRNA (pCLA2 and pCLA5). Additionally, RNA was isolated from cells transformed with a plasmid that held both components at once but in which the aaRS was decoding for $p$-benzoyl-L-phenylalanine ${ }^{[83]}$ (BPA; PCLA21) instead of tyrosine. Total RNA was separated with acid urea PAGE and its size was estimated by size standards (Ch. 3.1.4) and T7RP transcribed M. jannaschii TyrtRNA (T7 TyrT) as depicted in Figure 4.14-A.

\section{A)}

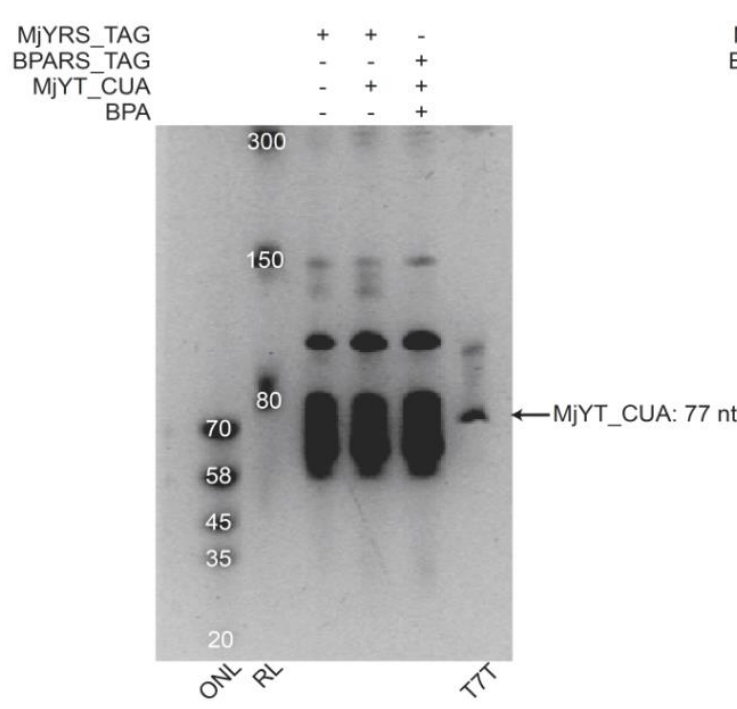

B)

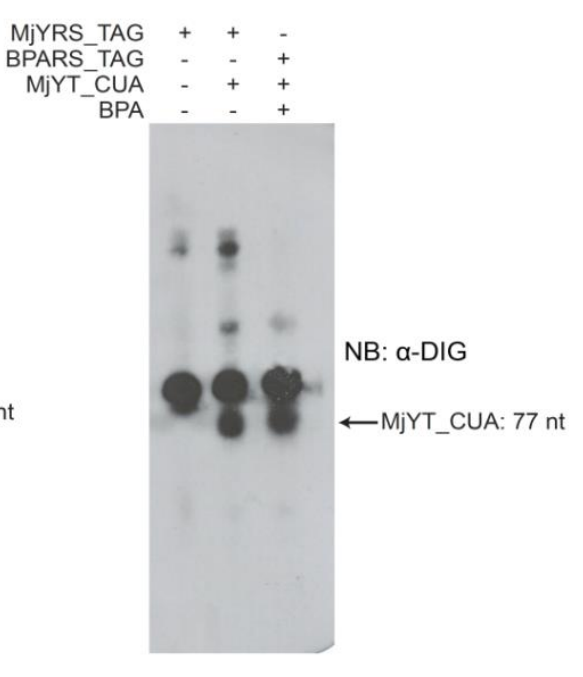

Figure 4.14: Detection of MjYT_CUA in total RNA extracts.

A) Acid urea PAGE for the size estimation of isolated RNA from cell cultures harboring plasmids for MjYRS_TAG (pCLA2; $2.8 \mu \mathrm{g}$ loaded onto gel), MjYRS_TAG/MjYT_CUA (pCLA2 and pCLA5; $2.7 \mu \mathrm{g}$ loaded) and BPARS/MjYT_CUA ( $p C L A 21 ; 3.0 \mu \mathrm{g}$ loaded). The latter one was grown in LB medium supplemented with $1 \mathrm{mM}$ BPA. T7 TyrT (T7T) with a size of $77 \mathrm{nt}$ served as a positive control $(0.8 \mu \mathrm{g}$ loaded). Two different size standards consisting of DNA and RNA nucleotides were used to consider different running behavior. ONL = Oligonucleotide Ladder; RL = Low Range ssRNA Ladder. The gel was soaked in a "gel red" bath (1:10,000 in H2O (v/v)) and separated DNA/RNA bands were visualized by UV light using a gel documentation machine. B) Northern blot of samples described in A). A total amount of $1.4 \mu \mathrm{g}$ RNA from cells with MjYRS_TAG only was loaded. In contrast 1.35 $\mu \mathrm{g}$ RNA from cells with MjYRS_TAG and MjYT_CUA and $1.5 \mu \mathrm{g}$ RNA from cells with BPARS/MjYT_CUA (+1 mM BPA) were loaded. The DIG labeled probe C41 was utilized for hybridization and chemiluminescence signals recorded as described in Ch. 3.2.3.14.

We observed a signal between 70 and $80 \mathrm{nt}$ in the lane for the T7 TyrT (Figure 4.14-A). The actual size of the tRNA is $77 \mathrm{nt}$. Total RNA extracts from cells yielded the expected smear. The northern blot of the same samples (Figure 4.14-B) showed two prominent bands. The upper one was present in all lanes and the lower one only if the cells were transformed 
with the plasmid for the tRNA. Since the lower one only appeared in samples with the tRNA and the size correlated to the signal seen in Figure 4.14-A, we assigned this band as MjYT_CUA.

The appearance of the second upper band was thought to be an unspecific binding of the probe to other RNA fragments, especially other tRNAs, due to its presence in all lanes. Thus, we blasted the sequence of the probe against the genome of E.coli (using the program BLASTN 2.2.29+ ${ }^{[157]}$, data not shown). It became apparent that the last 13 bases of the probe used for the northern blot were complementary to several kinds of $E$. coli asparagine tRNAs. Hence, new probes binding specifically to the $M$. jannaschii tRNA had to be developed and tested. Figure 4.15 shows an alignment of the two versions of MjYT, CUA and UCCU, together with those asparagine tRNAs to which the probe C41 was hybridizing. In addition to the tRNAs, all different probes tested for a specific binding to MjYT were aligned, those were MjYT_AGGA_DIG_Probe1-4 (C44 to C47). We switched to the frameshift codon version of MjYT since this tRNA was used in further experiments in combination with the UAG decoding tRNA PyIT.
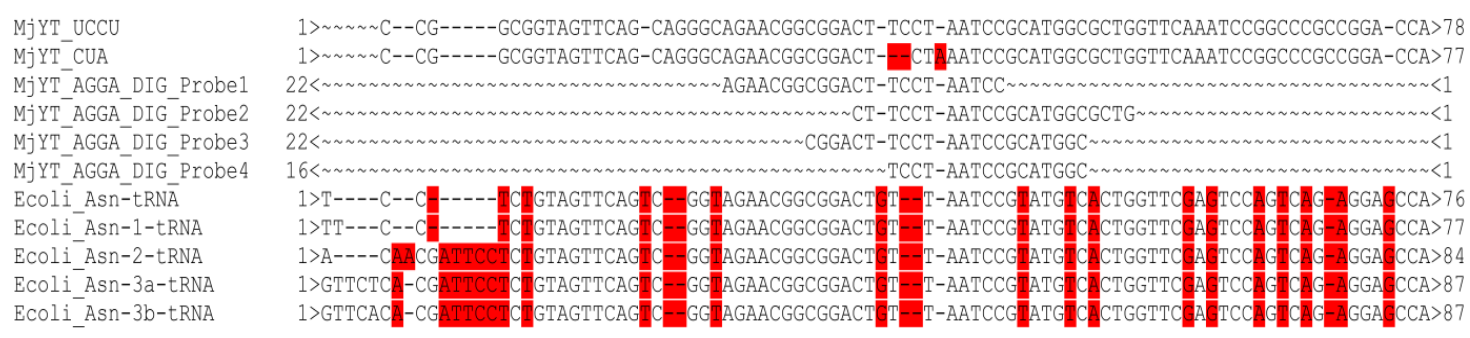

Figure 4.15: Sequence alignment of MjYT.

The DNA sequences of both MjYT versions (CUA = pCLA5 and UCCU = pCLA6) were compared to northern blot hybridization probes MjYT_AGGA_DIG_Probe1-4 (C44 to C47) and E. coli tRNAs Asn, Asn1, Asn2, Asn3a and Asn3b.

Subsequent northern blots revealed that MjYT_AGGA_DIG_Probe3 (C46; Figure 4.16-A) was the only hybridization probe that was able to bind to the tRNA MJYT_UCCU specifically without giving background signals from E. coli Asn-tRNAs (Figure 4.16-B). The other probes yielded very weak signals, no signals at all, or exhibited unspecific binding (not shown). Furthermore, Probe3 could discriminate between the two versions of MjYT, only giving signals if the frameshift variant was present in the cells. Comparable to PyIT (Figure 4.9-C) we observed two bands at different heights. Since this only happened if the corresponding aaRS was present, the upper signal was assigned as the aminoacylated form of MjYT_UCCU, again. 
A)

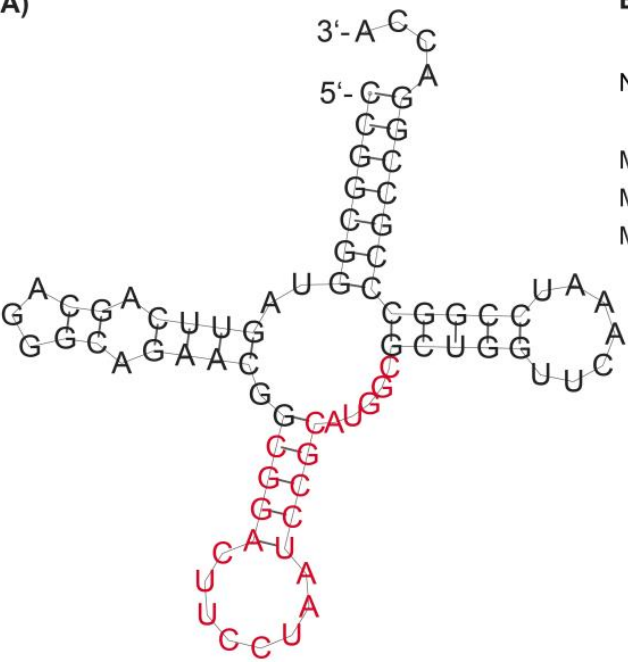

B)

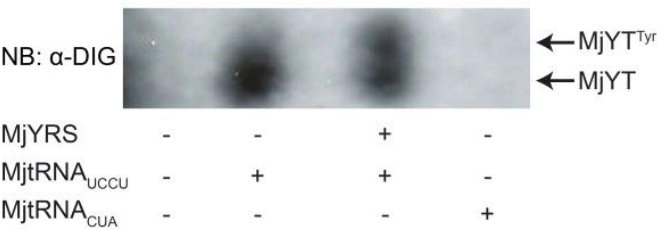

Figure 4.16: Refinement of MjYT detection.

A) Predicted secondary structure of MjYT_UCCU. The structure was obtained from ViennaRNA Web Services ${ }^{[156]}$. The region to which the probe MjYT_AGGA_DIG_Probe3 (C46) binds is highlighted in red. B) Northern blot from the refinement studies using probes MjYT_AGGA_DIG_Probe1-4 (C44 to C47). In this case only MjYT_AGGA_DIG_Probe3 (C46) is shown. Approximately $0.090 \mu \mathrm{g}$ RNA were loaded for each sample. After hybridization, chemiluminescence signals were recorded as described in Ch. 3.2.3.14. The upper band (MjYT ${ }^{\mathrm{Tyr}}$ ) represents the aminoacylated form of MjYT_UCCU, whereas the lower band (MjYT) depicts the uncharged form. 


\subsection{Promoter Libraries}

Based on the fact that both tRNA/aaRS pairs could now be detected quantitatively and their activity measured as well (Ch. 4.1), we began to optimize the suppression efficiency of both systems.

One cosideration for optimizations was already discussed in the previous chapter. The standard promoter in front of both synthetases, a glnS promoter, was not fully active during the full expression time. More precisely, we discovered a decrease of the expression levels of PyIS and MjYRS over time(Figure 4.4 and Figure 4.12). In theory, a higher expression level of a certain synthetase should lead to a higher rate of aminoacylation of the corresponding tRNA and thus, to a more efficient decoding of the appropriate codon. We believed this could be achieved by the introduction of a stronger synthetase promoter. However, a pure overexpression could also cause negative effects due to toxicity, especially if the component is not endogenous. The use of multiple elements in one cell could boost those negative effects.

Therefore, we tried to optimize the expression levels of both aaRS and tRNAs individually and in combination. For this purpose synthetic promoter libraries were used to fine tune the expression of the appropriate genes ${ }^{[158,159]}$.

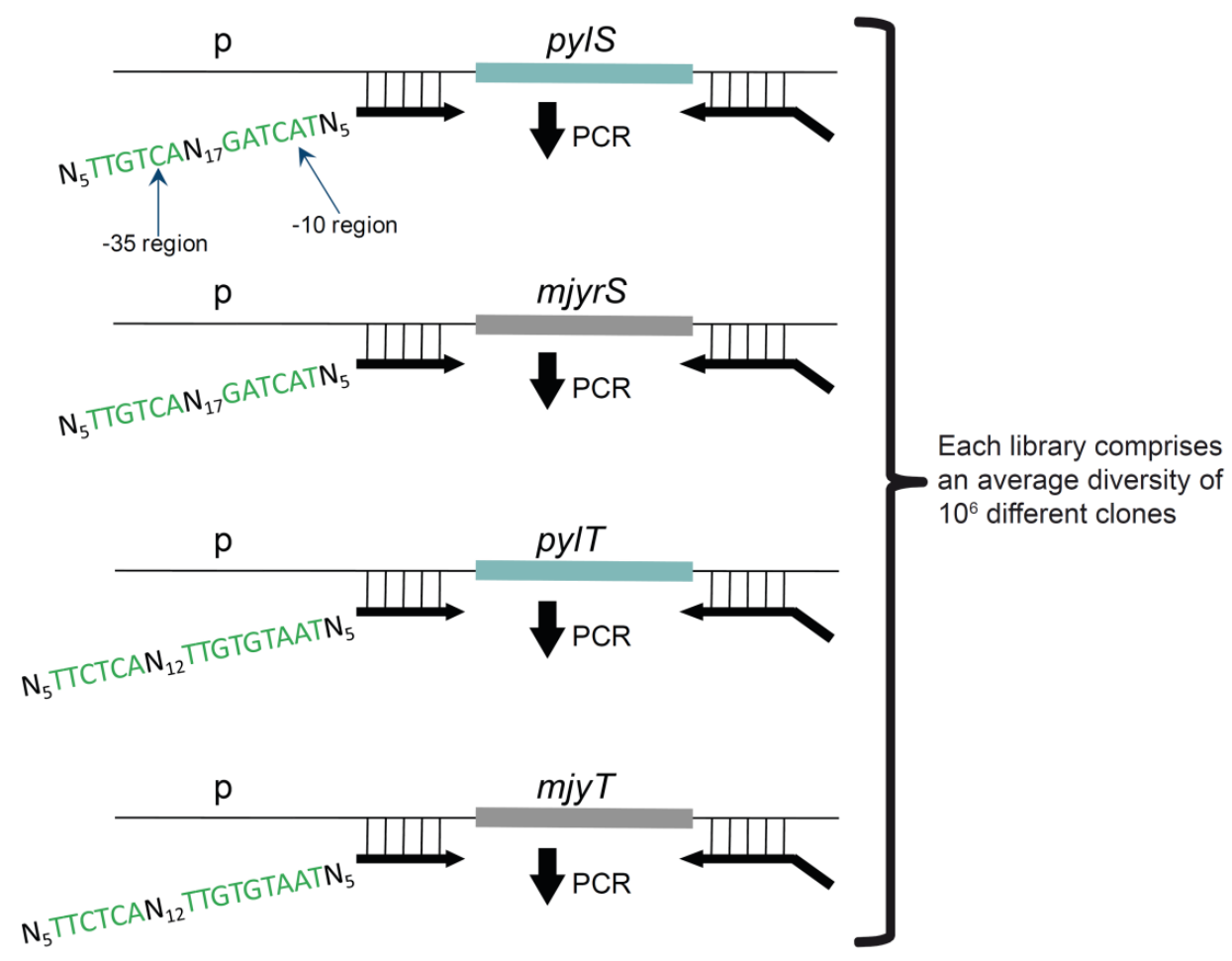

Figure 4.17: Schematic overview of the synthetic promoter libraries.

The promoters $(p)$ of the genes for pyIS, mjyrS_AGGA, pyIT and mjyT_UCCU were modified randomly via inverse PCR (Ch. 3.2.3.11). The -10 and the -35 box, highlighted in green, remained thereby unaffected. $N_{x}$ represents the number of exchanged nucleotides, with $\mathrm{N}$ standing for the bases $\mathrm{A}, \mathrm{T}, \mathrm{G}$ and $\mathrm{C}$. 
The mutagenesis of the $g / n S$ promoters in front of the aaRS and Ipp promoters ahead of the tRNAs, was performed using inverse PCR (Ch. 3.2.3.11). As depicted in Figure 4.17, mutations were applied to the nucleotides that surround the predicted -10 and -35 box, with the boxes themselves remaining unaffected. In detail, five bases upstream of the -35 box and further five bases downstream the -10 region were exchanged. Additionally, the 17 nucleotides connecting the two boxes in the glnS promoter and the 12 nucleotides in the Ipp promoter were randomly mutated. Jensen and Hammer observed a range of 400-fold change in activity by randomizing another promoter's spacer sequence between these two conserved boxes without affecting the length ${ }^{[160]}$. We designed the primers for the PCR in such a way that each modified position could be replaced randomly by any of the four nucleic acid bases. This yielded a theoretical diversity of $1.8 * 10^{16}$ different $g / n S$ promoters and $1.8^{*} 10^{13} \mathrm{lpp}$ promoters. The actual diversity achieved in these experiments covered an average of $10^{6}$ distinct clones.

\subsubsection{PylS Library}

The glnS promoter of the pylS gene (pCLA86; this plasmid is described in Ch. 4.4.2) was randomized as described above. We determined a diversity of $5 * 10^{6}$ different clones for this library. For the separation of inactive clones from active ones the amplified DNA was combined with a reporter plasmid (pCLA4) to select on chloramphenicol (Ch. 3.2.4.1). A dilution of cells was plated that consisted of $5^{*} 10^{0}, 5^{*} 10^{1}, 5 * 10^{2}$ cells on agar plates with $0 \mu \mathrm{g} / \mathrm{mL} \mathrm{Cm}$ and $5^{*} 10^{3}, 5^{*} 10^{5}, 5^{*} 10^{7}$ cells on plates with 25,50 and $100 \mu \mathrm{g} / \mathrm{mL} \mathrm{Cm}$ in the absence and presence of Bock. This was done for a clear discrimination of the effects from $\mathrm{Cm}$ and to obtain single clones for further investigations, e.g., broader range $\mathrm{Cm}$ assays. No growth could be observed on agar plates without Bock.

Cells were collected for the agar plates with Bock and the $5^{*} 10^{7}$ cell dilution in order to regain library plasmids from active clones and simultaneously remove reporter plasmids (Ch. 3.2.3.5). We chose the high cell count plates to assure the covering of the library diversity. Plasmid integrity was verified by restriction endonuclease digest (not shown).

As mentioned before, agar plates with low cell counts resulted in single colonies. In order to screen the diversity of the library 90 different clones from plates with 25,50 and $100 \mu \mathrm{g} / \mathrm{mL}$ $\mathrm{Cm}$ were transferred onto fresh agar plates with a broader range of $\mathrm{Cm}$, from 0 to $500 \mu \mathrm{g} / \mathrm{mL}$, allowing a more precise differentiation of these clones (Figure 4.18). 
Referring to Figure 4.18, a tendency of higher resistance to chloramphenicol from left to right could be observed. More cells on the right side of the agar plate survived the $\mathrm{Cm}$ concentration of $400 \mu \mathrm{g} / \mathrm{mL}$ in the presence of BocK, but also a Cm concentration of 75 to $100 \mu \mathrm{g} / \mathrm{mL}$ without Bock. This increase in resistance correlated with the amount of chloramphenicol that was existent in the original agar plates. Clones with the reference WT glnS promoter already showed a weaker growth at $250 \mu \mathrm{g} / \mathrm{mL}$.

Next, we addressed changes on the promoters that have positive effects on suppression efficiency. For this purpose, ten out of the 90 single library clones were analyzed by western and northern blots. Since all clones survived a $\mathrm{Cm}$ concentration of $250 \mu \mathrm{g} / \mathrm{mL}$ in the presence of Bock, we only selected clones that also survived $400 \mu \mathrm{g} / \mathrm{mL} \mathrm{Cm}$, or those that could not survive low concentrations of $\mathrm{Cm}$ without Bock. These were the clones 1D, $2 \mathrm{E}, 3 \mathrm{E}, 4 \mathrm{~F}, 6 \mathrm{~B}, 7 \mathrm{~A}, 8 \mathrm{G}, 10 \mathrm{D}, 11 \mathrm{D}$ and $12 \mathrm{H}$, according to the grid in Figure 4.18. The pBK PylS library plasmid variants were separated from the reporter plasmid (Ch. 3.2.3.5; not shown).

A) $\mathrm{Cm} 250+$ BocK

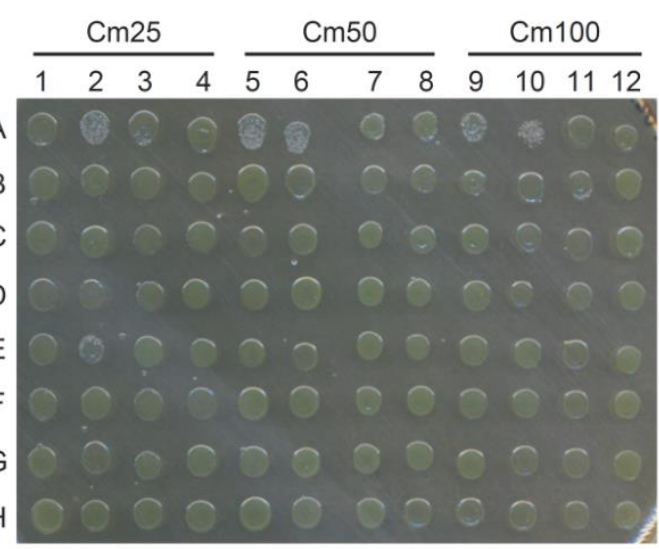

C) $\mathrm{Cm} 50$ - BocK

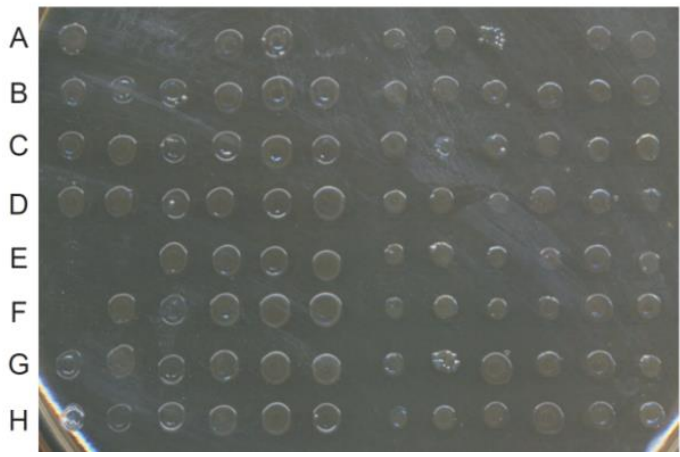

B) $\mathrm{Cm} 400+$ BocK

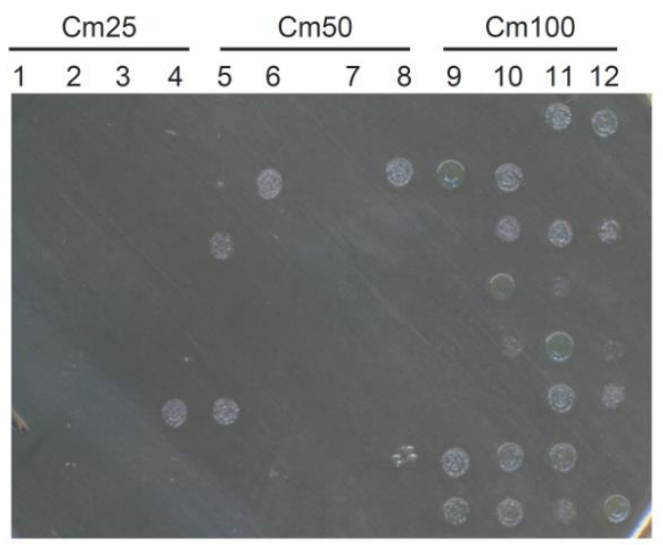

D) $\mathrm{Cm} 75$ - BocK

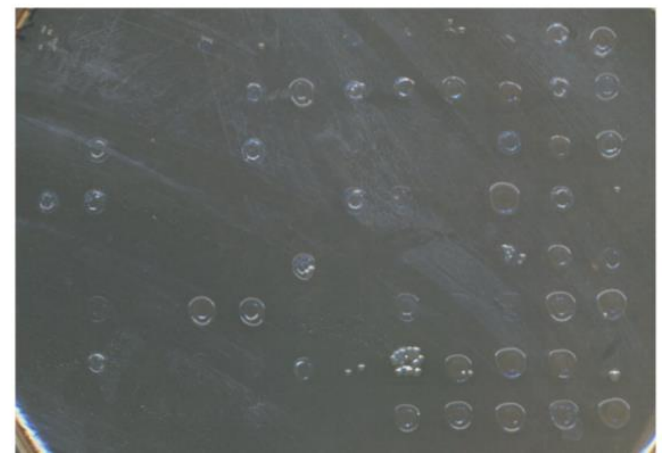

Figure 4.18: $\mathrm{Cm}$-Assay with single colonies from PylS library.

Cells were plated on agar plates containing Kan, Tet, increasing $\mathrm{Cm}$ and $1 \mathrm{mM}$ BocK ( $\mathbf{A}$ and $\mathbf{B}$ ) or no BocK (C and D). A selection of two plates each is shown only. The clones 1A, 5A and 9A contained PCLA9 as a reference and clones $2 \mathrm{~A}, 6 \mathrm{~A}$ and $10 \mathrm{~A}$ harbored $\mathrm{pCLA} 86$ as a reference. Clones in columns 1-4 originated from the library agar plate with $25 \mu \mathrm{g} / \mathrm{mL} \mathrm{Cm}$ and $1 \mathrm{mM}$ Bock, columns 5-8 from the plate with $50 \mu \mathrm{g} / \mathrm{mL} \mathrm{Cm}$ and $1 \mathrm{mM}$ Bock and columns 9-12 from the plate with $100 \mu \mathrm{g} / \mathrm{mL} \mathrm{Cm}$ and $1 \mathrm{mM}$ BocK. 
The purified library plasmids ( $\mathrm{PCLA22}$ to $\mathrm{pCLA31)}$ were sequenced to assure that all selected clones differed in sequence, but exclusively in the promoter region. Changes in the expression rate of PylS should then be due to mutations in the. The sequencing showed that beside a few silent mutations only those parts of the promoters were mutated which were intended to be. A sequence alignment of all ten library clones revealed that none of them had the same sequence (Figure 8.1).

Lastly, we transformed E. coli BL21 cells with the isolated library plasmids in combination with a vector that harbored the genes for the cognate tRNA PyIT and a $\mathrm{His}_{6}$-tagged histone H3 with an amber stop codon at position R52 (pCLA32). For suppression of the stop codon during the $\mathrm{H} 3$ expression the medium was supplemented with Bock. In the end, two samples of each culture were taken with one being used for comparative western blot analysis of expressed PyIS and histone H3. The other was used for northern blot to investigate if the library also had an effect on this level of the translational apparatus (Figure 4.19).

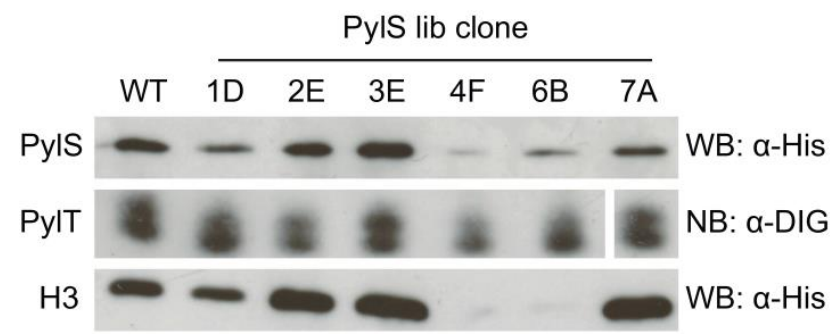

Figure 4.19: Comparative analysis of PyIS lib clones via western and northern blot.

For the investigation of the suppression efficiency of the library clones a histone H3 R52TAG (pCLA32) expression was performed. $1 \mathrm{mM}$ Bock was used as UAA and $\mathrm{H} 3$ expression was induced with $0.5 \mathrm{mM}$ IPTG at $\mathrm{OD}_{600}=0.8$. A selection of six different library clones (pCLA22 to pCLA27) was compared to WT PyIS, which comprised the unchanged $g / n S$ promoter (pCLA9). All samples were normalized by $\mathrm{OD}_{600}$. For the western blot whole cell extracts were separated with SDS PAGE (Ch. 3.2.2.3) and blotted onto a nitrocellulose membrane (Ch. 3.2.2.4). Anti-His-antibody was used as primary antibody. For the detection of the cognate tRNA PyIT total RNA was extracted from a second aliquot (Ch. 3.2.3.14). Approximately $0.090 \mu \mathrm{g}$ RNA were loaded for each sample. The DIG labeled probe C26 was utilized for hybridization and chemiluminescence signals recorded as described in $\mathrm{Ch}$. 3.2.3.15. The upper band in the second row represents the aminoacylated form of PylT, whereas the lower band depicts the uncharged form.

We found that the PyIS promoter library exhibited a variety of different promoter strengths. There were clones, for instance $3 \mathrm{E}$, that had an increased expression efficiency of the synthetase PyIS compared to the WT. On the other hand, we also observed clones, such as $4 \mathrm{~F}$, which displayed a strong decrease. Furthermore, the more PyIS was expressed in the cells, the more PylT in the aminoacylated, slower migrating form, could be observed. Finally, similar to the correlation of synthetase and cognate tRNA, we monitored a clear dependence on the amount of PyIS produced and the suppression efficiency, visualized by 
the amount of histone H3 R52TAG expressed. Hence, it was possible to influence the suppression efficiency of the amber stop codon with the aid of the PylS promoter library with an increase in PyIS resulting in higher expression levels of $\mathrm{H} 3$.

\subsubsection{MjYRS_AGGA Library}

The glns promoter of the synthetase MjYRS (frameshift codon version = AGGA) was mutated next ( $p C L A 72$; this plasmid is described in Ch. 4.4.2.). Unfortunately, the standard touchdown PCR protocol resulted in two PCR products, with the minority having the expected size of approximately $3000 \mathrm{bp}$ but the majority having a size of roughly $1200 \mathrm{bp}$. This effect was suspected to be due to unspecific primer annealing, despite the touchdown, and needed to be optimized. Therefore, we eliminated the touchdown portion of the PCR, i.e., the first ten cycles, and applied a gradient of increasing annealing temperatures. The gradient covered 54.5 to $66.5^{\circ} \mathrm{C}$, in twelve steps. This modified inverse gradient PCR was set up twice, with one set being supplemented with 5\% DMSO, which was thought to reduce the formation of secondary structures of template and primer DNA ${ }^{[161,162]}$.

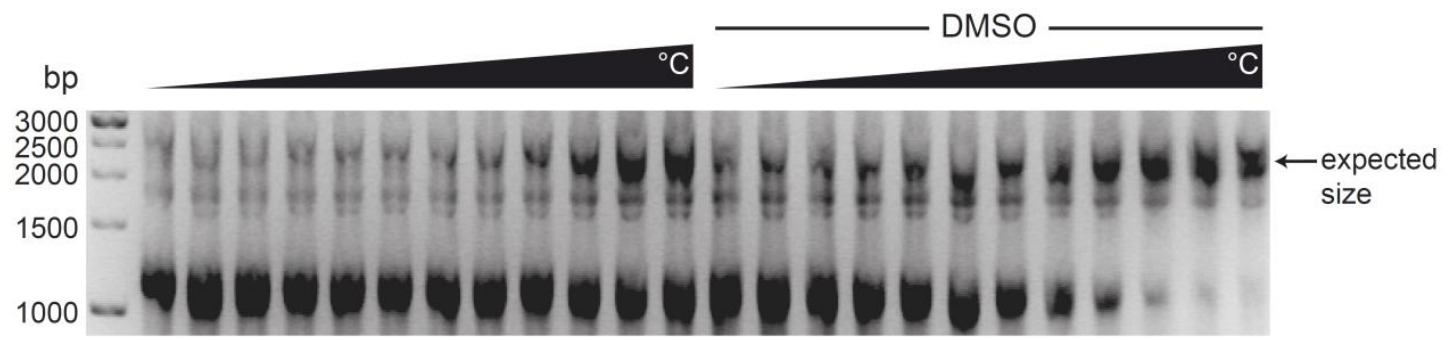

Figure 4.20: Inverse gradient PCR for the MjYRS promoter library.

Two sets were tested with 12 different temperatures each, ranging from 54.5 to $66.5^{\circ} \mathrm{C}$ (left to right; indicated by the black triangles). One set was supplemented with $5 \%$ DMSO. The expected size of the PCR product is approximately $3000 \mathrm{bp}$.

An increase of the annealing temperature caused a gain of the desired PCR product, as depicted in Figure 4.20. The addition of DMSO in combination with the high annealing temperature of $66.5^{\circ} \mathrm{C}$ finally led to a shift from undesired to the desired PCR product of expected size.

Cells transformed with the product of the PCR using these optimized parameters yielded $1.5^{*} 10^{6}$ different clones. Inactive clones were removed as described above for the PylS library using the $\mathrm{Cm}$ reporter ( $\mathrm{PCLA6}$ ). Since MjYRS, in the version described here, decodes for tyrosine, no control plates without the "UAA" could be made. However, a decreasing number of colonies formed on increasing $\mathrm{Cm}$ concentrations. The library DNA was again 
isolated from plates with $5 * 10^{7}$ cells and the isolation verified by restriction endonuclease digest (not shown).

The screening of the library diversity was the next step. To this end, we picked 30 different clones each from those single colony plates with 25,50 and $100 \mu \mathrm{g} / \mathrm{mL} \mathrm{of} \mathrm{Cm}$ and transferred them onto fresh agar plates with a broader range of $\mathrm{Cm}$, from 0 to $500 \mu \mathrm{g} / \mathrm{mL}$, (Figure 4.21).

Compared to the PylS library clones no tendency of higher resistance to chloramphenicol from left to right could be noticed. In contrast to PyIS, where the reference clones already showed weaker growth at $250 \mu \mathrm{g} / \mathrm{mL} \mathrm{Cm}$, the MjYRS WT promoter clones died more abruptly. None of the selected clones survived the highest concentration of $500 \mu \mathrm{g} / \mathrm{mL}$.

A) $\mathrm{Cm} 250$

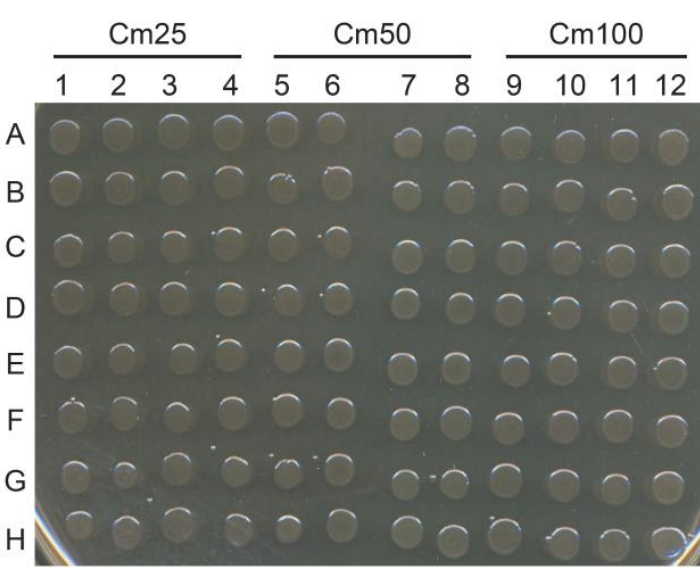

B) $\mathrm{Cm} 400$

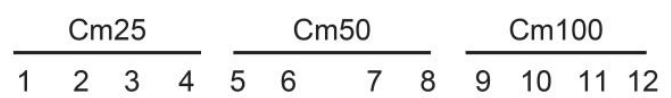

Figure 4.21: Cm-Assay with single colonies from MjYRS_AGGA library.

Cells were plated on agar plates containing Amp, Tet and increasing $\mathrm{Cm}$. A selection of two plates is shown only. The clones $1 \mathrm{~A}, 5 \mathrm{~A}$ and $9 \mathrm{~A}$ contained pCLA3 as a reference and clones $2 \mathrm{~A}, 6 \mathrm{~A}$ and $10 \mathrm{~A}$ harbored pCLA72 as a reference. Clones in columns 1-4 originated from the library agar plate with $25 \mu \mathrm{g} / \mathrm{mL} \mathrm{Cm}$, columns 5-8 from the plate with $50 \mu \mathrm{g} / \mathrm{mL} \mathrm{Cm}$ and columns 9-12 from the plate with $100 \mu \mathrm{g} / \mathrm{mL} \mathrm{Cm}$.

To investigate if the mutagenesis of the gInS promoter of the mjyrS gene had similar effects on the suppression efficiency as that of the mutant pylS promoter, ten clones out of the 90 were chosen. Since all the clones survived a $\mathrm{Cm}$ concentration of $250 \mu \mathrm{g} / \mathrm{mL}$ again, we considered how they behaved on the $\mathrm{Cm} 400$ plate. The DNA of the colonies 1D, 2F, 3B, 4A, $6 \mathrm{D}, 7 \mathrm{E}, 8 \mathrm{G}, 9 \mathrm{~F}, 10 \mathrm{~B}$ and $11 \mathrm{E}$, according to the grid in Figure 4.21, was isolated, because these clones represented a range of growth behavior. Thus, they were supposed to be able to repeat the distinct promoter strengths achieved with the PylS library. Therefore, the reporter plasmid was removed as previously described.

Sequencing of the purified library plasmids ( $p C L A 33$ to pCLA42) confirmed the intended mutagenesis of the nucleotides surrounding the predicted -10 and -35 box, with the boxes 
themselves remaining unaffected. A sequence alignment of all ten library clones revealed, that none of them had the same sequence (Figure 8.2).

For the classification of the library promoter strengths myoglobin with a frameshift codon at position S4 (pCLA43) was expressed. For this reason E. coli DH10B cells were transformed with the library plasmids pCLA33 to pCLA42 together with pCLA43, a vector that contained

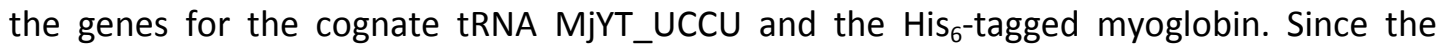
synthetase decodes for tyrosine no extra amino acid was added to the medium. Figure 4.22 shows the comparison of the ability to suppress the AGGA codon in case of myoglobin.

Regarding the blot in Figure 4.22 the expression levels of myoglobin fluctuated only in a small range, hardly distinguishable by eye. The quantification using ImageJ revealed a minimal signal intensity of $65 \%$ and a maximal intensity of $115 \%$ compared to wild type. In contrast to the PyIS library (Ch. 4.2.1), the MjYRS_AGGA promoter library seemed to have only a small influence on the suppression efficiency. Correlations between expressed synthetase and the protein of interest were not possible since the aaRS was not detectable on the blots.

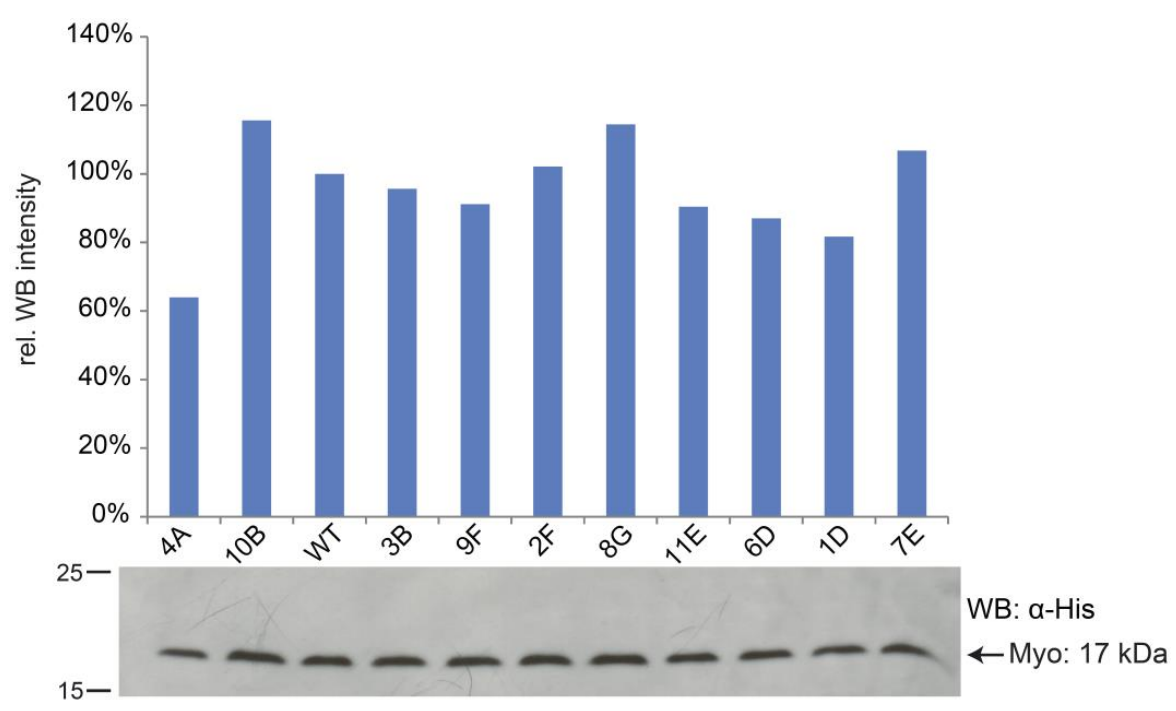

Figure 4.22: Suppression efficiency of MjYRS_AGGA lib clones.

The efficiency was determined by the expression of Myoglobin S4AGGA ( $p C L A 43$ ). Expression was induced with $0.2 \%$ arabinose $(\mathrm{w} / \mathrm{v})$ at $\mathrm{OD}_{600}=0.8$ and analyzed by western blot (lower panel) and the relative intensities measured by ImageJ (blue bars; upper panel; WT was set to $100 \%$ ). The ten library clones (pCLA33 to pCLA42)

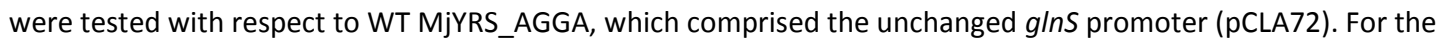
western blot all samples were normalized by $\mathrm{OD}_{600}$, whole cell extracts were separated with SDS PAGE (Ch. 3.2.2.3) and blotted onto a nitrocellulose membrane (Ch. 3.2.2.4). Anti-His-antibody was used as primary antibody. 


\subsubsection{PylT and MjYT_UCCU Library}

The last two promoters that had to be changed were the Ipp promoters of the $p y / T$ and mjYT_UCCU genes. The modification of the pylT promoter (pCLA79; this plasmid is described in Ch. 4.4.2) resulted in a library that held $5 * 10^{5}$ different clones. The mutagenesis on the M. jannaschii tRNA promoter (pCLA82; this plasmid is described in Ch. 4.4.2) yielded a diversity of $1.2 * 10^{6}$ distinct clones.

For the removal of inactive clones via $\mathrm{Cm}$ reporter assay the tRNA libraries had to be combined with their cognate aaRS. This is because they were cloned and mutated on the reporter plasmids themselves. The PyIT library was used for transformation together with the PyIS (pCLA1) and the MjYT_UCCU library together with the MjYRS_AGGA (pCLA3). Cells were plated as before (Ch. 4.2.1) with no extra UAA added to the agar plates for the M. jannaschii system and Bock added for the M. barkeri pair. The quantity of colonies generally decreased while increasing the $\mathrm{Cm}$ concentration. The DNA of both libraries was recovered and checked for correct sequence identity. In the case of PyIT a strong contamination with the parental reporter plasmid ( $p C L A 4)$ was found, which ran higher on an agarose gel (bands at 5625 and $4701 \mathrm{bp}$ ) than the mutagenized version (bands at 3928 and $1163 \mathrm{bp}$ ) used for making the library (data not shown). Before repeating the whole procedure, the usage of the restriction enzyme Ndel, which only cuts the parental plasmid, helped to clean the PyIT library DNA (that still included the inactive clones). Thus, both isolated libraries showed the correct sizes as confirmed by restriction enzyme digest (not shown).

We selected a total number of 96 different clones of each library from the single colony plates with 25,50 and $100 \mu \mathrm{g} / \mathrm{mL}$ of $\mathrm{Cm}$ to transfer them on fresh agar plates for the broad range $\mathrm{Cm}$ reporter assay (0 to $500 \mu \mathrm{g} / \mathrm{mL}$ ). The results for the PylT library are shown in Figure 4.23 and for the MjYT_UCCU library in Figure 4.24.

The control plates without BocK in Figure 4.23 showed that most of the PylT library clones were not able to survive concentrations of $\mathrm{Cm}$ higher than $75 \mu \mathrm{g} / \mathrm{mL}$ and all of them died at $150 \mu \mathrm{g} / \mathrm{mL}$. In the presence of BocK all clones could resist $250 \mu \mathrm{g} / \mathrm{mL}$, but only a few colonies in column I withstood $400 \mu \mathrm{g} / \mathrm{mL}$. These clones originated from the $\mathrm{Cm} 100$ single colony plate, a trait already observed for the PyIS library, but less distinctive.

Comparable to the MjYRS library, the MjYT_UCCU library clones displayed a wide range of growth efficiencies over the whole plate, independently from the original $\mathrm{Cm}$ concentration (Figure 4.24). Most of the clones resisted a $\mathrm{Cm}$ concentration of $400 \mu \mathrm{g} / \mathrm{mL}$, but none of 
them survived $500 \mu \mathrm{g} / \mathrm{mL}$. A few colonies became less dense at lower concentrations, e.g., the clones B3 and B6 already at $150 \mu \mathrm{g} / \mathrm{mL}$ (not shown) and the clones $\mathrm{H} 6$ and $\mathrm{J} 2$ at $250 \mu \mathrm{g} / \mathrm{mL}$.

A) $\mathrm{Cm} 250+$ BocK

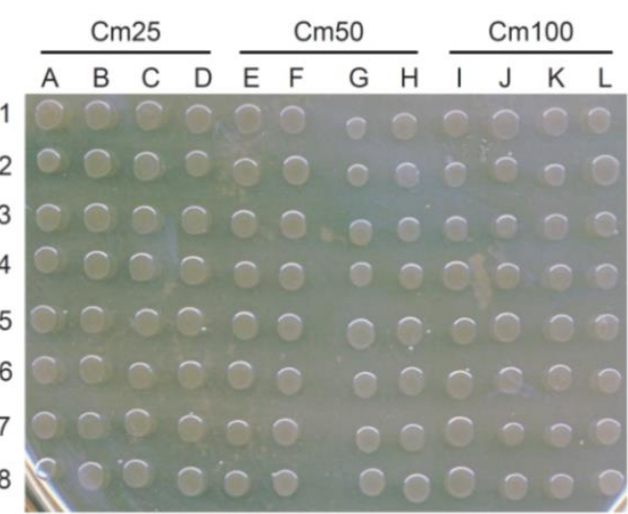

C) $\mathrm{Cm} 75$ - BocK

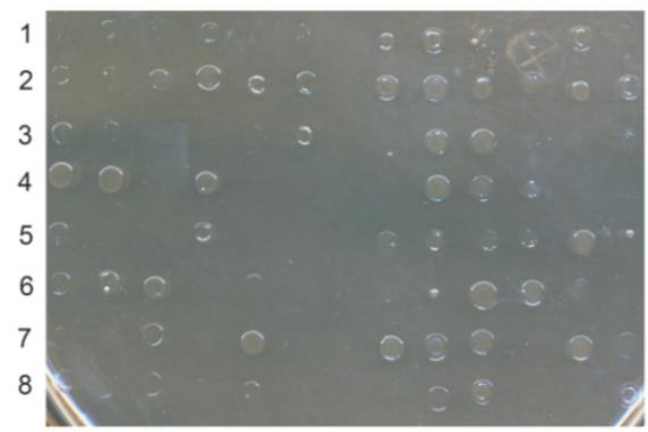

B) $\mathrm{Cm} 400+$ BocK

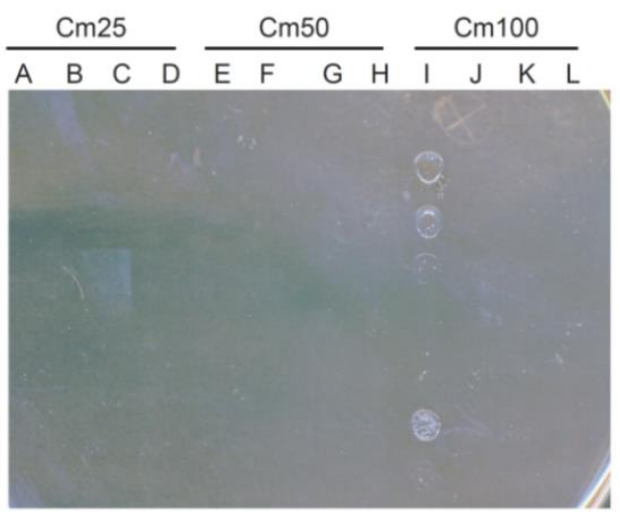

D) $\mathrm{Cm} 150$ - BocK

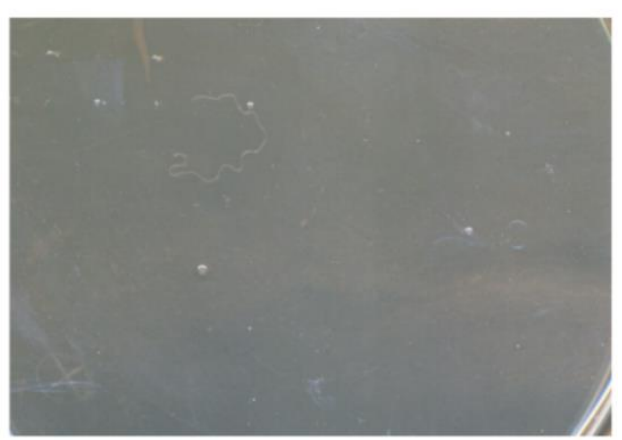

Figure 4.23: Cm-Assay with single colonies from PylT library.

Cells were plated on agar plates containing Kan, Tet, increasing $\mathrm{Cm}$ and $1 \mathrm{mM}$ BocK (A and $\mathbf{B}$ ) or no BocK (C and D). A selection of two plates each is shown only. Clones in columns A-D originated from the library agar plate with $25 \mu \mathrm{g} / \mathrm{mL} \mathrm{Cm}$ and $1 \mathrm{mM}$ Bock, columns E-H from the plate with $50 \mu \mathrm{g} / \mathrm{mL} \mathrm{Cm}$ and $1 \mathrm{mM}$ Bock and columns $\mathrm{I}-\mathrm{L}$ from the plate with $100 \mu \mathrm{g} / \mathrm{mL} \mathrm{Cm}$ and $1 \mathrm{mM}$ BocK.

A) $\mathrm{Cm} 250$

$\frac{C m 25}{A \quad B \quad C ~ D ~} \frac{C m 50}{\text { E F G H I J K L }}$

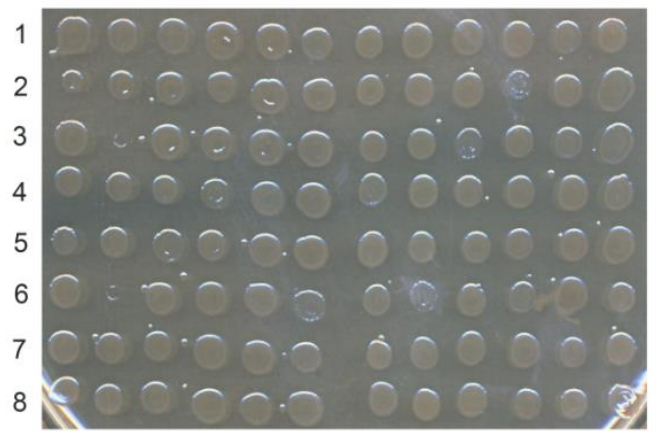

B) $\mathrm{Cm} 400$

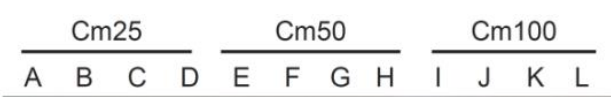

Figure 4.24: $\mathrm{Cm}$-Assay with single colonies from MjYT_UCCU library.

Cells were plated on agar plates containing Amp, Tet and increasing $\mathrm{Cm}$. A selection of two plates is shown only. Clones in columns A-D originated from the library agar plate with $25 \mu \mathrm{g} / \mathrm{mL} \mathrm{Cm}$, columns $\mathrm{E}-\mathrm{H}$ from the plate with $50 \mu \mathrm{g} / \mathrm{mL} \mathrm{Cm}$ and columns I-L from the plate with $100 \mu \mathrm{g} / \mathrm{mL} \mathrm{Cm}$. 
As before, ten out of the 96 clones of each tRNA library were selected to investigate effects on the suppression efficiency, due to changes in the Ipp promoter, with western and northern blots. The clones A4, C2, E7, F5, H4, 13, 16, J7, K2 and L5, according to the grid in Figure 4.23, were chosen for the PyIT library and their DNA separated from the synthetase plasmid (Ch. 3.2.3.5). Full separation was assured by the use of the restriction enzymes Ndel and Stul, which only cut the pBK synthetase plasmids (pCLA1 and pCLA3) but not the modified tRNA containing reporter plasmids (pCLA79 and pCLA82). The same was done for MjYT library clones B3, B8, D4, E1, G4, G7, 12, 18, K6 and L1, according to the grid in Figure 4.24. This procedure failed for PylT clone $\mathrm{H} 4$ and for MjYT clone G4, thus only nine clones per library were available (data not shown).

Finally, the sequencing of all purified tRNA library plasmids (pCLA44 to pCLA61) revealed that for both libraries, beside a few minor mutations, only those nucleotides surrounding the predicted -10 and -35 box were altered. The sequence alignment of each library illustrated and confirmed the diversity of the selected clones, with all of them being divergent (Figure 8.3 and Figure 8.4).

In conclusion, promoter libraries for each component of the two tRNA/aaRS pairs originating from the organisms $M$. barkeri and $M$. jannaschii have been prepared. The PylS library revealed a strong dependence of expressed synthetase on the expression of the protein of interest. When more synthetase was expressed it leads to an increased expression level for the desired protein. The MjYRS library did not display such a clear depenence. Individual studies of the tRNA library clones with western and northern blots have not been done, yet. Both libraries were used in combination with the synthetase libraries, as described in a later chapter (Ch. 4.4.4). 


\subsection{Defined (Standard) Promoters}

In chapter 4.2 we found that alterations of the PyIS synthetase promoter also effected the suppression efficiency of the amber codon inside a gene of a protein of interest and thereby the level of expression itself. The promoter of library clone $3 \mathrm{E}$ was among others the most powerful one and increased the levels of expressed PylS and histone $\mathrm{H} 3$ at least twofold (Figure 4.19). Hence, it was known that the promoter was stronger than the wild type $g$ InS variant, which was classified as a promoter of moderate strength ${ }^{[163]}$.

Two questions arose then: Is it possible to enhance the performance of the system once more by the usage of promoters characterized as strong, versus weak, and how can the library promoter be categorized in comparison to defined standard promoters like the E. coli lac promoter or the lambda $(\lambda)$ phage promoter? To this end a collection of different promoters was made whose strengths were already evaluated in literature. This set of promoters included endogenous E. coli promoters and their derivatives, coliphage promoters, as well as synthetic promoter variants. Their relative strength was mainly specified by so called $\mathrm{P}_{\text {bla-units }}{ }^{[164]}$. These units represent the ratio of RNA transcribed from a certain gene under the control of a promoter of interest with the transcripts of the $\beta$ lactamase gene $(b / a)$ under its endogenous promoter $\left(\mathrm{P}_{\mathrm{bla}}\right)$. For instance, the promoter $\mathrm{P}_{\mathrm{A} 1}$

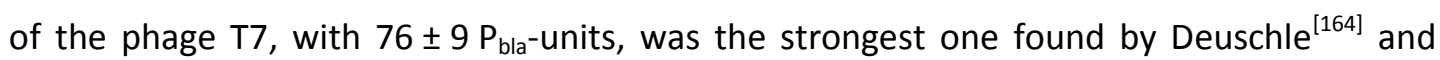

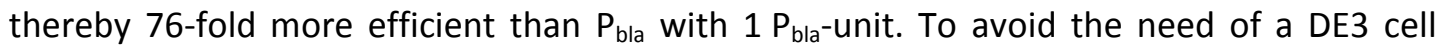
background, what means to be independent of the T7 RNA polymerase, no promoters of phage T7 were chosen. A list of the selected promoters together with their $\mathrm{P}_{\mathrm{bla}}$-units, if available, is shown in Table 4.1.

The sequences of the compiled promoters were cloned in front of the py/S gene (pCLA85; this plasmid is described in Ch. 4.4.2) while exchanging the glnS promoter at the same time.

We tested the capacity of these defined standard promoters concerning the suppression efficiency in the same way as we did for the PylS promoter library (Ch. 4.2.1) by expressing a His $_{6}$-tagged histone $\mathrm{H} 3$ with an amber stop codon at position R52. Thus, E. coli BL21 cells were transformed with the relevant pBK plasmids, including WT gInS (pCLA9) and library 3E promoter (pCLA24) as references, again in combination with the PyIT and H3 R52TAG genes containing pCDF plasmid (pCLA32). The expression was performed with BocK and the addition of IPTG started the synthesis of $\mathrm{H} 3$ as well as of PylS if provided with a $\mathrm{P}_{\text {lac }}$ derived promoter. All other promoters are constitutive and do not need an inducer. The comparative western blot is depicted in Figure 4.25. 
Table 4.1: Relative in vivo strength of selected promoters.

The strength of the promoters $(P)$ from various origins is given in $P_{b l a}$-units. The sequences were obtained from the mentioned references. No $P_{\text {bla }}$-units were found for $P_{\text {lac1-6 } M+5}$ and $P_{c p 25}$. $P_{\text {lac1-6 } M t 5}$ was described to have a relative transcription efficiency of $112 \%$ and is consequently 56 -fold stronger than $\mathrm{P}_{\text {lac }}$ that exhibited only $2{ }^{[165]}$. The synthetic promoter Pcp25 was merely characterized as "quite strong" without showing the data ${ }^{[160]}$. The strength of $P_{\lambda}$ found by Deuschle ${ }^{[164]}$ was corrected upwards by Knaus ${ }^{[166]}$.

\begin{tabular}{|c|c|c|c|}
\hline Origin & Promoter (P) & $\begin{array}{l}\text { Relative strength } \\
{\left[\mathbf{P}_{\mathrm{bla}}-\text { units }\right]}\end{array}$ & Reference \\
\hline \multirow{2}{*}{ Phage T5 } & $\mathrm{P}_{\mathrm{H} 207}$ & $55 \pm 4$ & Deuschle ${ }^{[164]}$ \\
\hline & $\mathrm{P}_{\mathrm{D} / \mathrm{E} 20}$ & $56 \pm 8$ & Deuschle $^{[164]}$ \\
\hline \multirow{2}{*}{ Phage lambda $(\lambda)$} & $\mathrm{P}_{\lambda}$ & $37 \pm 7 / 53 \pm 8$ & Deuschle $^{[164]} /$ Knaus $^{[166]}$ \\
\hline & $P_{\lambda c o n / N 25 D S R}$ & $65 \pm 9$ & Knaus ${ }^{[166]}$ \\
\hline \multirow{3}{*}{ E. coli } & $\mathrm{P}_{\mathrm{lac}}$ & $5.7 \pm 0.5$ & Deuschle $^{[164]}$ \\
\hline & $\mathrm{P}_{\mathrm{tacl}}$ & $17 \pm 2$ & Deuschle $^{[164]}$ \\
\hline & $\mathrm{P}_{\text {lac1-6 } \mathrm{Mt5}}$ & "56-fold of $\mathrm{P}_{\mathrm{lac}}$ " & $\operatorname{Liu}^{[165]}$ \\
\hline \multirow{2}{*}{ Synthetic } & $\mathrm{P}_{\text {con }}$ & $4 \pm 0.2$ & Deuschle $^{[164]}$ \\
\hline & $P_{c p 25}$ & "quite strong" & Jensen $^{[160]}$ \\
\hline
\end{tabular}

Our initial observations suggested that the expression of the synthetase PylS worked for all the different promoters tested except $P_{c p 25}, P_{\lambda c o n / N 25 D S R}$ and $P_{H 207}$ ( Figure 4.25). For $P_{c p 25}$ no signal could be detected, but the $\mathrm{P}_{\lambda \mathrm{con} / \mathrm{N} 25 \mathrm{DSR}}$ promoter samples revealed a relatively intense band smaller than the expected size of $48 \mathrm{kDa}$ probably due to degradation. The $\mathrm{P}_{\mathrm{H} 207}$ lane showed a very weak signal of the same reduced size.

The various promoters were in control of a broad spectrum of produced PylS, ranging from small amounts, in the case of the WT promoter, up to a multiple fold increases of these

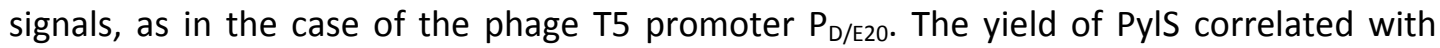
most of the relative promoter strengths given in Table 4.1. We noticed the lowest abundances for the gInS WT promoter, which was characterized as moderate ${ }^{[163]}$ before, and the library promoter $\mathrm{P}_{\text {lib3E }}$ followed by the consensus sequence based $\mathrm{P}_{\text {con, }}$, which had the fewest $\mathrm{P}_{\mathrm{bla}}$-units of all. $\mathrm{P}_{\mathrm{lac}}$ was the next strongest candidate referring to $\mathrm{P}_{\mathrm{bla}}$-units, what also coincided with the signal intensities on the blot. The modified lac promoter variant $P_{\text {lac1-6 Mt5 }}$ seemed to be more powerful than its precursor, but not to that extent as stated by $\mathrm{Liu}^{[165]}$. The phage promoters appeared to be the overall strongest ones, as expected by $\mathrm{Pbla}^{-}$ units. In previously performed experiments (not shown) $\mathrm{P}_{\lambda \text { con/N25DSR }}$ already turned out to be the most powerful promoter, according to the $65 \mathrm{P}_{\mathrm{bla}}$-units, assuming that the mass of degraded PylS protein had a high transcription rate as a cause. However, despite its strength this promoter was not successful because no full-length PyIS was produced in the 
end. Regarding the blot, the promoter hybrid $\mathrm{P}_{\text {tacl }}$ revealed higher PylS intensities as anticipated with respect to $P_{\text {bla }}$-units, arranging between $P_{\lambda}$ and $P_{D / E 20}$.

We then observed a strong correlation of produced PylS and expressed histone H3 R52TAG, similar to the PyIS library clones (Figure 4.19). This means, that with an increasing level of PylS the level of histone increased as well. Nevertheless, there seemed to be a certain limit

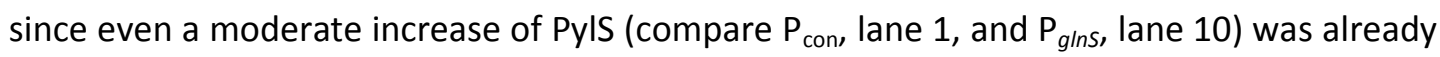
beneficial, while yields of histone H3 were not further improved, if more PylS was expressed. In contrast to the current result $\mathrm{P}_{\text {lac }}$ has been shown in previous trials (not shown) to mediate histone $\mathrm{H} 3$ amounts similar to $\mathrm{P}_{\text {con. }}$.

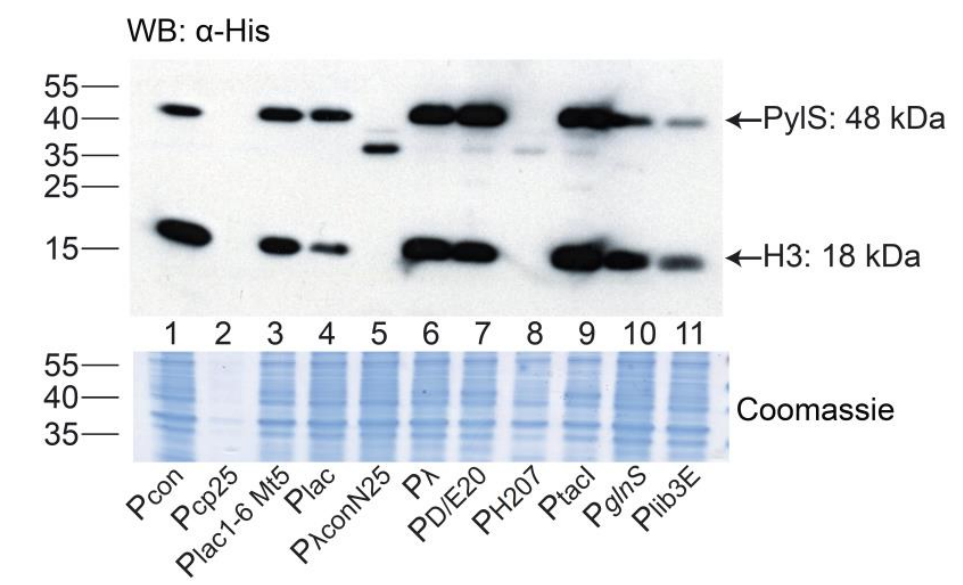

Figure 4.25: Comparative analysis of defined standard promoters with WT gInS and library clone 3E promoter. The suppression efficiency in histone H3 R52TAG (pCLA32) expression of systems under the control of defined standard promoters ( $p C L A 62$ to pCLA70) was compared to systems where the aaRS PyIS was expressed under the control of the WT gInS (pCLA9) or library clone 3E promoter (pCLA24). $1 \mathrm{mM}$ Bock was used as UAA and H3 expression was induced with $0.5 \mathrm{mM}$ IPTG at $\mathrm{OD}_{600}=0.8$ as well as PylS that was dependent on $\mathrm{P}_{\text {lac }}$ promoter and its derivatives. All samples were normalized by $\mathrm{OD}_{600}$. For the western blot whole cell extracts were separated with SDS PAGE (Ch. 3.2.2.3) and blotted onto a PVDF membrane (Ch. 3.2.2.4). Anti-His-antibody was used as primary antibody.

Referring to the two questions at the beginning of this chapter, the library promoter $3 \mathrm{E}$ and also the WT glnS promoter could be classified as weak, if compared to the power of the other standard promoters. Furthermore, it was possible to significantly improve the suppression efficiency of the given system by using strong promoters. However, we considered $P_{\text {con }}$ and $P_{\text {lac }}$ to use in later experiments (Ch. 4.4.6) because the former one provided an enhancement of the system comparable to the strongest promoters and the latter one offered the advantage of an inducible promoter, what could be essential if a product is (very) toxic to the host cell. 


\subsection{The Modular Genetic Tool}

\subsubsection{The Concept of The Modular Genetic Tool}

In chapters 4.2 and 4.3 we tried to optimize the suppression efficiency of amber stop codons or frameshift codons by the use of promoter libraries. A change of the accordant synthetase expression level was intended to have an effect on the expression rate of a protein of interest that contains the nonsense or quadruplet codon in its gene. Several promoters were found, especially for PylS, which improved the suppression efficiency.

Whereas the optimization trials mentioned above focused on the incorporation of a single UAA, the simultaneous insertion of two or more UAAs was the greater goal. For the production of proteins containing multiple UAAs there requires an equivalent number of tRNA/aaRS pairs. These pairs are typically harbored on a single plasmid, where one would need two plasmids to accommodate for the suppression of two unique amino acids. In conjunction with the plasmid for the protein of interest, a minimum of three different plasmids is needed to produce a protein with two UAAs. Three plasmids also imply the use of three distinct antibiotics leading to increased stress levels in host cells.

Moreover, the incorporation of two different UAAs decreases the expression efficiency once more. Neumann and colleagues showed an approach to overcome this deficit by the application of an evolved orthogonal ribosome that efficiently decodes a series of quadruplet codons as well as the amber codon ${ }^{[78]}$. The disadvantage of this method is the necessity for yet another plasmid that contains the orthogonal ribosomal RNA. In summary, although the expression efficiency is improved, the total count of plasmids and antibiotic stress is increased in host cells.

Hence, another possibility for the optimization of the genetic code expansion system is given by reducing the number of plasmids used. This should be achieved by combining both tRNA/aaRS pairs on a single vector based on pCDF Duet-1 as depicted in Figure 4.26-A. Furthermore, this plasmid should provide the opportunity to exchange each component for other tRNA/aaRS pairs, individually, to introduce new UAAs. Finally, even the replacement of each promoter and terminator sequence of all four components should be available, resulting in a highly modular genetic tool (Figure 4.26-B). The Schultz laboratory pursued a similar approach by combining an amber codon suppressing MjTyr pair and an ochre suppressing pyrrolysine pair on an evolved suppressor plasmid ${ }^{[72]}$. They were able to efficiently incorporate BocK and $p$-acetyl-L-phenylalanine (pAcF) into GFP albeit GFP levels did not exceed those if both suppression systems were split on two vectors. 
A)

B)
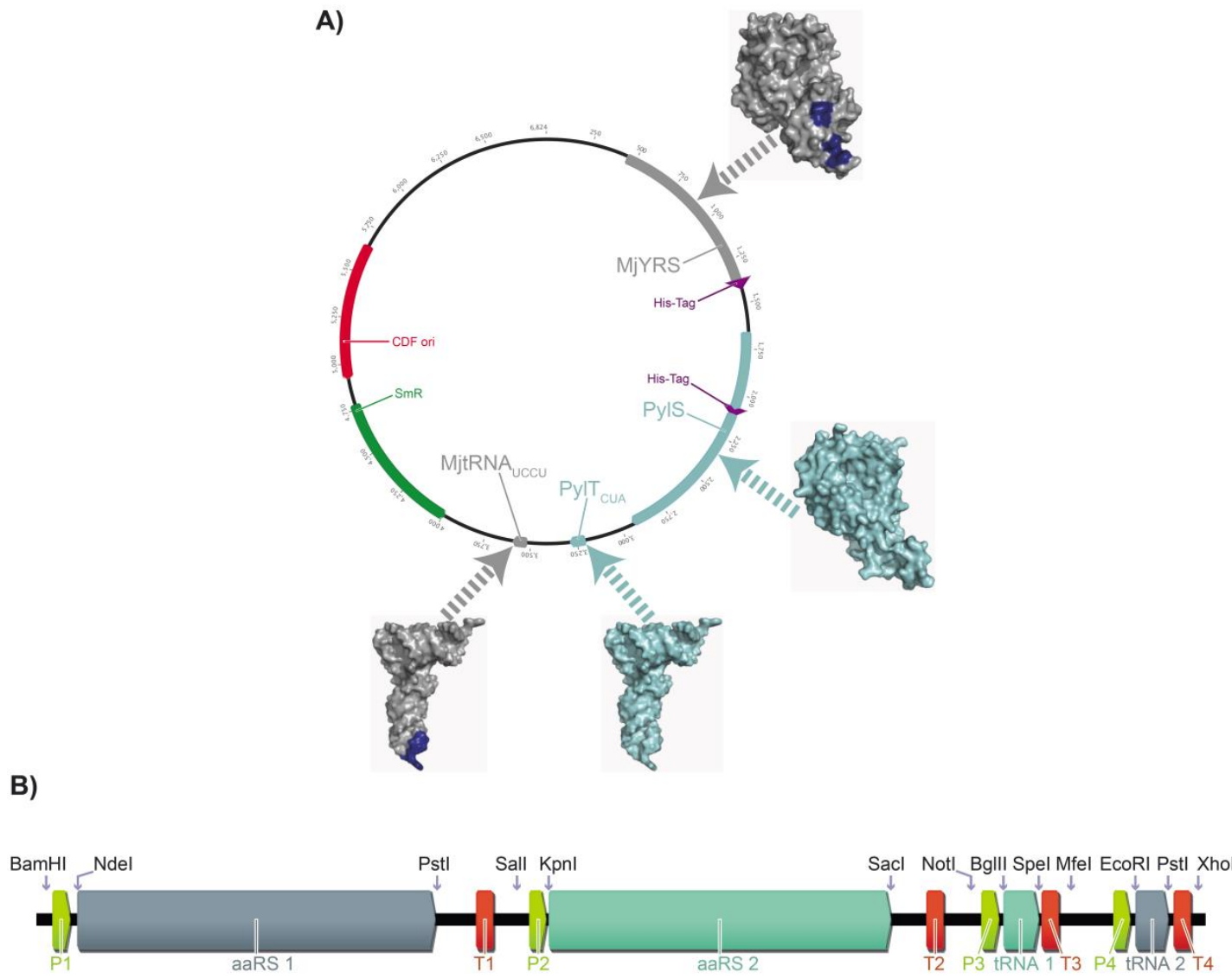

Figure 4.26: Schematic overview of the modular genetic tool.

A) The genes mjyrS_AGGA, pylS, pylT and mjyT_UCCU will be combined on a single vector based on pCDF Duet-1 (Novagen). This will reduce the number of plasmids required for the incorporation of two UAAs and the needed antibiotics at the same time by one. B) For the construction of the modular genetic tool that allows the replacement of every single component, including their promoters (P1-4; light green) and terminators (T1-4, orange), a series of unique restriction sites is mandatory.

\subsubsection{The Construction of The Modular Genetic Tool}

The construction of the modular genetic tool started with the incorporation and/or the exchange of the numerous unique restriction sites according to Figure 4.26-B. To this end, a series of twelve different QuikChanges (Ch. 3.2.3.10) was performed that mutated the basic

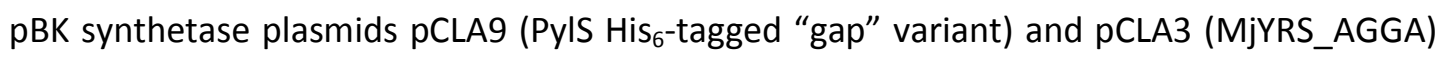
as well as the pREP plasmids for the tRNAs PyIT (pCLA4) and MjYT_UCCU (pCLA6). Table 4.2 shows a list of all applied QCs including plasmids that were used for mutagenesis. Every cloning step was checked for success via restriction digest using the appropriate enzymes (data not shown) and sequencing data revealed that all twelve QCs were successfully mutated. 
Table 4.2: Overview of all QuikChanges performed in order to construct the modular genetic tool. Twelve different QCs were made in total. The number of the QC is aligned with the used template, the purpose of the QC and the resulting plasmid. Except for pCLA77 and pCLA80 all produced plasmids were sequenced. Details of the construction (primers and sequencing primers) can be found in Table 8.3.

\begin{tabular}{|c|c|c|c|c|}
\hline & $\begin{array}{l}\text { QC } \\
\#\end{array}$ & Template & Purpose & Product \\
\hline \multirow{2}{*}{ 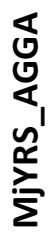 } & 1 & pCLA3 & $\begin{array}{l}\text { C-terminal } \mathrm{His}_{6} \text {-tag on } \\
\text { MjYRS_AGGA }\end{array}$ & pCLA71 \\
\hline & 2 & pCLA71 & $\begin{array}{l}\text { Sall restriction site behind MjYRS } \\
\text { terminator }\end{array}$ & pCLA72 \\
\hline \multirow{4}{*}{$\frac{n}{a}$} & 3 & pCLA9 & $\begin{array}{l}\text { Ndel restriction site in front of } \\
\text { PylS start codon changed to Kpnl }\end{array}$ & pCLA73 \\
\hline & 4 & pCLA73 & $\begin{array}{l}\text { Pstl restriction site behind PyIS } \\
\text { stop codon changed to Sacl }\end{array}$ & pCLA74 \\
\hline & 5 & pCLA74 & $\begin{array}{l}\text { Sall restriction site in front of } \\
\text { PylS promoter }\end{array}$ & pCLA75 \\
\hline & 6 & $\mathrm{pCLA75}$ & $\begin{array}{l}\text { Notl restriction site behind PyIS } \\
\text { terminator }\end{array}$ & $\mathrm{pCLA76}$ \\
\hline \multirow{3}{*}{ 上 } & 7 & pCLA4 & $\begin{array}{l}\text { deletion of genes for T7 RNA } \\
\text { Polymerase, AraC and GFP }\end{array}$ & pCLA77 \\
\hline & 8 & $\mathrm{pCLA} 77$ & $\begin{array}{l}\text { Notl restriction site in front of } \\
\text { PylT promoter }\end{array}$ & $\mathrm{pCLA78}$ \\
\hline & 9 & pCLA78 & $\begin{array}{l}\text { Mfel restriction site behind PylT } \\
\text { terminator }\end{array}$ & pCLA79 \\
\hline \multirow{3}{*}{ 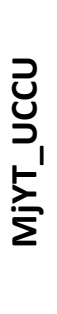 } & 10 & pCLA6 & $\begin{array}{l}\text { deletion of genes for T7 RNA } \\
\text { Polymerase, AraC and GFP }\end{array}$ & pCLA80 \\
\hline & 11 & pCLA80 & $\begin{array}{l}\text { Mfel restriction site in front of } \\
\text { MjYT_UCCU promoter }\end{array}$ & pCLA81 \\
\hline & 12 & pCLA81 & $\begin{array}{l}\text { Xhol restriction site behind } \\
\text { MjYT_UCCU terminator }\end{array}$ & pCLA82 \\
\hline
\end{tabular}

The extent of the mutagenesis in the preparation of the modular genetic tool holds a certain amount of risk because one or more mutations could influence the efficiency of the system negatively. Thus, chloramphenicol reporter assays were made to test the final plasmids pCLA72, pCLA76, pCLA79 and pCLA82 as well as the intermediate steps. Most of the plasmids revealed the same suppression efficiency as the basic plasmid (data not shown), except for PyIS (Figure 4.27). Independent of the plasmid used for transformation, the cells were not able to survive $\mathrm{Cm}$ concentrations higher than $25 \mu \mathrm{g} / \mathrm{mL}$. However, cells that harbored the unchanged basic plasmid ( $p C L A 9$ ) were still able to grow on agar plates with $400 \mu \mathrm{g} / \mathrm{mL}$ chloramphenicol. 


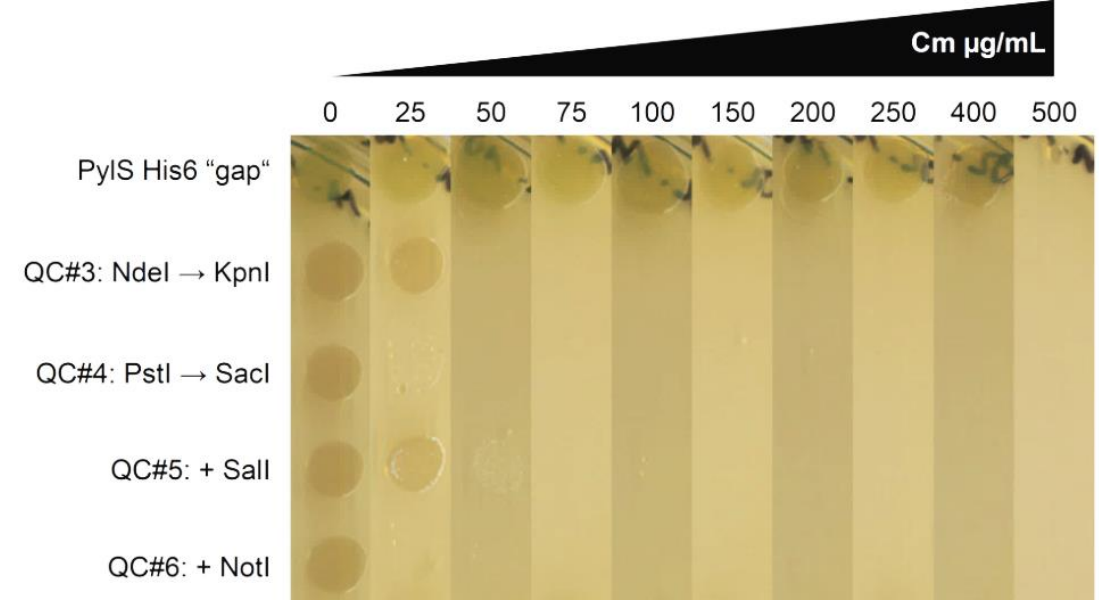

Figure 4.27: Cm-Assay with final pBK PyIS plasmid and intermediate steps from preparation for the modular genetic tool.

The catalytic activity of PyIS His 6 "gap" on the basic plasmid (pCLA9) was compared to PyIS on the mutated plasmids pCLA73 to pCLA76 from QC\#3-6 (Table 4.2). Therefore a chloramphenicol reporter assay (Ch. 3.2.4.1) was performed and cells were plated on agar plates containing Kan, Tet, increasing $\mathrm{Cm}$ and $1 \mathrm{mM}$ Bock. Only cells with the basic plasmid were able to survive $\mathrm{Cm}$ concentrations higher than $25 \mu \mathrm{g} / \mathrm{mL}$.

Since the activity already decreased after the first $Q C$ that exchanged the Ndel restriction with Kpnl and the resulting plasmid served as precursor for the following QCs, we needed to determine if the catalytic activity of PyIS itself was disturbed or if there was another cause for this effect. For this reason QC\#5 and \#6 (Table 4.2) were repeated using the basic plasmid (pCLA9) as template this time (QC\#5B: pCLA83 and \#6B: pCLA84), in order to incorporate the corresponding sites in a different order. Additionally, two further clones of QC\#3 were tested in a subsequent $\mathrm{Cm}$ reporter assay (Figure 4.28).

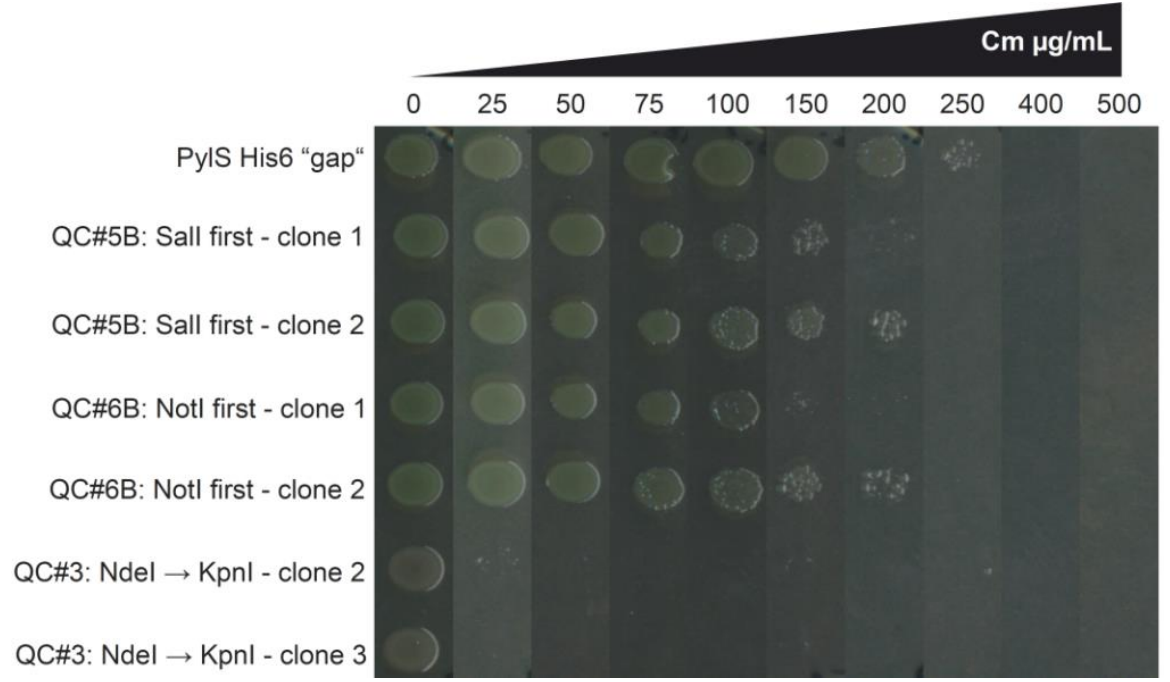

Figure 4.28: $\mathrm{Cm}$-Assay to detect the stage responsible for PylS activity loss.

The catalytic activity of PyIS $\mathrm{His}_{6}$ "gap" on the basic plasmid (pCLA9) was compared to PylS on the mutated plasmids pCLA73, pCLA83 and pCLA84 from QC\#3, \#5B and \#6B (two clones each). Therefore a chloramphenicol reporter assay (Ch. 3.2.4.1) was performed and cells were plated on agar plates containing Kan, Tet, increasing $\mathrm{Cm}$ and $1 \mathrm{mM}$ Bock. Only cells with the plasmid from QC\#3 were not able to survive $\mathrm{Cm}$ concentrations of $25 \mu \mathrm{g} / \mathrm{mL}$. 
It could be stated that the incorporation of the restriction sites Sall and Notl alone did not perturb the suppression efficiency of PyIS as depicted in Figure 4.28. In contrast, the two other clones of QC\#3 tested, showed the same phenotype as before. Hence, the replacement of the Ndel site upstream of the PylS start codon by Kpnl seemed to be the reason for the PyIS activity loss.

It was necessary to exchange, or at least to destroy, the Ndel site at the PylS gene for the construction of the modular genetic tool because the gene for MjYRS_AGGA also possesses an Ndel site. We then asked two questions. First, is it possible to restore the PylS activity on pCLA76 by mutating the Kpnl site back to Ndel? Second, is it possible to restore the activity and destroy the Ndel site simultaneously? A series of six different QCs was applied to pCLA76, one to restore the Ndel site and the other ones to restore the site while introducing point mutations ( $\mathrm{PCLA} 85$ to pCLA90). The Ndel-restore mutants were again screened via $\mathrm{Cm}$ reporter assay (Figure 4.29).

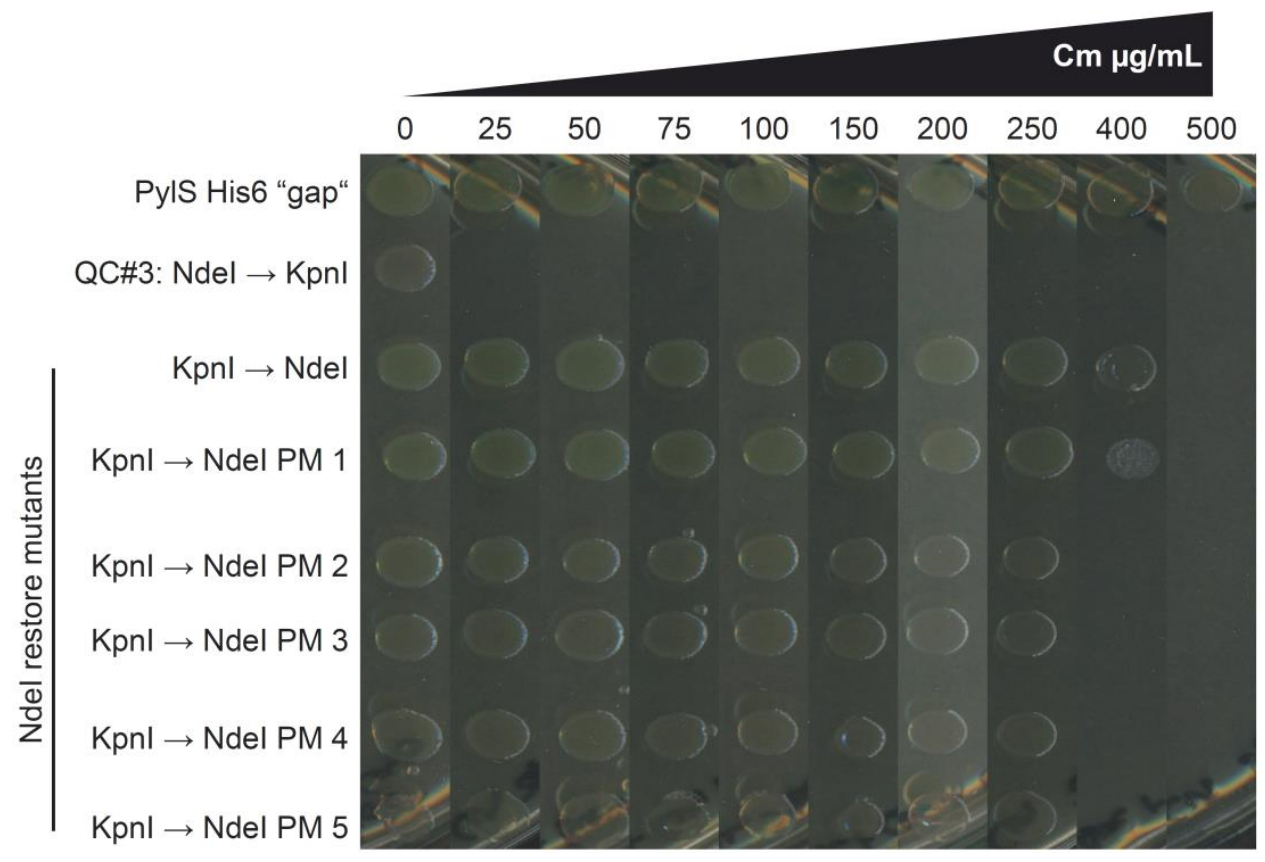

Figure 4.29: $\mathrm{Cm}$-Assay of the PyIS Ndel restore mutants.

The catalytic activity of PyIS His "gap" on the basic plasmid (pCLA9) was compared to PylS on the mutated plasmids pCLA73 (QC\#3), pCLA85 (Ndel restore) and pCLA86 to pCLA90 (Ndel restore Point Mutations (PM) 1-5). Therefore a chloramphenicol reporter assay (Ch. 3.2.4.1) was performed and cells were plated on agar plates containing Kan, Tet, increasing $\mathrm{Cm}$ and $1 \mathrm{mM}$ BocK. Only cells with the plasmid from QC\#3 were not able to survive $\mathrm{Cm}$ concentrations of $25 \mu \mathrm{g} / \mathrm{mL}$.

The activity of PylS could be almost restored by reinstalling the Ndel site in front of the PylS start codon. The PylS activity of the point mutation variants of Ndel was similar to the fully reconstituted Ndel site, as shown in Figure 4.29. Moreover, the point mutated Ndel sites were not digested by the restriction enzyme Ndel any more (data not shown). Due to the 
highest PyIS activity of all point mutation clones, pCLA86 (Ndel-restore_PM1) was chosen for further experiments.

Since all needed restriction sites were cloned into the basic $\mathrm{pBK}$ and $\mathrm{pREP}$ vectors, according to Figure 4.26-B and Table 4.2, we performed digests in a preparative scale (Ch. 3.2.3.2; Table 4.3) to cut out the genes for both tRNA/aaRS pairs and combined it on a pCDF Duet-1 backbone (Novagen; pCLA91).

Table 4.3: Overview of preparative digests performed in order to construct the modular genetic tool. Plasmids for inserts and backbones were digested with the same enzymes. The insert is equal to the component mentioned in the table. The plasmid pCLA86 had to be cut by three restriction enzymes to assure the full separation of backbone and insert on a subsequent agarose gel. Cutting with Sall and Notl only would yield bands with sizes of 1555 and $1707 \mathrm{bp}$, which are difficult to excise from gel precisely.

\begin{tabular}{lllll}
\multicolumn{2}{c}{ Plasmid } & Enzymes & Component & Product \\
Insert & Backbone & & & \\
\hline pCLA72 & pCLA91 & BamHI + Sall & MjYRS_AGGA & pCLA92 \\
\hline pCLA86 & pCLA92 & Sall + Notl (+ Xhol) & PylS & pCLA93 \\
\hline pCLA79 & pCLA93 & Notl + Mfel & PylT & pCLA94 \\
\hline pCLA82 & pCLA94 & Mfel + Xhol & MjYT_UCCU & pCLA95
\end{tabular}

All four components could be successfully transferred into the pCDF Duet-1 vector. Plasmid integrity was verified by restriction enzyme digests (Figure 4.30) and sequencing.

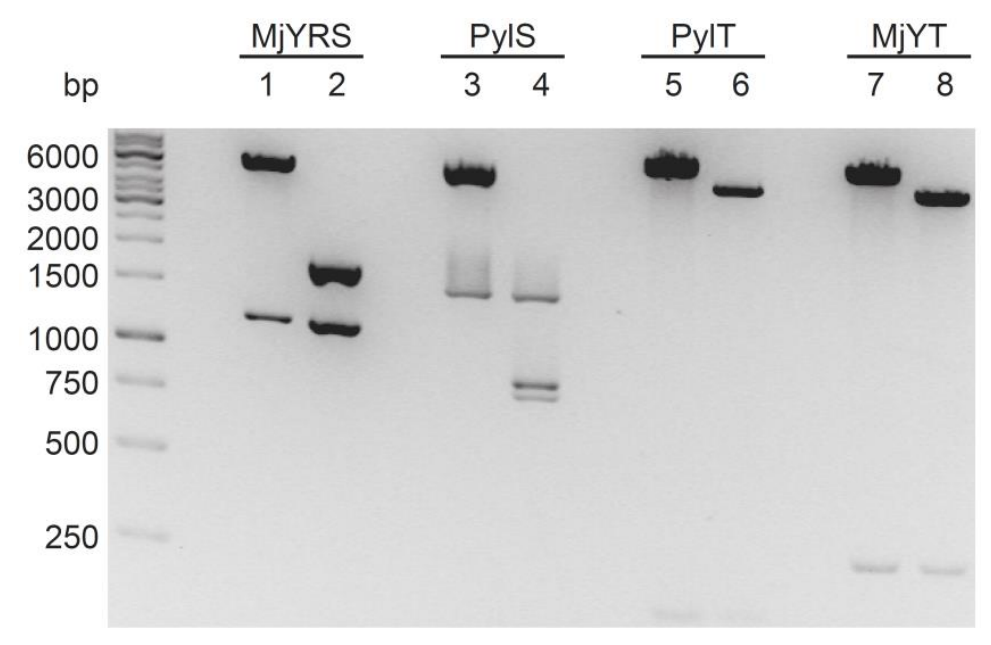

Figure 4.30: Restriction digests of the final modular genetic tool.

The final plasmid pCLA95 that contains all four components (digests 1, 3, 5 and 7) was compared to the insert plasmids (Table 4.3) pCLA72 (digest 2), pCLA86 (digest 4), pCLA79 (digest 6) and pCLA82 (digest 8). Enzymes and sizes: BamHI and Sall ( $1=5618$ and 1206; $2=1820$ and 1206); Sall and Notl $(3=5269$ and 1555; $4=1555,890$ and 817); Notl and Mfel ( $5=6612$ and 212; $6=4879$ and 212); Mfel and Xhol ( $7=6506$ and 318; $8=4776$ and 318). 


\subsubsection{Monitoring The Power of The Modular Genetic Tool}

In order to determine if the modular genetic tool had benefits compared to the classical system using four different plasmids, it had to be tested using a reporter system. Therefore, we established a second reporter in parallel to the Cm assay - the GFP reporter assay (Ch. 3.2.4.2). This assay exists in two variants, with variant 1 consisting of three plasmids, namely the modular genetic tool (pCLA95), a plasmid for the orthogonal ribosome riboQ1 ${ }^{[78]}$ (pCLA96) and finally one out of four different GFP reporter plasmids (pCLA105 to pCLA108). The second variant only differed by the separation of the four components of the genetic tool onto two plasmids, namely pCDF PyIST ${ }^{[78]}$ (pCLA97) and pDULE MjYRST (this version decodes for UCCU and is based on the CUA version ${ }^{[68,83]}$; pCLA98).

For the construction of the GFP reporter a plasmid made by Wang and others, called pO-gst-malE ${ }^{[67]}$ (pCLA99), was taken as a basis. This vector contains a mutated ShineDalgarno sequence that is only bound by orthogonal ribosomes (ORBS = orthogonal ribosomal binding site), in this case the ribo-Q1 provided on pCLA96. As a first step the gene for the fusion protein GST-MBP was removed, except for the first five triplet codons to assure that the binding of the orthogonal ribosome is not perturbed in any way. Simultaneously, a small multiple cloning site (MCS) was introduced, including the unique restriction sites for Ncol, BamHI, Notl and HindIII. The latter one was embedded in the final ochre stop codon of the open reading frame. The plasmid (pCLA100) was controlled by restriction digest and sequencing. Next, we amplified the actual reporter genes encoding for superfolder GFP ${ }^{[167]}$ from pBAD sfGFP vectors, made by Stokes et al. ${ }^{[168]}$, including four different genes for a wild type version, a gene with an amber codon at position N150, a frameshift codon at position D134 or both substitutions on one gene at the same time. All versions contained a C-terminal $\mathrm{His}_{6}$-tag and the former restriction site, $\mathrm{Xhol}$, at the end of the gene, was changed to Hindlll during the PCR, allowing the cloning into the ORBS reporter pCLA100 subsequent to restriction digest (pCLA105 to pCLA108).

Four sets of electro competent cells were prepared (Ch. 3.2.1.2), with all of them containing the plasmid for the orthogonal ribosome ribo-Q1 (pCLA96) and one out of the four different ORBS GFP reporter plasmids (pCLA105 to pCLA108). For the comparison of the three plasmid system, variant 1 , and the four plasmid system, variant 2 , these competent cells were transformed with appropriate plasmids and grown in medium supplemented with Bock for the suppression of the amber codon. The suppression efficiency of amber and/or frameshift codons was measured by the amount of GFP produced. We expected that the better a plasmid combination worked, the higher the concentration of GFP would be. In 
Figure 4.31 a comparison of the modular genetic tool to the four plasmid system is shown. Additionally, a variant of the genetic tool was tested harboring a gene for MjYT_UCCU with a mismatch in the anticodon stem $\left(\mathrm{C}_{29}-\mathrm{A}_{44}\right.$ instead of $\mathrm{C}_{29}-\mathrm{G}_{44}$, pCLA109).

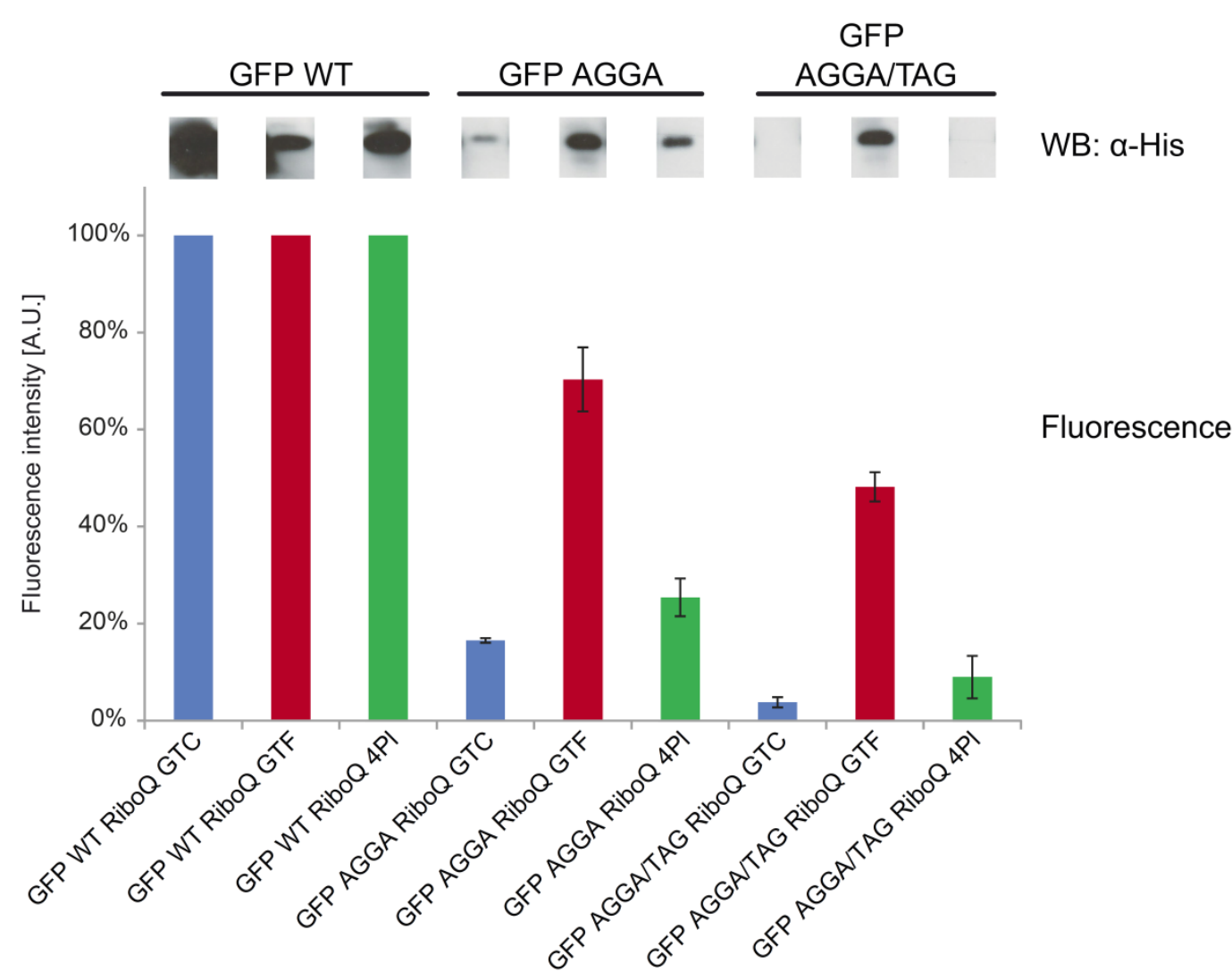

Figure 4.31: Comparison of the modular genetic tool to the four plasmid system.

The expression efficiencies of WT GFP (pCLA105), GFP with a frameshift codon (pCLA108) and GFP with a frameshift and an amber codon (pCLA107) were investigated via fluorescence measurement in a plate reader (lower panel; Ch. 3.2.2.12) and western blot (upper panel; Ch. 3.2.2.4). Three systems were compared: GTC = Genetic Tool Control (mismatch in MjYT_UCCU; pCLA109), GTF=Genetic Tool Final (pCLA95), $4 \mathrm{PI}=4$ Plasmid system (pCLA97 and pCLA98). All orthogonal mRNA translations were driven by ribo-Q1 (pCLA96). A final concentration of $1 \mathrm{mM}$ Bock was added to the medium. Two distinct samples were taken of each condition tested at different time points (GTC and GTF after 7 and $15 \mathrm{~h} ; 4 \mathrm{PI}$ after $72 \mathrm{~h}$ (pre-culture), 20 and $26.5 \mathrm{~h}$ (main culture) because they grew much slower than variant 1-cells), with one being normalized to $\mathrm{OD}_{600}$ of 1.5 for usage with western blots and one being normalized to $\mathrm{OD}_{600}$ of 0.5 for the detection of GFP in a plate reader (Ch. 3.2.2.12). For the western blot whole cell extracts were separated with SDS PAGE (Ch. 3.2.2.3) and blotted onto a PVDF membrane (Ch. 3.2.2.4). Anti-His-antibody was used as primary antibody. Fluorescence measurements were always performed in duplicates. The mean values of GFP WT were set to $100 \%$ for each system individually.

The employment of the modular genetic tool resulted in higher expression levels of GFP compared to the four plasmid system (Figure 4.31). Although the general translation efficiency was similar as shown for GFP WT, the decoding of an AGGA codon performed up to three fold more efficiently. The combination of an AGGA and a UAG codon yielded five times more protein. Results could be confirmed by western blot. Cells with the control mismatch tRNA seemed to be almost completely inactive. 
Using the modular genetic tool increased the expression level of the reporter protein GFP significantly. Beside this, we observed that the viability of cells transformed with the genetic tool, and thereby with one plasmid less than those cells transformed with the four plasmid system, was three to five times better (for details see Ch. 4.4.6). Concerning these findings the modular genetic tool already demonstrated a powerful tool that optimized the established system.

\subsubsection{The Combination of The Modular Genetic Tool and The Promoter Libraries}

The promoter libraries explained in Ch. 4.2, especially for the aaRS PyIS, already showed improvements for the incorporation of UAAs into proteins. The positive effect by using the modular genetic tool instead of the four plasmid system was illustrated in Ch. 4.4.3. Consequently, the next step was to test the combination of both optimization trials to obtain an even more efficient genetic tool with enhanced promoters to balance the simultaneous expression of both tRNA/aaRS pairs in the cell.

To this end, large scale preparative digests with 10 to $30 \mu \mathrm{g}$ of DNA, from backbone and insert vectors, were performed to cover the diversities of the distinct libraries. The same restriction enzymes described in Table 4.3 were used because the library plasmids were based upon the plasmids (pCLA72, pCLA86, pCLA79 and pCLA82) used to construct the modular genetic tool. To avoid contaminations with wild type promoters, the libraries were cloned into a fresh pCDF Duet-1 (pCLA91) vector instead of exchanging the existing WT versions on the final plasmid (pCLA95). The ligation and transformation efficiency was checked by dilution series for each cloning step.

First, we cloned the PyIS and PyIT promoter libraries into PCDF Duet. The transformations of E. coli $\mathrm{DH} 10 \mathrm{~B}$ via electroporation yielded with $10^{6}$ to $10^{7}$ different clones. However, the cloning of the PylT library revealed a strong background, defined by the number of colonies counted on agar plates with clones from the negative control (ligation of vector backbone and water instead of insert DNA). Since these background clones were thought to be due to re-ligation of the digested backbone vector, we presumed to eliminate them using a modified chloramphenicol reporter harboring the gene for the chloramphenicol acetyltransferase with an amber codon but no tRNA (pCLA110). That means clones should only be able to grow on $\mathrm{Cm}$ if both libraries were successfully cloned into the pCDF vector. Hence, electro competent cells containing the modified reporter were prepared and 
transformed with up to $10 \mu \mathrm{g}$ of the combined pCDF PyIS-PyIT library plasmids. Afterwards, cells were selected on chloramphenicol (Figure 4.32).

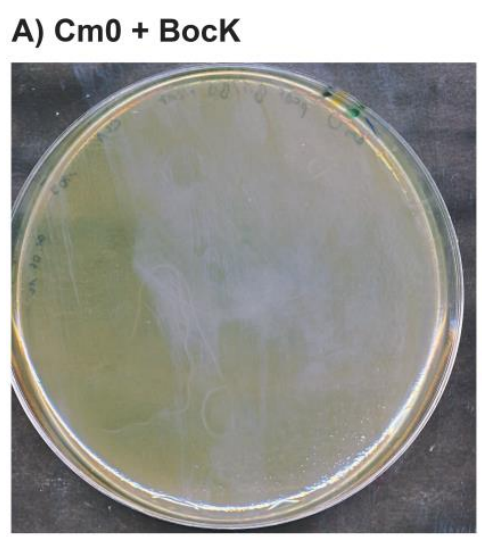

B) $\mathrm{Cm} 50+$ BocK

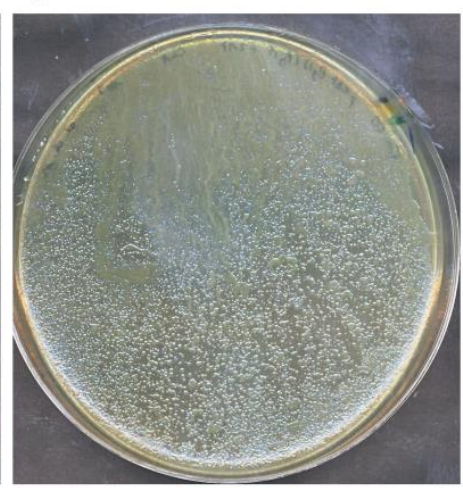

E) $\mathrm{Cm} 50$ - BocK

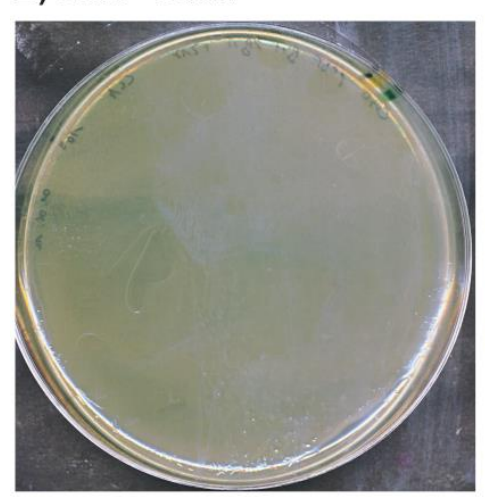

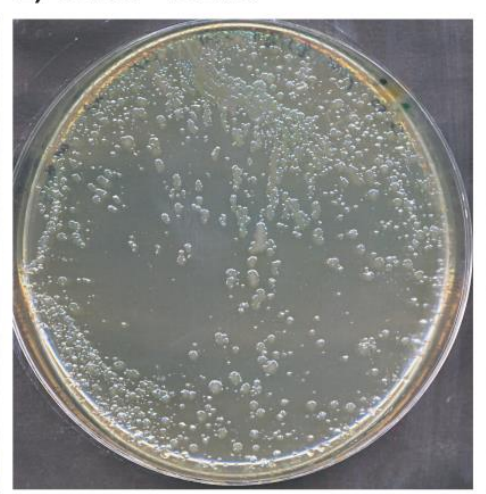

C) $\mathrm{Cm} 100+$ BocK

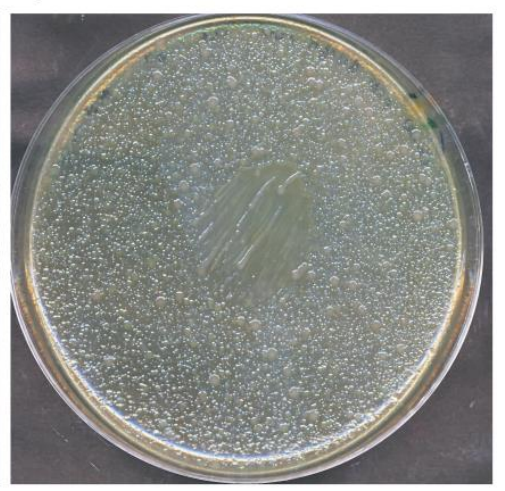

F) Cm100 - BocK

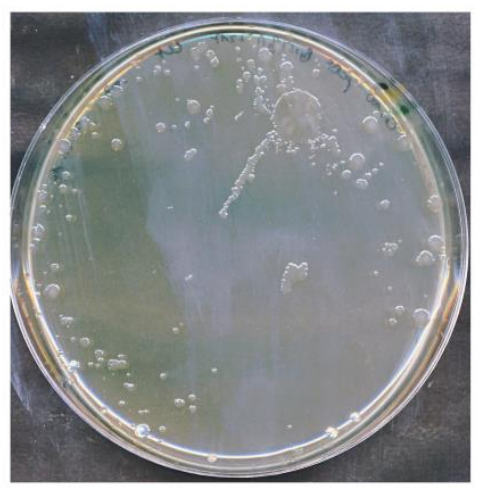

Figure 4.32: $\mathrm{Cm}$-Assay for the background reduction of combined PyIS and PyIT promoter library.

Cells harboring the delta tRNA Cm reporter (pCLA110) and the PCDF PyIS-PyIT promoter library plasmids were plated ( $2 \mathrm{~mL}$ cell culture with an $\mathrm{OD}_{600}$ of 2.6 each) on agar plates containing $\mathrm{Sm}$, Tet, increasing $\mathrm{Cm}$ and $1 \mathrm{mM}$ Bock ( $A$ to $\mathbf{C}$ ) or no Bock (D to $\mathbf{F}$ ).

Assuming that cells without any PylT did not survive the $\mathrm{Cm}$ assay, the background reduction for the combination of the PyIS and PyIT library conferred amino acid dependency, since only a fraction of cells survived without the UAA BocK, as depicted in Figure 4.32. The cells from the agar plate with a $\mathrm{Cm}$ concentration of $50 \mu \mathrm{g} / \mathrm{mL}$ and BocK were scraped off for plasmid isolation, as already described in Ch. 4.2.1. The purified pCDF PyIS-PyIT library plasmids were subsequently used for the cloning of the MjYRS_AGGA and MjYT_UCCU libraries. Both yielded, on average, $10^{6}$ different clones. Thus, all four libraries described in Ch. 4.2 were successfully cloned into pCDF Duet, confirmed by restriction digests in comparison to the wild type modular genetic tool (pCLA95; Figure 4.33). 


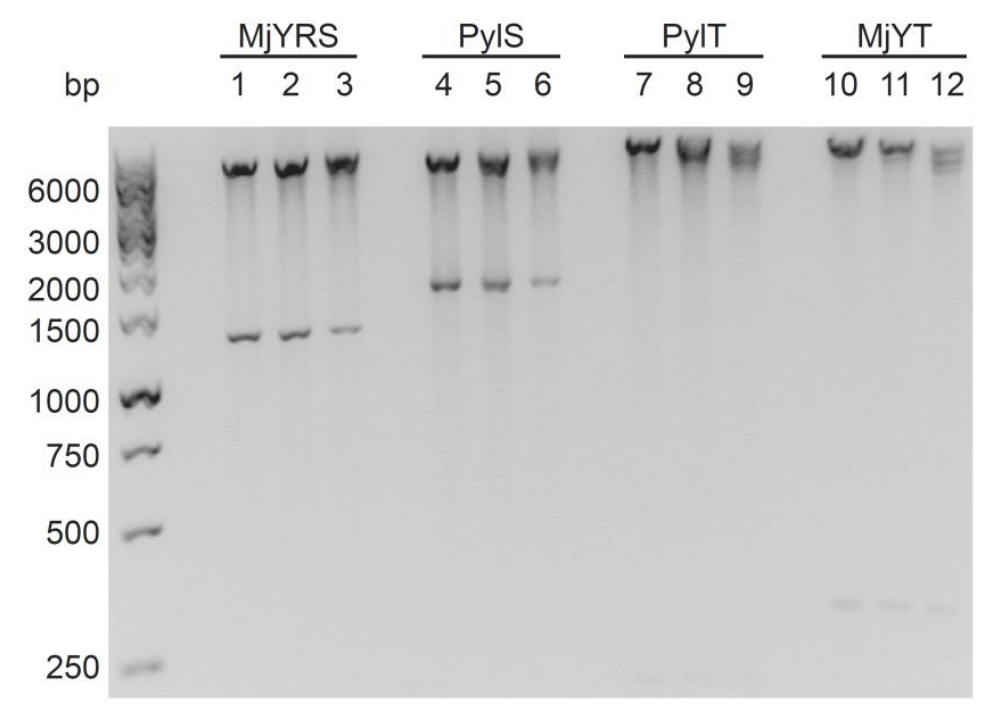

Figure 4.33: Restriction digests of the modular genetic tool with wild type and library promoters.

The final modular genetic tool with wild type glnS and Ipp promoters (pCLA95; digests 1, 4, 7 and 10) was compared to two sets of the tool containing the promoter libraries of all four components (sets differed in diversity only: set1 had $5 * 10^{5}$ clones (digests 2, 5, 8 and 11 ); set2 had $3 * 10^{6}$ clones (digests $3,6,9$ and 12). Enzymes and sizes: BamHI and Sall $(13=5618$ and 1206); Sall and Notl (4-6=5269 and 1555); Notl and Mfel (7-9 = 6612 and 212); Mfel and Xhol (10-12 = 6506 and 318).

The quantification of the suppression efficiency for the modular genetic library tool was performed by GFP reporter assay (Ch. 3.2.4.2), as previously described for the WT promoter version (Ch. 4.4.3). Therefore, previously prepared competent cells that held the plasmids pCLA96 and pCLA107 for the orthogonal ribosome ribo-Q1 and the ORBS GFP reporter comprising the frameshift codon AGGA and the amber codon UAG, respectively, were transformed with the pCDF PyIS-PyIT-MjYRS-MjYT library plasmids. The enormous variety of different promoter sequences made it impossible to investigate the GFP expression levels separately via plate reader or western blot. The method of choice used for this purpose was the fluorescence-activated cell sorting (FACS) which was established by the workgroup of Herzenberg ${ }^{[129]}$. FACS (Ch. 3.2.2.13) was performed at the MRC Laboratory of Molecular Biology, Cambridge. Cells transformed with plasmids pCLA96 and PCLA105, expressing GFP WT, were used as a positive control. In order to set a threshold for the suppression of the AGGA and TAG codon in the GFP gene (pCLA107) that had to be exceeded by more efficient library clones, cells were prepared that contained the modular genetic tool with wild type promoters (pCLA95) as a reference. The results of the FACS are shown in Figure 4.34 in summary. 
A) GFP WT

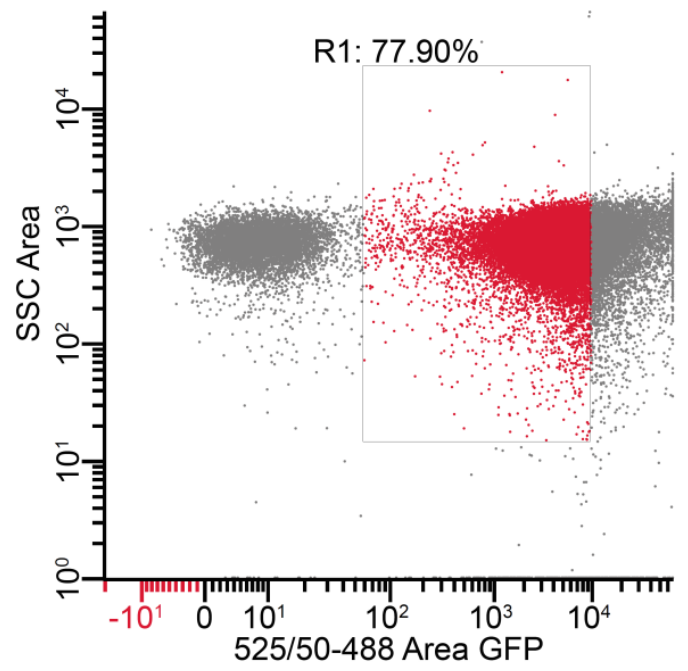

C) GFP TAG/AGGA using GT4L

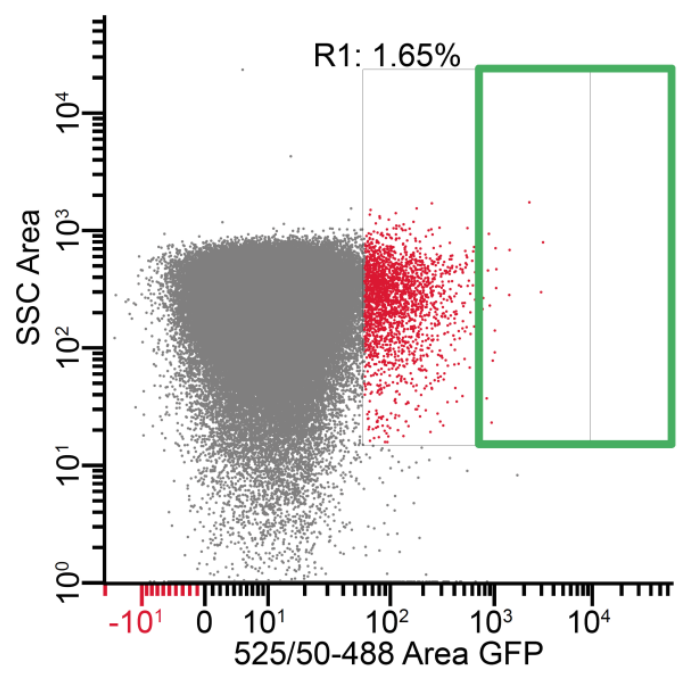

B) GFP TAG/AGGA using GTF

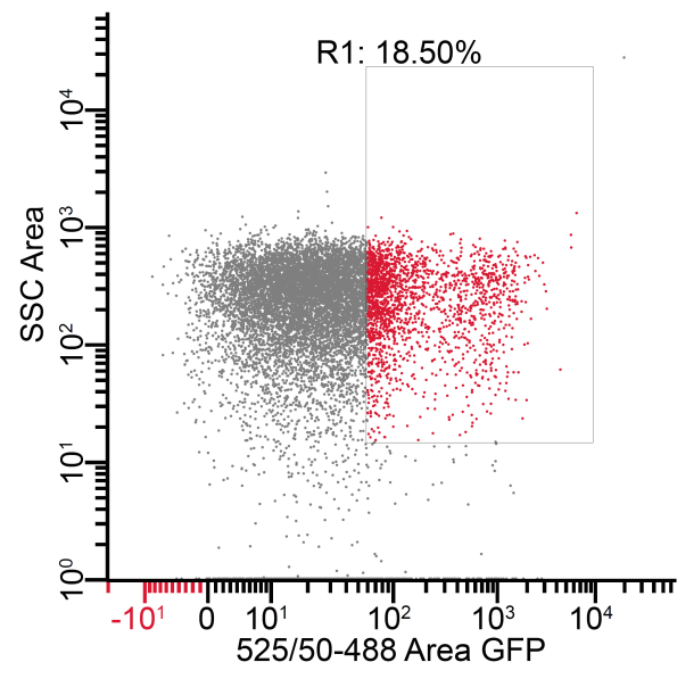

D) GFP TAG/AGGA using GT4L 2. round

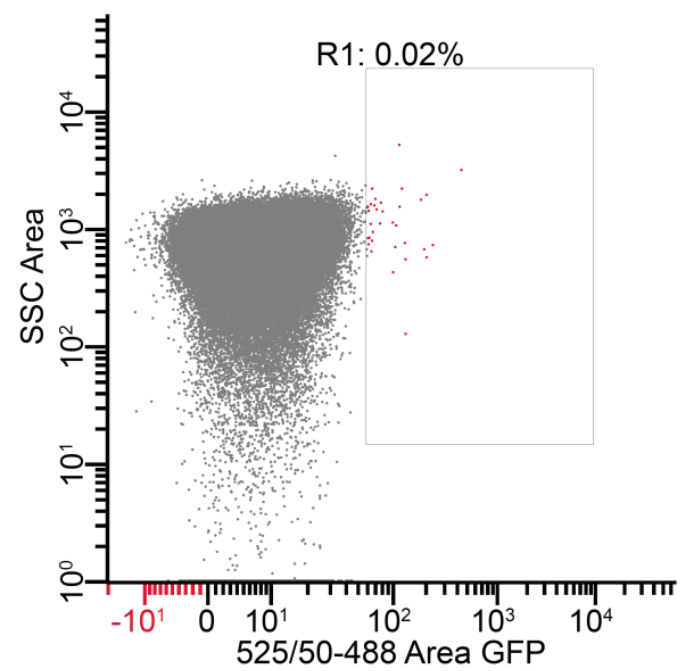

Figure 4.34: FACS to identify the best combination of all 4 libraries on the Genetic Tool at once (GT4L).

A) sfGFP WT ( $p C L A 105$ ) expression to set up the screening area. Positive fluorescence signals are found on the right side of the plot. Each dot illustrates a measurement of a single cell/event by the FACS machine. Cells/events chosen to be collected are found within the rectangle and colored red. R1 represents the relative amount of cells/events within the rectangle compared to the total amount of cells/events tested. B) sfGFP harboring a TAG and an AGGA codon in the gene (pCLA107) was expressed using the GTF (Genetic Tool Final (pCLA95) - standard glnS (aaRS)/Ipp (tRNAs) promoter) to identify maximum fluorescence/expression level using the standard system. C) Screening for high fluorescence signals (green rectangle) resulting from sfGFP expression using the GT4L plasmids. D) Second round of screening cells with GT4L plasmids using isolated cells from C). It seemed the machine sorted out mainly false positive ones, thus no improved GFP signals could be detected.

We did not observe clones from the four library plasmids that displayed enhanced GFP expression compared to the standard WT promoter genetic tool (pCLA95). In Figure 4.34-A the majority of the events, which ideally represent the fluorescence measurements of single cells, are arranged on the right side of the plot. This represents an intense fluorescence signal due to high expression levels of sfGFP. In order to exclude false positive events by virtue of more than one cell measured at the same time, the screening and 
collecting area (rectangles) was not set to the most right. This setting still included about $78 \%$ of all cells tested. In contrast, the expression of the GFP gene with an amber and a frameshift codon by the aid of the standard modular genetic tool (pCLA95) reduced the number of positive clones to roughly $19 \%$ (Figure 4.34-B). The majority of these clones was less active, or inactive, indicated by the shift to the left side of the plot. The employment of the four library plasmids (Figure 4.34-C) had an even more dramatic effect on the fluorescence intensities. A minority, of less than $2 \%$ of the cells tested, was active and located within the rectangle. To obtain the clones that were able to produce more GFP than those with the standard genetic tool, the screening area was shifted to the right (green rectangle). In the end, performing a second round of FACS with those collected cells (Figure 4.34-D) revealed no improvements.

\subsubsection{Alternative Strategies For Screening The Modular Genetic Library Tool}

Although FACS is an established method for sorting cells based on their fluorescent characteristic, it was not possible to find any better library composition that exceeded the performance of the standard modular genetic tool. Moreover, a lot of clones seemed to be completely inactive (Figure 4.34). Considering these facts, the search for alternative strategies to screen the four library plasmids led back to the already established chloramphenicol reporter system, which should eliminate inactive clones. To keep the expression system comparable to the GFP reporter used in the last chapters, we made a new $\mathrm{Cm}$ reporter including an orthogonal ribosomal binding site based on a reporter produced by Wang ${ }^{[67]}$ (pCLA111). This plasmid contained the gene for the CAT only but not for a tRNA. We exchanged the codons for T6 and D111 in the CAT gene for TAG and AGGA, respectively (pCLA112 and pCLA113).

Similar to the GFP reporter previously prepared competent cells harboring the ORBS CAT reporter plasmid ( $\mathrm{pCLA113)}$ ) and the orthogonal ribosome plasmid ( $\mathrm{pCLA96)}$ were transformed with the four library plasmids. Three library plasmid variants, missing the library for MjYT, and the standard genetic tool (pCLA95) were transformed as negative and positive control, respectively. In addition, we investigated differences between a CAT gene with TAG alone and together with the AGGA codon. After a low stringency selection on chloramphenicol colonies were counted to determine the reliability of the reporter (Table 4.4). 
Table 4.4: Cm-Assay for the sorting of the four library plasmids.

Competent cells harboring plasmids for the orthogonal ribosome ribo-Q1 (pCLA96) and either the ORBS CAT reporter with T6TAG alone ( $p$ CLA112) or together with D111AGGA (pCLA113) were transformed with the standard modular genetic tool (pCLA95; GTF), the four library plasmids (GT4L) or the three library variants (GT3L; MjYT library missing). In case of the library plasmids up to $5^{*} 10^{7}$ cells were plated and up to 1000 cells were plated for the standard genetic tool on agar plates containing Sm, Tet, increasing $\mathrm{Cm}$ in the absence and presence of $1 \mathrm{mM}$ Bock. Plates were incubated at $37 \mathrm{C}$ for two days due to slow growth of the cells. $\mathrm{MSC}=$ "many single colonies"

\begin{tabular}{|c|c|c|c|c|c|c|c|}
\hline \multirow[b]{2}{*}{$\begin{array}{c}\mathrm{Cm} \\
{[\mu \mathrm{g} / \mathrm{mL}]}\end{array}$} & \multicolumn{4}{|c|}{ GTF (standard) } & \multicolumn{2}{|c|}{ GT4L } & \multirow{2}{*}{$\begin{array}{c}\text { GT3L } \\
\text { T6/D111 } \\
\text { +BocK }\end{array}$} \\
\hline & $\begin{array}{c}\text { T6 } \\
+ \text { +BocK }\end{array}$ & $\begin{array}{c}\text { T6 } \\
\text {-Bock }\end{array}$ & $\begin{array}{l}\text { T6/D111 } \\
\text { +BocK }\end{array}$ & $\begin{array}{l}\text { T6/D111 } \\
\text {-BocK }\end{array}$ & $\begin{array}{l}\text { T6/D111 } \\
\text { +BocK }\end{array}$ & $\begin{array}{l}\text { T6/D111 } \\
\text {-BocK }\end{array}$ & \\
\hline 0 & $\approx 960$ & 872 & 1088 & $\approx 864$ & Lawn & Lawn & Lawn \\
\hline 10 & $\approx 788$ & 0 & 507 & 0 & Lawn & Lawn & 37 \\
\hline 25 & 420 & 0 & 30 & 0 & MSC & MSC & 12 \\
\hline 50 & 2 & 0 & 1 & 0 & $\approx 1000$ & $>1000$ & 3 \\
\hline 75 & 0 & 0 & 0 & 0 & 78 & $\approx 500$ & 1 \\
\hline 100 & 0 & 0 & 0 & 0 & 25 & 75 & 0 \\
\hline 150 & 0 & 0 & 0 & 0 & 9 & 8 & 0 \\
\hline
\end{tabular}

The orthogonal CAT reporters (pCLA112 and pCLA113) both worked as demonstrated in Table 4.4 by the quantity of colonies for the standard modular genetic tool. Cells were able to survive treatment with $\mathrm{Cm}$ only in the presence of the UAA BocK. The additional AGGA codon in the CAT gene reduced the growth of the bacteria 10-fold concerning the plates with $25 \mu \mathrm{g} / \mathrm{mL} \mathrm{Cm}$. The reporter itself seemed not to be that strong than the pREP reporters used earlier since the cutoff was around $50 \mu \mathrm{g} / \mathrm{mL}$ instead of 250 to $400 \mu \mathrm{g} / \mathrm{mL}$. The majority of cells lacking the MjYT library died upon $\mathrm{Cm}$ treatment, confirming that no other endogenous tRNA/aaRS pairs suppressed the AGGA codon. Considering the four library plasmids, no dependence on the UAA could be observed. Approximately the same numbers of colonies survived on plates with and without Bock.

These findings led us to the assumption that even if inactive clones were eliminated using the chloramphenicol reporter assay, collateral mutations during the mutagenesis of the promoter regions could have caused the use of natural amino acids by the synthetases, 
especially PylS. Therefore, the four library plasmids from the agar plate with $25 \mu \mathrm{g} / \mathrm{mL} \mathrm{Cm}$ and Bock were separated from the orthogonal CAT reporter and ribosome plasmids by destroying the unwanted plasmids with the restriction enzyme Sphl and removing them by transformation of E. coli $\mathrm{DH} 10 \mathrm{~B}$ with the intact library plasmids. In order to get rid of PylS synthetases which used natural amino acids as substrate to charge PyIT, a negative selection was performed based on suppression of amber nonsense mutations in the barnase gene as described by Wang and Schultz ${ }^{[146]}$. Without its inhibitor, barstar, it is lethal to cells ${ }^{[46]}$. That means, synthetases that are using natural amino acids in the absence of the UAA will finally suppress the amber codons in the barnase gene and mediate the cell death. Cells with synthetases that are still orthogonal and only using UAAs will be filtered (Ch. 3.2.4.3). To this end, electro competent cells containing the plasmid with the amber mutated barnase gene ( $\mathrm{pCLA114}$ ) were transformed with the purified four library plasmids as well as the standard modular genetic tool (pCLA95) as a comparison. A total number of $5^{*} 10^{7}$ transformed cells were plated on agar plates with the appropriate antibiotics in the absence and presence of $0.002 \%$ arabinose, to induce the expression of the barnase gene, and Bock, as a negative control.

We observed nearly the same growth behavior on all plates regardless of conditions (data not shown). A small tendency of less growth in the presence of UAA BocK could be stated. Thus, we were not able to solve the problem of the amino acid independent suppression of amber codons by using the barnase assay.

A second alternative strategy was then used. This method relied on the direct monitoring of GFP expression via western blot. 96 colonies were picked for this purpose from the remaining agar plates of the ORBS CAT reporter assay mentioned at the beginning of this chapter - including two clones for four library plasmids from plates without Bock and two clones from the three library plasmid plate. These 96 single clones were put again on agar plates with increasing $\mathrm{Cm}$ for re-phenotyping. Finally, a selection of 20 clones for the four library plasmids including the two clones from plates without Bock and the two clones for the three library plasmids were further analyzed. Isolated library plasmids were investigated by restriction digest (not shown) and sequencing. The digest revealed that only half of the clones had the correct banding pattern, indicating the absence of the MjYRS library. However, the sequencing confirmed that the majority of mutations were located in the promoter regions, as desired. Next, similar to the procedure described in Ch. 4.4.3 competent cells were prepared that held plasmids for the orthogonal ribosome ribo-Q1 (pCLA96) and the ORBS GFP reporter comprising the frameshift codon AGGA and the amber 
codon UAG (pCLA107), respectively, were transformed with all 22 plasmids to perform the GFP reporter assay (Ch. 3.2.4.2). The standard modular genetic tool (pCLA95) served as a reference for the GFP expression level. The comparative western blot is shown in Figure 4.35 .

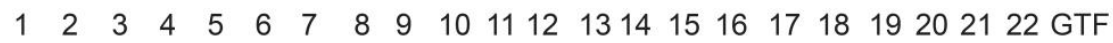

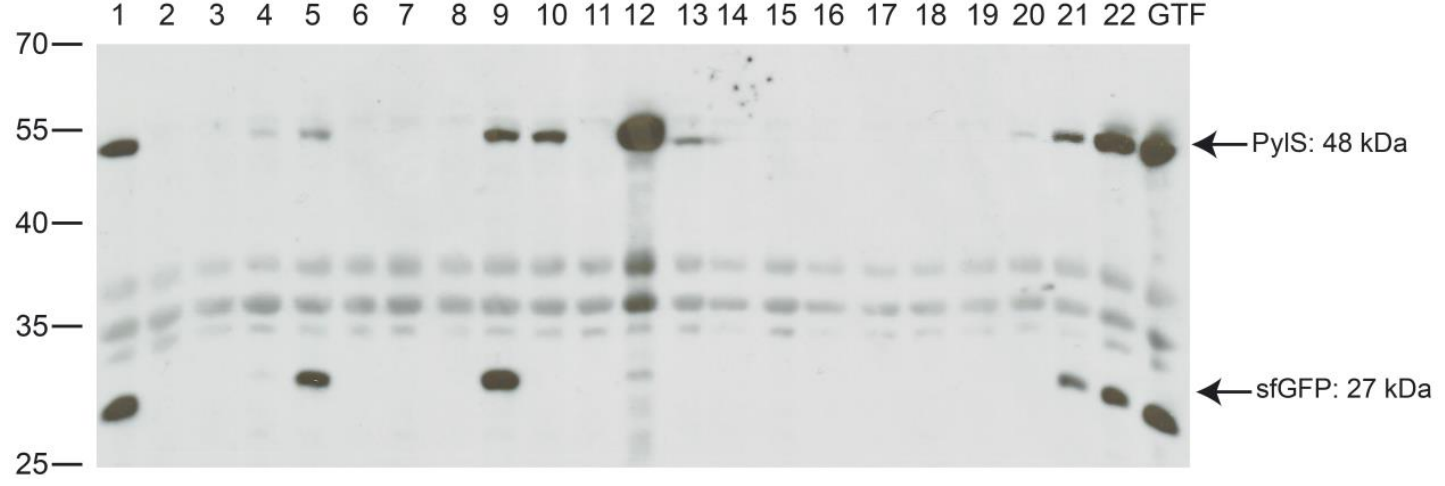

Figure 4.35: Comparison of the modular genetic tool (GTF) to single four library plasmid clones.

The expression efficiencies of GFP with a frameshift and an amber codon (pCLA107) were investigated via western blot (Ch. 3.2.2.4). The modular genetic tool (GTF; pCLA95) was compared to 22 different library clones. Clone number 5 and 15 originated from four library plasmid plates without 1 mM Bock. Clone number 10 and 20 were picked from three library plasmid plates. The rest of the clones came from four library plasmid agar plates with BocK and different concentrations of $\mathrm{Cm}$. All orthogonal mRNA translations were driven by ribo-Q1 (pCLA96) with $1 \mathrm{mM}$ of BocK to suppress the UAG codon. Samples taken the end were normalized to $\mathrm{OD}_{600}$ of 1.5. For the western blot whole cell extracts were separated with SDS PAGE (Ch. 3.2.2.3) and blotted onto a PVDF membrane (Ch. 3.2.2.4). Anti-His-antibody was used as primary antibody.

Figure 4.35 clearly shows that most of the selected clones did not express any sfGFP. Those clones that did were, in the end, not better than the wild type modular genetic tool (GTF; pCLA95). For clone number 10 and 20 no GFP was expected due to the lack of MjYT. We noticed again the correlation of produced PyIS and protein of interest observed in earlier chapters (Ch. 4.2.1 and 4.3), with more PyIS leading to more GFP in this case. However, it seemed that a strong expression level of PylS caused a negative effect as depicted by clone number 12. This clone revealed the highest levels of expressed PylS but only a very small amount of GFP.

In summary, it was not possible to isolate a clone out of the four component library plasmids that exceeded the standard modular genetic tool's suppression efficiency for a UAG and AGGA codon at the same time. 


\subsubsection{The Combination of The Modular Genetic Tool And Defined (Standard) Promoters}

Since the combination of the modular genetic tool and the promoter libraries brought no significant improvements in the end (Ch. 4.4.4 and 4.4.5), we thought to incorporate the defined standard promoters introduced in Ch. 4.3 into the tool. The most promising ones were selected, i.e., $\mathrm{P}_{\mathrm{lib3E}}, \mathrm{P}_{\text {con }}$ and $\mathrm{P}_{\mathrm{lac}}$, and cloned into pCLA95 exchanging the gInS promoter thereby.

The effect of the promoter exchanges was tested with the GFP reporter assay in the same way as described in Ch. 4.4.3. GFP expression levels were determined by western blots (Ch. 3.2.2.4) and by in-cell fluorescence of sfGFP in a plate reader (Ch. 3.2.2.12). In a first attempt we found a strong degradation of PyIS on western blot (not shown). To exclude that bands at the height of GFP were due to PyIS degradation, background controls with the synthetase containing pCDF vectors alone were also analyzed (Figure 4.36).

Both the western blot and the fluorescence measurements in Figure 4.36 revealed that the change of the glnS wild type promoter to $\mathrm{P}_{\text {con }}$ and $\mathrm{P}_{\text {lac }}$ drastically increased the GFP expression as well for the amber codon only version as for the combined amber and frameshift codon version. In detail, $\mathrm{P}_{\text {con }}$ was at least 1.5 to 2 -fold better and $\mathrm{P}_{\text {lac }} 2.5$ to 3 -fold compared to the standard promoter in terms of fluorescence measured (Figure 4.36-B). Interestingly, $\mathrm{P}_{\text {lib3E }}$, which was shown to be significantly stronger than $\mathrm{P}_{\text {std }}$ in earlier chapters (Ch. 4.2.1), was found to be weaker after changing the plasmid from pBK to pCDF. It could be excluded that signals at the height of GFP detected on the western blot in Figure 4.36-A relied on PylS degradation because no comparable signals were found in the samples with Pyls alone.

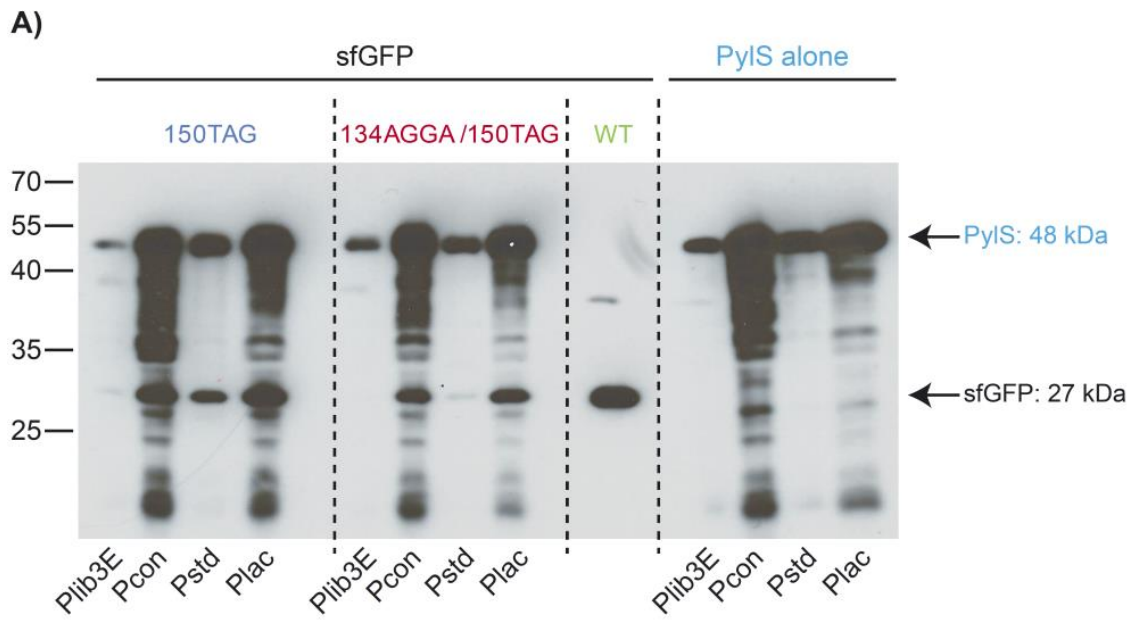


B)

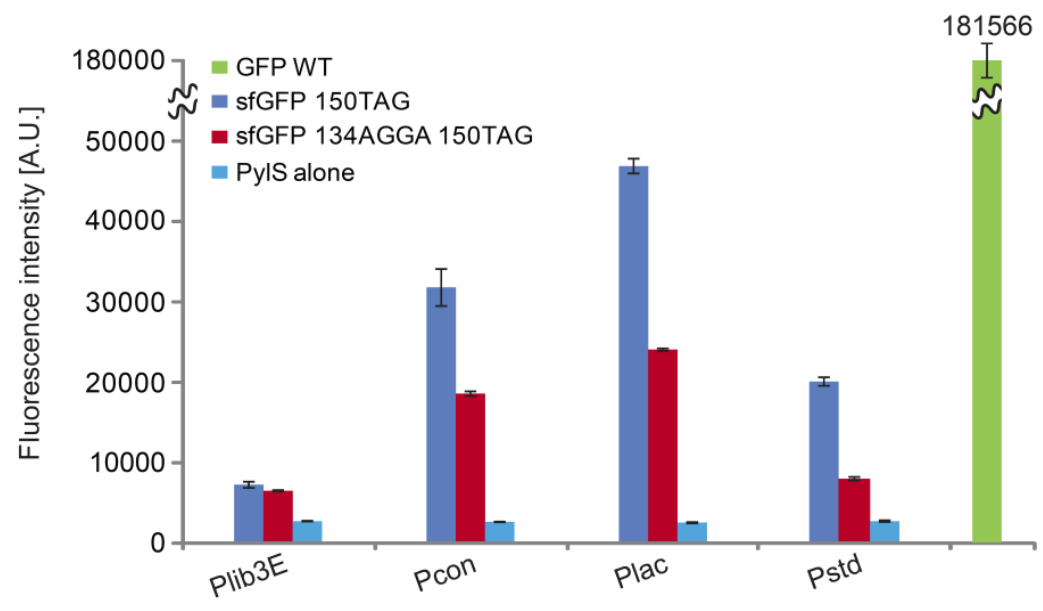

Figure 4.36: Comparison of the standard modular genetic tool to variants with defined promoters.

The expression efficiencies of WT GFP (pCLA105), GFP with an amber codon (pCLA106) and GFP with a frameshift and an amber codon ( $\mathrm{CLCA107)}$ were investigated A) via western blot (Ch. 3.2.2.4) and B) via fluorescence measurement in a plate reader (Ch. 3.2.2.12). Four variants of the modular genetic tool were compared, only differing in the promoter in front of the pylS gene: $\mathrm{P}_{\text {std }}=$ wild type $g$ InS promoter on pCLA95; $\mathrm{P}_{\text {lib3E }}=$ PylS library promoter $3 \mathrm{E}$ on pCLA115; $\mathrm{P}_{\text {con }}=$ consensus promoter on $\mathrm{pCLA116;} \mathrm{P}_{\text {lac }}=$ lac promoter on pCLA117. All orthogonal mRNA translations were driven by ribo-Q1 (pCLA96). E. coli DH10B transformed with pCLA95, pCLA115, pCLA116 and pCLA117 only, were used as a background control. A final concentration of $1 \mathrm{mM}$ Bock was added to the growth medium for the suppression of the amber codon. In case of the lac promoter, expression was induced with $1 \mathrm{mM}$ IPTG Two distinct samples were taken of each condition tested, with one being normalized to $\mathrm{OD}_{600}$ of 1.5 for usage with western blots and one being normalized to $\mathrm{OD}_{600}$ of 0.5 for the detection of GFP in a plate reader (Ch. 3.2.2.12). For the western blot whole cell extracts were separated with SDS PAGE (10 $\mu \mathrm{L}$ loaded for each sample, except for GFP WT with $5 \mu \mathrm{L} ;$ Ch. 3.2.2.3) and blotted onto a PVDF membrane (Ch. 3.2.2.4). Anti-His-antibody was used as primary antibody. Fluorescence measurements were always performed in duplicates.

As a proof of principle, to demonstrate that the effects on the suppression efficiencies observed for GFP due to the changes in the PyIS promoter were also valid for other proteins, we did a final experiment comparing $\mathrm{P}_{\text {std }}, \mathrm{P}_{\text {con, }} \mathrm{P}_{\text {lac }}$ and the previously used four plasmid system (with standard gInS and Ipp promoters). The GFP reporter plasmid was exchanged with a variant of its original vector pO-gst-malE ${ }^{[67]}$ which harbored an AGGA codon at position Y17 and a TAG codon at position N234 in the malE gene (pCLA118). We kept the conditions for the expression the same, except for the scale. A $50 \mathrm{~mL}$ small scale expression was performed and the GST-MBP fusion proteins were purified from cell lysates (Ch. 3.2.2.6) to visualize differences in protein levels by SDS-PAGE instead of a western blot (Figure 4.37). 


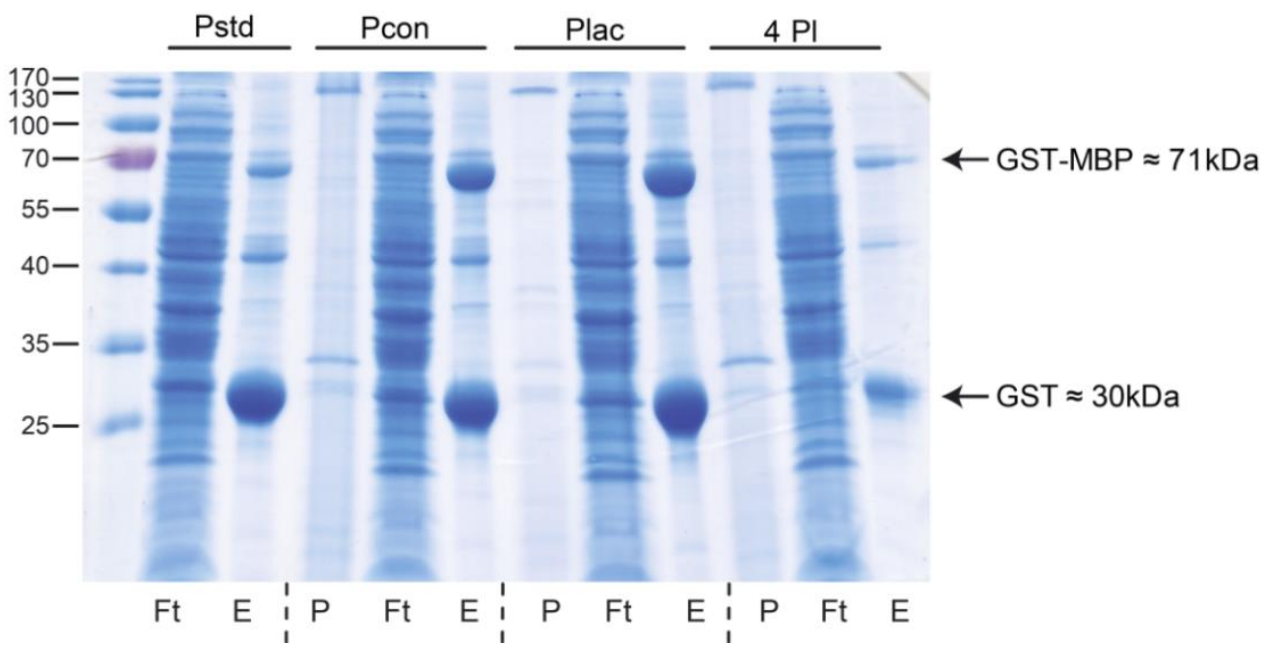

Figure 4.37: Small scale expression and purification of GST-MBP comparing different genetic tool variants and the 4 plasmid system.

The expression efficiency of gst-malE harboring an AGGA codon at position Y17 and a TAG codon at position N234 in the malE gene (pCLA118) was compared in four different systems: $P_{\text {std }}=$ wild type glnS promoter on

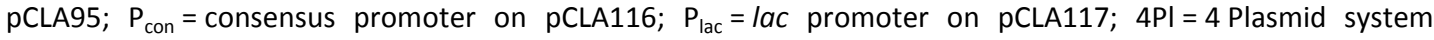
(pCLA97 and pCLA98). All orthogonal mRNA translations were driven by ribo-Q1 (pCLA96). A final concentration of $1 \mathrm{mM}$ Bock was added to the growth medium for the suppression of the amber codon. Expression was performed in a $50 \mathrm{~mL}$ scale and cell densities normalized by $\mathrm{OD}_{600}$ after pelleting. GST-MBP fusion proteins were purified from cell lysates using glutathione sepharose beads (Ch. 3.2.2.6).Proteins were separated with SDS PAGE (15 $\mu \mathrm{L}$ loaded for each sample; Ch. 3.2.2.3). Ft = Flowthrough; $E=$ Elution; $P=$ Pellet.

The small scale expression and purification experiment shown in Figure 4.37 validated our previous experiments. The four plasmid system offered the lowest expression and thereby suppression efficiency of all the conditions tested. The use of the standard modular genetic tool increased the full-length GST-MBP level substantially. However, when the glnS promoter of the pylS gene is exchanged for $\mathrm{P}_{\text {con }}$ or $\mathrm{P}_{\text {lac }}$ the yield of full-length GST-MBP was again boosted enormously.

Beside the fact that cells with the four plasmid system had the lowest expression efficiencies, another observation already showed that their growth rate was at least three to five times lower compared to the three plasmid system with the modular genetic tool variants (Ch. 4.4.3). To visualize these differences we made a growth curve with the three modular genetic tool variants $\mathrm{P}_{\text {std }}, \mathrm{P}_{\text {con, }}, \mathrm{P}_{\text {lac }}$ and the four plasmid system. For this reason, competent cells that harbored the plasmids for the orthogonal ribosome ribo-Q1 (pCLA96) and the ORBS GFP reporter, comprising the frameshift codon AGGA and the amber codon UAG (pCLA107), respectively, were transformed with the appropriate plasmids in order to monitor the growth rate and the production of GFP, simultaneously, by using a plate reader. The results of the growth assay and the fluorescence measurements are plotted in one graph (Figure 4.38). 


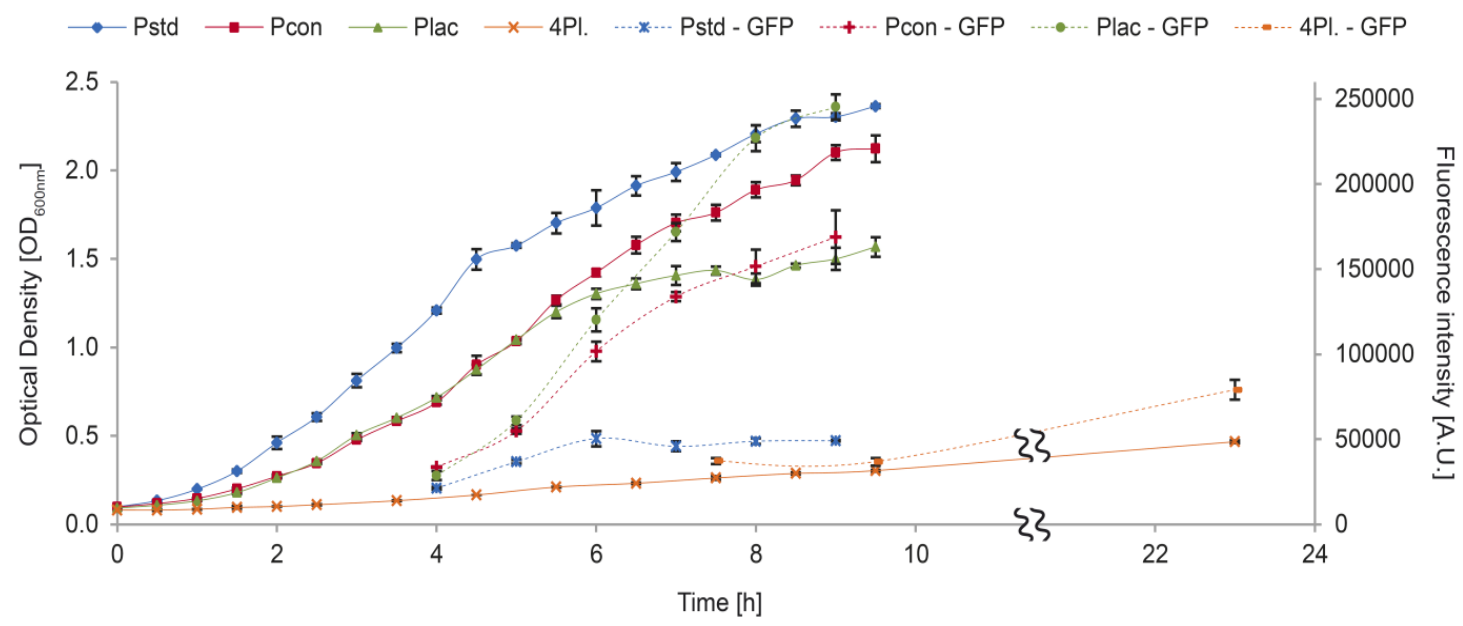

Figure 4.38: Growth curve and GFP fluorescence measurement comparing different genetic tool variants and the 4 plasmid system.

The growth rates and the expression efficiencies of sfGFP harboring an AGGA codon at position D134 and a UAG codon at position N150 (pCLA107) was compared in four different systems: $\mathrm{P}_{\text {std }}=$ wild type glnS promoter on

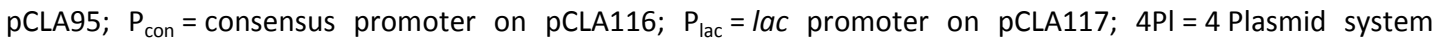
(pCLA97 and pCLA98). All orthogonal mRNA translations were driven by ribo-Q1 (pCLA96). Expression was performed in a $50 \mathrm{~mL}$ scale. Full-length GFP expression was initiated by the addition of the UAA BocK to a final concentration of $1 \mathrm{mM}$ after 3 hours for $\mathrm{P}_{\text {std }}, \mathrm{P}_{\text {con }}$ and $\mathrm{P}_{\mathrm{lac}}$ and after 3.5 hours for $4 \mathrm{PI}$ due to the slow growth. $\mathrm{A}$ final concentration of $1 \mathrm{mM}$ IPTG was supplemented for $P_{\mathrm{lac}}$ as well. Samples for fluorescence measurements of $P_{\text {std }}, P_{\text {con }}$ and $P_{\text {lac }}$ were taken every hour upon UAA addition corresponding to 4, 5, 6, 7, 8 and 9 hours and normalized to $\mathrm{OD}_{600}$ of 0.5 for the detection of GFP in a plate reader (Ch. 3.2.2.12). Samples for 4PI were taken after 4, 6 and 20 hours upon UAA addition corresponding to 7.5, 9.5 and 23.5 hours. $O_{600}$ was measured every 30 min for $P_{\text {std }}, P_{\text {con }}$ and $P_{\text {lac }}$ over a period of 9.5 hours. $O D_{600}$ for $4 \mathrm{PI}$ was measured every 30 min for the first 2.5 hours, every $60 \mathrm{~min}$ for the next 7 hours and finally one sample was taken after overnight incubation at 23.5 hours. All samples were taken in triplicates from three individual cultures.

The growth assay depicted in Figure 4.38 confirmed the observations made before. The viability of cells using the four plasmid system is several magnitudes lower than the cells with one of the three plasmid systems $P_{\text {std }}, P_{\text {con }}$ or $P_{\text {lac. }}$. The fluorescence intensity was indeed higher than for $\mathrm{P}_{\text {std }}$ but this was only achieved after overnight incubation. When comparing the three plasmid variants, $P_{\text {std }}$ had the fastest growth rates but also the lowest fluorescence intensities with a maximum already reached after 2 hours. Cells with the $P_{\text {con }}$ modular genetic tool were almost as viable as $\mathrm{P}_{\text {std, }}$ with a medium to high expression of GFP. $P_{\text {lac }}$ mediated a medium growth rate but produced, by far, the highest fluorescence intensities.To summarize, the use of the modular genetic tool improved the suppression efficiencies of amber and frameshift codons in protein expressions performed with the aid of the orthogonal ribosome ribo-Q1. The introduction of $\mathrm{P}_{\text {con }}$ and $\mathrm{P}_{\text {lac }}$ further increased this effect, with having one constitutively active and one inducible promoter for different purposes. Furthermore, the viability of the cells could be drastically enhanced by the reduction of plasmids from four to three, leading to better results in shorter expression times. 


\section{Applications And Side Projects}

\subsection{Evolution of A Quadruplet Decoding MjAzFRS}

A large variety of UAAs with different functionalities can be incorporated into proteins facilitated by the expansion of the genetic code. All previous experiments described in Ch. 4.1 to 4.4 were performed using the efficient substrate analogue Bock ${ }^{[169]}$ and/or tyrosine. Both amino acids do not provide any special functions because the Boc-protected lysine is not useful for further modifications and the tyrosine can also be incorporated using endogenous codons and tRNA/aaRS pairs. In contrast, aryl-azide, a photocrosslinking agent that was used first in a biological context by Fleet and colleagues who photochemically labeled antibodies with hapten ${ }^{[170]}$, represents one notably interesting group for incorporation into proteins. Furthermore, this group has been used to irreversibly inactivate enzymes ${ }^{[171]}$ and to probe protein-protein interactions ${ }^{[172,173]}$.

Chin and others enabled the site-specific incorporation of the azide containing UAA $p$-azido-L-phenylalanine (AzF) in good yield with high fidelity by evolving the M. jannaschii tyrosyl-tRNA synthetase (MjYRS) to an orthogonal synthetase that incorporates AzF $(\mathrm{MjAzFRS})^{[80]}$. Once incorporated into proteins the azide can be easily modified via bioorthogonal "click chemistry" even in cells and living organisms ${ }^{[114]}$. Whereas the paradigm of all click reactions, the $\mathrm{Cu}(\mathrm{I})$-catalyzed cycloaddition between an azide and a terminal alkyne ${ }^{[115]}$, is problematic due to its requirement for the cytotoxic copper ${ }^{[42]}$, the $\mathrm{Cu}(\mathrm{I})$-free alternative with cyclooctyne derivatives employs ring strain for alkyne activation $^{[116,117]}$. Chin and co-workers encoded AzF in combination with N6-[(2-propynyloxy)carbonyl]-L-lysine (CAK) in GST-calmodulin forming a redox-insensitive, crosslink by bioorthogonal cycloaddition that can be used to specifically constrain protein structure on the nanometer scale ${ }^{[78]}$. The Ebright laboratory coupled fluorescent probes by Staudinger ligation to genetically encoded AzF to investigate the opening and closing of the bacterial RNA polymerase clamp ${ }^{[118]}$.

The original tRNA/aaRS pair was designed to incorporate AzF in response to the amber codon UAG. In order to combine the M. jannaschii derived pair with similar amber decoding M. barkeri tRNA/aaRS pairs on the modular genetic tool we needed to change the codon specificity. In collaboration with the bachelor students Miguel Sánchez ${ }^{[174]}$ and Julia Motz ${ }^{[175]}$ the tRNA anticodon recognizing region of MjAzFRS was evolved to accept the expanded anticodon of the MjYT_UCCU tRNA as described by Neumann et a [ $^{[78,79]}$. 
To this end, the open reading frame (ORF) of pBK PyIS (pCLA1) was replaced by MjAzFRS (pCLA119) and subsequently used to create a library of $3 * 10^{6}$ mutant clones by randomizing the codons for residues Y230, C231, P232, F261, H283 and D286 (on pCLA120) in the tRNA anticodon recognizing region.

We analyzed 96 clones after an AzF dependent low stringency selection on chloramphenicol via broad range $\mathrm{Cm}$ reporter assay ( 0 to $500 \mu \mathrm{g} / \mathrm{mL}$ ). The 20 most promising clones (pCLA121 to pCLA140) were transferred to new $\mathrm{Cm}$ containing agar plates for rephenotyping (Figure 5.1) and sequenced to monitor codon changes (Table 5.1).

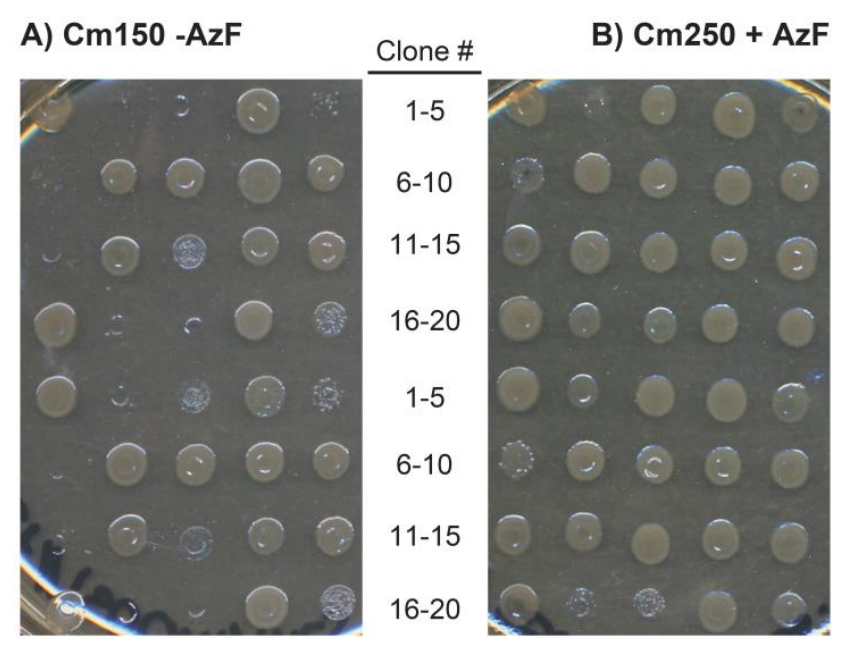

Figure 5.1: $\mathbf{C m}$-Assay with the 20 best clones from the MjAzFRS library.

Cells were plated on agar plates containing Kan, Tet, increasing $\mathrm{Cm}$ and $1 \mathrm{mM}$ AzF (B) or no AzF (A). A selection of two plates is shown only. Clones were plated in duplicates. Numbers ascend from left to right.

The clones with number 2, 3, 5, 6, 11, 17 and 18 (marked orange in Table 5.1) showed a growth clearly dependent on the presence of the amino acid AzF allowing them to survive a chloramphenicol concentration up to $250 \mu \mathrm{g} / \mathrm{mL}$. Without AzF they were not able to resist $\mathrm{Cm}$ at concentrations higher than $150 \mu \mathrm{g} / \mathrm{mL}$. The other clones grew on plates with a high $\mathrm{Cm}$ concentration even in the absence of AzF.

The amino acid sequence of the selected 20 clones revealed the common mutations Y230K, C231K and P232K, but divergent mutations at positions F261, H283 and D286. This observation was also made by Neumann and colleagues ${ }^{[78]}$ who suggested that amino acids 230, 231 and 232 grant affinity and specificity for the anticodon, and that 261, 283 and 286 may link the identity of the anticodon to the amino acid identity. 
Table 5.1: Amino acid sequence of the 20 best clones from the MjAzFRS library.

The mutated six amino acids are compared to the wild type amino acid sequence. Clones that showed an unnatural amino acid $(\mathrm{AzF})$ dependent growth on $\mathrm{Cm}$ containing agar plates are marked in orange.

\begin{tabular}{|c|c|c|c|c|c|c|c|}
\hline & & & & $\mathrm{Am}$ & 0 acid & & \\
\hline & WT & Y230 & C231 & P232 & F261 & $\mathrm{H} 283$ & D286 \\
\hline & 1 & K & K & $\mathrm{K}$ & $\mathrm{T}$ & G & $C$ \\
\hline & 2 & $P$ & $P$ & $A$ & $M$ & $\mathrm{~F}$ & $A$ \\
\hline & 3 & $Y$ & C & $P$ & G & G & A \\
\hline & 4 & K & K & $M$ & $\mathrm{~T}$ & G & $\mathrm{L}$ \\
\hline & 5 & $\mathrm{R}$ & K & $E$ & $\mathrm{~F}$ & $S$ & W \\
\hline & 6 & $\mathrm{~K}$ & K & $K$ & $P$ & $R$ & $Y$ \\
\hline & 7 & K & K & $\mathrm{K}$ & $\mathrm{F}$ & $\mathrm{L}$ & $Q$ \\
\hline & 8 & K & K & $\mathrm{K}$ & $\mathrm{F}$ & $S$ & $\mathrm{~F}$ \\
\hline & 9 & $\mathrm{~T}$ & $P$ & $P$ & $Q$ & G & G \\
\hline \# & 10 & K & K & $\mathrm{K}$ & $S$ & $P$ & $\mathrm{~S}$ \\
\hline 흠 & 11 & $\mathrm{R}$ & A & $\mathrm{H}$ & $\mathrm{F}$ & V & $M$ \\
\hline & 12 & $P$ & $\mathrm{~K}$ & $\mathrm{~L}$ & $S$ & G & $A$ \\
\hline & 13 & $\mathrm{~K}$ & $\mathrm{~K}$ & $\mathrm{~K}$ & G & $A$ & $\mathrm{~T}$ \\
\hline & 14 & $\mathrm{R}$ & $\mathrm{K}$ & $\mathrm{R}$ & $\mathrm{F}$ & $P$ & $\mathrm{H}$ \\
\hline & 15 & $\mathrm{~K}$ & K & $\mathrm{K}$ & $\mathrm{F}$ & A & $\mathrm{T}$ \\
\hline & 16 & $\mathrm{~K}$ & K & $\mathrm{K}$ & w & G & $Y$ \\
\hline & 17 & $\mathrm{~K}$ & $\mathrm{~K}$ & $\mathrm{~K}$ & W & G & $Y$ \\
\hline & 18 & $K$ & K & $K$ & $\mathrm{~F}$ & frameshift & @ K280 \\
\hline & 19 & K & K & $\mathrm{K}$ & $S$ & $P$ & $Y$ \\
\hline & 20 & K & $\mathrm{K}$ & $\mathrm{K}$ & $S$ & $P$ & $Y$ \\
\hline
\end{tabular}

To confirm the ability of the synthetases to suppress AGGA codons by charging the corresponding tRNA with AzF, we performed test expressions of GFP (pCLA108) and histone H3 (pCLA143) in combination with the orthogonal ribosome ribo-Q1 (pCLA96), depicted in Figure 5.2. In order to include the genes for the evolved MjAzFRS synthetases on one plasmid with the appropriate tRNA MjYT_UCCU, these were cloned into pDULE (pCLA144 to pCLA150).

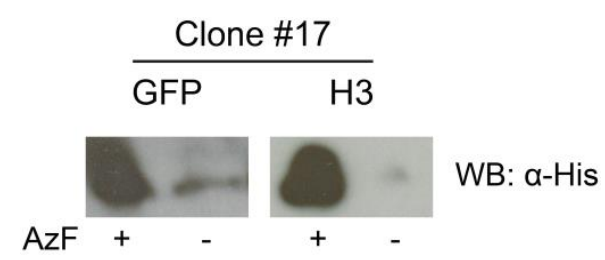

Figure 5.2: Incorporation of AzF into GFP and H3 using MjAzFRS library clone 17 (pCLA149).

Expressions of GFP 134AGGA (pCLA108) and H3 T6AGGA (pCLA143) were performed in the absence and presence of $1 \mathrm{mM}$ AzF. All orthogonal mRNA translations were driven by ribo-Q1 (pCLA96). For the western blot whole cell extracts were separated with SDS PAGE (Ch. 3.2.2.3) and blotted onto a PVDF membrane (Ch. 3.2.2.4). Anti-His-antibody was used as primary antibody. 
The MjAzFRS library clone 17 exhibited the best amino acid dependent expression and thereby AGGA codon suppressing efficiency. The gene for this mutant synthetase was finally cloned into the modular genetic tool ( $p C L A 155)$.

\subsection{Labeling of Proteins For FRET}

Förster/Fluorescence resonance energy transfer (FRET) is a powerful tool to investigate conformational changes of biomolecules like proteins and DNA, even on a single-molecule (sm) level ${ }^{[122,123]}$. Uemura et al. used FRET to study the transit of tRNAs on single translating ribosomes at codon resolution in real-time ${ }^{[176]}$. SMFRET experiments aided the understanding of the mechanism of Rrp44 (Dis3), a key catalytic subunit of the yeast exosome $^{[177]}$, and to obtain an experimentally derived model of the synaptotagmin 1-SNARE fusion complex ${ }^{[178]}$.

However, the precise and site-specific labeling of the proteins with suitable fluorophores is essential for FRET experiments but remains a challenging task and often the limiting factor. A combination of chemical ligations of fluorophores and proteins using maleimide conjugations and bioorthogonal labeling has been successfully used to study T4 lysozyme folding ${ }^{[124]}$. Nevertheless, this technique is restricted in its applicability to single-cysteine proteins. The incorporation of two distinct and suitable UAAs into the same protein ${ }^{[72,78]}$ and the subsequent bioorthogonal labeling of these provides an elegant method for the site-specifically installation of FRET pairs.

Genetically encoded UAAs with azide function are among those suitable, where they can be utilized as crosslinkers or be altered with bioorthogonal "click chemistry". Disadvantages of incorporating azides are that they suffer from intracellular reduction and, in the case of fluorescent labeling, the endangered design of a fluorogenic labeling scheme ${ }^{[119]}$. Alternative approaches are based on inverse electron-demand Diels-Alder cycloaddition reactions between genetically encoded strained alkenes and alkynes ${ }^{[119]}$, like norbornenes $^{[120]}$, bicyclononynes ${ }^{[121]}$ and trans-cyclooctenes ${ }^{[121]}$, and tetrazines and/or azides. Besides not having the disadvantages mentioned above, these kinds of bioorthogonal reactions have reported rate constants that are orders of magnitude faster than other established methods ${ }^{[179-182]}$. Furthermore, fluorophores conjugated to strongly quenching tetrazines can become intrinsically fluorogenic ${ }^{[119,183]}$, a trait difficult to attain if the tetrazines are genetically incorporated into proteins ${ }^{[119,184]}$, instead. 
The lysine derivatives NE-5-norbornene-2-yloxycarbonyl-L-lysine (NorK) and bicyclo[6.1.0]non-4-yn-9-ylmethanol-L-Iysine (BCNK) have been successfully incorporated into proteins by the Chin laboratory, followed by labeling with fluorophores conjugated to tetrazines ${ }^{[120,121]}$. Whereas the NorK could be incorporated using the wild type PylS/PylT pair, BCNK required a mutant PyIS (BCNRS) with the three amino acid substitutions Y271M, L274G, and C313A in the enzyme active site.

We performed an expression of an amber codon containing myoglobin (S4TAG; pCLA156) to test the incorporation of theses amino acids (Figure 5.3). The necessary mutations needed for PyIS to accept BCNK as a substrate were previously introduced by Heinz Neumann (pCLA158).

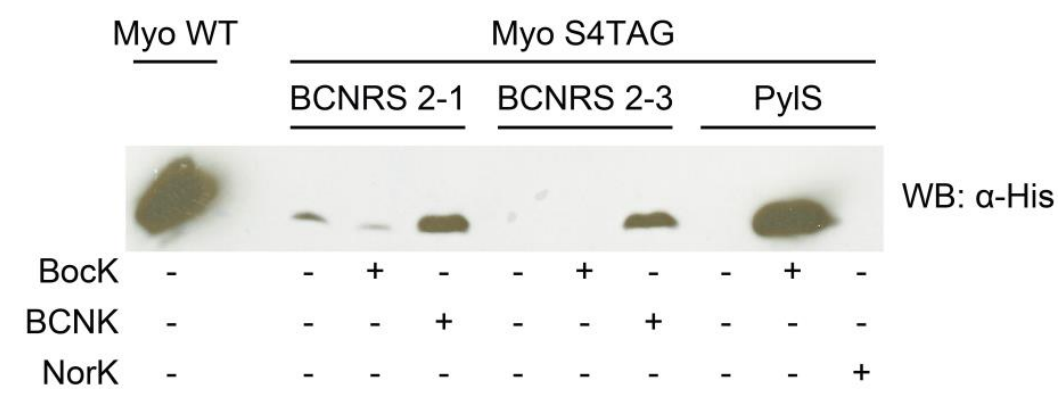

Figure 5.3: Incorporation of BCNK and NorK into myoglobin.

Expressions of wild type (Myo WT; pCLA157) and amber codon containing myoglobin (Myo S4TAG; pCLA156) were induced with arabinose (0.2\% final concentration) and tested in the absence of UAAs and presence of $1 \mathrm{mM}$ BocK, BCNK or Nork, respectively. Two different clones of the BCNK accepting PyIS (pCLA158) were tested. For the western blot whole cell extracts were separated with SDS PAGE (Ch. 3.2.2.3) and blotted onto a PVDF membrane (Ch. 3.2.2.4). Anti-His-antibody was used as primary antibody.

We observed specific incorporation of BCNK in response to the amber codon for both BCNRS synthetases. Although published, the wild type PylS was only tolerating the substrate analogue Bock but not the desired lysine derivative Nork.

To find another potential synthetase that is able to mediate the suppression of the amber codon with Nork without creating a new library, we tested a selection of existing PylS derivatives. These included the wild type PyIS (pCLA1), the BCNK employing BCNRS 2-1 (pCLA158), an evolved synthetase for photocaged lysine (pcKRS; pCLA159), an azidophotocaged lysine using variant (AzpcKRS; pCLA160) and two synthetases optimized for the incorporation of acetyllysine (AcK), namely AcKRS1 (pCLA161) and AcKRS3 (pCLA162). We chose an amber codon containing variant of histone H3 (K56TAG; pCLA163) as the target protein for the incorporation of Nork (Figure 5.4). 

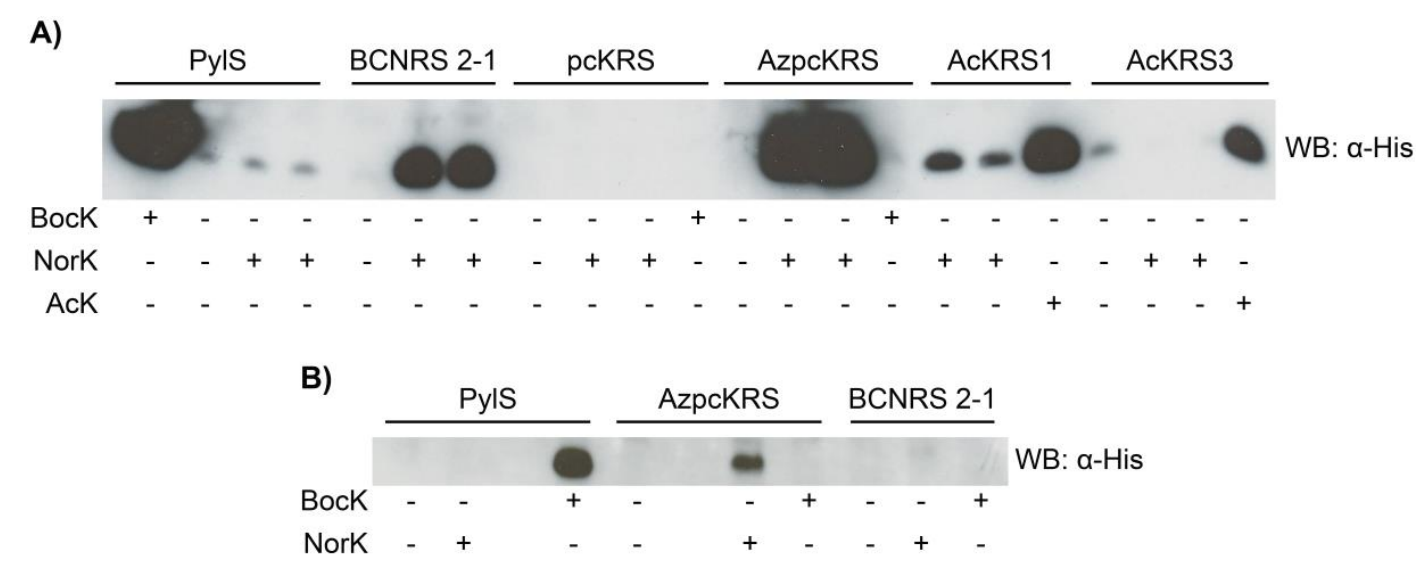

Figure 5.4: Incorporation of NorK into histone H3.

A) Expression of amber codon containing histone H3 (K56TAG; pCLA163) in E. coli BL21 was induced with IPTG (1 $\mathrm{mM}$ final concentration) and tested in the absence of UAAs and presence of $1 \mathrm{mM}$ BocK, NorK or AcK, respectively. Six different PylS derivatives were tested, including wild type (pCLA1), BCNRS 2-1 (pCLA158), pcKRS (pCLA159), AzpcKRS (pCLA160), AcKRS1 (pCLA161) and AcKRS3 (pCLA162). For the western blot whole cell extracts were separated with SDS PAGE (Ch. 3.2.2.3) and blotted onto a PVDF membrane (Ch. 3.2.2.4). Anti-Hisantibody was used as primary antibody. B) Same samples as in A) but five times less volume loaded onto gel.

The wild type PylS did not recognize Nork as a substrate, as already shown before (Figure 5.3). This means that no full-length histone could be detected (Figure 5.4-A). The synthetases for the photocaged lysine (pcKRS) as well as the acetyllysine (AcKRS3) were also not able to bind Nork. AcKRS1 showed some Nork dependent expression of $\mathrm{H} 3$ but clearly less than $\mathrm{H} 3$ with the actual corresponding amino acid AcK. BCNRS 2-1 seemed to incorporate Nork as efficient as the AcKRSs their substrate AcK. The azido-photocaged lysine aaRS revealed good yields of NorK dependent full-length histone H3. Expression levels were hard to compare with BocK dependent $\mathrm{H} 3$ expression using PylS due to overexposure on the western blot. A second blot with less sample loaded (Figure 5.4-B) verified that AzpcKRS is able to encode Nork with an efficiency approximately threefold lower than Bock encoded by wild type PyIS.

As a preparation for future FRET experiments we incorporated the amino acids BocK, BCNK and NorK into the single-cysteine protein histone H3 (V35TAG, F78C, C110A; pCLA164), in order to test the combination of tetrazine conjugated fluorophores with maleimide conjugations for in-gel pseudo FRET measurements. Therefore, the H3 histones were purified from E. coli inclusion bodies (Ch. 3.2.2.7) yielding $1.4 \mathrm{mg}$ protein with Bock, $1.1 \mathrm{mg}$ with Nork and $0.4 \mathrm{mg}$ with BCNK from $0.5 \mathrm{~L}$ culture each. Afterwards, the histones were consecutively labeled with fluorophores coupled to tetrazines and maleimides (Ch. 3.2.2.11). Labeling was verified and visualized using a Typhoon imager (Figure 5.5). 


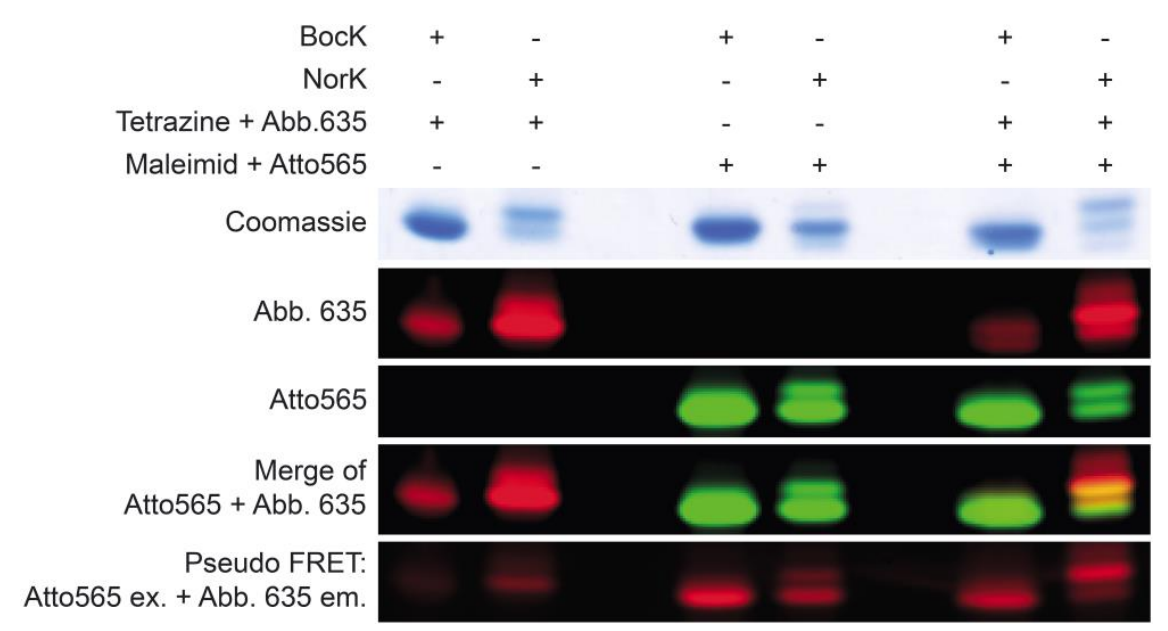

Figure 5.5: Labeling of histone H3 for (pseudo) FRET.

Nork and BocK were incorporated into histone H3 (V35TAG, F78C, C110A; PCLA164) with BocK serving as a negative control. Histone H3-NorK was labeled with the fluorophore Abberior Star635 (Abb. 635) conjugated with a tetrazine. BocK showed only weak interactions respective to protein level. For (pseudo) FRET an Atto565 dye conjugated with a maleimide was bound to the one cysteine. The SDS-PAGE gel was scanned using a Typhoon imager (Abb.635 - excitation (ex.): $633 \mathrm{~nm}$ (red laser) and emission (em.): Filter $670 \mathrm{~nm}$ BP30 / Atto565 - excitation: $532 \mathrm{~nm}$ (green laser) and emission: Filter $580 \mathrm{~nm}$ BP30).

With respect to the amount of protein loaded onto the gel only little background was observed. The tetrazine conjugated fluorophore Abberior Star635 could be specifically "clicked" to the strained alkene containing protein. The labeling of the protein, especially the double-labeling, caused a small shift of 1-2 kDa in the gel. This fits with the sizes of the dyes that have a molecular mass of approximately $1 \mathrm{kDa}$ each. The most shifted band belonged to $\mathrm{H} 3$ with NorK incorporated and double labeled with both dyes and showed a clear signal for pseudo FRET. That means the emission of the excited Atto565 fluorophore provoked the excitation of Abberior Star635 whose emission was finally detected. We obtained comparable results for labeling experiments with histones comprising BCNK instead of Nork (data not shown).

Finally, three additional tetrazine conjugated fluorescent dyes were tested for labeling using the same protein purifications as above (Figure 5.6).

The Typhoon scan showed only weak background signals for all four dyes tested as demonstrated by histones with non-reactive Bock incorporated. The labeling efficiencies for histones with NorK and BCNK were similar with Abberior Star635 and KK114 giving the brightest signals. It seemed that the four dyes did not have the same concentration, although properly dissolved with regard to their indicated weight, leading to distinct labeling efficiencies and thereby signal intensities. 


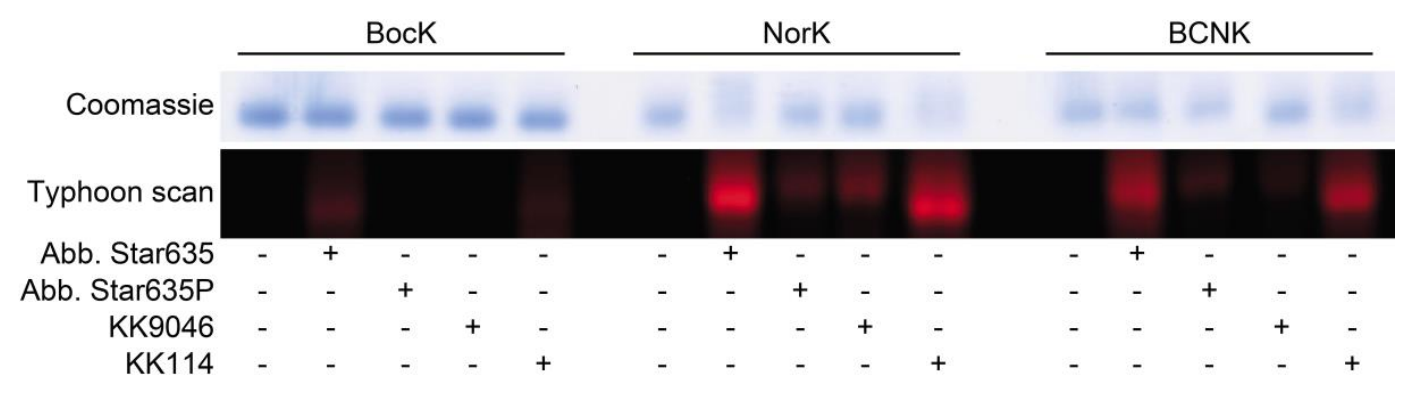

Figure 5.6: Labeling of histone $\mathrm{H3}$ with four different tetrazine conjugated fluorophores.

Nork, BCNK and BocK were incorporated into histone H3 (V35TAG, F78C, C110A; PCLA164) with BocK serving as a negative control. Histones were labeled with the tetrazine conjugated fluorophores Abberior (Abb.) Star635, Abberior Star635P, KK9046 and KK114 ${ }^{[125]}$. BocK showed only weak interactions respective to protein level. The SDS-PAGE gel was scanned using a Typhoon imager (Excitation: $633 \mathrm{~nm}$ (red laser) and emission: Filter $670 \mathrm{~nm}$ BP30).

In conclusion, the strained alkyne BCNK and alkene Nork could be genetically encoded into proteins that then could be site-specifically labeled with different fluorophores. We cloned the genes for both corresponding synthetases into the modular genetic tool substituting the wild type PyIS gene (pCLA165 and pCLA166). The UAA NorK can be combined with AzF, encoded by AGGA, whereas BCNK would cross-react with the azide. To potentially optimize the incorporation efficiency of both UAAs the wild type glnS promoter in front of both synthetases was replaced by the promising alternatives $P_{\text {con, }} P_{\text {lac }}$ (see Ch. 4.3 and 4.4.6; pCLA171 to pCLA174) and will ultimately be tested in future experiments.

\subsection{Fluorescently Labeled Nanobodies}

In 1993 Hamers and co-workers discovered antibodies in the camel serum that have naturally evolved to be devoid of light chains ${ }^{[185]}$. Later, these very small single-domain antibodies, about $15 \mathrm{kDa}^{[186]}$, were shown to be adaptable to other desired antigens by immunizing dromedaries followed by a screening process that led to extremely stable, highly soluble minimum sized antigen binders. These binders, termed nanobodies (Nb) by the Ablynx company, could be recombinantly expressed in $E$. coli and were proven to react specifically and with high affinity to the antigens ${ }^{[187]}$.

Due to their advantageous properties nanobodies have been used in a broad range of applications as research and diagnostic tools as well as therapeutics ${ }^{[188]}$. Saerens et al. found Nbs to sense conformational changes on different prostate-specific antigen isoforms which could be helpful to discriminate the stages of prostate cancer ${ }^{[189]}$. Nanobodies served as tools for molecular tumor imaging ${ }^{[190,191]}$ and were further developed for in vivo imaging of specific immune cell types ${ }^{[186]}$. The Leonhardt laboratory engineered an anti-GFP 
nanobody that allows the purification of GFP-fusion proteins and their interacting partners for biochemical studies on, e.g., DNA binding, enzymatic activity and complex formation ${ }^{[192]}$.

The Rizzoli group (ENI, Göttingen) is interested in synaptic vesicle function, with an emphasis on synaptic vesicle recycling, and therefore combines fluorescently labeled nanobodies and high resolution imaging provided by stimulated emission depletion (STED $^{[193,194]}$ ) microscopy. Chemical ligations of fluorophores and Nbs prevent a quantitative analysis due to multiple but erratic binding. Here, we show a collaborative approach for the site-specific incorporation of fluorophores, suitable for STED ${ }^{[125]}$, by applying the principles of genetic code expansion as described in Ch. 5.2.

First, a set of $\alpha$-synuclein targeting $\mathrm{Nbs}^{[195]}$ (NbSyn2), including wild type and the amber codon containing mutants S44TAG, K70TAG, R72TAG, K93TAG, K115TAG and S153TAG (provided by Felipe Opazo from AG Rizzoli),were expressed in the absence and presence of Bock and Nork to test the overall incorporation of UAAs into nanobodies (Figure 5.7).

A)

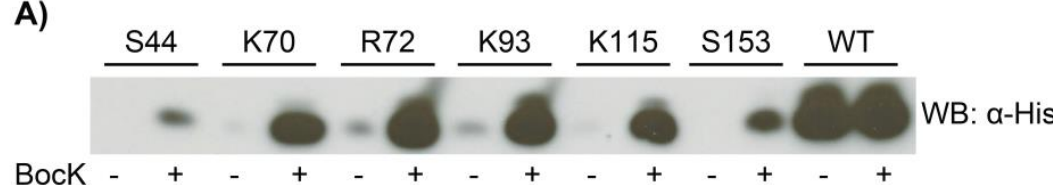

B)

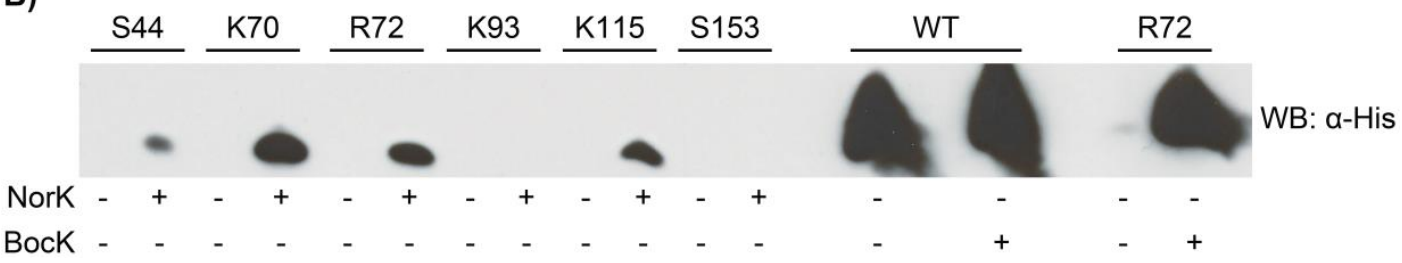

Figure 5.7: Incorporation of BocK and NorK into NbSyn2.

A) Expression of wild type (WT) and amber codon containing nanobodies NbSyn2 (pCLA175 to pCLA181) in E. coli BL21 was induced with IPTG (1 mM final concentration) after two hours of incubation at $37^{\circ} \mathrm{C}$. Bock (1 $\mathrm{mM}$ final concentration) was added subsequently and temperature was shifted to $28^{\circ} \mathrm{C}$ for overnight expression ( $\sim 16 \mathrm{~h}$ ). PylS and PylT were used as aaRS and tRNA pair (pCLA97). For the western blot whole cell extracts were separated with SDS PAGE (Ch. 3.2.2.3) and blotted onto a PVDF membrane (Ch. 3.2.2.4). Anti-Hisantibody was used as primary antibody. B) Same experiment as in A) but NorK was used as UAA instead of Bock together with the appropriate aaRS AzpcKRS (pCLA166). WT and R72 samples on the right side originate from A).

We found UAA dependent expression of NbSyn2 with BocK being incorporated with higher efficiencies than Nork yielding more full-length protein, as seen before (Figure 5.4-B). The clones with amber codons at positions $\mathrm{K} 70$ and R72 revealed the best expression levels. Both mutations are located in the same loop opposite the target binding pocket, according to the crystal structure of the nanobody ( $p d b$ file $2 X 6 \mathrm{M}^{[195]}$, not shown). 
We finally chose the NbSyn2 K70TAG clone for large scale expression and subsequent labeling with the Abberior Star635 fluorophore. Since the nanobody was cloned behind a secretion signal its production occurred in the periplasm, where the oxidizing environment is optimal for the required disulfide formation ${ }^{[188]}$. Secreted proteins were purified from the medium ( $1 \mathrm{~L}$; Ch. 3.2.2.8) yielding $0.11 \mathrm{mg}$ full-length protein and then labeled with the tetrazine conjugated fluorophore (Ch. 3.2.2.11). Labeling was verified and visualized using a Typhoon imager (Figure 5.8).

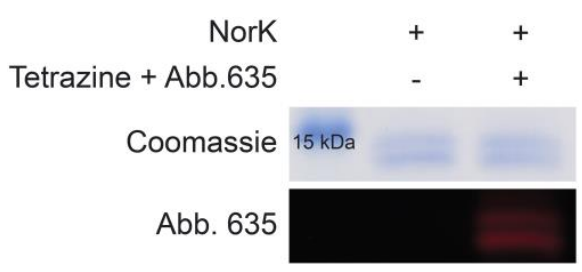

Figure 5.8: Fluorescently labeled NbSyn2.

NorK was incorporated into nanobody NbSyn2 (K70TAG; pCLA177). NbSyn2-NorK was labeled with the fluorophore Abberior Star635 (Abb. 635) conjugated with a tetrazine. The SDS-PAGE gel was scanned using a Typhoon imager (Excitation: $633 \mathrm{~nm}$ (red laser) and emission: Filter $670 \mathrm{~nm}$ BP30).

NbSyn2 could be successfully labeled with the tetrazine conjugated fluorophore Abberior Star635 and was given to Felipe Opazo for the removal of unbound dye and further experiments.

Due to the low yield of purified NbSyn2 from medium, we tested a second nanobody targeting GFP ${ }^{[192]}$ (NbGFP). NbGFP is expressed in good yields and exhibits excellent binding to its target. Additionally, the cell line was changed from E. coli BL21 to E. coli SHuffle. SHuffle cells were engineered to assist disulfide bond formation in the cytoplasm, e.g., in nanobodies, rendering the transport of proteins into the periplasm unnecessary (see NbSyn2). This is achieved by the simultaneous deletion of the reductases trxB and gor and the constitutive expression of a chromosomal copy of the disulfide bond isomerase DsbC which promotes the correction of mis-oxidized proteins into their correct form ${ }^{[196-198]}$. This cell line also possesses a disadvantage in that a chromosomal copy of the resistance gene for spectinomycin, which is also present on the pCDF plasmids used for the modular genetic tools.

To this end, we cloned the resistance genes for chloramphenicol and ampicillin from the Duet vectors PACYC and PET, respectively, into the modular genetic tools PCLA116 and pCLA171, replacing the spectinomycin resistance genes (pCLA184 to pCLA187). 
In order to test the genetic code expansion system in the SHuffle cell line, we prepared competent cells harboring the optimized modular genetic tool with an ampicillin resistance gene and encoding Bock in response to an amber codon (pCLA185). These cells were transformed with plasmids for NbGFP containing amber codons at positions Q13 and R76 (pCLA189 and pCLA192; provided by Felipe Opazo from AG Rizzoli). Nanobodies were expressed in the absence and presence of Bock (Figure 5.9).

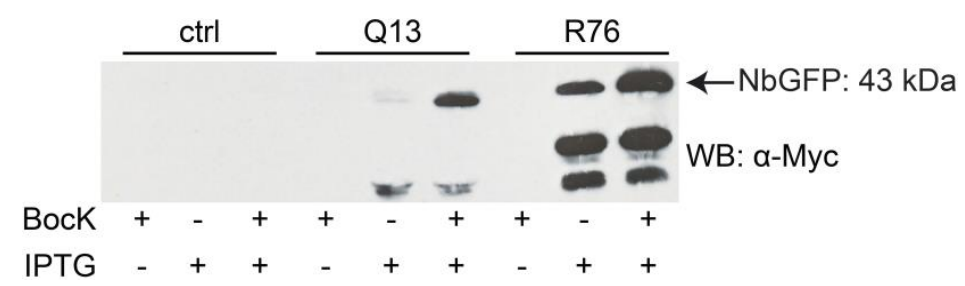

Figure 5.9: Incorporation of Bock into NbGFP.

Expression of amber codon containing nanobodies NbGFP (pCLA189 and pCLA192) in E. coli SHuffle was induced with IPTG (1 mM final concentration) after 2.5 hours of incubation at $30^{\circ} \mathrm{C}$. Bock ( $1 \mathrm{mM}$ final concentration) was added subsequently and temperature was shifted to $28^{\circ} \mathrm{C}$ for overnight expression $(\sim 16 \mathrm{~h})$. PylS and PylT were used as aaRS and tRNA pair (pCLA185). Control cells (ctrl) contained only pCLA185 but no NbGFP plasmid. For the western blot whole cell extracts were separated with SDS PAGE (Ch. 3.2.2.3) and blotted onto a PVDF membrane (Ch. 3.2.2.4). Anti-Myc-antibody was used as primary antibody.

The adapted modular genetic tool containing the ampicillin resistance gene allowed the incorporation of the UAA Bock into NbGFP. We found UAA dependent expression with clone R76TAG showing more full-length protein. Since the Myc-tag is cloned to the C-terminus of the protein only full-length nanobodies should be detected by the antibody. Therefore, the intense signals of smaller sizes are probably due to strong degradation.

In summary, we were able to encode UAAs in both nanobody proteins, NbSyn2 and NbGFP. NbSyn2 was successfully labeled with a fluorophore suitable for STED, opening an avenue for highly specific binding to its target followed by quantifiable high resolution microscopy. First experiments with NbGFP expression in E. coli SHuffle cells looked promising concerning high yield expression. The use of this cell line will simplify the purification of the nanobodies enormously.

\subsection{Orthogonal Ribosome Assisted In-Cell NMR Spectroscopy}

Living organisms are highly complex biological systems with intricate networks of biological processes, simultaneously exercised by an enormous number of macromolecules like proteins. The complexity is not only determined by the quantity of proteins expressed, but 
also by the combinatorial interactions between them. Deciphering these interactions and putting them into a meaningful context is required to fully understand how life works.

The in vivo investigation of proteins in their natural cellular environment within cells assures the perpetuation of structural conformations, which are of biological importance but not reproducible in vitro. Techniques like X-ray crystallography and high-resolution electron microscopy are usually confined to artificial, dilute, and isolated in vitro experimental setups because of their necessity for pure samples and crystalline or vitrified specimen ${ }^{[199]}$. Among several attempts to find new in vivo techniques for structural biology and cellular imaging ${ }^{[200]}$, in-cell nuclear magnetic resonance (NMR) technologies have been developed ${ }^{[201]}$ allowing the direct monitoring of conformational changes at the atomic level of proteins with "NMR-active" atomic nuclei. Since protein purification is unnecessary, in-cell NMR spectroscopy can be applied to studies of proteins that are difficult to purify. Proteolytically labile proteins would be advantageous for this method because they are better protected from the proteolytic machinery whose tight regulation in cells is lost in lysates ${ }^{[202]}$. Furthermore, in contrast to lysates, less protein amount is needed in intact cells due to higher local concentrations of interacting partners, thus increasing the likelihood of detecting weaker interactions ${ }^{[203]}$. Both qualitative structural ${ }^{[204]}$ and quantitative dynamic $^{[205,206]}$ data of proteins in living prokaryotic cells has been collected using high resolution NMR measurements.

The characterization of only one protein at a time was a limitation of the first developed in-cell NMR techniques ${ }^{[201]}$ because the simultaneous overexpression of more than one protein inside the cell led to extremely intricate NMR spectra. In order to study ubiquitin interactions, the Shekhtman laboratory used sequential protein expression in a timecontrolled manner to overcome this issue ${ }^{[203]}$. Here, we show an approach in collaboration with the Zweckstetter group (MPI BPC, Göttingen) that also regulates protein expression in a time-controlled fashion with the aid of an orthogonal ribosome evolved in the Chin laboratory ${ }^{[78]}$. The idea was to block protein synthesis by the endogenous ribosome using spectinomycin but to maintain translation of the desired protein by the mutant ribosome, which is spectinomycin resistant by virtue of a C1192U mutation in $16 \mathrm{~S} r \mathrm{RNA}^{[66]}$.

First trials of in-cell NMR experiments were performed in E. coli BL21 transformed with plasmids for the orthogonal ribosome ribo-Q1 (pCLA96) and an orthogonal mRNA coding for sfGFP ( $p C L A 105)$. Labeling experiments were grown in M9 minimal medium containing ${ }^{15} \mathrm{~N}$ labeled ammonium chloride for isotopic labeling of GFP (Ch. 3.2.2.10). ${ }^{15} \mathrm{~N}$ labeling schemes have been shown to exhibit negligible background levels ${ }^{[207]}$ compared to other 
labeling schemes using, e.g., ${ }^{13} \mathrm{C}$-isotopes. Purified ${ }^{15} \mathrm{~N}$ labeled GFP (1.534 mg protein out of $250 \mathrm{~mL}$ culture; Ch 3.2.2.9) and cells grown without the GFP mRNA were used as references. Despite a clear difference in GFP expression levels detected by western blot (Figure 5.10), in-cell NMR measurements, carried out by Martin Schwalbe from AG Zweckstetter, did not result in distinguishable NMR spectra for the GFP control or the control cells.

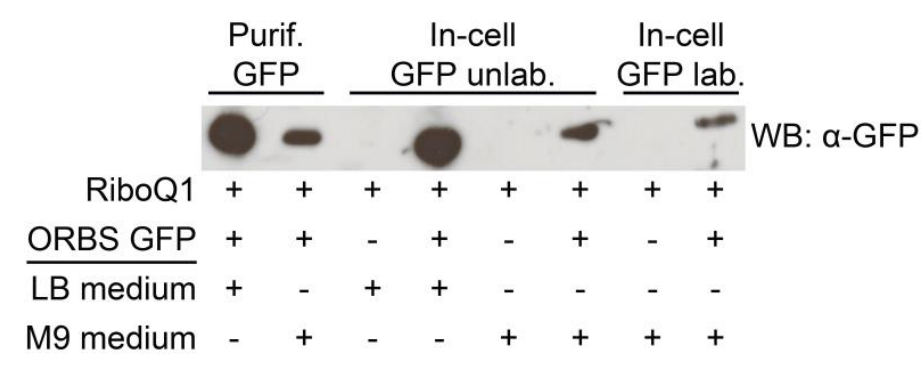

\section{Figure 5.10: sfGFP expression for in-cell NMR measurements.}

Expression of sfGFP in E. coli BL21 using an orthogonal mRNA (ORBS GFP; PCLA105) and the orthogonal ribosome ribo-Q1 (pCLA96). Samples were taken and normalized to $\mathrm{OD}_{600}=1.5$ from cells used for the purification of ${ }^{15} \mathrm{~N}$ labeled GFP (Purif. GFP; Ch 3.2.2.9) and for in-cell NMR measurements, each with samples from overnight cultures grown in LB medium and main cultures grown in $\mathrm{M9}$ minimal medium. Cells for in-cell $\mathrm{NMR}$ were grown in $\mathrm{M} 9$ medium with unlabeled $\mathrm{NH}_{4} \mathrm{Cl}$ (unlab.) to $\mathrm{OD}_{600}=0.6$ to 0.8 and subsequently transferred to $\mathrm{M} 9$ medium supplemented with ${ }^{15} \mathrm{~N}$ labeled (lab.) ${ }^{15} \mathrm{NH}_{4} \mathrm{Cl}$ and $150 \mu \mathrm{g} / \mathrm{mL}$ spectinomycin (Ch. 3.2.2.10) to block protein synthesis by the endogenous ribosome. For the western blot whole cell extracts were separated with SDS PAGE (Ch. 3.2.2.3) and blotted onto a PVDF membrane (Ch. 3.2.2.4). Anti-GFPantibody was used as primary antibody.

Although expression of GFP in M9 minimal medium yielded considerably less protein compared to expressions in LB medium, it was still sufficient to be clearly detected by western blot. However, using this method it was not possible to discriminate between the labeled and unlabeled form of GFP, with the latter being produced constitutively. To this end, we generated an arabinose inducible variant of the plasmid containing the orthogonal mRNA coding for sfGFP ( $\mathrm{PCLA193)}$ ) allowing the simultaneous blocking of the endogenous ribosome and the induction of orthogonal ribosome mediated ${ }^{15} \mathrm{~N}$ labeled GFP expression (Figure 5.11).

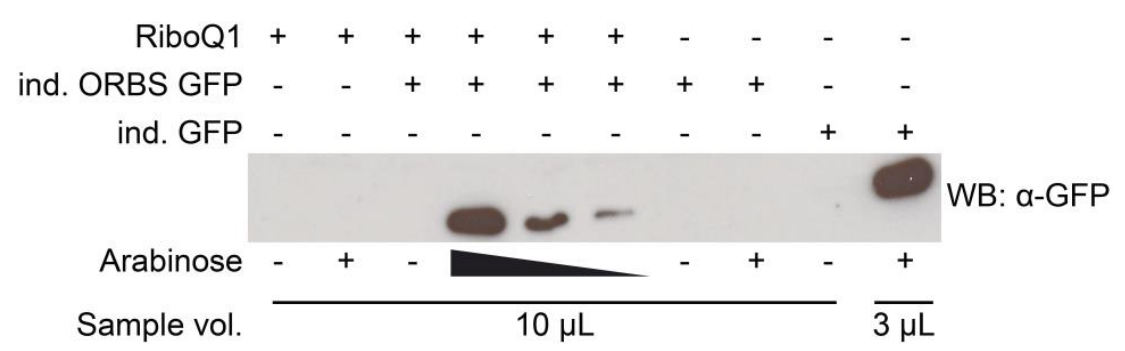

Figure 5.11: Arabinose inducible promoter for the orthogonal translational machinery.

Arabinose induced expression of sfGFP in E. coli BL21 using an orthogonal mRNA (ind. ORBS GFP; pCLA193) in combination with the orthogonal ribosome ribo-Q1 (pCLA96) and a non-orthogonal mRNA (ind. GFP; pCLA101). Black triangle indicates decreasing concentrations of arabinose $(0.2 \%, 0.04 \%$ and $0.008 \%)$. For the western blot whole cell extracts were separated with SDS PAGE (Ch. 3.2.2.3) and blotted onto a PVDF membrane (Ch. 3.2.2.4). Anti-GFP-antibody was used as primary antibody. 
We observed GFP expression that was dependent on the arabinose concentration only in the presence of the orthogonal ribosome. The expression efficiency was approximately eightfold lower in comparison to the original non-orthogonal pBAD vector, as depicted in Figure 5.11 and as confirmed by in-cell fluorescence measurement (not shown). In contrast, compared to the non-inducible orthogonal GFP encoding plasmid (pCLA105) we found a 2.5-fold decrement only (data not shown).

Both the non-orthogonal and the orthogonal inducible variants were used to investigate the effect of spectinomycin on cells and ribosomes, respectively, since the applied concentration of $150 \mu \mathrm{g} / \mathrm{mL}$ was supposed to be too harsh in order to produce acceptable amounts of ${ }^{15} \mathrm{~N}$ labeled GFP. Therefore, in-cell fluorescence of sfGFP was monitored over time in the absence and presence of $150 \mu \mathrm{g} / \mathrm{mL}$ spectinomycin (Figure 8.5) and samples were periodically taken for western blot analysis (Figure 5.12).

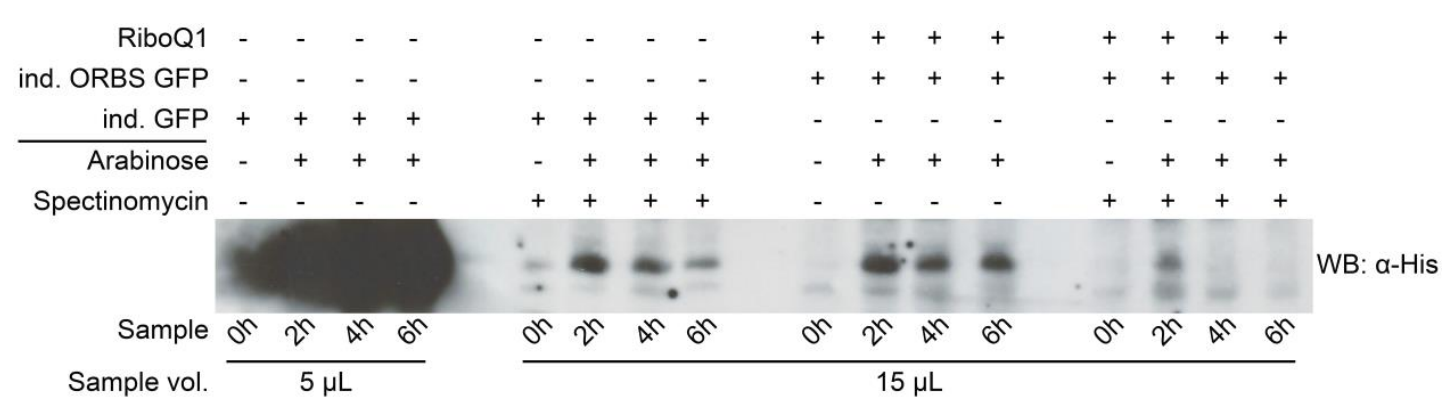

Figure 5.12: Effect of spectinomycin on sfGFP expression.

Arabinose induced expression of sfGFP in E. coli BL21 using an orthogonal mRNA (ind. ORBS GFP; pCLA193; $0.2 \%$ arabinose) in combination with the orthogonal ribosome ribo-Q1 (pCLA96) and a non-orthogonal mRNA (ind. GFP; pCLA101; 0.02\% arabinose). Expressions were performed in the absence and presence of $150 \mu \mathrm{g} / \mathrm{mL}$ spectinomycin. For the western blot whole cell extracts were separated with SDS PAGE (Ch. 3.2.2.3) and blotted onto a PVDF membrane (Ch. 3.2.2.4). Anti-His-antibody was used as primary antibody.

The in-cell fluorescence measurements (Figure 8.5) as well as the western blot (Figure 5.12) verified the expected effect of spectinomycin on non-orthogonal translational components with GFP levels being tremendously reduced. The combination of an inducible promoter and an orthogonal ribosomal binding site has been shown to produce less GFP than the non-orthogonal or non-inducible systems (Figure 5.11). However, it seems that the use of $150 \mu \mathrm{g} / \mathrm{mL}$ spectinomycin also had a strong negative influence on the orthogonal ribosome and thereby on the GFP expression efficiency.

In order to find a spectinomycin concentration that offers sufficient blocking of the endogenous ribosome while the orthogonal one is still mostly active, we repeated the in-cell fluorescence experiment with spectinomycin being titrated (Figure 8.6). A concentration of $10 \mu \mathrm{g} / \mathrm{mL}$ spectinomycin already decreased the final GFP fluorescence of 
cells with the non-orthogonal system by approximately $30 \%$. At $40 \mu \mathrm{g} / \mathrm{mL}$ spectinomycin only $35 \%$ of the fluorescence intensity from cells with unblocked ribosomes remained. Further increase of spectinomycin yielded only a moderate decrease in GFP levels to a maximum of approximately $17 \%$. Cells transformed with plasmids for the orthogonal components revealed that at $40 \mu \mathrm{g} / \mathrm{mL}$ spectinomycin there was still a fluorescence intensity of circa $56 \%$ compared to cells grown in medium without spectinomycin. Further supplements of spectinomycin led to a maximum loss of fluorescence of $60 \%$. Thus, a spectinomycin concentration of $40 \mu \mathrm{g} / \mathrm{mL}$ seemed to be sufficient for blocking endogenous ribosomes but keeping the orthogonal ones active enough. This was confirmed in a final experiment that analyzed GFP expression levels of cells grown in medium with the selected spectinomycin concentrations of 0,20 and $40 \mu \mathrm{g} / \mathrm{mL}$ by western blot (Figure 5.13). After normalizing cells according to in-cell fluorescence in the absence of spectinomycin (data not shown) only those containing the orthogonal mRNA coding for GFP and the orthogonal ribosome revealed GFP expression past treatment with $40 \mu \mathrm{g} / \mathrm{mL}$ spectinomycin.

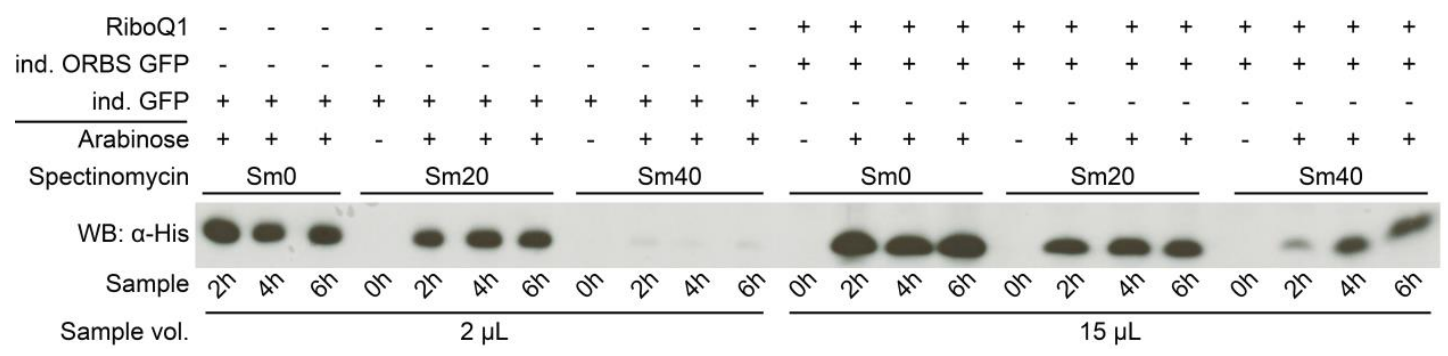

Figure 5.13: sfGFP expression with selected spectinomycin concentrations.

Arabinose induced expression of sfGFP in E. coli BL21 using an orthogonal mRNA (ind. ORBS GFP; pCLA193; $0.2 \%$ arabinose) in combination with the orthogonal ribosome ribo-Q1 (pCLA96) and a non-orthogonal mRNA (ind. GFP; pCLA101; $0.001 \%$ arabinose). Expressions were performed in the absence and presence of 20 or $40 \mu \mathrm{g} / \mathrm{mL}$ spectinomycin. Cells using orthogonal and non-orthogonal components were normalized by in-cell fluorescence of GFP (data not shown). For the western blot whole cell extracts were separated with SDS PAGE (Ch. 3.2.2.3) and blotted onto a PVDF membrane (Ch. 3.2.2.4). Anti-His-antibody was used as primary antibody.

The size of sfGFP, roughly $28 \mathrm{kDa}$, could present another reason that the previously described in-cell NMR experiment (Figure 5.10) did not work. The Zweckstetter group suggested testing the small peptide -NYHLENEVARLKKLVG-, representing the amino acids 16 to 31 of the yeast transcriptional activator GCN4, that has been used for NMR experiments before ${ }^{[208]}$. The peptide was expressed and ${ }^{15} \mathrm{~N}$ labeled as described for sfGFP but under the control of the arabinose inducible promoter (pCLA194) and with optimized conditions using only $40 \mathrm{\mu g} / \mathrm{mL}$ spectinomycin. However, we were again not able to detect the isotopically labeled peptide by NMR in living cells. 


\section{Discussion}

Studies on biological processes usually involve the examination of its participating proteins, which may be difficult, or impossible in some cases, using standard methods. In recent years, the expansion of the genetic code has become a powerful tool for biological studies. This technique provides the ability to incorporate unnatural amino acids, site-specifically into proteins, establishing new side chain possibilities and protein properties. The ongoing progress of this approach facilitated the expansion of the genetic code in several prokaryotic and eukaryotic organisms. However, the creation of proteins with (multiple) UAAs is significantly limited by the UAA incorporation efficiency. Efforts made to optimize this drawback are lacking in systematic studies that correlate aaRS and tRNA expression and aminoacylation levels with the efficiency of UAA incorporation ${ }^{[42,48,49,77]}$. This thesis focused on these issues by the establishment of assays to detect the abundance and activity of each component. We ultimately determined that the synthetase PyIS was responsible for restraining the performance of the system. Furthermore, we lowered the metabolic burden for the cell by balancing the expression levels of distinct components and by reducing the number of plasmids used. This enhanced cell viability and also provided a 5-10 fold increase in yields of proteins containing UAAs.

\subsection{Balancing An Expanded Genetic Code System}

One could suppose that the low efficiency of UAA incorporation could be easily adjusted by simply overexpressing the required components, i.e., the tRNA/aaRS pairs, and/or by increasing the UAA concentration in the media. However, the overexpression of endogenous components could also provoke toxic ancillary effects and exhaust biosynthetic energy. Moreover, UAAs, especially those that are not commercially obtainable, have to be used as economically as possible.

In order to determine optimal expression and aminoacylation levels of the tRNA/aaRS pairs, which guarantee sufficient suppression of the blank codons without distressing the cells, we developed assays for their detection. The absence of specific antibodies against the aaRS PyIS and MjYRS led us introduce the well-characterized small $\mathrm{His}_{6}$-tag into these proteins but neither the addition to the $\mathrm{N}$ - nor the $\mathrm{C}$-terminus allowed for clear detection of the synthetases by western blot using antibodies against the $\mathrm{His}_{6}$-tag. Since the cell lysates 
were treated under denaturing conditions for the blot, using SDS and heat, the accessibility of the terminal epitopes by antibodies should not be restricted. This was proven by the Schultz group which obtained a relatively weak but distinct signal of a C-terminal $\mathrm{His}_{6^{-}}$ tagged BPARS, a mutant MjYRS that decodes for BPA, in cell lysates by western blot analysis ${ }^{[69]}$. The detection of $\mathrm{His}_{6}$-tagged PylS also failed for them, until they used a variant that was codon-optimized for expression in $E$. coli ${ }^{[72]}$. Considering the crystal structures of both aaRS (Figure 4.2 and 4.10), their C-termini are exposed to the protein surfaces and in case of MjYRS not directed towards the tRNA. This holds also true for the N-terminus of MjYRS, but concerning PylS no statement can be made because structural data in combination with PylT and for the N-terminal domain is missing. However, unless there are unknown interactions with other components and as long as the proteins are properly folded, the $\mathrm{His}_{6}$-epitope should be accessible even under native conditions. Srinivasan et al. were therefore able to purify PylS using an N-terminal $\mathrm{His}_{6}-\mathrm{tag}^{[33]}$. An observation made by Chatterjee et al. ${ }^{[72]}$ and, independently by ourselves, is that PyIS is mostly found in the insoluble fraction, probably due to misfolding in E. coli. Improper folded proteins are prime targets for proteolysis ${ }^{[209]}$ and could thereby represent one explanation for the poor detectability of (tagged) PylS. We tried to solve this problem by using chaperones, reducing the temperature during expression and deleting parts of the proline-rich linker (Figure 4.1, 4.5 and 4.6) without success (data not shown).

In addition to these findings, we discovered that MjYRS accepted a $\mathrm{His}_{6}$-tag at the C-terminus without losing catalytic activity (Figure 4.11), whereas PyIS revealed a grave loss of activity upon addition of this tag (Figure 4.3). By contrast, shifting the $\mathrm{His}_{6}$-tag into the flexible linker region of PylS, between the N-terminus tRNA binding domain and the C-terminus catalytic domain, proved beneficial because we were then able to detect the non-codon-optimized protein by western blot without severely impairing its enzymatic functionality (Figure 4.3 and 4.4). The introduction of an unconventional internal $\mathrm{His}_{6}$-tag also helped us to determine MjYRS expression levels by western blot, which were many times lower than PyIS levels, although under the control of the same gInS promoter (Figure 4.12). When expressed from the same tacl promoter Chatterjee et al. observed MjYRS to be stronger expressed than $\mathrm{PyIS}^{[72]}$. This demonstrates how important different promoters can be for the regulation of certain protein levels and, in the case of these two aaRSs, very likely for the suppression efficiency of the blank codons.

When we quantified PyIS and MjYRS expression levels at different stages of growth in rich medium under the control of a glnS promoter, we found both proteins exhibited 
diminished expression levels when the cultures reached high cell densities (Figure 4.4 and 4.12). This was consistent with previous reports stating that, in E. coli, the enzyme level of the naturally regulated glutaminyl-tRNA synthetase increases with increasing growth rate ${ }^{[210]}$, because cultures that approach saturation exhibit decreased growth rates. Thus, the aaRSs may become limiting in their ability to charge their cognate tRNAs with UAAs, and thereby for suppression of blank codons in recombinant proteins, during prolonged expressions. This, in turn, renders the wild type gInS promoter amendable for this purpose. Indeed, the Schultz laboratory achieved a fivefold improved suppression efficiency using a mutant $g / n S$ promoter $\left(g / n S^{\prime}\right)$ that led to a twofold increase in BPARS expression ${ }^{[69]}$. Additional improvements were made by further raising the expression levels of different MjYRS derivatives using a dual gInS'/araBAD promoter system or a single tacl-driven aaRS expression cassette ${ }^{[51,70,72]}$

We decided to use promoter libraries for optimization, which have been shown to provide a broad range of promoter activities ${ }^{[158-160,211]}$. We determined, that for the PyIS, there was a direct positive dependency upon promoter strength for enzyme level, tRNA aminoacylation and suppression efficiency (Figure 4.19). Whereas Jensen and Hammer directly measured the effect of their synthetic promoter library in Lactococcus lactis and E.coli by $\beta$-galactosidase activity, obtaining up to 400 -fold variation in promoter strength ${ }^{[160]}$, we performed indirect measurements and observed a fivefold variation, only. This was determined by the ability of the PyIS to suppress an amber codon in the genes of CAT or histone $\mathrm{H} 3$, with the most efficient promoter being two to threefold more efficient than the wild type glnS variant. In the case of the MjYRS the promoter libraries did not reveal a similar tendency (Figure 4.22), leading to the assumption that the PyIS constrains the performance of the current system for the incorporation of two distinct UAAs. The replacement of the wild type glnS promoter in front of the PylS gene with some welldefined standard promoters of different strength, including the most promising candidate used by the Schultz group, i.e., $\operatorname{tacl}^{[72]}$, permitted us to enhance the performance of the system once more (Figure 4.25). Furthermore, we could characterize the gInS promoter and also its strongest library variant, $3 \mathrm{E}$, as relatively weak. However, we discovered modest increases in PylS levels already being advantageous, yielding higher amounts of UAA containing proteins. Promoters with stronger activities, like tacl, did not prove more useful and likely consumed biosynthetic resources, particularly if more than one component is controlled by these regulatory elements. To this end, we performed subsequent experiments using the constitutive promoter $\mathrm{P}_{\text {con }}$ or the IPTG inducible $\mathrm{P}_{\text {lac }}$ to carry out PyIS expression 
In order to optimize the expression levels of the appropriate tRNAs, PyIT and MjYT_UCCU, the original Ipp promoters were also exchanged by promoter libraries. DIG labeled hybridization probes were created for the simultaneous detection of their abundance and loading status by northern blot analysis. We obtained specific signals for both tRNAs and were able to distinguish their aminoacylated form from their free form by means of a mobility shift in acid urea gels (Figure 4.9 and 4.16), as demonstrated by Polycarpo et al. ${ }^{[39,212]}$ or Köhrer and Rajbhandary ${ }^{[142]}$. However, the effect of the tRNA promoter libraries on the suppression efficiency has not yet been investigated by western and northern blot. They were merely used in combination with the two aaRS promoter libraries to construct a four component suppressor plasmid (see Ch. 6.2). According to the results of the Schultz laboratory, where they replaced the weak Ipp promoter of MjYT_CUA by the stronger proK promoter $^{[69]}$, one could expect improved suppression efficiencies by increasing the tRNA expression levels. While they initially observed a positive correlation between suppression efficiency and increased copies of tRNA, up to six tRNA copies per plasmid organized in two clusters under the control of proK, they later stated that these increases caused toxicity effects to rise to significant levels, leading to considerably slowed growth rates of E. coli. They could show that a single copy of an optimized $M$. jannaschii amber suppressor tyrosyltRNA $^{[71]}$ exceeded the performance of multiple non-optimized tRNAs, while the cell growth was less affected ${ }^{[51]}$.

In summary, it was shown by us, and others, that raising expression levels of both aaRS and tRNA to a certain limit provides benefits to the genetic code expansion system. Expression levels beyond these limits are either producing toxic side effects or do not further enhance suppression efficiencies and are probably exhausting biosynthetic energy. Instead of increasing the component's abundance, additional opportunities to optimize the system could focus on the functionalities of the components. This is exemplified by the work of Kobayashi et al. who created a MjYRS mutant that aminoacylates its cognate tRNA 65 times more efficiently than its wild type variant, mainly due to improved recognition of the amber anticodon $^{[73]}$.

\subsection{Reducing The Metabolic Burden}

The efficient incorporation of two distinct unnatural amino acids, which can be altered by bioorthogonal "click chemistry", is an important intermediate step for the creation of proteins that can be double labeled with fluorophores for smFRET studies. Developed by the Chin laboratory, the original orthogonal ribosome assisted system for the dual 
suppression of an amber and a frameshift codon consisted of six exogenous components (two cognate pairs of tRNA/aaRS, an O-mRNA and the O-ribosome) distributed on four different plasmids ${ }^{[78]}$. This number of plasmids, harboring potentially toxic components and corresponding antibiotics for their maintenance, impairs cell growth rates significantly (Figure 4.38) and thus limits the possibilities of this system. By merging a tRNA/aaRS pair from two vectors into a single one, the reduction of required plasmids has already been shown to be advantageous for the incorporation of a single UAA into proteins ${ }^{[68,69]}$. Chatterjee et al. demonstrated the feasibility of a dual nonsense (amber and ochre) suppressor plasmid that was used to efficiently install two UAAs into GFP. This system did not take benefits from an O-ribosome; hence, only five exogenous components were present, distributed on two plasmids. However, protein yields were not improved compared to a three plasmid system ${ }^{[72]}$.

In contrast, we intended to optimize the six component system introduced by Chin and co-workers to profit from the evolved O-ribosome ribo-Q1 that facilitates UAA incorporation in response to both amber and frameshift codons, with the latter one providing 256 new possibilities to encode UAAs. We therefore constructed a modular genetic tool that also works as a dual suppressor plasmid by expressing both required tRNA/aaRS pairs, in order to reduce the metabolic burden provoked by the four plasmids of the original system. Furthermore, this tool permits the exchange of individual parts (including tRNAs, aaRS and their promoters and terminators) by a series of unique restriction sites to adapt the system for new UAAs (Figure 4.26). Compared to the established four plasmid system, we indeed observed significantly enhanced growth rates (Figure 4.38) and improved yields of UAA containing proteins (Figure 4.31) by employing our three plasmid system.

Trials to optimize our first version of the tool by replacing the original $g / n S$ and Ipp promters of the aaRS and tRNAs with promoter libraries, to balance the expression levels of each individual component, failed so far. It seemed that the replacement generated a multitude of less active or even inactive clones, which were difficult to separate from more efficient clones using FACS (Figure 4.34). The observed higher GFP expression levels from putatively optimized clones were possibly due to the simultaneous measurement of more than one cell by virtue of the small size of E. coli cells. In some cases a strong overexpression of PylS was found, while only tiny amounts of UAA containing GFP were produced (Figure 4.35). This is consistent with the aforementioned hypothesis that overexpressing one component is likely to produce toxicity and consume biosynthetic energy. 
By contrast, when we exchanged the gInS promoter of the PylS on the modular genetic tool with $\mathrm{P}_{\text {con }}$ or $\mathrm{P}_{\mathrm{lac}}$, we observed enhanced amber suppression efficiency, on its own and in combination with a frameshift codon. $P_{\text {con }}$ was at least 1.5 to 2 -fold more efficient and $\mathrm{P}_{\text {lac }}$ 2.5 to 3 -fold compared to the standard gInS promoter (Figure 4.36). In comparison with the original four plasmid system, these second optimized versions of our tool provided a 5-10 fold increase in yields of proteins containing UAAs, while the improved growth rates, due to the reduced metabolic burden, were maintained (Figure 4.37 and 4.38).

\subsection{The Avenue To FRET Measurements}

For a start to demonstrate the feasibility that the existing system can be optimized, all previously described optimizations were performed with the wild type PyIS and MjYRS using the commercially obtainable but unremarkable UAAs BocK and tyrosine as a substrate. The site-specific labeling of proteins with fluorophores by bioorthogonal "click chemistry" requires the incorporation of suitable UAAs and in some cases the evolution of the aaRS' specificity towards them. Chin et al. evolved MjYRS to incorporate one of these suitable UAAs, the azide containing UAA $p$-azido-L-phenylalanine (AzF), in response to an amber codon ${ }^{[80]}$. Later on, Neumann et al. changed the decoding property of this mutant aaRS (MjAzFRS) from UAG to AGGA by randomizing the tRNA anticodon recognizing region to accept the expanded anticodon of the MjYT_UCCU tRNA ${ }^{[78,79]}$. Since the amber codon is exclusive for the M. barkeri PyIS/PyIT pair in our optimized dual suppressor system, we also needed to evolve MjAzFRS (provided by the Chin laboratory) to incorporate AzF in response to AGGA. Using the published procedure ${ }^{[78,79]}$, we successfully obtained a MjAzFRS mutant that suppressed AGGA codons in GFP and histone H3 with high specificity for AzF (Figure 5.2). Sequencing of this, and other clones, revealed the common amino acid substitutions Y230K, C231K and P232K, but divergent mutations at positions F261, H283 and D286. This observation was also made by Neumann et al. who suggested that amino acids 230, 231 and 232 grant affinity and specificity for the anticodon, and that 261, 283 and 286 may link the identity of the anticodon to the amino acid identity ${ }^{[78]}$.

Furthermore, we were able to incorporate the strained alkyne bicyclo[6.1.0]non-4-yn-9ylmethanol-L-lysine (BCNK; Figure 5.3) and alkene NE-5-norbornene-2-yloxycarbonyl-Llysine (Nork; Figure 5.4) into proteins in response to amber codons using evolved variants of PyIS. Whereas BCNK incorporation was successful by means of issued PyIS mutations ${ }^{[121]}$, we could not produce Nork containing proteins with the aid of wild type PylS as published $^{[120]}$, but by the use of an alternative PyIS mutant originally designed to recognize 
an azido-photocaged lysine as substrate. The poor substrate specificity of wild type PylS for Nork was also found by Plass et al., who incorporated this amino acid and other strained alkene and alkyne UAAs using a rationally designed PylS double mutant ${ }^{[119]}$.

However, both BCNK and NorK contain chemical handles that can react with tetrazines and/or azides, allowing the labeling of proteins with fluorophores conjugated to these functional groups even in living cells ${ }^{[119-121]}$. To test this, as a preparation for future FRET experiments, we successfully labeled histone H3 proteins with different tetrazine conjugated fluorophores (Figure 5.5 and 5.6), which have been reported to exhibit superior performance in STED microscopy ${ }^{[125]}$. We even double labeled H3 by using a second maleimide conjugated fluorophore, permitting us to perform in-gel (pseudo) FRET measurements (Figure 5.5). Moreover, in collaboration with the Rizzoli group (ENI, Göttingen) we successfully expressed, and site-specifically labeled, the $\alpha$-synuclein targeting nanobody NbSyn2 ${ }^{[195]}$ with Abberior Star635, which is one of the tetrazine conjugated fluorophores (Figure 5.8). In contrast to classical chemical ligations of fluorophores and nanobodies, which occur protein wide and erratically, this approach will facilitate quantitative analyses in their research on synaptic vesicle function by STED microscopy.

In summary, we demonstrated the incorporation of the UAAs AzF, BCNK and NorK into different proteins in response to the frameshift codon AGGA or the amber codon UAG, respectively. Thereby, functional groups for bioorthogonal conjugations were introduced into these proteins, allowing us to site-specifically label them with fluorophores.

Each of the corresponding aaRS was cloned into our optimized genetic tool substituting the wild type MjYRS and PyIS genes. In order to produce proteins with two distinct UAAs that can be double labeled with fluorophores for smFRET studies, one could combine Nork and AzF but BCNK would cross-react with the azide of AzF. Initial trials to express proteins containing NorK and AzF yielded only tiny amounts of full-length protein, barely detectable by western blot (data not shown). This could have been due to the poor incorporation efficiency of Nork, which therefore have to be optimized for future experiments.

\subsection{Applications And Side Projects: Nanobodies And In-Cell NMR}

Improving proteins yields is generally important and desirable for most studies, including the production of fluorescently labeled nanobodies and the orthogonal ribosome assisted 
in-cell NMR spectroscopy. Since both applications/side projects were not part of the main focus of this thesis their optimization will only be briefly discussed.

We demonstrated that our optimized genetic tool was conducive to increase expression levels of proteins containing UAAs. However, the purification of the Nork containing nanobody NbSyn2 resulted only in very small amounts of protein $(0.11 \mathrm{mg}$ full-length protein out of $1 \mathrm{~L}$ medium). This was probably due to the poor incorporation efficiency of Nork but also due to the intricate but necessary production of NbSyn2 in the cell's periplasm ${ }^{[188]}$ and its release to the medium from which it was purified. Besides enhancing protein yields by optimizing the incorporation of Nork or using a more efficiently incorporated UAA, one could simplify the production and purification of the nanobody. Our first approach using the specialized E. coli SHuffle cell line turned out to be useful for the high yield expression of proteins that contain disulfide bonds (Figure 5.9).

The production of uniquely ${ }^{15} \mathrm{~N}$ labeled proteins for in-cell NMR experiments was based on the spectinomycin resistance of the used orthogonal ribosome ${ }^{[66]}$. We found that the initially applied concentration of $150 \mu \mathrm{g} / \mathrm{mL} \mathrm{Sm}$ was even too harsh for the O-ribosome, preventing an efficient protein synthesis (Figure 5.12). The reduction to $40 \mu \mathrm{g} / \mathrm{mL} \mathrm{Sm}$ showed a sufficient blocking of the endogenous ribosomes while the orthogonal ones were still active (Figure 5.13). However, a detection of isotopically labeled polypeptides by NMR in living cells was not possible. Maybe the amount of ${ }^{15} \mathrm{~N}$ labeled protein was too low to be detected despite optimized conditions. Additional mutations in the O-ribosome may increase the resistance to spectinomycin and thereby improve protein yields. We observed that the expression of the protein from the modified $\mathrm{PBAD}$ vector containing an ORBS was approximately eightfold lower in comparison to the original pBAD vector (Figure 5.11). This could perhaps be overcome by the use of a stronger inducible promoter, for example the IPTG driven lac or tacl promoter, or by optimizing the expression level of the O-ribosome.

\subsection{Concluding Remarks And Outlook}

Since synthetic biologists initiated the expansion of the genetic code more than a decade ago, it has become a powerful tool for basic and applied scientific research. Numerous efforts have been made to expand the catalogue of useful UAAs in several organisms, including prokaryotes and eukaryotes. We expect future developments to further extend the existing system by evolving new tRNA/aaRS pairs for the incorporation of novel UAAs in response to additional blank codons even in higher eukaryotes. Optimizing the translational 
apparatus for a more efficient production of proteins with multiple different UAAs will possibly allow its reprogramming to create fully synthetic unnatural polymers.

In this study, we described a part of these optimizations by significantly improving the reliability and yield of UAA containing proteins produced with the aid of orthogonal ribosomes. Our analysis of the limiting factors revealed bottlenecks of the existing system and will be helpful for future studies. For instance, the integration of some components into the host's chromosome for a further reduction of the metabolic burden, if one can find a solution to compensate the loss in copy numbers compared to plasmids. Optimized expression levels of O-ribosomes will also prove beneficial for the performance of the system. One approach, which addressed both concerns, was recently published by the Chin laboratory. That work combined the orthogonal ribosomal RNA of ribo-Q1 and the O-mRNA of the protein of interest on a single vector, reducing the number of required plasmids by one $^{[213]}$. Furthermore, they evolved a series of quadruplet-decoding PylT variants that showed enhanced UAA specific decoding of their corresponding codons on ribo-Q1 in combination with PylS or some of its derivatives in comparison with quadruplet-decoding pairs derived from MjYT/MjYRS. Both strategies together led to improved incorporation efficiencies of two distinct UAAs into the same protein and thus to the efficient genetically encoded installation of FRET pairs ${ }^{[213]}$. Moreover, the combination of their recent work and the work presented in this study will most likely lead to an extremely more efficient genetic code expansion system. 


\section{References}

[1] Woese, C. R.; Kandler, O. \& Wheelis, M. L. "Towards a natural system of organisms: proposal for the domains Archaea, Bacteria, and Eucarya." Proc. Natl. Acad. Sci. U. S. A. 87, 4576-9 (1990).

[2] May, R. M. "How many species are there on Earth?" Science 241, 1441-9 (1988).

[3] Mora, C.; Tittensor, D. P.; Adl, S.; Simpson, A. G. B. \& Worm, B. "How many species are there on Earth and in the ocean?" PLoS Biol. 9, e1001127 (2011).

[4] Tsugita, A. \& Fraenkel-Conrat, H. "The amino acid composition and C-terminal sequence of a chemically evoked mutant of TMV." Proc. Natl. Acad. Sci. U. S. A. 46, 636-42 (1960).

[5] Crick, F. H.; Barnett, L.; Brenner, S. \& Watts-Tobin, R. J. "General nature of the genetic code for proteins." Nature 192, 1227-32 (1961).

[6] Nirenberg, M. W.; Matthaei, J. H. \& Jones, O. W. "An intermediate in the biosynthesis of polyphenylalanine directed by synthetic template RNA." Proc. Natl. Acad. Sci. U. S. A. 48, 104-9 (1962).

[7] Nirenberg, M. \& Leder, P. "RNA Codewords and Protein Synthesis: The Effect of Trinucleotides upon the Binding of sRNA to Ribosomes" Science (80-. ). 145, 13991407 (1964).

[8] Khorana, H. G. "Polynucleotide synthesis and the genetic code." Harvey Lect. 62, 79105 (1966).

[9] Khorana, H. G. et al. "Polynucleotide synthesis and the genetic code." Cold Spring Harb. Symp. Quant. Biol. 31, 39-49 (1966).

[10] Crick, F. H. C. "The origin of the genetic code" J. Mol. Biol. 38, 367-379 (1968).

[11] Garen, A. "Sense and nonsense in the genetic code. Three exceptional triplets can serve as both chain-terminating signals and amino acid codons." Science 160, 14959 (1968).

[12] Ramakrishnan, V. "Ribosome structure and the mechanism of translation." Cell 108, 557-72 (2002).

[13] Schmeing, T. M. \& Ramakrishnan, V. "What recent ribosome structures have revealed about the mechanism of translation." Nature 461, 1234-42 (2009).

[14] Kozak, M. "Initiation of translation in prokaryotes and eukaryotes." Gene 234, 187208 (1999).

[15] Laursen, B. S.; Sørensen, H. P.; Mortensen, K. K. \& Sperling-Petersen, H. U. "Initiation of protein synthesis in bacteria." Microbiol. Mol. Biol. Rev. 69, 101-23 (2005). 
[16] Simonetti, a et al. "A structural view of translation initiation in bacteria." Cell. Mol. Life Sci. 66, 423-36 (2009).

[17] Shine, J. \& Dalgarno, L. "The 3'-terminal sequence of Escherichia coli 16 S ribosomal RNA: complementarity to nonsense triplets and ribosome binding sites." Proc. Natl. Acad. Sci. U. S. A. 71, 1342-6 (1974).

[18] Steitz, J. A. \& Jakes, K. "How ribosomes select initiator regions in mRNA: base pair formation between the $3^{\prime}$ terminus of $16 \mathrm{~S}$ rRNA and the mRNA during initiation of protein synthesis in Escherichia coli." Proc. Natl. Acad. Sci. U. S. A. 72, 4734-8 (1975).

[19] Woese, C. R.; Olsen, G. J.; Ibba, M. \& Söll, D. "Aminoacyl-tRNA synthetases, the genetic code, and the evolutionary process." Microbiol. Mol. Biol. Rev. 64, 202-36 (2000).

[20] O'Donoghue, P. \& Luthey-Schulten, Z. "On the evolution of structure in aminoacyltRNA synthetases." Microbiol. Mol. Biol. Rev. 67, 550-73 (2003).

[21] LaRiviere, F. J.; Wolfson, A. D. \& Uhlenbeck, O. C. "Uniform binding of aminoacyltRNAs to elongation factor Tu by thermodynamic compensation." Science 294, 1658 (2001).

[22] Ogle, J. M.; Murphy, F. V; Tarry, M. J. \& Ramakrishnan, V. "Selection of tRNA by the ribosome requires a transition from an open to a closed form." Cell 111, 721-32 (2002).

[23] Ogle, J. M.; Carter, A. P. \& Ramakrishnan, V. "Insights into the decoding mechanism from recent ribosome structures." Trends Biochem. Sci. 28, 259-66 (2003).

[24] Rodnina, M. V; Gromadski, K. B.; Kothe, U. \& Wieden, H.-J. "Recognition and selection of tRNA in translation." FEBS Lett. 579, 938-42 (2005).

[25] Crick, F. H. "Codon--anticodon pairing: the wobble hypothesis." J. Mol. Biol. 19, 54855 (1966).

[26] Rodnina, M. V \& Wintermeyer, W. "The ribosome as a molecular machine: the mechanism of tRNA-mRNA movement in translocation." Biochem. Soc. Trans. 39, 658-62 (2011).

[27] Capecchi, M. R. "Polypeptide chain termination in vitro: isolation of a release factor." Proc. Natl. Acad. Sci. U. S. A. 58, 1144-51 (1967).

[28] Scolnick, E.; Tompkins, R.; Caskey, T. \& Nirenberg, M. "Release factors differing in specificity for terminator codons." Proc. Natl. Acad. Sci. U. S. A. 61, 768-74 (1968).

[29] Zinoni, F.; Birkmann, A.; Stadtman, T. C. \& Böck, A. "Nucleotide sequence and expression of the selenocysteine-containing polypeptide of formate dehydrogenase (formate-hydrogen-lyase-linked) from Escherichia coli." Proc. Natl. Acad. Sci. U. S. A. 83, 4650-4 (1986). 
[30] Chambers, I. et al. "The structure of the mouse glutathione peroxidase gene: the selenocysteine in the active site is encoded by the "termination" codon, TGA." EMBO J. 5, 1221-7 (1986).

[31] Böck, A. et al. "Selenocysteine: the 21st amino acid." Mol. Microbiol. 5, 515-20 (1991).

[32] Böck, A.; Forchhammer, K.; Heider, J. \& Baron, C. "Selenoprotein synthesis: an expansion of the genetic code." Trends Biochem. Sci. 16, 463-7 (1991).

[33] Srinivasan, G.; James, C. M. \& Krzycki, J. a. "Pyrrolysine encoded by UAG in Archaea: charging of a UAG-decoding specialized tRNA." Science 296, 1459-62 (2002).

[34] Hao, B. et al. "A new UAG-encoded residue in the structure of a methanogen methyltransferase." Science 296, 1462-6 (2002).

[35] Atkins, J. F. \& Gesteland, R. "Biochemistry. The 22nd amino acid." Science 296, 1409-10 (2002).

[36] Driscoll, D. M. \& Copeland, P. R. "Mechanism and regulation of selenoprotein synthesis." Annu. Rev. Nutr. 23, 17-40 (2003).

[37] Krzycki, J. A. "The direct genetic encoding of pyrrolysine." Curr. Opin. Microbiol. 8, 706-12 (2005).

[38] Blight, S. K. et al. "Direct charging of tRNA(CUA) with pyrrolysine in vitro and in vivo." Nature 431, 333-5 (2004).

[39] Polycarpo, C. et al. "An aminoacyl-tRNA synthetase that specifically activates pyrrolysine." Proc. Natl. Acad. Sci. U. S. A. 101, 12450-4 (2004).

[40] Ambrogelly, A.; Palioura, S. \& Söll, D. "Natural expansion of the genetic code." Nat. Chem. Biol. 3, 29-35 (2007).

[41] Lobanov, A. V; Kryukov, G. V; Hatfield, D. L. \& Gladyshev, V. N. "Is there a twenty third amino acid in the genetic code?" Trends Genet. 22, 357-60 (2006).

[42] Neumann, H. "Rewiring translation - Genetic code expansion and its applications." FEBS Lett. 586, 2057-64 (2012).

[43] Furter, R. "Expansion of the genetic code: site-directed p-fluoro-phenylalanine incorporation in Escherichia coli." Protein Sci. 7, 419-26 (1998).

[44] Cropp, T. A. \& Schultz, P. G. "An expanding genetic code." Trends Genet. 20, 625-30 (2004).

[45] Wang, L.; Brock, A.; Herberich, B. \& Schultz, P. G. "Expanding the genetic code of Escherichia coli." Science 292, 498-500 (2001).

[46] Hartley, R. W. "Barnase and barstar. Expression of its cloned inhibitor permits expression of a cloned ribonuclease." J. Mol. Biol. 202, 913-5 (1988). 
[47] Chin, J. W. et al. "An expanded eukaryotic genetic code." Science 301, 964-7 (2003).

[48] Liu, C. C. \& Schultz, P. G. "Adding new chemistries to the genetic code." Annu. Rev. Biochem. 79, 413-44 (2010).

[49] Davis, L. \& Chin, J. W. "Designer proteins: applications of genetic code expansion in cell biology." Nat. Rev. Mol. Cell Biol. 13, 168-82 (2012).

[50] Bossi, L. "Context effects: translation of UAG codon by suppressor tRNA is affected by the sequence following UAG in the message." J. Mol. Biol. 164, 73-87 (1983).

[51] Young, T. S.; Ahmad, I.; Yin, J. A. \& Schultz, P. G. "An enhanced system for unnatural amino acid mutagenesis in E. coli." J. Mol. Biol. 395, 361-74 (2010).

[52] Gerdes, S. Y. et al. "Experimental determination and system level analysis of essential genes in Escherichia coli MG1655." J. Bacteriol. 185, 5673-84 (2003).

[53] Yamazaki, Y.; Niki, H. \& Kato, J. "Profiling of Escherichia coli Chromosome database." Methods Mol. Biol. 416, 385-9 (2008).

[54] Huang, Y. et al. "A convenient method for genetic incorporation of multiple noncanonical amino acids into one protein in Escherichia coli." Mol. Biosyst. 6, 6836 (2010).

[55] Rydén, S. M. \& Isaksson, L. A. "A temperature-sensitive mutant of Escherichia coli that shows enhanced misreading of UAG/A and increased efficiency for some tRNA nonsense suppressors." Mol. Gen. Genet. 193, 38-45 (1984).

[56] Ito, K.; Uno, M. \& Nakamura, Y. "Single amino acid substitution in prokaryote polypeptide release factor 2 permits it to terminate translation at all three stop codons." Proc. Natl. Acad. Sci. U. S. A. 95, 8165-9 (1998).

[57] Johnson, D. B. F. et al. "RF1 knockout allows ribosomal incorporation of unnatural amino acids at multiple sites." Nat. Chem. Biol. 7, 779-86 (2011).

[58] Johnson, D. B. F. et al. "Release factor one is nonessential in Escherichia coli." ACS Chem. Biol. 7, 1337-44 (2012).

[59] Mukai, T. et al. "Codon reassignment in the Escherichia coli genetic code." Nucleic Acids Res. 38, 8188-95 (2010).

[60] Li, X.; Yokota, T.; Ito, K.; Nakamura, Y. \& Aiba, H. "Reduced action of polypeptide release factors induces mRNA cleavage and tmRNA tagging at stop codons in Escherichia coli." Mol. Microbiol. 63, 116-26 (2007).

[61] Moore, S. D. \& Sauer, R. T. "The tmRNA system for translational surveillance and ribosome rescue." Annu. Rev. Biochem. 76, 101-24 (2007).

[62] Ohtake, K. et al. "Efficient decoding of the UAG triplet as a full-fledged sense codon enhances the growth of a prfA-deficient strain of Escherichia coli." J. Bacteriol. 194, 2606-13 (2012). 
[63] Wang, H. H. et al. "Programming cells by multiplex genome engineering and accelerated evolution." Nature 460, 894-8 (2009).

[64] Isaacs, F. J. et al. "Precise manipulation of chromosomes in vivo enables genomewide codon replacement." Science 333, 348-53 (2011).

[65] Lajoie, M. J. et al. "Genomically recoded organisms expand biological functions." Science 342, 357-60 (2013).

[66] Rackham, O. \& Chin, J. W. "A network of orthogonal ribosome x mRNA pairs." Nat. Chem. Biol. 1, 159-66 (2005).

[67] Wang, K.; Neumann, H.; Peak-Chew, S. Y. \& Chin, J. W. "Evolved orthogonal ribosomes enhance the efficiency of synthetic genetic code expansion." Nat. Biotechnol. 25, 770-7 (2007).

[68] Farrell, I. S.; Toroney, R.; Hazen, J. L.; Mehl, R. A. \& Chin, J. W. "Photo-cross-linking interacting proteins with a genetically encoded benzophenone." Nat. Methods 2, 377-84 (2005).

[69] Ryu, Y. \& Schultz, P. G. "Efficient incorporation of unnatural amino acids into proteins in Escherichia coli." Nat. Methods 3, 263-5 (2006).

[70] Cellitti, S. E. et al. "In vivo incorporation of unnatural amino acids to probe structure, dynamics, and ligand binding in a large protein by nuclear magnetic resonance spectroscopy." J. Am. Chem. Soc. 130, 9268-81 (2008).

[71] Guo, J.; Melançon, C. E.; Lee, H. S.; Groff, D. \& Schultz, P. G. "Evolution of amber suppressor tRNAs for efficient bacterial production of proteins containing nonnatural amino acids." Angew. Chem. Int. Ed. Engl. 48, 9148-51 (2009).

[72] Chatterjee, A.; Sun, S. B.; Furman, J. L.; Xiao, H. \& Schultz, P. G. "A versatile platform for single- and multiple-unnatural amino acid mutagenesis in Escherichia coli." Biochemistry 52, 1828-37 (2013).

[73] Kobayashi, T. et al. "Structural basis for orthogonal tRNA specificities of tyrosyl-tRNA synthetases for genetic code expansion." Nat. Struct. Biol. 10, 425-32 (2003).

[74] Doi, Y.; Ohtsuki, T.; Shimizu, Y.; Ueda, T. \& Sisido, M. "Elongation factor Tu mutants expand amino acid tolerance of protein biosynthesis system." J. Am. Chem. Soc. 129, 14458-62 (2007).

[75] Wan, W. et al. "A facile system for genetic incorporation of two different noncanonical amino acids into one protein in Escherichia coli." Angew. Chem. Int. Ed. Engl. 49, 3211-4 (2010).

[76] Anderson, J. C. et al. "An expanded genetic code with a functional quadruplet codon." Proc. Natl. Acad. Sci. U. S. A. 101, 7566-71 (2004).

[77] Wang, K.; Schmied, W. H. \& Chin, J. W. "Reprogramming the genetic code: from triplet to quadruplet codes." Angew. Chem. Int. Ed. Engl. 51, 2288-97 (2012). 
[78] Neumann, H.; Wang, K.; Davis, L.; Garcia-Alai, M. \& Chin, J. W. "Encoding multiple unnatural amino acids via evolution of a quadruplet-decoding ribosome." Nature 464, 441-4 (2010).

[79] Neumann, H.; Slusarczyk, A. L. \& Chin, J. W. "De novo generation of mutually orthogonal aminoacyl-tRNA synthetase/tRNA pairs." J. Am. Chem. Soc. 132, 2142-4 (2010).

[80] Chin, J. W. et al. "Addition of p-azido-L-phenylalanine to the genetic code of Escherichia coli." J. Am. Chem. Soc. 124, 9026-7 (2002).

[81] Ai, H.; Shen, W.; Sagi, A.; Chen, P. R. \& Schultz, P. G. "Probing protein-protein interactions with a genetically encoded photo-crosslinking amino acid." Chembiochem 12, 1854-7 (2011).

[82] Chou, C.; Uprety, R.; Davis, L.; Chin, J. W. \& Deiters, A. "Genetically encoding an aliphatic diazirine for protein photocrosslinking" Chem. Sci. 2, 480 (2011).

[83] Chin, J. W.; Martin, A. B.; King, D. S.; Wang, L. \& Schultz, P. G. "Addition of a photocrosslinking amino acid to the genetic code of Escherichia coli." Proc. Natl. Acad. Sci. U. S. A. 99, 11020-4 (2002).

[84] Mori, H. \& Ito, K. "Different modes of SecY-SecA interactions revealed by sitedirected in vivo photo-cross-linking." Proc. Natl. Acad. Sci. U. S. A. 103, 16159-64 (2006).

[85] Das, S. \& Oliver, D. B. "Mapping of the SecA.SecY and SecA.SecG interfaces by sitedirected in vivo photocross-linking." J. Biol. Chem. 286, 12371-80 (2011).

[86] Wilkins, B. J. et al. "A Cascade of Histone Modifications Induces Chromatin Condensation in Mitosis" Science (80-. ). 343, 77-80 (2014).

[87] Tagami, S. et al. "Crystal structure of bacterial RNA polymerase bound with a transcription inhibitor protein." Nature 468, 978-82 (2010).

[88] Neumann, H.; Hazen, J. L.; Weinstein, J.; Mehl, R. a \& Chin, J. W. "Genetically encoding protein oxidative damage." J. Am. Chem. Soc. 130, 4028-33 (2008).

[89] Liu, C. C. \& Schultz, P. G. "Recombinant expression of selectively sulfated proteins in Escherichia coli." Nat. Biotechnol. 24, 1436-40 (2006).

[90] Xie, J.; Supekova, L. \& Schultz, P. G. "A genetically encoded metabolically stable analogue of phosphotyrosine in Escherichia coli." ACS Chem. Biol. 2, 474-8 (2007).

[91] Park, H.-S. et al. "Expanding the genetic code of Escherichia coli with phosphoserine." Science 333, 1151-4 (2011).

[92] Nguyen, D. P.; Garcia Alai, M. M.; Kapadnis, P. B.; Neumann, H. \& Chin, J. W. "Genetically encoding N(epsilon)-methyl-L-lysine in recombinant histones." J. Am. Chem. Soc. 131, 14194-5 (2009). 
[93] Ai, H.-W.; Lee, J. W. \& Schultz, P. G. "A method to site-specifically introduce methyllysine into proteins in E. coli." Chem. Commun. (Camb). 46, 5506-8 (2010).

[94] Nguyen, D. P.; Garcia Alai, M. M.; Virdee, S. \& Chin, J. W. "Genetically directing $\varepsilon-N$, $\mathrm{N}$-dimethyl-L-lysine in recombinant histones." Chem. Biol. 17, 1072-6 (2010).

[95] Neumann, H.; Peak-Chew, S. Y. \& Chin, J. W. "Genetically encoding N(epsilon)acetyllysine in recombinant proteins." Nat. Chem. Biol. 4, 232-4 (2008).

[96] Virdee, S. et al. "Traceless and site-specific ubiquitination of recombinant proteins." J. Am. Chem. Soc. 133, 10708-11 (2011).

[97] Neumann, H. et al. "A method for genetically installing site-specific acetylation in recombinant histones defines the effects of H3 K56 acetylation." Mol. Cell 36, 15363 (2009).

[98] Lammers, M.; Neumann, H.; Chin, J. W. \& James, L. C. "Acetylation regulates cyclophilin A catalysis, immunosuppression and HIV isomerization." Nat. Chem. Biol. 6, 331-7 (2010).

[99] Ye, S.; Huber, T.; Vogel, R. \& Sakmar, T. P. "FTIR analysis of GPCR activation using azido probes." Nat. Chem. Biol. 5, 397-9 (2009).

[100] Ye, S. et al. "Tracking G-protein-coupled receptor activation using genetically encoded infrared probes." Nature 464, 1386-9 (2010).

[101] Schultz, K. C. et al. "A genetically encoded infrared probe." J. Am. Chem. Soc. 128, 13984-5 (2006).

[102] Deiters, A.; Geierstanger, B. H. \& Schultz, P. G. "Site-specific in vivo labeling of proteins for NMR studies." Chembiochem 6, 55-8 (2005).

[103] Jackson, J. C.; Hammill, J. T. \& Mehl, R. a. "Site-specific incorporation of a (19)Famino acid into proteins as an NMR probe for characterizing protein structure and reactivity." J. Am. Chem. Soc. 129, 1160-6 (2007).

[104] Li, C. et al. "Protein (19)F NMR in Escherichia coli." J. Am. Chem. Soc. 132, 321-7 (2010).

[105] Liu, C. C.; Qi, L.; Yanofsky, C. \& Arkin, A. P. "Regulation of transcription by unnatural amino acids." Nat. Biotechnol. 29, 164-8 (2011).

[106] Deiters, A.; Cropp, T. A.; Summerer, D.; Mukherji, M. \& Schultz, P. G. "Site-specific PEGylation of proteins containing unnatural amino acids." Bioorg. Med. Chem. Lett. 14, 5743-5 (2004).

[107] Giepmans, B. N. G.; Adams, S. R.; Ellisman, M. H. \& Tsien, R. Y. "The fluorescent toolbox for assessing protein location and function." Science 312, 217-24 (2006).

[108] Tsien, R. Y. "The green fluorescent protein." Annu. Rev. Biochem. 67, 509-44 (1998). 
[109] Wang, L.; Brock, A. \& Schultz, P. G. "Adding L-3-(2-Naphthyl)alanine to the genetic code of E. coli." J. Am. Chem. Soc. 124, 1836-7 (2002).

[110] Summerer, D. et al. "A genetically encoded fluorescent amino acid." Proc. Natl. Acad. Sci. U. S. A. 103, 9785-9 (2006).

[111] Wang, J.; Xie, J. \& Schultz, P. G. "A genetically encoded fluorescent amino acid." J. Am. Chem. Soc. 128, 8738-9 (2006).

[112] Lee, H. S.; Guo, J.; Lemke, E. a; Dimla, R. D. \& Schultz, P. G. "Genetic incorporation of a small, environmentally sensitive, fluorescent probe into proteins in Saccharomyces cerevisiae." J. Am. Chem. Soc. 131, 12921-3 (2009).

[113] Charbon, G. et al. "Localization of GroEL determined by in vivo incorporation of a fluorescent amino acid." Bioorg. Med. Chem. Lett. 21, 6067-70 (2011).

[114] Baskin, J. M. \& Bertozzi, C. R. "Bioorthogonal Click Chemistry: Covalent Labeling in Living Systems" QSAR Comb. Sci. 26, 1211-1219 (2007).

[115] Kolb, H. C.; Finn, M. G. \& Sharpless, K. B. "Click Chemistry: Diverse Chemical Function from a Few Good Reactions." Angew. Chem. Int. Ed. Engl. 40, 2004-2021 (2001).

[116] Agard, N. J.; Prescher, J. A. \& Bertozzi, C. R. "A strain-promoted [3 + 2] azide-alkyne cycloaddition for covalent modification of biomolecules in living systems." J. Am. Chem. Soc. 126, 15046-7 (2004).

[117] Baskin, J. M. et al. "Copper-free click chemistry for dynamic in vivo imaging." Proc. Natl. Acad. Sci. U. S. A. 104, 16793-7 (2007).

[118] Chakraborty, A. et al. "Opening and closing of the bacterial RNA polymerase clamp." Science 337, 591-5 (2012).

[119] Plass, T. et al. "Amino acids for Diels-Alder reactions in living cells." Angew. Chem. Int. Ed. Engl. 51, 4166-70 (2012).

[120] Lang, K. et al. "Genetically encoded norbornene directs site-specific cellular protein labelling via a rapid bioorthogonal reaction." Nat. Chem. 4, 298-304 (2012).

[121] Lang, K. et al. "Genetic Encoding of bicyclononynes and trans-cyclooctenes for sitespecific protein labeling in vitro and in live mammalian cells via rapid fluorogenic Diels-Alder reactions." J. Am. Chem. Soc. 134, 10317-20 (2012).

[122] Weiss, S. "Measuring conformational dynamics of biomolecules by single molecule fluorescence spectroscopy." Nat. Struct. Biol. 7, 724-9 (2000).

[123] Selvin, P. R. "The renaissance of fluorescence resonance energy transfer." Nat. Struct. Biol. 7, 730-4 (2000).

[124] Brustad, E. M.; Lemke, E. A.; Schultz, P. G. \& Deniz, A. A. "A general and efficient method for the site-specific dual-labeling of proteins for single molecule fluorescence resonance energy transfer." J. Am. Chem. Soc. 130, 17664-5 (2008). 
[125] Wurm, C. A. et al. "Novel red fluorophores with superior performance in STED microscopy" Opt. Nanoscopy 1, 7 (2012).

[126] Dower, W. J.; Miller, J. F. \& Ragsdale, C. W. "High efficiency transformation of E. coli by high voltage electroporation." Nucleic Acids Res. 16, 6127-45 (1988).

[127] Laemmli, U. K. "Cleavage of structural proteins during the assembly of the head of bacteriophage T4." Nature 227, 680-5 (1970).

[128] Bradford, M. M. "A rapid and sensitive method for the quantitation of microgram quantities of protein utilizing the principle of protein-dye binding." Anal. Biochem. 72, 248-54 (1976).

[129] Julius, M. H.; Masuda, T. \& Herzenberg, L. A. "Demonstration that antigen-binding cells are precursors of antibody-producing cells after purification with a fluorescence-activated cell sorter." Proc. Natl. Acad. Sci. U. S. A. 69, 1934-8 (1972).

[130] Kessler, C. \& Manta, V. "Specificity of restriction endonucleases and DNA modification methyltransferases a review (Edition 3)." Gene 92, 1-248 (1990).

[131] Pingoud, A. \& Jeltsch, A. "Structure and function of type II restriction endonucleases." Nucleic Acids Res. 29, 3705-27 (2001).

[132] Mullis, K. B. \& Faloona, F. A. "Specific synthesis of DNA in vitro via a polymerasecatalyzed chain reaction." Methods Enzymol. 155, 335-50 (1987).

[133] Sharkey, D. J.; Scalice, E. R.; Christy, K. G.; Atwood, S. M. \& Daiss, J. L. "Antibodies as thermolabile switches: high temperature triggering for the polymerase chain reaction." Biotechnology. (N. Y). 12, 506-9 (1994).

[134] Ochman, H.; Gerber, A. S. \& Hartl, D. L. "Genetic applications of an inverse polymerase chain reaction." Genetics 120, 621-3 (1988).

[135] Williams, M.; Louw, A. I. \& Birkholtz, L.-M. "Deletion mutagenesis of large areas in Plasmodium falciparum genes: a comparative study." Malar. J. 6, 64 (2007).

[136] Don, R. H.; Cox, P. T.; Wainwright, B. J.; Baker, K. \& Mattick, J. S. "“Touchdown" PCR to circumvent spurious priming during gene amplification." Nucleic Acids Res. 19, 4008 (1991).

[137] Hecker, K. H. \& Roux, K. H. "High and low annealing temperatures increase both specificity and yield in touchdown and stepdown PCR." Biotechniques 20, 478-85 (1996).

[138] Sanger, F. \& Coulson, A. R. "A rapid method for determining sequences in DNA by primed synthesis with DNA polymerase." J. Mol. Biol. 94, 441-8 (1975).

[139] Sanger, F.; Nicklen, S. \& Coulson, A. R. "DNA sequencing with chain-terminating inhibitors." Proc. Natl. Acad. Sci. U. S. A. 74, 5463-7 (1977). 
[140] Chomczynski, P. \& Sacchi, N. "Single-step method of RNA isolation by acid guanidinium thiocyanate-phenol-chloroform extraction." Anal. Biochem. 162, 156-9 (1987).

[141] Chomczynski, P. "A reagent for the single-step simultaneous isolation of RNA, DNA and proteins from cell and tissue samples." Biotechniques 15, 532-4, 536-7 (1993).

[142] Köhrer, C. \& Rajbhandary, U. L. "The many applications of acid urea polyacrylamide gel electrophoresis to studies of tRNAs and aminoacyl-tRNA synthetases." Methods 44, 129-38 (2008).

[143] Pelta, J.; Livolant, F. \& Sikorav, J. L. "DNA aggregation induced by polyamines and cobalthexamine." J. Biol. Chem. 271, 5656-62 (1996).

[144] Limsuwun, K. \& Jones, P. G. "Spermidine acetyltransferase is required to prevent spermidine toxicity at low temperatures in Escherichia coli." J. Bacteriol. 182, 537380 (2000).

[145] Santoro, S. W.; Wang, L.; Herberich, B.; King, D. S. \& Schultz, P. G. "An efficient system for the evolution of aminoacyl-tRNA synthetase specificity." Nat. Biotechnol. 20, 1044-8 (2002).

[146] Wang, L. \& Schultz, P. G. "A general approach for the generation of orthogonal tRNAs." Chem. Biol. 8, 883-90 (2001).

[147] Tropberger, P. et al. "Regulation of transcription through acetylation of H3K122 on the lateral surface of the histone octamer." Cell 152, 859-72 (2013).

[148] Larkin, M. A. et al. "Clustal W and Clustal X version 2.0." Bioinformatics 23, 2947-8 (2007).

[149] Consortium, T. U. "Update on activities at the Universal Protein Resource (UniProt) in 2013." Nucleic Acids Res. 41, D43-7 (2013).

[150] Jiang, R. \& Krzycki, J. A. "PylSn and the homologous N-terminal domain of pyrrolysyltRNA synthetase bind the tRNA that is essential for the genetic encoding of pyrrolysine." J. Biol. Chem. 287, 32738-46 (2012).

[151] DeLano, W. L. "The PyMOL Molecular Graphics System, Version 1.1" Schrödinger LLC http://www.pymol.org (2002).

[152] Yanagisawa, T. et al. "Multistep engineering of pyrrolysyl-tRNA synthetase to genetically encode N(epsilon)-(o-azidobenzyloxycarbonyl) lysine for site-specific protein modification." Chem. Biol. 15, 1187-97 (2008).

[153] Berman, H. M. et al. "The Protein Data Bank." Nucleic Acids Res. 28, 235-42 (2000).

[154] Yanagisawa, T.; Ishii, R.; Fukunaga, R.; Nureki, O. \& Yokoyama, S. "Crystallization and preliminary X-ray crystallographic analysis of the catalytic domain of pyrrolysyl-tRNA synthetase from the methanogenic archaeon Methanosarcina mazei." Acta Crystallogr. Sect. F. Struct. Biol. Cryst. Commun. 62, 1031-3 (2006). 
[155] Herring, S. et al. "The amino-terminal domain of pyrrolysyl-tRNA synthetase is dispensable in vitro but required for in vivo activity." FEBS Lett. 581, 3197-203 (2007).

[156] Gruber, A. R.; Lorenz, R.; Bernhart, S. H.; Neuböck, R. \& Hofacker, I. L. "The Vienna RNA websuite." Nucleic Acids Res. 36, W70-4 (2008).

[157] Altschul, S. F. et al. "Gapped BLAST and PSI-BLAST: a new generation of protein database search programs." Nucleic Acids Res. 25, 3389-402 (1997).

[158] Alper, H.; Fischer, C.; Nevoigt, E. \& Stephanopoulos, G. "Tuning genetic control through promoter engineering." Proc. Natl. Acad. Sci. U. S. A. 102, 12678-83 (2005).

[159] Hammer, K.; Mijakovic, I. \& Jensen, P. R. "Synthetic promoter libraries--tuning of gene expression." Trends Biotechnol. 24, 53-5 (2006).

[160] Jensen, P. R. \& Hammer, K. "The sequence of spacers between the consensus sequences modulates the strength of prokaryotic promoters." Appl. Environ. Microbiol. 64, 82-7 (1998).

[161] Winship, P. R. "An improved method for directly sequencing PCR amplified material using dimethyl sulphoxide." Nucleic Acids Res. 17, 1266 (1989).

[162] Hung, T.; Mak, K. \& Fong, K. "A specificity enhancer for polymerase chain reaction." Nucleic Acids Res. 18, 4953 (1990).

[163] Berezhnoy, A. Y. \& Shckorbatov, Y. G. "Dependence of the E. coli promoter strength and physical parameters upon the nucleotide sequence." J. Zhejiang Univ. Sci. B 6, 1063-8 (2005).

[164] Deuschle, U.; Kammerer, W.; Gentz, R. \& Bujard, H. "Promoters of Escherichia coli: a hierarchy of in vivo strength indicates alternate structures." EMBO J. 5, 2987-94 (1986).

[165] Liu, M.; Tolstorukov, M.; Zhurkin, V.; Garges, S. \& Adhya, S. "A mutant spacer sequence between -35 and -10 elements makes the Plac promoter hyperactive and cAMP receptor protein-independent." Proc. NatI. Acad. Sci. U. S. A. 101, 6911-6 (2004).

[166] Knaus, R. \& Bujard, H. "PL of coliphage lambda: an alternative solution for an efficient promoter." EMBO J. 7, 2919-23 (1988).

[167] Pédelacq, J.-D.; Cabantous, S.; Tran, T.; Terwilliger, T. C. \& Waldo, G. S. "Engineering and characterization of a superfolder green fluorescent protein." Nat. Biotechnol. 24, 79-88 (2006).

[168] Stokes, A. L. et al. "Enhancing the utility of unnatural amino acid synthetases by manipulating broad substrate specificity." Mol. Biosyst. 5, 1032-8 (2009).

[169] Mukai, T. et al. "Adding I-lysine derivatives to the genetic code of mammalian cells with engineered pyrrolysyl-tRNA synthetases." Biochem. Biophys. Res. Commun. 371, 818-22 (2008). 
[170] Fleet, G. W. J.; Porter, R. R. \& Knowles, J. R. "Affinity Labelling of Antibodies with Aryl Nitrene as Reactive Group" Nature 224, 511-512 (1969).

[171] Escher, E.; Jost, R.; Zuber, H. \& Schwyzer, R. "p-Azido-L-Phenylalanine Peptides. I: Synthesis of Peptide Ligands for Chymotrypsin and Aminopeptidases." Isr. J. Chem. 12, 129-138 (1974).

[172] Krieg, U. C.; Walter, P. \& Johnson, A. E. "Photocrosslinking of the signal sequence of nascent preprolactin to the 54-kilodalton polypeptide of the signal recognition particle." Proc. Natl. Acad. Sci. U. S. A. 83, 8604-8 (1986).

[173] Chen, Y.; Ebright, Y. W. \& Ebright, R. H. "Identification of the target of a transcription activator protein by protein-protein photocrosslinking." Science 265, 90-2 (1994).

[174] Sánchez, M. A. L. "Generation of tRNA Synthetases Capable of Incorporating Azidophenylalanine For The Genetic Code Expansion of Escherichia coli." (2012).

[175] Motz, J. "Einbau von Azido-Phenylalanin und Acetyl- Lysin durch orthogonale evolvierte Translationskomponenten in Histon H3" (2012).

[176] Uemura, S. et al. "Real-time tRNA transit on single translating ribosomes at codon resolution." Nature 464, 1012-7 (2010).

[177] Lee, G.; Bratkowski, M. A.; Ding, F.; Ke, A. \& Ha, T. "Elastic coupling between RNA degradation and unwinding by an exoribonuclease." Science 336, 1726-9 (2012).

[178] Choi, U. B. et al. "Single-molecule FRET-derived model of the synaptotagmin 1SNARE fusion complex." Nat. Struct. Mol. Biol. 17, 318-24 (2010).

[179] Blackman, M. L.; Royzen, M. \& Fox, J. M. "Tetrazine ligation: fast bioconjugation based on inverse-electron-demand Diels-Alder reactivity." J. Am. Chem. Soc. 130, 13518-9 (2008).

[180] Devaraj, N. K.; Weissleder, R. \& Hilderbrand, S. a. "Tetrazine-based cycloadditions: application to pretargeted live cell imaging." Bioconjug. Chem. 19, 2297-9 (2008).

[181] Taylor, M. T.; Blackman, M. L.; Dmitrenko, O. \& Fox, J. M. "Design and synthesis of highly reactive dienophiles for the tetrazine-trans-cyclooctene ligation." J. Am. Chem. Soc. 133, 9646-9 (2011).

[182] Devaraj, N. K. \& Weissleder, R. "Biomedical applications of tetrazine cycloadditions." Acc. Chem. Res. 44, 816-27 (2011).

[183] Devaraj, N. K.; Hilderbrand, S.; Upadhyay, R.; Mazitschek, R. \& Weissleder, R. "Bioorthogonal turn-on probes for imaging small molecules inside living cells." Angew. Chem. Int. Ed. Engl. 49, 2869-72 (2010).

[184] Seitchik, J. L. et al. "Genetically encoded tetrazine amino acid directs rapid sitespecific in vivo bioorthogonal ligation with trans-cyclooctenes." J. Am. Chem. Soc. 134, 2898-901 (2012). 
[185] Hamers-Casterman, C. et al. "Naturally occurring antibodies devoid of light chains." Nature 363, 446-8 (1993).

[186] De Groeve, K. et al. "Nanobodies as tools for in vivo imaging of specific immune cell types." J. Nucl. Med. 51, 782-9 (2010).

[187] Arbabi Ghahroudi, M.; Desmyter, A.; Wyns, L.; Hamers, R. \& Muyldermans, S. "Selection and identification of single domain antibody fragments from camel heavychain antibodies." FEBS Lett. 414, 521-6 (1997).

[188] Muyldermans, S. "Nanobodies: natural single-domain antibodies." Annu. Rev. Biochem. 82, 775-97 (2013).

[189] Saerens, D. et al. "Single domain antibodies derived from dromedary lymph node and peripheral blood lymphocytes sensing conformational variants of prostatespecific antigen." J. Biol. Chem. 279, 51965-72 (2004).

[190] Cortez-Retamozo, V. et al. "Efficient cancer therapy with a nanobody-based conjugate." Cancer Res. 64, 2853-7 (2004).

[191] Huang, L. et al. "SPECT imaging with 99mTc-labeled EGFR-specific nanobody for in vivo monitoring of EGFR expression." Mol. Imaging Biol. 10, 167-75 (2008).

[192] Rothbauer, U. et al. "A versatile nanotrap for biochemical and functional studies with fluorescent fusion proteins." Mol. Cell. Proteomics 7, 282-9 (2008).

[193] Hell, S. W. \& Wichmann, J. "Breaking the diffraction resolution limit by stimulated emission: stimulated-emission-depletion fluorescence microscopy." Opt. Lett. 19, 780-2 (1994).

[194] Klar, T. A. \& Hell, S. W. "Subdiffraction resolution in far-field fluorescence microscopy." Opt. Lett. 24, 954-6 (1999).

[195] De Genst, E. J. et al. "Structure and properties of a complex of $\alpha$-synuclein and a single-domain camelid antibody." J. Mol. Biol. 402, 326-43 (2010).

[196] Bessette, P. H.; Aslund, F.; Beckwith, J. \& Georgiou, G. "Efficient folding of proteins with multiple disulfide bonds in the Escherichia coli cytoplasm." Proc. Natl. Acad. Sci. U. S. A. 96, 13703-8 (1999).

[197] Qiu, J.; Swartz, J. R. \& Georgiou, G. "Expression of active human tissue-type plasminogen activator in Escherichia coli." Appl. Environ. Microbiol. 64, 4891-6 (1998).

[198] Levy, R.; Weiss, R.; Chen, G.; Iverson, B. L. \& Georgiou, G. "Production of correctly folded Fab antibody fragment in the cytoplasm of Escherichia coli trxB gor mutants via the coexpression of molecular chaperones." Protein Expr. Purif. 23, 338-47 (2001).

[199] Selenko, P. \& Wagner, G. "NMR mapping of protein interactions in living cells." Nat. Methods 3, 80-1 (2006). 
[200] Sali, A.; Glaeser, R.; Earnest, T. \& Baumeister, W. "From words to literature in structural proteomics." Nature 422, 216-25 (2003).

[201] Serber, Z. \& Dötsch, V. "In-cell NMR spectroscopy." Biochemistry 40, 14317-23 (2001).

[202] Groll, M.; Bochtler, M.; Brandstetter, H.; Clausen, T. \& Huber, R. "Molecular machines for protein degradation." Chembiochem 6, 222-56 (2005).

[203] Burz, D. S.; Dutta, K.; Cowburn, D. \& Shekhtman, A. "Mapping structural interactions using in-cell NMR spectroscopy (STINT-NMR)." Nat. Methods 3, 91-3 (2006).

[204] Dedmon, M. M.; Patel, C. N.; Young, G. B. \& Pielak, G. J. "FlgM gains structure in living cells." Proc. Natl. Acad. Sci. U. S. A. 99, 12681-4 (2002).

[205] Bryant, J. E.; Lecomte, J. T. J.; Lee, A. L.; Young, G. B. \& Pielak, G. J. "Protein dynamics in living cells." Biochemistry 44, 9275-9 (2005).

[206] Li, C. et al. "Differential dynamical effects of macromolecular crowding on an intrinsically disordered protein and a globular protein: implications for in-cell NMR spectroscopy." J. Am. Chem. Soc. 130, 6310-1 (2008).

[207] Serber, Z.; Corsini, L.; Durst, F. \& Dötsch, V. "In-cell NMR spectroscopy." Methods Enzymol. 394, 17-41 (2005).

[208] Steinmetz, M. O. et al. "Molecular basis of coiled-coil formation." Proc. Natl. Acad. Sci. U. S. A. 104, 7062-7 (2007).

[209] Gottesman, S. "Proteases and their targets in Escherichia coli." Annu. Rev. Genet. 30, 465-506 (1996).

[210] Cheung, A. Y.; Watson, L. \& Söll, D. "Two control systems modulate the level of glutaminyl-tRNA synthetase in Escherichia coli." J. Bacteriol. 161, 212-8 (1985).

[211] Miksch, G.; Bettenworth, F.; Friehs, K. \& Flaschel, E. "The sequence upstream of the 10 consensus sequence modulates the strength and induction time of stationaryphase promoters in Escherichia coli." Appl. Microbiol. Biotechnol. 69, 312-20 (2005).

[212] Polycarpo, C. R. et al. "Pyrrolysine analogues as substrates for pyrrolysyl-tRNA synthetase." FEBS Lett. 580, 6695-700 (2006).

[213] Wang, K. et al. "Optimized orthogonal translation of unnatural amino acids enables spontaneous protein double-labelling and FRET." Nat. Chem. 6, 393-403 (2014). 


\section{Appendix}

\subsection{Plasmids}

Table 8.1: List of plasmids.

Details for the construction of plasmids made during this thesis (method, primers, template etc.) are listed in Table 8.3. Plasmids labeled with "Cambridge" were kindly provided by the Chin laboratory (MRC Laboratory of Molecular Biology, Cambridge). The remaining plasmids were made by other group members of AG Neumann as indicated.

\begin{tabular}{|c|c|c|c|}
\hline No. & Name & Resistance & Source \\
\hline pCLA1 & pBK PyIS & Kan & Cambridge \\
\hline pCLA2 & pBK MjYRS_TAG & Amp & Cambridge \\
\hline pCLA3 & pBK MjYRS_AGGA (2D12) & Amp & Cambridge \\
\hline pCLA4 & pREP PyIT & Tet & Cambridge \\
\hline pCLA5 & pREP YC JYCUA (MjYT_CUA) & Tet & Cambridge \\
\hline pCLA6 & pREP YC JYCUA AGGA2 (MjYT_UCCU) & Tet & Cambridge \\
\hline pCLA7 & pBK His ${ }_{6}$ PylS & Kan & Thesis \\
\hline pCLA8 & pBK H6C Pyls & Kan & Thesis \\
\hline pCLA9 & pBK H6 34AA lack to mazei (gap) PylS & Kan & Thesis \\
\hline pCLA10 & pBK H6 Loop206-216 in mazei PylS & Kan & Thesis \\
\hline pCLA11 & pBK H6 PylS linker delta 10 & Kan & Thesis \\
\hline pCLA12 & pBK H6 PylS linker delta 20 & Kan & Thesis \\
\hline pCLA13 & pBK H6 PylS linker delta 15 & Kan & Thesis \\
\hline pCLA14 & pBK H6 PylS linker delta 30 & Kan & Thesis \\
\hline pCLA15 & pBK H6 PylS linker delta 50 & Kan & Thesis \\
\hline pCLA16 & pBK H6 PylS linker delta C-terminus & Kan & Thesis \\
\hline pCLA17 & pBK H6 PylS linker delta N-terminus & Kan & Thesis \\
\hline pCLA18 & pBK $\mathrm{His}_{6}$ MjYRS_TAG & Amp & Thesis \\
\hline pCLA19 & pBK H6C MjYRS_TAG & Amp & Thesis \\
\hline pCLA20 & pBK H6 Loop25-29 MjYRS_TAG & Amp & Thesis \\
\hline pCLA21 & pDULE BPA (BPA aaRS + MjYT_CUA) & Tet & Cambridge \\
\hline pCLA22 & pBK H6 gap PylS_Prom Lib 1D & Kan & Thesis \\
\hline pCLA23 & pBK H6 gap PylS_Prom Lib 2E & Kan & Thesis \\
\hline pCLA24 & pBK H6 gap PylS_Prom Lib 3E & Kan & Thesis \\
\hline pCLA25 & pBK H6 gap PylS_Prom Lib 4F & Kan & Thesis \\
\hline pCLA26 & pBK H6 gap PylS_Prom Lib 6B & Kan & Thesis \\
\hline pCLA27 & pBK H6 gap PylS_Prom Lib 7A & Kan & Thesis \\
\hline pCLA28 & pBK H6 gap PylS_Prom Lib 8G & Kan & Thesis \\
\hline pCLA29 & pBK H6 gap PylS_Prom Lib 10D & Kan & Thesis \\
\hline pCLA30 & pBK H6 gap PylS_Prom Lib 11D & Kan & Thesis \\
\hline pCLA31 & pBK H6 gap PylS_Prom Lib 12H & Kan & Thesis \\
\hline pCLA32 & pCDFDuet-PylT H3 R52TAG & $\mathrm{Sm}$ & Liljan Hahn \\
\hline pCLA33 & pBK H6C MjYRS_AGGA_Prom Lib 1D & Amp & Thesis \\
\hline pCLA34 & pBK H6C MjYRS_AGGA_Prom Lib 2F & Amp & Thesis \\
\hline pCLA35 & pBK H6C MjYRS_AGGA_Prom Lib 3B & Amp & Thesis \\
\hline pCLA36 & pBK H6C MjYRS_AGGA_Prom Lib 4A & Amp & Thesis \\
\hline pCLA37 & pBK H6C MjYRS_AGGA_Prom Lib 6D & Amp & Thesis \\
\hline pCLA38 & pBK H6C MjYRS_AGGA_Prom Lib 7E & Amp & Thesis \\
\hline pCLA39 & pBK H6C MjYRS_AGGA_Prom Lib 8G & Amp & Thesis \\
\hline pCLA40 & pBK H6C MjYRS_AGGA_Prom Lib 9F & Amp & Thesis \\
\hline pCLA41 & pBK H6C MjYRS_AGGA_Prom Lib 10B & Amp & Thesis \\
\hline
\end{tabular}




\begin{tabular}{|c|c|c|c|}
\hline pCLA42 & pBK H6C MjYRS_AGGA_Prom Lib 11E & Amp & Thesis \\
\hline pCLA43 & pMyo4AGGA MjtRNAYuccu-3 & Tet & Cambridge \\
\hline pCLA44 & pREP PylT short_Prom Lib A4 & Tet & Thesis \\
\hline pCLA45 & pREP PylT short_Prom Lib C2 & Tet & Thesis \\
\hline pCLA46 & pREP PylT short_Prom Lib E7 & Tet & Thesis \\
\hline pCLA47 & pREP PylT short_Prom Lib F5 & Tet & Thesis \\
\hline pCLA48 & pREP PylT short_Prom Lib I3 & Tet & Thesis \\
\hline pCLA49 & pREP PylT short_Prom Lib 16 & Tet & Thesis \\
\hline pCLA50 & pREP PylT short_Prom Lib J7 & Tet & Thesis \\
\hline pCLA51 & pREP PylT short_Prom Lib K2 & Tet & Thesis \\
\hline pCLA52 & pREP PylT short_Prom Lib L5 & Tet & Thesis \\
\hline pCLA53 & pREP MjYT_UCCU short_Prom Lib B3 & Tet & Thesis \\
\hline pCLA54 & pREP MjYT_UCCU short_Prom Lib B8 & Tet & Thesis \\
\hline pCLA55 & pREP MjYT_UCCU short_Prom Lib D4 & Tet & Thesis \\
\hline pCLA56 & pREP MjYT_UCCU short_Prom Lib E1 & Tet & Thesis \\
\hline pCLA57 & pREP MjYT_UCCU short_Prom Lib G7 & Tet & Thesis \\
\hline pCLA58 & pREP MjYT_UCCU short_Prom Lib I2 & Tet & Thesis \\
\hline pCLA59 & pREP MjYT_UCCU short_Prom Lib I8 & Tet & Thesis \\
\hline pCLA60 & pREP MjYT_UCCU short_Prom Lib K6 & Tet & Thesis \\
\hline pCLA61 & pREP MjYT_UCCU short_Prom Lib L1 & Tet & Thesis \\
\hline pCLA62 & pBK H6 gap PylS_con Prom & Kan & Thesis \\
\hline pCLA63 & pBK H6 gap PylS_cp25 Prom & Kan & Thesis \\
\hline pCLA64 & pBK H6 gap PylS_lac1-6 Mt5 Prom & Kan & Thesis \\
\hline pCLA65 & pBK H6 gap PylS_lacP1 Prom & Kan & Thesis \\
\hline pCLA66 & pBK H6 gap PylS_lambda ConN25 Prom & Kan & Thesis \\
\hline pCLA67 & pBK H6 gap PylS_lambda Prom & Kan & Thesis \\
\hline pCLA68 & pBK H6 gap PylS_T5 D/E20 Prom & Kan & Thesis \\
\hline pCLA69 & pBK H6 gap PylS_T5 H207 Prom & Kan & Thesis \\
\hline pCLA70 & pBK H6 gap PylS_tacl Prom & Kan & Thesis \\
\hline pCLA71 & pBK H6C MjYRS_AGGA (2D12) & Amp & Thesis \\
\hline pCLA72 & pBK H6C MjYRS_AGGA_Sall-Term & Amp & Thesis \\
\hline pCLA73 & pBK H6 gap PylS_Ndel to Kpnl & Kan & Thesis \\
\hline pCLA74 & pBK H6 gap PylS-Kpnl_Pstl to Sacl & Kan & Thesis \\
\hline pCLA75 & pBK H6 gap PylS-KpnI-Sacl_Sall & Kan & Thesis \\
\hline pCLA76 & pBK H6 gap PylS-KpnI-Sacl-Sall_Notl & Kan & Thesis \\
\hline pCLA77 & pREP PylT short & Tet & Thesis \\
\hline pCLA78 & pREP PylT short_Notl & Tet & Thesis \\
\hline pCLA79 & pREP PylT short-Notl_Mfel & Tet & Thesis \\
\hline pCLA80 & pREP MjYT_UCCU short & Tet & Thesis \\
\hline pCLA81 & pREP MjYT_UCCU short_Mfel & Tet & Thesis \\
\hline pCLA82 & pREP MjYT_UCCU short-Mfel_Xhol & Tet & Thesis \\
\hline pCLA83 & pBK H6 gap PylS_Sall & Kan & Thesis \\
\hline pCLA84 & pBK H6 gap PylS_Notl & Kan & Thesis \\
\hline pCLA85 & pBK H6 gap PylS_Ndel-restore & Kan & Thesis \\
\hline pCLA86 & pBK H6 gap PylS_Ndel-restore_PM1 & Kan & Thesis \\
\hline pCLA87 & pBK H6 gap PylS_Ndel-restore_PM2 & Kan & Thesis \\
\hline pCLA88 & pBK H6 gap PylS_Ndel-restore_PM3 & Kan & Thesis \\
\hline pCLA89 & pBK H6 gap PylS_Ndel-restore_PM4 & Kan & Thesis \\
\hline pCLA90 & pBK H6 gap PylS_Ndel-restore_PM5 & Kan & Thesis \\
\hline pCLA91 & pCDF Duet-1 & $\mathrm{Sm}$ & Novagen \\
\hline pCLA92 & pCDF_H6C MjYRS_AGGA & $\mathrm{Sm}$ & Thesis \\
\hline
\end{tabular}




\begin{tabular}{|c|c|c|c|}
\hline pCLA93 & pCDF_H6C MjYRS_H6 gap PylS & Sm & Thesis \\
\hline pCLA94 & pCDF_H6C MjYRS_H6 gap PyIS_PyIT & Sm & Thesis \\
\hline pCLA95 & pCDF_H6C MjYRS_H6 gap PyIS_PyIT_MjYT_UCCU & $\mathrm{Sm}$ & Thesis \\
\hline pCLA96 & pSC101*-ribo-Q1 & Kan & Cambridge \\
\hline pCLA97 & pCDF PyIST & $\mathrm{Sm}$ & Cambridge \\
\hline pCLA98 & pDULE MjYRST (AGGA) & Tet & Cambridge \\
\hline pCLA99 & pO-gst-malE & Amp & Cambridge \\
\hline pCLA100 & pO-delta-gst-malE_MCS & Amp & Thesis \\
\hline pCLA101 & pBAD sfGFP WT & Amp & Cambridge \\
\hline pCLA102 & pBAD sfGFP 150TAG & Amp & Cambridge \\
\hline pCLA103 & pBAD sfGFP 134AGGA 150TAG-1 & Amp & Cambridge \\
\hline pCLA104 & pBAD sfGFP 134AGGA-1 & Amp & Cambridge \\
\hline pCLA105 & pO-sfGFP WT & Amp & Thesis \\
\hline pCLA106 & pO-sfGFP 150TAG & Amp & Thesis \\
\hline pCLA107 & pO-sfGFP 134AGGA 150TAG-1 & Amp & Thesis \\
\hline pCLA108 & pO-sfGFP 134AGGA-1 & Amp & Thesis \\
\hline pCLA109 & pCDF_MjYRS_PyIS_PyIT_MjYT_UCCU-mismatch & $\mathrm{Sm}$ & Thesis \\
\hline pCLA110 & pREP YC JYCUA delta tRNA & Tet & Cambridge \\
\hline pCLA111 & pO-CAT & Tet & Cambridge \\
\hline pCLA112 & pO-CAT T6TAG & Tet & Thesis \\
\hline pCLA113 & pO-CAT T6TAG D111AGGA & Tet & Thesis \\
\hline pCLA114 & pYOBB2 PylT-1 & $\mathrm{Cm}$ & Cambridge \\
\hline pCLA115 & pCDF_GTF_PylS_Prom Lib 3E & Sm & Thesis \\
\hline pCLA116 & pCDF_GTF_PyIS_con Prom & $\mathrm{Sm}$ & Thesis \\
\hline pCLA117 & pCDF_GTF_PyIS_lacP1 Prom & Sm & Thesis \\
\hline pCLA118 & G9GSTMBP Y17AGGA N234TAG-1 & Amp & Cambridge \\
\hline pCLA119 & pDULE AzFRS (TAG) & Tet & Cambridge \\
\hline pCLA120 & pBK AzFRS (TAG) & Kan & Thesis \\
\hline pCLA121 & pBK AzFRS_AGGA Lib1 & Kan & Thesis \\
\hline pCLA122 & pBK AzFRS_AGGA Lib2 & Kan & Thesis \\
\hline pCLA123 & pBK AzFRS_AGGA Lib3 & Kan & Thesis \\
\hline pCLA124 & pBK AzFRS_AGGA Lib4 & Kan & Thesis \\
\hline pCLA125 & pBK AzFRS_AGGA Lib5 & Kan & Thesis \\
\hline pCLA126 & pBK AzFRS_AGGA Lib6 & Kan & Thesis \\
\hline pCLA127 & pBK AzFRS_AGGA Lib7 & Kan & Thesis \\
\hline pCLA128 & pBK AzFRS_AGGA Lib8 & Kan & Thesis \\
\hline pCLA129 & pBK AzFRS_AGGA Lib9 & Kan & Thesis \\
\hline pCLA130 & pBK AzFRS_AGGA Lib10 & Kan & Thesis \\
\hline pCLA131 & pBK AzFRS_AGGA Lib11 & Kan & Thesis \\
\hline pCLA132 & pBK AzFRS_AGGA Lib12 & Kan & Thesis \\
\hline pCLA133 & pBK AzFRS_AGGA Lib13 & Kan & Thesis \\
\hline pCLA134 & pBK AzFRS_AGGA Lib14 & Kan & Thesis \\
\hline pCLA135 & pBK AzFRS_AGGA Lib15 & Kan & Thesis \\
\hline pCLA136 & pBK AzFRS_AGGA Lib16 & Kan & Thesis \\
\hline pCLA137 & pBK AzFRS_AGGA Lib17 & Kan & Thesis \\
\hline pCLA138 & pBK AzFRS_AGGA Lib18 & Kan & Thesis \\
\hline pCLA139 & pBK AzFRS_AGGA Lib19 & Kan & Thesis \\
\hline pCLA140 & pBK AzFRS_AGGA Lib20 & Kan & Thesis \\
\hline pCLA141 & pCDF Duet-PylT H3opt Q76C (pCHR029) & Sm & C. Hoffmann \\
\hline pCLA142 & pCDF Duet-PylT H3opt Q76C_T6AGGA & Sm & Thesis \\
\hline pCLA143 & pO-H3opt Q76C_T6AGGA & Amp & Thesis \\
\hline
\end{tabular}




\begin{tabular}{|c|c|c|c|}
\hline pCLA144 & pDULE AzFRS_AGGA Lib2 & Tet & Thesis \\
\hline pCLA145 & pDULE AzFRS_AGGA Lib3 & Tet & Thesis \\
\hline pCLA146 & pDULE AzFRS_AGGA Lib5 & Tet & Thesis \\
\hline pCLA147 & pDULE AzFRS_AGGA Lib6 & Tet & Thesis \\
\hline pCLA148 & pDULE AzFRS_AGGA Lib11 & Tet & Thesis \\
\hline pCLA149 & pDULE AzFRS_AGGA Lib17 & Tet & Thesis \\
\hline pCLA150 & pDULE AzFRS_AGGA Lib18 & Tet & Thesis \\
\hline pCLA151 & pBK AzFRS_BamHI_Sall (TAG) & Kan & K. Halder \\
\hline pCLA152 & pCDF_AzFRS_BamHI_Sall (TAG) & $\mathrm{Sm}$ & Thesis \\
\hline pCLA153 & pCDF_AzFRS_BamHI_Sall (TAG)_PyIT_MjYT_UCCU & $\mathrm{Sm}$ & Thesis \\
\hline pCLA154 & pCDF_PylT_MjYT_UCCU_AzFRS (AGGA) & Sm & Thesis \\
\hline pCLA155 & pCDF_PyIT_MjYT_UCCU_AzFRS (AGGA)_PylS & $\mathrm{Sm}$ & Thesis \\
\hline pCLA156 & pMyo4TAG PyIT & Tet & Cambridge \\
\hline pCLA157 & pMyo 4K PylT-1 (amber reverted to AAA (Lys)) & Tet & Cambridge \\
\hline pCLA158 & pBK BCNRS & Kan & H. Neumann \\
\hline pCLA159 & pBK pcKRS-1 & Amp & Cambridge \\
\hline pCLA160 & pBK AzpcK2-3 & Amp & Cambridge \\
\hline pCLA161 & pBK AcKRS1 & Kan & Cambridge \\
\hline pCLA162 & pBK AcKRS3 & Kan & Cambridge \\
\hline pCLA163 & pCDF PylT1 H3K56TAG-1 & Sm & Cambridge \\
\hline pCLA164 & pCDF Duet-PylT H3opt V35TAG F78C C110A & Sm & Liljan Hahn \\
\hline pCLA165 & pCDF_PyIT_MjYT_UCCU_AzFRS (AGGA)_BCNRS & $\mathrm{Sm}$ & Thesis \\
\hline pCLA166 & pCDF_PyIT_MjYT_UCCU_AzFRS (AGGA)_AzpcKRS & Sm & Thesis \\
\hline pCLA167 & pBK BCNRS_con Prom & Kan & Thesis \\
\hline pCLA168 & pBK BCNRS_lacP1 Prom & Kan & Thesis \\
\hline pCLA169 & pBK AzpcKRS_con Prom & Kan & Thesis \\
\hline pCLA170 & pBK AzpcKRS_lacP1 Prom & Kan & Thesis \\
\hline pCLA171 & pCDF_PylT_MjYT_AzFRS_BCNRS_con Prom & Sm & Thesis \\
\hline pCLA172 & pCDF_PyIT_MjYT_AzFRS_BCNRS_lacP1 Prom & $\mathrm{Sm}$ & Thesis \\
\hline pCLA173 & pCDF_PylT_MjYT_AzFRS_AzpcKRS_con Prom & Sm & Thesis \\
\hline pCLA174 & pCDF_PyIT_MjYT_AzFRS_AzpcKRS_lacP1 Prom & Sm & Thesis \\
\hline pCLA175 & pHEN6_NbSyn2_Thrombin_His 6 -tag & Amp & AG Rizzoli \\
\hline pCLA176 & 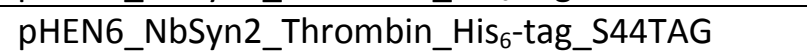 & Amp & AG Rizzoli \\
\hline pCLA177 & pHEN6_NbSyn2_Thrombin_His ${ }_{6}$-tag_K70TAG & Amp & AG Rizzoli \\
\hline pCLA178 & 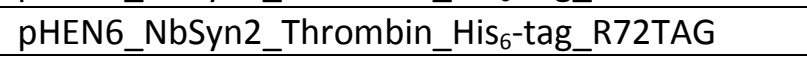 & Amp & AG Rizzoli \\
\hline pCLA179 & 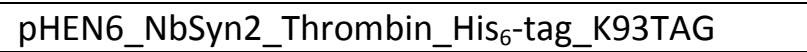 & Amp & AG Rizzoli \\
\hline pCLA180 & pHEN6_NbSyn2_Thrombin_His -tag_K115TAG $^{2}$ & Amp & AG Rizzoli \\
\hline pCLA181 & pHEN6_NbSyn2_Thrombin_His 6 -tag_K153TAG & Amp & AG Rizzoli \\
\hline pCLA182 & pACYC Duet & $\mathrm{Cm}$ & Novagen \\
\hline pCLA183 & pET Duet & Amp & Novagen \\
\hline pCLA184 & pCDF_GTF_PyIS_con Prom_CatR & $\mathrm{Cm}$ & Thesis \\
\hline pCLA185 & pCDF_GTF_PylS_con Prom_AmpR & Amp & Thesis \\
\hline pCLA186 & pCDF_PyIT_MjYT_AzFRS_BCNRS_con Prom_CatR & $\mathrm{Cm}$ & Thesis \\
\hline pCLA187 & pCDF_PyIT_MjYT_AzFRS_BCNRS_con Prom_AmpR & Amp & Thesis \\
\hline pCLA188 & K63_GFP_VHH_optimized_Nanobody & Kan & AG Rizzoli \\
\hline pCLA189 & K63_GFP_VHH_optimized_Nanobody_Q13TAG & Kan & AG Rizzoli \\
\hline pCLA190 & K63_GFP_VHH_optimized_Nanobody_S17TAG & Kan & AG Rizzoli \\
\hline pCLA191 & K63_GFP_VHH_optimized_Nanobody_R19TAG & Kan & AG Rizzoli \\
\hline pCLA192 & K63_GFP_VHH_optimized_Nanobody_R76TAG & Kan & AG Rizzoli \\
\hline pCLA193 & pBAD_ORBS_sfGFP WT & Amp & Thesis \\
\hline pCLA194 & pBAD_ORBS_GCN4p 16-31 & Amp & Thesis \\
\hline
\end{tabular}




\subsection{Oligonucleotides}

Oligonucleotides like primers and probes for RNA hybridization were ordered from Sigma-Aldrich (Steinheim) and dissolved with $\mathrm{ddH}_{2} \mathrm{O}$ as indicated on manufacturer's technical datasheet to a final concentration of $10 \mu \mathrm{M}$.

Table 8.2: List of oligos (primers and probes).

For $/ \mathrm{f}=$ forward; rev $/ \mathrm{r}=$ reverse.

\begin{tabular}{|c|c|c|}
\hline No. & Name & Sequence $5^{\prime} \rightarrow 3^{\prime}$ \\
\hline & H6Pylsf & $\begin{array}{l}\text { TGCATCACCATCACCACCATGATAAAAAACC } \\
\text { GCTG }\end{array}$ \\
\hline & H6PylSr & $\begin{array}{l}\text { TCATGGTGGTGATGGTGATGCATATGGGATT } \\
\text { CCTC }\end{array}$ \\
\hline $\mathrm{C3}$ & pBKseqf & CTCGGGTTGTCAGCCTGTC \\
\hline & $\mathrm{His}_{6} \mathrm{CPylSf}$ & $\begin{array}{l}\text { TGCACCATCACCATCATCACTAACTGCAGTT } \\
\text { TCAAAC }\end{array}$ \\
\hline & $\mathrm{His}_{6} \mathrm{CPylSr}$ & $\begin{array}{l}\text { TAGTGATGATGGTGATGGTGCAGGTTCGTGC } \\
\text { TAATG }\end{array}$ \\
\hline $\mathrm{C6}$ & pBKseqr & CAGAGATCATGTAGGCCTG \\
\hline $\mathrm{C7}$ & H6Lack34Pylsf & $\begin{array}{l}\text { GCGGGCACCATCACCATCATCACGGGAGCGT } \\
\text { TCCGGCGTC }\end{array}$ \\
\hline & H6Lack34PylSr & $\begin{array}{l}\text { CTCCCGTGATGATGGTGATGGTGCCCGCTGT } \\
\text { TCGGGGTG }\end{array}$ \\
\hline & H6LoopPyISf & $\begin{array}{l}\text { AAGGGCACCATCACCATCATCACGGGATGGC } \\
\text { GAAACCGTTTC }\end{array}$ \\
\hline & H6LoopPyISr & $\begin{array}{l}\text { ATCCCGTGATGATGGTGATGGTGCCCTTTAT } \\
\text { CTTCCGGAGAC }\end{array}$ \\
\hline & PylS_H6L_d10_f & $\begin{array}{l}\text { GAAAATAGCGTGAGCGTTCCGAGCCCGGCGA } \\
\text { AAAG }\end{array}$ \\
\hline & PylS_H6L_d10_r & $\begin{array}{l}\text { GGCTCGGAACGCTCACGCTATTTTCCAGCGG } \\
\text { TTTC }\end{array}$ \\
\hline & PylS_H6L_d20_f & $\begin{array}{l}\text { AAATAGCGTGAACAGCGGGCACCATCACCAT } \\
\text { CATC }\end{array}$ \\
\hline & PylS_H6L_d20_r & $\begin{array}{l}\text { GCCCGCTGTTCACGCTATTTTCCAGCGGTTT } \\
\text { CGGCGCAC }\end{array}$ \\
\hline & PylS_H6L_d15_f & $\begin{array}{l}\text { CACGGGAGCGTTCGTGTGGAAGCGCTGCTGT } \\
\text { CTCCGGAAG }\end{array}$ \\
\hline & PylS_H6L_d15_r & $\begin{array}{l}\text { CTTCCACACGAACGCTCCCGTGATGATGGTG } \\
\text { ATGGTG }\end{array}$ \\
\hline & PylS_H6L_d30_f & $\begin{array}{l}\text { CACGGGAGCGTTATGGCGAAACCGTTTCGTG } \\
\text { AACTG }\end{array}$ \\
\hline C18 & PylS_H6L_d30_r & $\begin{array}{l}\text { GTTTCGCCATAACGCTCCCGTGATGATGGTG } \\
\text { ATGGTG }\end{array}$ \\
\hline C19 & PylS_H6L_d50_f & $\begin{array}{l}\text { TGAACAGCGGGCACCATCACCATCATCACGG } \\
\text { GAGCGTTATGGCGAAACCGTTTCGTGAAC }\end{array}$ \\
\hline $\mathrm{C20}$ & PylS_H6L_d50_r & $\begin{array}{l}\text { ATAACGCTCCCGTGATGATGGTGATGGTGCC } \\
\text { CGCTGTTCACGCTATTTTCCAGCGGTTTC }\end{array}$ \\
\hline $\mathrm{C} 21$ & PylS_H6L_dCT_f & $\begin{array}{l}\text { CACGGGAGCGTTTAACTGCAGTTTCAAACGC } \\
\text { TAAATTG }\end{array}$ \\
\hline $\mathrm{C} 22$ & PylS_H6L_dCT_r & $\begin{array}{l}\text { ACTGCAGTTAAACGCTCCCGTGATGATGGTG } \\
\text { ATGGTG }\end{array}$ \\
\hline $\mathrm{C} 23$ & PylS_H6L_dNT_f & $\begin{array}{l}\text { ATCCCATATGAACAGCGGGCACCATCACCAT } \\
\text { CATC }\end{array}$ \\
\hline
\end{tabular}




\begin{tabular}{|c|c|c|}
\hline & PylS_H6L_dNT_r & $\begin{array}{l}\text { GTGCCCGCTGTTCATATGGGATTCCTCAAAG } \\
\text { CGTAAAC }\end{array}$ \\
\hline $\mathrm{C} 25$ & Mbarkeri_PylT_Probe & [CY3] GATTTAGAGTCCATTCGATC \\
\hline $\mathrm{C} 26$ & Mbarkeri_PylT_DIG_Probe & [DIG] GATTTAGAGTCCATTCGATC \\
\hline $\mathrm{C} 27$ & PylT_13-42 & ATGTAGATCGAATGGACTCTAAATCCGTTC \\
\hline $\mathrm{C} 28$ & T7P_PylT_for & $\begin{array}{l}\text { GAAATTAATACGACTCACTATAGGGAACCTG } \\
\text { ATCATGTAG }\end{array}$ \\
\hline $\mathrm{C} 29$ & T7P_PylT_rev & TGGCGGAAACCCCGGGAATCTAAC \\
\hline C30 & T7P_EcSerT_for & $\begin{array}{l}\text { GAAATTAATACGACTCACTATAGGGAGTGTG } \\
\text { GCCGAGCGGTTG }\end{array}$ \\
\hline C31 & T7P_EcSerT_rev & TGGCGGGAGCGCAGAGATTCGAAC \\
\hline C32 & Ecoli_serT_Probe & [CY3] GTTTTCAAGACCGGTGCCTT \\
\hline C33 & Ecoli_serT_DIG_Probe & [DIG] GTTTTCAAGACCGGTGCCTT \\
\hline C34 & H6JYRSf & $\begin{array}{l}\text { TGCATCACCATCACCACCATGACGAATTTGA } \\
\text { AATG }\end{array}$ \\
\hline C35 & H6JYRSr & $\begin{array}{l}\text { TCATGGTGGTGATGGTGATGCATATGGGATT } \\
\text { CCTC }\end{array}$ \\
\hline C36 & $\mathrm{His}_{6} \mathrm{cJYRSf}$ & $\begin{array}{l}\text { TACACCATCACCATCATCACTAACTGCAGTT } \\
\text { TCAAAC }\end{array}$ \\
\hline C37 & $\mathrm{His}_{6} \mathrm{CJYRSr}$ & $\begin{array}{l}\text { TAGTGATGATGGTGATGGTGTAATCTCTTTC } \\
\text { TAATTG }\end{array}$ \\
\hline C38 & H6LoopJYRSf & $\begin{array}{l}\text { ATGGGCACCATCACCATCATCACGGGGAAAA } \\
\text { ATCTGCTTACATAG }\end{array}$ \\
\hline C39 & H6LoopJYRSr & $\begin{array}{l}\text { TCCCCGTGATGATGGTGATGGTGCCCATCTT } \\
\text { TTTTTAAAACCTCTC }\end{array}$ \\
\hline $\mathrm{C} 40$ & MjYT_CUA_Probe & [CY5] GATTTAGAGTCCGCCGTTCT \\
\hline C41 & MjYT_CUA_DIG_Probe & [DIG] GATTTAGAGTCCGCCGTTCT \\
\hline C42 & T7P_MjTyrT_for & $\begin{array}{l}\text { GAAATTAATACGACTCACTATAGGGGCGGTA } \\
\text { GTTCAGCAGGGCAG }\end{array}$ \\
\hline $\mathrm{C} 43$ & T7P_MjTyrT_rev & TGGTGGGGCGGGCCGGATTTG \\
\hline C44 & MjYT_AGGA_DIG_Probe1 & [DIG] GGATTAGGAAGTCCGCCGTTCT \\
\hline $\mathrm{C} 45$ & MjYT_AGGA_DIG_Probe2 & [DIG] CAGCGCCATGCGGATTAGGAAG \\
\hline C46 & MjYT_AGGA_DIG_Probe3 & [DIG] GCCATGCGGATTAGGAAGTCCG \\
\hline C47 & MjYT_AGGA_DIG_Probe4 & [DIG] GCCATGCGGATTAGGA \\
\hline C48 & PylS_glnS_lib_f & $\begin{array}{l}\text { GCGCAGGAAAGGTCTCATATACGTTGTTTAC } \\
\text { GCTTTGAGGAATC }\end{array}$ \\
\hline & PylS_glnS_lib_r & $\begin{array}{l}\text { GCGCAGAGTAGGTCTCATATAACNNNNNATG } \\
\text { ATCNNNNNNNNNNNNNNNNNTGACAANNNNN } \\
\text { GTCGACGGATCCTTCAACTCAGCAAAAG }\end{array}$ \\
\hline $\mathrm{C50}$ & pBK_PylS_Sall_seq_f & GGTTGTAACACTGGCAGAG \\
\hline C51 & MJYRS_gInS_lib_r & $\begin{array}{l}\text { GCGCAGAGTAGGTCTCATATAACNNNNNATG } \\
\text { ATCNNNNNNNNNNNNNNNNNTGACAANNNNN } \\
\text { GAGGATCCTCTGACGCTCAGTGGAAC }\end{array}$ \\
\hline C52 & pBK_MjYRS-lib_seqf & TAAAAGGATCTAGGTGAAG \\
\hline C53 & pBK_Notl_Sall_seq_r & CTCGTCAGGGGGGCGGAGC \\
\hline & PylT_Ipp_lib_f & $\begin{array}{l}\text { GCGCAGGAAAGGTCTCAACGCTAGATCTGGG } \\
\text { AACCTGATCATG }\end{array}$ \\
\hline & PylT_Ipp_lib_r & $\begin{array}{l}\text { GCGCAGAGTAGGTCTCAGCGTTANNNNNATT } \\
\text { ACACAANNNNNNNNNNNNTGAGAANNNNNTT } \\
\text { TTGATGGGGCGCCACTTATTTTTG }\end{array}$ \\
\hline & MJYT_Ipp_lib_f & $\begin{array}{l}\text { GCGCAGGAAAGGTCTCAACGCTGAATTCCCG } \\
\text { GCGGTAGTTC }\end{array}$ \\
\hline C57 & pREP_tRNA_seq_r & GGGAGAGCGTCTGGCGAAAG \\
\hline
\end{tabular}




\begin{tabular}{|c|c|}
\hline C58 pBK_conProm_f & $\begin{array}{l}\text { GCGCAGGAAAGGTCTCACGGTTATAATGGTA } \\
\text { CCATAAGGAGGTGGATCCGGCAGTTTACGCT } \\
\text { TTGAGGAATC }\end{array}$ \\
\hline C59 pBK_conProm_r & $\begin{array}{l}\text { GCGCAGAGTAGGTCTCAACCGCCAAGCTTAA } \\
\text { AAATGTCAACAACGACGGTGAATTGTCGACG } \\
\text { GATCCTTCAACTC }\end{array}$ \\
\hline C60 $\quad$ pBK_CP25Prom_f & $\begin{array}{l}\text { GCGCAGGAAAGGTCTCAGAGGGGGCTGGTAT } \\
\text { AATCACATAGTACTGTTGTTTACGCTTTGAG } \\
\text { GAATC }\end{array}$ \\
\hline C61 pBK_CP25Prom_r & $\begin{array}{l}\text { GCGCAGAGTAGGTCTCACCTCACTACATGTC } \\
\text { AAGAATAAACTGCCAAAGGTCGACGGATCCT } \\
\text { TCAACTC }\end{array}$ \\
\hline C62 pBK_lac1-6Mt5Prom_f & $\begin{array}{l}\text { GCGCAGGAAAGGTCTCATGTTTATGTTGTGT } \\
\text { GGAATTGTGAGCGGATAACAATGTTTACGCT } \\
\text { TTGAGGAATC }\end{array}$ \\
\hline C63 pBK_lac1-6Mt5Prom_r & $\begin{array}{l}\text { GCGCAGAGTAGGTCTCAAACATAAATTGCTT } \\
\text { AAAGTGTAAAGCCTGGGGTGCCTAAGTCGAC } \\
\text { GGATCCTTCAACTC }\end{array}$ \\
\hline C64 pBK_lacP1Prom_f & $\begin{array}{l}\text { GCGCAGGAAAGGTCTCACTGGTATGTTGTGT } \\
\text { GGAATTGTGAGCGGATAACAATGTTTACGCT } \\
\text { TTGAGGAATC }\end{array}$ \\
\hline C65 pBK_lacP1Prom_r & $\begin{array}{l}\text { GCGCAGAGTAGGTCTCACCAGCCGGAAGCAT } \\
\text { AAAGTGTAAAGCCTGGGGTGCCTAAGTCGAC } \\
\text { GGATCCTTCAACTC }\end{array}$ \\
\hline C66 pBK_lambda_ConN25Prom_f & $\begin{array}{l}\text { GCGCAGGAAAGGTCTCACGGTTATAATGAGC } \\
\text { ACATAAATTTGAGAGAGGAGTTGTTTACGCT } \\
\text { TTGAGGAATC }\end{array}$ \\
\hline C67 pBK_lambdaProm_f & $\begin{array}{l}\text { GCGCAGGAAAGGTCTCACGGTGATACTGAGC } \\
\text { ACATCAGCAGGACGCACTGACCGTTTACGCT } \\
\text { TTGAGGAATC }\end{array}$ \\
\hline C68 pBK_lambdaProm_r & $\begin{array}{l}\text { GCGCAGAGTAGGTCTCAACCGCCAGTGGTAT } \\
\text { TTATGTCAACACCGCCAGAGATAAGTCGACG } \\
\text { GATCCTTCAACTC }\end{array}$ \\
\hline C69 pBK_T5DE20Prom_f & $\begin{array}{l}\text { GCGCAGGAAAGGTCTCAGCTTTAAGATGTAC } \\
\text { CCAGTTCGATGAGAGCGATAACGTTTACGCT } \\
\text { TTGAGGAATC }\end{array}$ \\
\hline C70 pBK_T5DE2OProm_r & $\begin{array}{l}\text { GCGCAGAGTAGGTCTCAAAGCCTATCGGCTA } \\
\text { GGGTGTCAAACTATTTTTGCAGTTGTCGACG } \\
\text { GATCCTTCAACTC }\end{array}$ \\
\hline C71 pBK_T5H207Prom_f & $\begin{array}{l}\text { GCGCAGGAAAGGTCTCACTCGTATAATATAC } \\
\text { TTCATAAATTGATAAACAAAAAGTTTACGCT } \\
\text { TTGAGGAATC }\end{array}$ \\
\hline C72 pBK_T5H207Prom_r & $\begin{array}{l}\text { GCGCAGAGTAGGTCTCACGAGAATTTGAAGC } \\
\text { GTTTAGCAAATGAATTTTTTAAAAGTCGACG } \\
\text { GATCCTTCAACTC }\end{array}$ \\
\hline C73 pBK_taclProm_f & $\begin{array}{l}\text { GCGCAGGAAAGGTCTCACTCGTATAATGTGT } \\
\text { GGAATTGTGAGCGGATAACAATGTTTACGCT } \\
\text { TTGAGGAATC }\end{array}$ \\
\hline C74 pBK_taclProm_r & $\begin{array}{l}\text { GCGCAGAGTAGGTCTCACGAGCCGATGATTA } \\
\text { ATTGTCAACAGCTCATTTCAGAAGTCGACGG } \\
\text { ATCCTTCAACTC }\end{array}$ \\
\hline C75 JYRS_2D12_Sall_f & $\begin{array}{l}\text { GAATTAATTGTCGACCCGCTTCGCAACATGT } \\
\text { GAG }\end{array}$ \\
\hline C76 JYRS_2D12_Sall_r & $\begin{array}{l}\text { GCGAAGCGGGTCGACAATTAATTCGCGAAGA } \\
\text { AAAG }\end{array}$ \\
\hline
\end{tabular}




\begin{tabular}{|c|c|}
\hline C77 PylS_H6L_Ndel_KpnI_f & $\begin{array}{l}\text { GAGGAATCCGGTACCATGGATAAAAAACCGC } \\
\text { TGGATG }\end{array}$ \\
\hline C78 PylS_H6L_Ndel_Kpnl_r & $\begin{array}{l}\text { TTTATCCATGGTACCGGATTCCTCAAAGCGT } \\
\text { AAAC }\end{array}$ \\
\hline C79 PylS_H6L_Pstl_Sacl_f & $\begin{array}{l}\text { AACCTGTAAGAGCTCTTTCAAACGCTAAATT } \\
\text { GCCTG }\end{array}$ \\
\hline C80 PylS_H6L_Pstl_Sacl_r & $\begin{array}{l}\text { CGTTTGAAAGAGCTCTTACAGGTTCGTGCTA } \\
\text { ATG }\end{array}$ \\
\hline C81 PylS_H6L_Sall_f & $\begin{array}{l}\text { GAAGGATCCGTCGACTCGGGTTGTCAGCCTG } \\
\text { TCCCGCTTATAAG }\end{array}$ \\
\hline C82 PylS_H6L_Sall_r & $\begin{array}{l}\text { ACAACCCGAGTCGACGGATCCTTCAACTCAG } \\
\text { CAAAAG }\end{array}$ \\
\hline C83 PylS_H6L_Notl_f & $\begin{array}{l}\text { ATTAATTGGCGGCCGCCCGCTTCGCACATGT } \\
\text { GAGCAAAAG }\end{array}$ \\
\hline C84 PylS_H6L_Notl_r & $\begin{array}{l}\text { CGAAGCGGGCGGCCGCCAATTAATTCGCGAA } \\
\text { GAAAAG }\end{array}$ \\
\hline C85 pREP_PylT_short_ORI_f & $\begin{array}{l}\text { TACTCTCGCATGGCCAGATGGTAAGCCCTCC } \\
\text { CGTATC }\end{array}$ \\
\hline C86 pREP_PylT_short_ORI_r & $\begin{array}{l}\text { TTACCATCTGGCCATGCGAGAGTAGGGAACT } \\
\text { GCCAG }\end{array}$ \\
\hline C87 pREP_PyIT_Notl_f & $\begin{array}{l}\text { AACGATCGGCGGCCGCAAAAATAAGTGGCGC } \\
\text { CCCATC }\end{array}$ \\
\hline C88 pREP_PylT_Notl_r & $\begin{array}{l}\text { TATTTTTGCGGCCGCCGATCGTTCGCTCAAA } \\
\text { GAAG }\end{array}$ \\
\hline C89 pREP_tRNA_seq_f & GAGGCCGCTCATGGCGTTC \\
\hline C90 pREP_PylT_Mfel_f & $\begin{array}{l}\text { GCCGTCGTTCAATTGTTACAACGTCGTGACT } \\
\text { GGGAAAAC }\end{array}$ \\
\hline C91 pREP_PylT_Mfel_r & $\begin{array}{l}\text { ACGTTGTAACAATTGAACGACGGCCAGTGCC } \\
\text { AAG }\end{array}$ \\
\hline C92 pREP_YCJYcua_AGGA2_Mfel_f & $\begin{array}{l}\text { GACTGTTGTCAATTGAACTCAGAATAAGAAA } \\
\text { TGAG }\end{array}$ \\
\hline C93 pREP_YCJYcua_AGGA2_Mfel_r & $\begin{array}{l}\text { TTCTGAGTTCAATTGACAACAGTCCGCACCG } \\
\text { CTGCCGGTAG }\end{array}$ \\
\hline C94 pREP_YCJYcua_AGGA2_Xhol_f & $\begin{array}{l}\text { GTCGTTTTACTCGAGCAACGTCGTGACTGGG } \\
\text { AAAAC }\end{array}$ \\
\hline C95 pREP_YCJYcua_AGGA2_Xhol_r & $\begin{array}{l}\text { ACGACGTTGCTCGAGTAAAACGACGGCCAGT } \\
\text { GCCAAG }\end{array}$ \\
\hline C96 PylS_Ndel-Restore_f & $\begin{array}{l}\text { GAGGAATCCCATATGATGGATAAAAAACCGC } \\
\text { TGGATG }\end{array}$ \\
\hline C97 PylS_Ndel-Restore_r & $\begin{array}{l}\text { TTTATCCATCATATGGGATTCCTCAAAGCGT } \\
\text { AAAC }\end{array}$ \\
\hline C98 PylS_Ndel-Restore_PM1_f & $\begin{array}{l}\text { GAGGAATCCTATATGATGGATAAAAAACCGC } \\
\text { TGGATG }\end{array}$ \\
\hline C99 PylS_Ndel-Restore_PM1_r & $\begin{array}{l}\text { TTTATCCATCATATAGGATTCCTCAAAGCGT } \\
\text { AAAC }\end{array}$ \\
\hline C100 PylS_Ndel-Restore_PM2_f & $\begin{array}{l}\text { GAGGAATCCCGTATGATGGATAAAAAACCGC } \\
\text { TGGATG }\end{array}$ \\
\hline C101 PylS_Ndel-Restore_PM2_r & $\begin{array}{l}\text { TTTATCCATCATACGGGATTCCTCAAAGCGT } \\
\text { AAAC }\end{array}$ \\
\hline C102 PylS_Ndel-Restore_PM3_f & $\begin{array}{l}\text { GAGGAATCCCATACGATGGATAAAAAACCGC } \\
\text { TGGATG }\end{array}$ \\
\hline C103 PylS_Ndel-Restore_PM3_r & $\begin{array}{l}\text { TTTATCCATCGTATGGGATTCCTCAAAGCGT } \\
\text { AAAC }\end{array}$ \\
\hline C104 PylS_Ndel-Restore_PM4_f & $\begin{array}{l}\text { GAGGAATCCCATATAATGGATAAAAAACCGC } \\
\text { TGGATG }\end{array}$ \\
\hline
\end{tabular}




\begin{tabular}{|c|c|}
\hline C105 PylS_Ndel-Restore_PM4_r & $\begin{array}{l}\text { TTTATCCATTATATGGGATTCCTCAAAGCGT } \\
\text { AAAC }\end{array}$ \\
\hline C106 PylS_Ndel-Restore_PM5_f & $\begin{array}{l}\text { GAGGAATCCTATATAATGGATAAAAAACCGC } \\
\text { TGGATG }\end{array}$ \\
\hline C107 PylS_Ndel-Restore_PM5_r & $\begin{array}{l}\text { TTTATCCATTATATAGGATTCCTCAAAGCGT } \\
\text { AAAC }\end{array}$ \\
\hline C108 T7seqf & TAATACGACTCACTATAGG \\
\hline C109 pCDF43_PylS_seqfII & CCAATGGATTTAAAAAATG \\
\hline C110 pCDF_4comp_seq1f & ATAGATTGGCTTTAAAAAC \\
\hline C111 pCDF_4comp_seq2f & AGATTTTAGAGCCAATTAG \\
\hline C112 pCDF_4comp_seq3f & AGATATCAACAACTTTCTG \\
\hline C113 pCDF_4comp_seq4f & CCTGTGCCTGCGTCCGATG \\
\hline C114 pCDF_4comp_seq5f & CGGCATTAGCACGAACCTG \\
\hline C115 ORBS_rep_dGST_uRS_f & $\begin{array}{l}\text { ACTAGCCATGGATCCAGGCGGCCGCTAAGCT } \\
\text { TAATTAGCTG }\end{array}$ \\
\hline C116 ORBS_rep_dGST_uRS_r & $\begin{array}{l}\text { CTTAGCGGCCGCCTGGATCCATGGCTAGTAT } \\
\text { AGGGGACATTTG }\end{array}$ \\
\hline C117 ORBS_rep_seq_f & CACTCATTAGGCACCCCAG \\
\hline C118 ORBS_rep_seq_r & TCACCGTCATCACCGAAAC \\
\hline C119 ORBS_rep_sfGFP_Ncol_f & $\begin{array}{l}\text { ATCACCATGGTTAGCAAAGGTGAAGAACTGT } \\
\text { TTAC }\end{array}$ \\
\hline C120 ORBS_rep_sfGFP_HindIII_r & $\begin{array}{l}\text { TGATAAGCTTAATGGTGATGATGATGGTGGC } \\
\text { TG }\end{array}$ \\
\hline C121 pCAT_T6TAG_f & $\begin{array}{l}\text { GAAAAAAATCTAGGGATATACCACCGTTGAT } \\
\text { ATATC }\end{array}$ \\
\hline C122 pCAT_T6TAG_r & $\begin{array}{l}\text { TGGTATATCCCTAGATTTTTTTCTCCATTTG } \\
\text { CGGAG }\end{array}$ \\
\hline C123 pCAT_D111AGGA_f & $\begin{array}{l}\text { TGAATACCACAGGAGATTTCCGGCAGTTTCT } \\
\text { ACAC }\end{array}$ \\
\hline C124 pCAT_D111AGGA_r & $\begin{array}{l}\text { GCCGGAAATCTCCTGTGGTATTCACTCCAGA } \\
\text { GCGATG }\end{array}$ \\
\hline C125 pCATseq1750rv & CAGCCTTTTTTCTCCTGCCAC \\
\hline C126 pCATseq1100rv & ATCTGCAGAATTCGCCCTTC \\
\hline C127 pCATseq350fw & CCAAAACAGCCAAGCTGGAC \\
\hline C128 CNPheRS_Ndel_f & $\begin{array}{l}\text { ATCACATATGGACGAATTTGAAATGATAAAG } \\
\text { AGAAAC }\end{array}$ \\
\hline C129 CNPheRS_PstI_r & $\begin{array}{l}\text { TGATCTGCAGTTATAATCTCTTTCTAATTGG } \\
\text { CTCTAAAATC }\end{array}$ \\
\hline C130 MjYRS23012NNK_for & $\begin{array}{l}\text { GTAGGTCTCGGCTAAGATAAAGAAAGCANNK } \\
\text { NNKNNKGCTGGAGTTGTTGAAGGAAATCCAA } \\
\text { TAATGGAGATAG }\end{array}$ \\
\hline C131 MjYRS2836NNK_for & $\begin{array}{l}\text { GTAGGTCTCATGAGGAGTTAGAGAGTTTATT } \\
\text { TAAAAATAAGGAATTGNNKCCAATGNNKTTA } \\
\text { AAAAATGCTGTAGCTGAAGAACTAAAAAG }\end{array}$ \\
\hline C132 MjYRS261MNN_rev & $\begin{array}{l}\text { GTAGGTCTCCCTCATAGCTATTAACTGTCAA } \\
\text { ATCTCCACCMNNTTTTTCTGGCCTTTTTATG } \\
\text { GTTAAAGGATATTC }\end{array}$ \\
\hline C133 H3opt_T6AGGA_f & $\begin{array}{l}\text { TACCAAACAGAGGAGCGCGTAAAAGCACCGG } \\
\text { CGGCAAAG }\end{array}$ \\
\hline C134 H3opt_T6AGGA_r & $\begin{array}{l}\text { TTTTACGCGCTCCTCTGTTTGGTACGGCCCT } \\
\text { GAAAATAC }\end{array}$ \\
\hline C135 ORBS_rep_H3_Ncol_f & $\begin{array}{l}\text { ATCACCATGGGCAGCAGCCATCACCATCATC } \\
\text { ACCACAG }\end{array}$ \\
\hline C136 ORBS_rep_H3optT6AGGA_HindIII_r & $\begin{array}{l}\text { TGATAAGCTTACGCACGTTCGCCACGAATAC } \\
\text { GAC }\end{array}$ \\
\hline
\end{tabular}




\begin{tabular}{|c|c|}
\hline C137 pACYC_Agel_f & $\begin{array}{l}\text { ATCAACCGGTAAACCAGCAATAGACATAAGC } \\
\text { GGCTATTTAAC }\end{array}$ \\
\hline C138 pACYC_Nhel_r & $\begin{array}{l}\text { TGATGCTAGCGCAGAATAAATAAATCCTGGT } \\
\text { GTCCCTG }\end{array}$ \\
\hline C139 pET_Agel_f & $\begin{array}{l}\text { ATCAACCGGTGGATCTTCACCTAGATCCTTT } \\
\text { TAAATTAAAAATG }\end{array}$ \\
\hline C140 pET_Nhel_upstream_r & $\begin{array}{l}\text { TGATGCTAGCTGTTTATTTTTCTAAATACAT } \\
\text { TCAAATATG }\end{array}$ \\
\hline C141 pCDF_resi_seqf & CTGAAACCTCAGGCATTTG \\
\hline C142 pCDF_resi_seqr & ACTTTGTATGTGTCCGCAG \\
\hline C143 ORBS_pBAD_GFP_f & $\begin{array}{l}\text { TGGGTTCATATCCCTCCGCAAATGGTTAGCA } \\
\text { AAGGTG }\end{array}$ \\
\hline C144 ORBS_pBAD_GFP_r & $\begin{array}{l}\text { CATTTGCGGAGGGATATGAACCCAAAAAAAC } \\
\text { GGGTATG }\end{array}$ \\
\hline C145 pBADseqf & ATTAGCGGATCCTACCTGAC \\
\hline C146 ORBS_pBAD_GCN4_f & $\begin{array}{l}\text { AATGAACTACCACCTGGAAAACGAAGTTGCG } \\
\text { CGTCTGAAAAAACTGGTTGGTTAAAGCTCGA } \\
\text { GATCTG }\end{array}$ \\
\hline C147 ORBS_pBAD_GCN4_r & $\begin{array}{l}\text { TTTAACCAACCAGTTTTTTCAGACGCGCAAC } \\
\text { TTCGTTTCCAGGTGGTAGTTCATTTGCGGA } \\
\text { GGGATATG }\end{array}$ \\
\hline
\end{tabular}




\subsection{Construction of Plasmids}

Table 8.3: Details of the construction of plasmids made during this thesis.

Plasmids that are listed in Table 8.1 and made during this thesis are aligned with the method used, primers (listed in Table 8.2), the template and the purpose of the mutagenesis. The corresponding sequencing primers (Seq. Primer) can also be found in the list. Methods: $Q C=$ Quik Change (Ch. 3.2.3.10); Inv = Inverse PCR (Ch. 3.2.3.11); Sub = Subcloning (Ch. 3.2.3.1 to 3.2.3.6).

\begin{tabular}{|c|c|c|c|c|c|}
\hline Product & Method & Primer & Template & Purpose & $\begin{array}{l}\text { Seq. } \\
\text { Primer }\end{array}$ \\
\hline pCLA7 & QC & $\mathrm{C} 1+\mathrm{C} 2$ & pCLA1 & $\mathrm{N}$-terminal $\mathrm{His}_{6}$-tag on PylS & $\mathrm{C} 3$ \\
\hline pCLA8 & QC & $\mathrm{C} 4+\mathrm{C} 5$ & pCLA1 & C-terminal $\mathrm{His}_{6}$-tag on PylS & $\mathrm{C} 3 / \mathrm{C6}$ \\
\hline pCLA9 & QC & $\mathrm{C} 7+\mathrm{C} 8$ & pCLA1 & $\begin{array}{l}\text { Internal } \mathrm{His}_{6} \text {-tag in the "gap" } \\
\text { between S144 and S145 on PyIS }\end{array}$ & $\mathrm{C} 3 / \mathrm{C6}$ \\
\hline pCLA10 & QC & $\mathrm{C} 9+\mathrm{C} 10$ & pCLA1 & $\begin{array}{l}\text { Internal } \mathrm{His}_{6} \text {-tag in the loop } \\
\text { D171 to F181 on PylS }\end{array}$ & $\mathrm{C} 3 / \mathrm{C6}$ \\
\hline pCLA11 & QC & $\mathrm{C} 11+\mathrm{C} 12$ & pCLA9 & $\begin{array}{l}\text { deletion of } 10 \text { AAs of the linker } \\
\text { region (in front of internal } \mathrm{His}_{6^{-}} \\
\text {tag) }\end{array}$ & $\mathrm{C} 3 / \mathrm{C6}$ \\
\hline pCLA12 & QC & $\mathrm{C} 13+\mathrm{C} 14$ & pCLA9 & $\begin{array}{l}\text { deletion of } 20 \text { AAs of the linker } \\
\text { region (in front of internal } \mathrm{His}_{6}- \\
\text { tag) }\end{array}$ & $\mathrm{C3} / \mathrm{C6}$ \\
\hline pCLA13 & QC & $\mathrm{C} 15+\mathrm{C} 16$ & pCLA9 & $\begin{array}{l}\text { deletion of } 15 \mathrm{AAs} \text { of the linker } \\
\text { region (behind internal } \mathrm{His}_{6} \text {-tag) }\end{array}$ & $\mathrm{C3} / \mathrm{C6}$ \\
\hline pCLA14 & QC & $\mathrm{C} 17+\mathrm{C} 18$ & pCLA9 & $\begin{array}{l}\text { deletion of } 30 \mathrm{AAs} \text { of the linker } \\
\text { region (behind internal } \mathrm{His}_{6} \text {-tag) }\end{array}$ & $\mathrm{C3} / \mathrm{C6}$ \\
\hline pCLA15 & QC & $\mathrm{C} 19+\mathrm{C} 20$ & pCLA9 & $\begin{array}{l}\text { deletion of } 50 \mathrm{AAs} \text { of the linker } \\
\text { region (in front of and behind } \\
\text { internal } \mathrm{His}_{6} \text {-tag) }\end{array}$ & $\mathrm{C3} / \mathrm{C6}$ \\
\hline pCLA16 & QC & $\mathrm{C} 21+\mathrm{C} 22$ & pCLA9 & $\begin{array}{l}\text { deletion of C-terminal part } \\
\text { beginning behind internal } \mathrm{His}_{6^{-}} \\
\text {tag }\end{array}$ & $\mathrm{C3} / \mathrm{C6}$ \\
\hline pCLA17 & QC & $\mathrm{C} 23+\mathrm{C} 24$ & pCLA9 & $\begin{array}{l}\text { deletion of } \mathrm{N} \text {-terminal part up } \\
\text { to internal His-Tag }\end{array}$ & $\mathrm{C3} / \mathrm{C6}$ \\
\hline pCLA18 & QC & $\mathrm{C} 34+\mathrm{C} 35$ & $\mathrm{pCLA2}$ & $\begin{array}{l}\text { N-terminal } \mathrm{His}_{6} \text {-tag on } \\
\text { MjYRS_TAG }\end{array}$ & $\mathrm{C3}$ \\
\hline pCLA19 & QC & $\mathrm{C} 36+\mathrm{C} 37$ & pCLA2 & $\begin{array}{l}\text { C-terminal } \mathrm{His}_{6} \text {-tag on } \\
\text { MjYRS_TAG }\end{array}$ & $\mathrm{C} 3 / \mathrm{C6}$ \\
\hline pCLA20 & QC & C38+C39 & $\mathrm{pCLA2}$ & $\begin{array}{l}\text { Internal } \mathrm{His}_{6} \text {-tag in the loop } \mathrm{K} 25 \\
\text { to } \mathrm{K} 29 \text { on MjYRS_TAG }\end{array}$ & $\mathrm{C3} / \mathrm{C6}$ \\
\hline pCLA22 & $\operatorname{lnv}$ & $\mathrm{C} 48+\mathrm{C} 49$ & pCLA86 & PyIS promoter library & C6/C50 \\
\hline pCLA23 & $\operatorname{lnv}$ & $\mathrm{C} 48+\mathrm{C} 49$ & pCLA86 & PyIS promoter library & C6/C50 \\
\hline pCLA24 & $\operatorname{lnv}$ & $\mathrm{C} 48+\mathrm{C} 49$ & pCLA86 & PyIS promoter library & C6/C50 \\
\hline pCLA25 & $\ln v$ & $\mathrm{C} 48+\mathrm{C} 49$ & pCLA86 & PyIS promoter library & C6/C50 \\
\hline pCLA26 & $\operatorname{lnv}$ & $\mathrm{C} 48+\mathrm{C} 49$ & pCLA86 & PyIS promoter library & C6/C50 \\
\hline pCLA27 & $\operatorname{lnv}$ & $\mathrm{C} 48+\mathrm{C} 49$ & pCLA86 & PyIS promoter library & C6/C50 \\
\hline pCLA28 & $\operatorname{lnv}$ & $\mathrm{C} 48+\mathrm{C} 49$ & pCLA86 & PyIS promoter library & C6/C50 \\
\hline pCLA29 & $\ln v$ & $\mathrm{C} 48+\mathrm{C} 49$ & pCLA86 & PyIS promoter library & C6/C50 \\
\hline pCLA30 & $\ln v$ & $\mathrm{C} 48+\mathrm{C} 49$ & pCLA86 & PylS promoter library & C6/C50 \\
\hline pCLA31 & Inv & $\mathrm{C} 48+\mathrm{C} 49$ & pCLA86 & Pyls promoter library & C6/C50 \\
\hline pCLA33 & $\operatorname{lnv}$ & $\mathrm{C} 48+\mathrm{C} 51$ & pCLA72 & MjYRS_AGGA promoter library & $\mathrm{C} 52 / \mathrm{C} 53$ \\
\hline pCLA34 & $\operatorname{lnv}$ & $\mathrm{C} 48+\mathrm{C} 51$ & pCLA72 & MjYRS_AGGA promoter library & C52/C53 \\
\hline pCLA35 & Inv & $\mathrm{C} 48+\mathrm{C} 51$ & pCLA72 & MjYRS_AGGA promoter library & C52/C53 \\
\hline
\end{tabular}




\begin{tabular}{|c|c|c|c|c|c|}
\hline pCLA36 & Inv & $\mathrm{C} 48+\mathrm{C} 51$ & pCLA72 & MjYRS_AGGA promoter library & C52/C53 \\
\hline pCLA37 & $\operatorname{lnv}$ & $\mathrm{C} 48+\mathrm{C} 51$ & pCLA72 & MjYRS_AGGA promoter library & $\mathrm{C} 52 / \mathrm{C} 53$ \\
\hline pCLA38 & $\ln v$ & $\mathrm{C} 48+\mathrm{C} 51$ & pCLA72 & MjYRS_AGGA promoter library & C52/C53 \\
\hline pCLA39 & $\operatorname{lnv}$ & $\mathrm{C} 48+\mathrm{C} 51$ & pCLA72 & MjYRS_AGGA promoter library & C52/C53 \\
\hline pCLA40 & $\operatorname{lnv}$ & $\mathrm{C} 48+\mathrm{C} 51$ & pCLA72 & MjYRS_AGGA promoter library & C52/C53 \\
\hline pCLA41 & $\operatorname{lnv}$ & $\mathrm{C} 48+\mathrm{C} 51$ & pCLA72 & MjYRS_AGGA promoter library & C52/C53 \\
\hline pCLA42 & $\ln v$ & $\mathrm{C} 48+\mathrm{C} 51$ & pCLA72 & MjYRS_AGGA promoter library & C52/C53 \\
\hline pCLA44 & $\operatorname{lnv}$ & $\mathrm{C} 54+\mathrm{C} 55$ & pCLA79 & PylT promoter library & C57 \\
\hline pCLA45 & $\ln v$ & $\mathrm{C} 54+\mathrm{C} 55$ & pCLA79 & PylT promoter library & C57 \\
\hline pCLA46 & $\ln v$ & $\mathrm{C} 54+\mathrm{C} 55$ & pCLA79 & PylT promoter library & C57 \\
\hline pCLA47 & $\operatorname{lnv}$ & $\mathrm{C} 54+\mathrm{C} 55$ & pCLA79 & PylT promoter library & C57 \\
\hline pCLA48 & $\ln v$ & C54 + C55 & pCLA79 & PylT promoter library & C57 \\
\hline pCLA49 & $\operatorname{lnv}$ & $\mathrm{C} 54+\mathrm{C} 55$ & pCLA79 & PylT promoter library & C57 \\
\hline pCLA50 & $\ln v$ & $\mathrm{C} 54+\mathrm{C} 55$ & pCLA79 & PylT promoter library & C57 \\
\hline pCLA51 & $\ln v$ & C54 + C55 & pCLA79 & PylT promoter library & C57 \\
\hline pCLA52 & $\operatorname{lnv}$ & $\mathrm{C} 54+\mathrm{C} 55$ & pCLA79 & PylT promoter library & C57 \\
\hline pCLA53 & $\ln v$ & $\mathrm{C} 56+\mathrm{C} 55$ & pCLA82 & MjYT_UCCU promoter library & C57 \\
\hline pCLA54 & $\operatorname{lnv}$ & $\mathrm{C} 56+\mathrm{C} 55$ & pCLA82 & MjYT_UCCU promoter library & C57 \\
\hline pCLA55 & $\ln v$ & $\mathrm{C} 56+\mathrm{C} 55$ & pCLA82 & MjYT_UCCU promoter library & C57 \\
\hline pCLA56 & $\ln v$ & $\mathrm{C} 56+\mathrm{C} 55$ & pCLA82 & MjYT_UCCU promoter library & C57 \\
\hline pCLA57 & Inv & $\mathrm{C} 56+\mathrm{C} 55$ & pCLA82 & MjYT_UCCU promoter library & C57 \\
\hline pCLA58 & $\ln v$ & $\mathrm{C} 56+\mathrm{C} 55$ & pCLA82 & MjYT_UCCU promoter library & C57 \\
\hline pCLA59 & $\operatorname{lnv}$ & $\mathrm{C} 56+\mathrm{C} 55$ & pCLA82 & MjYT_UCCU promoter library & C57 \\
\hline pCLA60 & $\operatorname{lnv}$ & $\mathrm{C} 56+\mathrm{C} 55$ & pCLA82 & MjYT_UCCU promoter library & C57 \\
\hline pCLA61 & $\ln v$ & C56 + C55 & pCLA82 & MjYT_UCCU promoter library & C57 \\
\hline pCLA62 & $\ln v$ & C58 + C59 & pCLA85 & $\begin{array}{l}\text { gInS promoter in front of } p y / S \\
\text { exchanged for } \mathrm{P}_{\text {con }}\end{array}$ & C50/C53 \\
\hline pCLA63 & $\ln v$ & $\mathrm{C} 60+\mathrm{C} 61$ & pCLA85 & $\begin{array}{l}\text { gInS promoter in front of } p y / S \\
\text { exchanged for } \mathrm{P}_{\mathrm{cp} 25}\end{array}$ & C50/C53 \\
\hline pCLA64 & $\ln v$ & $\mathrm{C} 62+\mathrm{C} 63$ & pCLA85 & $\begin{array}{l}\text { gInS promoter in front of } p y / S \\
\text { exchanged for } \mathrm{P}_{\text {lac1- } 6 \mathrm{Mt} 5}\end{array}$ & C50/C53 \\
\hline pCLA65 & $\ln v$ & $\mathrm{C} 64+\mathrm{C} 65$ & pCLA85 & $\begin{array}{l}\text { gInS promoter in front of } p y / S \\
\text { exchanged for } \mathrm{P}_{\mathrm{lac}}\end{array}$ & C50/C53 \\
\hline pCLA66 & $\ln v$ & $\mathrm{C} 66+\mathrm{C68}$ & pCLA85 & $\begin{array}{l}\text { glnS promoter in front of } p y / S \\
\text { exchanged for } \mathrm{P}_{\lambda \text { con/N25DSR }}\end{array}$ & C50/C53 \\
\hline pCLA67 & $\ln v$ & $\mathrm{C} 67+\mathrm{C} 68$ & pCLA85 & $\begin{array}{l}\text { gInS promoter in front of } p y / S \\
\text { exchanged for } \mathrm{P}_{\lambda}\end{array}$ & $\mathrm{C} 50 / \mathrm{C} 53$ \\
\hline pCLA68 & $\ln v$ & $\mathrm{C} 69+\mathrm{C} 70$ & pCLA85 & $\begin{array}{l}\text { glnS promoter in front of } p y / S \\
\text { exchanged for } \mathrm{P}_{\mathrm{D} / \mathrm{E} 20}\end{array}$ & C50/C53 \\
\hline pCLA69 & $\ln v$ & $\mathrm{C} 71+\mathrm{C} 72$ & pCLA85 & $\begin{array}{l}\text { glnS promoter in front of } p y / S \\
\text { exchanged for } \mathrm{P}_{\mathrm{H} 207}\end{array}$ & C50/C53 \\
\hline pCLA70 & $\ln v$ & $\mathrm{C} 73+\mathrm{C} 74$ & pCLA85 & $\begin{array}{l}\text { glnS promoter in front of } p y / S \\
\text { exchanged for } \mathrm{P}_{\mathrm{tacl}}\end{array}$ & C50/C53 \\
\hline pCLA71 & QC & C36+C37 & pCLA3 & $\begin{array}{l}\text { C-terminal His } \text {-tag on }_{6} \\
\text { MjYRS_AGGA }\end{array}$ & C3/C6 \\
\hline pCLA72 & QC & $\mathrm{C} 75+\mathrm{C} 76$ & pCLA71 & $\begin{array}{l}\text { Sall restriction site behind } \\
\text { MjYRS terminator }\end{array}$ & C3/C53 \\
\hline pCLA73 & QC & $\mathrm{C} 77+\mathrm{C} 78$ & pCLA9 & $\begin{array}{l}\text { Ndel restriction site in front of } \\
\text { PylS start codon changed to Kpnl }\end{array}$ & $\mathrm{C3} / \mathrm{C6}$ \\
\hline pCLA74 & QC & $\mathrm{C} 79+\mathrm{C} 80$ & pCLA73 & $\begin{array}{l}\text { Pstl restriction site behind PylS } \\
\text { stop codon changed to Sacl }\end{array}$ & C50/C53 \\
\hline
\end{tabular}




\begin{tabular}{|c|c|c|c|c|c|}
\hline pCLA75 & QC & $\mathrm{C} 81+\mathrm{C} 82$ & pCLA74 & $\begin{array}{l}\text { Sall restriction site in front of } \\
\text { PylS promoter }\end{array}$ & C6/C50 \\
\hline pCLA76 & QC & $\mathrm{C} 83+\mathrm{C} 84$ & pCLA75 & $\begin{array}{l}\text { Notl restriction site behind PyIS } \\
\text { terminator }\end{array}$ & C50/C53 \\
\hline pCLA77 & QC & $\mathrm{C} 85+\mathrm{C} 86$ & pCLA4 & $\begin{array}{l}\text { deletion of genes for T7 RNA } \\
\text { Polymerase, AraC and GFP }\end{array}$ & digest only \\
\hline pCLA78 & QC & $\mathrm{C} 87+\mathrm{C} 88$ & pCLA77 & $\begin{array}{l}\text { Notl restriction site in front of } \\
\text { PylT promoter }\end{array}$ & C89 \\
\hline pCLA79 & QC & $\mathrm{C} 90+\mathrm{C} 91$ & pCLA78 & $\begin{array}{l}\text { Mfel restriction site behind PylT } \\
\text { terminator }\end{array}$ & C89 \\
\hline pCLA80 & QC & $\mathrm{C} 85+\mathrm{C} 86$ & pCLA6 & $\begin{array}{l}\text { deletion of genes for T7 RNA } \\
\text { Polymerase, AraC and GFP }\end{array}$ & digest only \\
\hline pCLA81 & QC & $\mathrm{C} 92+\mathrm{C93}$ & pCLA80 & $\begin{array}{l}\text { Mfel restriction site in front of } \\
\text { MjYT_UCCU promoter }\end{array}$ & C57 \\
\hline pCLA82 & QC & $\mathrm{C} 94+\mathrm{C} 95$ & pCLA81 & $\begin{array}{l}\text { Xhol restriction site behind } \\
\text { MjYT_UCCU terminator }\end{array}$ & C57/C89 \\
\hline pCLA83 & QC & $\mathrm{C} 81+\mathrm{C} 82$ & pCLA9 & $\begin{array}{l}\text { Sall restriction site in front of } \\
\text { PylS promoter }\end{array}$ & C6/C50 \\
\hline pCLA84 & QC & $\mathrm{C} 83+\mathrm{C} 84$ & pCLA9 & $\begin{array}{l}\text { Notl restriction site behind PyIS } \\
\text { terminator }\end{array}$ & C50/C53 \\
\hline pCLA85 & QC & $\mathrm{C} 96+\mathrm{C} 97$ & pCLA76 & Ndel site on pCLA76 restored & C50/C53 \\
\hline pCLA86 & QC & $\mathrm{C} 98+\mathrm{C99}$ & pCLA76 & $\begin{array}{l}\text { point mutation in restored Ndel } \\
\text { site }\end{array}$ & C50/C53 \\
\hline pCLA87 & QC & C100 + C101 & pCLA76 & $\begin{array}{l}\text { point mutation in restored Ndel } \\
\text { site }\end{array}$ & C50/C53 \\
\hline pCLA88 & QC & $\mathrm{C} 102+\mathrm{C} 103$ & pCLA76 & $\begin{array}{l}\text { point mutation in restored Ndel } \\
\text { site }\end{array}$ & C50/C53 \\
\hline pCLA89 & QC & C104 + C105 & pCLA76 & $\begin{array}{l}\text { point mutation in restored Ndel } \\
\text { site }\end{array}$ & C50/C53 \\
\hline pCLA90 & QC & C106 + C107 & pCLA76 & $\begin{array}{l}\text { point mutation in restored Ndel } \\
\text { site }\end{array}$ & C50/C53 \\
\hline pCLA92 & Sub & - & pCLA72 & $\begin{array}{l}\text { MjYRS_AGGA gene cloned with } \\
\text { BamHI + Sall into pCLA91 }\end{array}$ & $\begin{array}{l}\mathrm{C} 79 / \mathrm{C} 89 / \\
\mathrm{C} 108-\mathrm{C} 114\end{array}$ \\
\hline pCLA93 & Sub & - & pCLA86 & $\begin{array}{l}\text { PylS gene cloned with Sall + } \\
\text { Notl (+ Xhol) into pCLA92 }\end{array}$ & $\begin{array}{l}\mathrm{C} 79 / \mathrm{C} 89 / \\
\mathrm{C} 108-\mathrm{C} 114\end{array}$ \\
\hline pCLA94 & Sub & - & pCLA79 & $\begin{array}{l}\text { PylT gene cloned with Notl + } \\
\text { Mfel into pCLA93 }\end{array}$ & $\begin{array}{l}\mathrm{C} 79 / \mathrm{C} 89 / \\
\mathrm{C} 108-\mathrm{C} 114\end{array}$ \\
\hline pCLA95 & Sub & - & pCLA82 & $\begin{array}{l}\text { MjYT_UCCU gene cloned with } \\
\text { Mfel + Xhol into pCLA94 }\end{array}$ & $\begin{array}{l}\mathrm{C} 79 / \mathrm{C} 89 / \\
\mathrm{C} 108-\mathrm{C} 114\end{array}$ \\
\hline pCLA100 & QC & $\mathrm{C} 115+\mathrm{C} 116$ & pCLA99 & $\begin{array}{l}\text { deletion of the GST-MBP gene } \\
\text { and incorporation of a MCS }\end{array}$ & C117/C118 \\
\hline pCLA105 & Sub & $\mathrm{C} 119+\mathrm{C} 120$ & pCLA101 & $\begin{array}{l}\text { sfGFP WT gene cloned with Ncol } \\
+ \text { Hindlll into pCLA100 }\end{array}$ & C117/C118 \\
\hline pCLA106 & Sub & $\mathrm{C} 119+\mathrm{C} 120$ & pCLA102 & $\begin{array}{l}\text { sfGFP 150TAG gene cloned with } \\
\text { Ncol + Hindlll into pCLA100 }\end{array}$ & C117/C118 \\
\hline pCLA107 & Sub & $\mathrm{C} 119+\mathrm{C} 120$ & pCLA103 & $\begin{array}{l}\text { sfGFP 134AGGA 150TAG gene } \\
\text { cloned with Ncol + HindIII into } \\
\text { pCLA100 }\end{array}$ & C117/C118 \\
\hline pCLA108 & Sub & C119+ C120 & pCLA104 & $\begin{array}{l}\text { sfGFP 134AGGA gene cloned } \\
\text { with Ncol + HindIII into pCLA100 }\end{array}$ & C117/C118 \\
\hline pCLA112 & QC & $\mathrm{C} 121+\mathrm{C} 122$ & pCLA111 & $\begin{array}{l}\text { Codon for T6 in the CAT gene } \\
\text { exchanged for TAG }\end{array}$ & 5-C127 \\
\hline
\end{tabular}




\begin{tabular}{|c|c|c|c|c|c|}
\hline pCLA113 & QC & $\mathrm{C} 123+\mathrm{C} 124$ & pCLA112 & $\begin{array}{l}\text { Codon for D111 in the CAT gene } \\
\text { exchanged for AGGA }\end{array}$ & C125-C127 \\
\hline pCLA115 & Sub & - & pCLA24 & $\begin{array}{l}\text { py/S with } \mathrm{P}_{\text {glns }} \text { exchanged for } \\
\text { py/S with } \mathrm{P}_{\text {lib3E }} \text { by cloning into } \\
\text { pCLA95 with Sall + Notl (+ Xhol) }\end{array}$ & C111 \\
\hline pCLA116 & Sub & - & pCLA62 & $\begin{array}{l}\text { pylS with } \mathrm{P}_{\text {glns }} \text { exchanged for } \\
\text { pylS with } \mathrm{P}_{\text {con }} \text { by cloning into } \\
\text { pCLA95 with Sall + Notl (+ Xhol) }\end{array}$ & C111 \\
\hline pCLA117 & Sub & - & pCLA65 & $\begin{array}{l}\text { pylS with } \mathrm{P}_{\mathrm{glns}} \text { exchanged for } \\
\text { pylS with } \mathrm{P}_{\text {lac }} \text { by cloning into } \\
\text { pCLA95 with Sall + Notl (+ Xhol) }\end{array}$ & C111 \\
\hline pCLA120 & Sub & $\mathrm{C} 128+\mathrm{C} 129$ & pCLA119 & $\begin{array}{l}\text { MjAzFRS gene cloned with Ndel } \\
+ \text { Pstl into pCLA1 }\end{array}$ & $\mathrm{C} 3 / \mathrm{C} 6$ \\
\hline pCLA121 & $\ln v$ & $\begin{array}{l}\text { C130 and } \\
\text { C131 + C132 }\end{array}$ & pCLA120 & $\begin{array}{l}\text { MjAzFRS library in two rounds } \\
\text { of inverse PCR }\end{array}$ & C6 \\
\hline pCLA122 & $\ln v$ & $\begin{array}{l}\mathrm{C} 130 \text { and } \\
\mathrm{C} 131+\mathrm{C} 132\end{array}$ & pCLA120 & $\begin{array}{l}\text { MjAzFRS library in two rounds } \\
\text { of inverse PCR }\end{array}$ & C6 \\
\hline pCLA123 & $\ln v$ & $\begin{array}{l}\mathrm{C} 130 \text { and } \\
\mathrm{C} 131+\mathrm{C} 132\end{array}$ & pCLA120 & $\begin{array}{l}\text { MjAzFRS library in two rounds } \\
\text { of inverse PCR }\end{array}$ & C6 \\
\hline pCLA124 & $\ln v$ & $\begin{array}{l}\mathrm{C} 130 \text { and } \\
\mathrm{C} 131+\mathrm{C} 132 \\
\end{array}$ & $\mathrm{pCLA} 120$ & $\begin{array}{l}\text { MjAzFRS library in two rounds } \\
\text { of inverse PCR }\end{array}$ & C6 \\
\hline pCLA125 & $\ln v$ & $\begin{array}{l}\mathrm{C} 130 \text { and } \\
\mathrm{C} 131+\mathrm{C} 132\end{array}$ & pCLA120 & $\begin{array}{l}\text { MjAzFRS library in two rounds } \\
\text { of inverse PCR }\end{array}$ & C6 \\
\hline pCLA126 & $\ln v$ & $\begin{array}{l}\mathrm{C} 130 \text { and } \\
\mathrm{C} 131+\mathrm{C} 132 \\
\end{array}$ & pCLA120 & $\begin{array}{l}\text { MjAzFRS library in two rounds } \\
\text { of inverse PCR }\end{array}$ & C6 \\
\hline pCLA127 & $\ln v$ & $\begin{array}{l}\text { C130 and } \\
\mathrm{C} 131+\mathrm{C} 132 \\
\end{array}$ & pCLA120 & $\begin{array}{l}\text { MjAzFRS library in two rounds } \\
\text { of inverse PCR }\end{array}$ & C6 \\
\hline pCLA128 & $\ln v$ & $\begin{array}{l}\mathrm{C} 130 \text { and } \\
\mathrm{C} 131+\mathrm{C} 132\end{array}$ & pCLA120 & $\begin{array}{l}\text { MjAzFRS library in two rounds } \\
\text { of inverse PCR }\end{array}$ & C6 \\
\hline pCLA129 & $\ln v$ & $\begin{array}{l}\mathrm{C} 130 \text { and } \\
\mathrm{C} 131+\mathrm{C} 132\end{array}$ & pCLA120 & $\begin{array}{l}\text { MjAzFRS library in two rounds } \\
\text { of inverse PCR }\end{array}$ & C6 \\
\hline pCLA130 & $\ln v$ & $\begin{array}{l}\mathrm{C} 130 \text { and } \\
\mathrm{C} 131+\mathrm{C} 132\end{array}$ & pCLA120 & $\begin{array}{l}\text { MjAzFRS library in two rounds } \\
\text { of inverse PCR }\end{array}$ & C6 \\
\hline pCLA131 & $\ln v$ & $\begin{array}{l}\mathrm{C} 130 \text { and } \\
\mathrm{C} 131+\mathrm{C} 132\end{array}$ & pCLA120 & $\begin{array}{l}\text { MjAzFRS library in two rounds } \\
\text { of inverse PCR }\end{array}$ & C6 \\
\hline pCLA132 & $\ln v$ & $\begin{array}{l}\mathrm{C} 130 \text { and } \\
\mathrm{C} 131+\mathrm{C} 132\end{array}$ & pCLA120 & $\begin{array}{l}\text { MjAzFRS library in two rounds } \\
\text { of inverse PCR }\end{array}$ & C6 \\
\hline pCLA133 & $\ln v$ & $\begin{array}{l}\mathrm{C} 130 \text { and } \\
\mathrm{C} 131+\mathrm{C} 132\end{array}$ & pCLA120 & $\begin{array}{l}\text { MjAzFRS library in two rounds } \\
\text { of inverse PCR }\end{array}$ & C6 \\
\hline pCLA134 & $\ln v$ & $\begin{array}{l}\mathrm{C} 130 \text { and } \\
\mathrm{C} 131+\mathrm{C} 132\end{array}$ & pCLA120 & $\begin{array}{l}\text { MjAzFRS library in two rounds } \\
\text { of inverse PCR }\end{array}$ & C6 \\
\hline pCLA135 & $\ln v$ & $\begin{array}{l}\mathrm{C} 130 \text { and } \\
\mathrm{C} 131+\mathrm{C} 132\end{array}$ & pCLA120 & $\begin{array}{l}\text { MjAzFRS library in two rounds } \\
\text { of inverse PCR }\end{array}$ & C6 \\
\hline pCLA136 & $\operatorname{lnv}$ & $\begin{array}{l}\text { C130 and } \\
\text { C131 + C132 }\end{array}$ & pCLA120 & $\begin{array}{l}\text { MjAzFRS library in two rounds } \\
\text { of inverse PCR }\end{array}$ & C6 \\
\hline pCLA137 & $\ln v$ & $\begin{array}{l}\text { C130 and } \\
\mathrm{C} 131+\mathrm{C} 132\end{array}$ & pCLA120 & $\begin{array}{l}\text { MjAzFRS library in two rounds } \\
\text { of inverse PCR }\end{array}$ & C6 \\
\hline pCLA138 & $\ln v$ & $\begin{array}{l}\mathrm{C} 130 \text { and } \\
\mathrm{C} 131+\mathrm{C} 132\end{array}$ & pCLA120 & $\begin{array}{l}\text { MjAzFRS library in two rounds } \\
\text { of inverse PCR }\end{array}$ & C6 \\
\hline pCLA139 & $\ln v$ & $\begin{array}{l}\mathrm{C} 130 \text { and } \\
\mathrm{C} 131+\mathrm{C} 132\end{array}$ & pCLA120 & $\begin{array}{l}\text { MjAzFRS library in two rounds } \\
\text { of inverse PCR }\end{array}$ & C6 \\
\hline
\end{tabular}




\begin{tabular}{|c|c|c|c|c|c|}
\hline pCLA140 & $\ln v$ & $\begin{array}{l}\mathrm{C} 130 \text { and } \\
\mathrm{C} 131+\mathrm{C} 132\end{array}$ & pCLA120 & $\begin{array}{l}\text { MjAzFRS library in two rounds } \\
\text { of inverse PCR }\end{array}$ & C6 \\
\hline pCLA142 & QC & $\mathrm{C} 133+\mathrm{C} 134$ & pCLA141 & $\begin{array}{l}\text { Codon for T6 in the H3opt gene } \\
\text { exchanged for AGGA }\end{array}$ & C108 \\
\hline pCLA143 & Sub & $\mathrm{C} 135+\mathrm{C} 136$ & pCLA142 & $\begin{array}{l}\text { H3opt T6AGGA gene cloned with } \\
\text { Ncol + HindIII into pCLA100 }\end{array}$ & C117 \\
\hline pCLA144 & Sub & - & pCLA122 & $\begin{array}{l}\text { MjYRS gene on pCLA98 replaced } \\
\text { by MjAzFRS gene (clone \#2) with } \\
\text { Ndel + Stul }\end{array}$ & digest only \\
\hline pCLA145 & Sub & - & pCLA123 & $\begin{array}{l}\text { MjYRS gene on pCLA98 replaced } \\
\text { by MjAzFRS gene (clone \#3) with } \\
\text { Ndel + Stul }\end{array}$ & digest only \\
\hline pCLA146 & Sub & - & pCLA125 & $\begin{array}{l}\text { MjYRS gene on pCLA98 replaced } \\
\text { by MjAzFRS gene (clone \#5) with } \\
\text { Ndel + Stul }\end{array}$ & digest only \\
\hline pCLA147 & Sub & - & pCLA126 & $\begin{array}{l}\text { MjYRS gene on pCLA98 replaced } \\
\text { by MjAzFRS gene (clone \#6) with } \\
\text { Ndel + Stul }\end{array}$ & digest only \\
\hline pCLA148 & Sub & - & pCLA131 & $\begin{array}{l}\text { MjYRS gene on pCLA98 replaced } \\
\text { by MjAzFRS gene (clone \#11) } \\
\text { with Ndel + Stul }\end{array}$ & digest only \\
\hline pCLA149 & Sub & - & pCLA137 & $\begin{array}{l}\text { MjYRS gene on pCLA98 replaced } \\
\text { by MjAzFRS gene (clone \#17) } \\
\text { with Ndel + Stul }\end{array}$ & digest only \\
\hline pCLA150 & Sub & - & pCLA138 & $\begin{array}{l}\text { MjYRS gene on pCLA98 replaced } \\
\text { by MjAzFRS gene (clone \#18) } \\
\text { with Ndel + Stul }\end{array}$ & digest only \\
\hline pCLA152 & Sub & - & pCLA151 & $\begin{array}{l}\text { MjAzFRS (TAG) gene cloned into } \\
\text { pCLA91 with BamHI + Sall }\end{array}$ & $\mathrm{C3} / \mathrm{C6}$ \\
\hline pCLA153 & Sub & - & pCLA95 & $\begin{array}{l}\text { tRNA cassette (PylT and } \\
\text { MjYT_UCCU) cloned into } \\
\text { pCLA152 with Notl + Xhol }\end{array}$ & C89/C109 \\
\hline pCLA154 & Sub & - & pCLA137 & $\begin{array}{l}\text { MjAzFRS (TAG) gene on pCLA153 } \\
\text { replaced by MjAzFRS (AGGA, } \\
\text { clone \#17) with Ndel + Stul }\end{array}$ & $\mathrm{C3} / \mathrm{C6}$ \\
\hline pCLA155 & Sub & - & pCLA95 & $\begin{array}{l}\text { PylS gene cloned into pCLA154 } \\
\text { with Sall + Notl }\end{array}$ & C111-C113 \\
\hline pCLA165 & Sub & $\mathrm{C} 81+\mathrm{C} 84$ & pCLA158 & $\begin{array}{l}\text { BCNRS gene cloned into pCLA154 } \\
\text { with Sall + Notl }\end{array}$ & C111-C113 \\
\hline pCLA166 & Sub & $\mathrm{C} 81+\mathrm{C} 84$ & pCLA160 & $\begin{array}{l}\text { AzpcKRS gene cloned into } \\
\text { pCLA154 with Sall + Notl }\end{array}$ & C111-C113 \\
\hline pCLA167 & Sub & - & pCLA158 & $\begin{array}{l}\text { PylS gene on pCLA62 replaced by } \\
\text { BCNRS gene with Ndel + Stul }\end{array}$ & C6/C50 \\
\hline pCLA168 & Sub & - & pCLA158 & $\begin{array}{l}\text { PylS gene on pCLA65 replaced by } \\
\text { BCNRS gene with Ndel + Stul }\end{array}$ & C6/C50 \\
\hline pCLA169 & Sub & - & pCLA160 & $\begin{array}{l}\text { PylS gene on pCLA62 replaced by } \\
\text { AzpcKRS gene with Ndel + Stul }\end{array}$ & C6/C50 \\
\hline pCLA170 & Sub & - & pCLA160 & $\begin{array}{l}\text { PylS gene on pCLA65 replaced by } \\
\text { AzpcKRS gene with Ndel + Stul }\end{array}$ & C6/C50 \\
\hline pCLA171 & Sub & - & pCLA167 & $\begin{array}{l}\text { BCNRS gene with } P_{\text {con }} \text { cloned into } \\
\text { pCLA154 with Sall }+ \text { Notl }(+ \text { Xhol) }\end{array}$ & C111-C114 \\
\hline
\end{tabular}




\begin{tabular}{|l|l|c|l|l|l|}
\hline pCLA172 & Sub & - & pCLA168 & $\begin{array}{l}\text { BCNRS gene with Plac cloned into } \\
\text { pCLA154 with Sall + Notl + Xhol) }\end{array}$ & C111-C114 \\
\hline pCLA173 & Sub & - & pCLA169 & $\begin{array}{l}\text { AzpcKRS gene with P con cloned } \\
\text { into pCLA154 with Sall + Notl } \\
\text { (+ Xhol) }\end{array}$ & C111-C114 \\
\hline pCLA174 & Sub & - & pCLA170 & $\begin{array}{l}\text { AzpcKRS gene with Plac cloned } \\
\text { into pCLA154 with Sall + Notl } \\
\text { + Xhol) }\end{array}$ & C111-C114 \\
\hline pCLA184 & Sub & C137 + C138 & pCLA182 & $\begin{array}{l}\text { SmR gene on pCLA116 replaced } \\
\text { by CatR gene with Agel + Nhel }\end{array}$ & $\begin{array}{l}\text { C111/ } \\
\text { C141/C142 }\end{array}$ \\
\hline pCLA185 & Sub & C139 + C140 & pCLA183 & $\begin{array}{l}\text { SmR gene on pCLA116 replaced } \\
\text { by AmpR gene with Agel + Nhel }\end{array}$ & $\begin{array}{l}\text { C111/ } \\
\text { C141/C142 }\end{array}$ \\
\hline pCLA186 & Sub & C137+ C138 & pCLA182 & $\begin{array}{l}\text { SmR gene on pCLA171 replaced } \\
\text { by CatR gene with Agel + Nhel }\end{array}$ & $\begin{array}{l}\text { C111/ } \\
\text { C141/C142 }\end{array}$ \\
\hline pCLA187 & Sub & C139 + C140 & pCLA183 & $\begin{array}{l}\text { SmR gene on pCLA171 replaced } \\
\text { by AmpR gene with Agel + Nhel }\end{array}$ & $\begin{array}{l}\text { C111/ } \\
\text { C141/C142 }\end{array}$ \\
\hline pCLA193 & QC & C143 + C144 & pCLA101 & $\begin{array}{l}\text { Embedding ORBS while “killing" } \\
\text { the WT Shine-Dalgarno sequence }\end{array}$ & C145 \\
\hline pCLA194 & QC & C146 + C147 & pCLA193 & $\begin{array}{l}\text { sfGFP gene exchanged by } \\
\text { sequence for GCN4 16-31 }\end{array}$ & C145 \\
\hline
\end{tabular}




\subsection{Sequence Alignments}

\subsubsection{PylS Promoter Library}

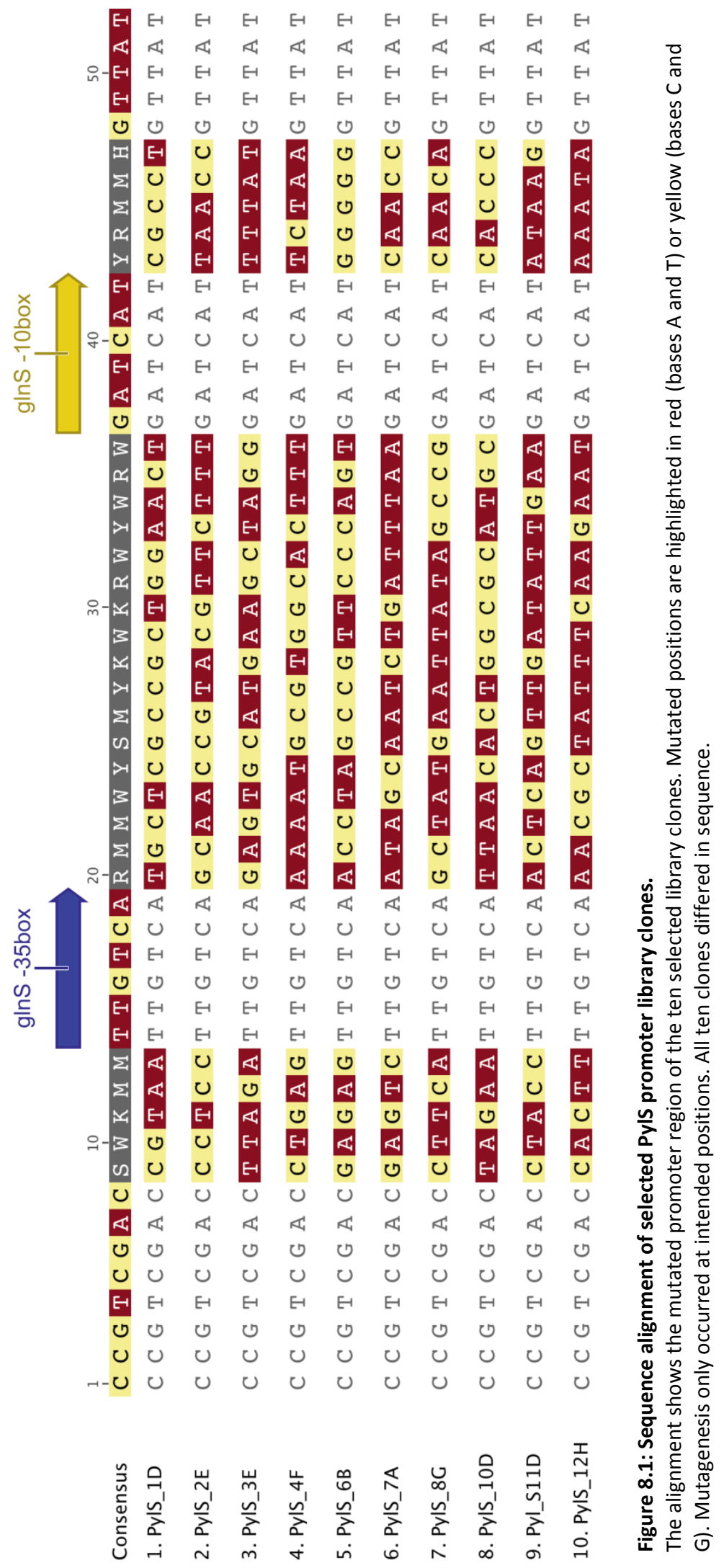




\subsubsection{MjYRS_AGGA Promoter Library}

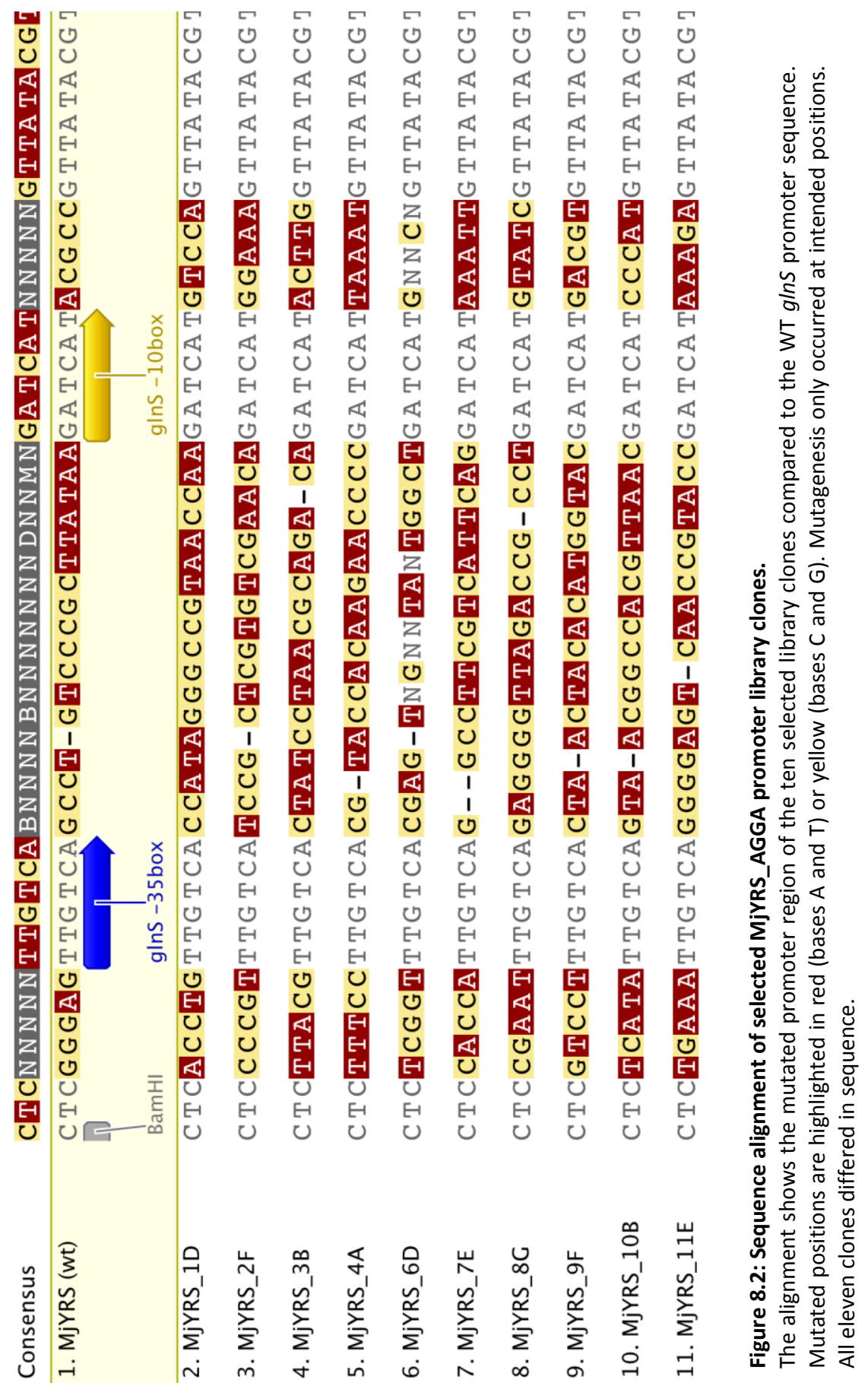




\subsubsection{PylT Promoter Library}

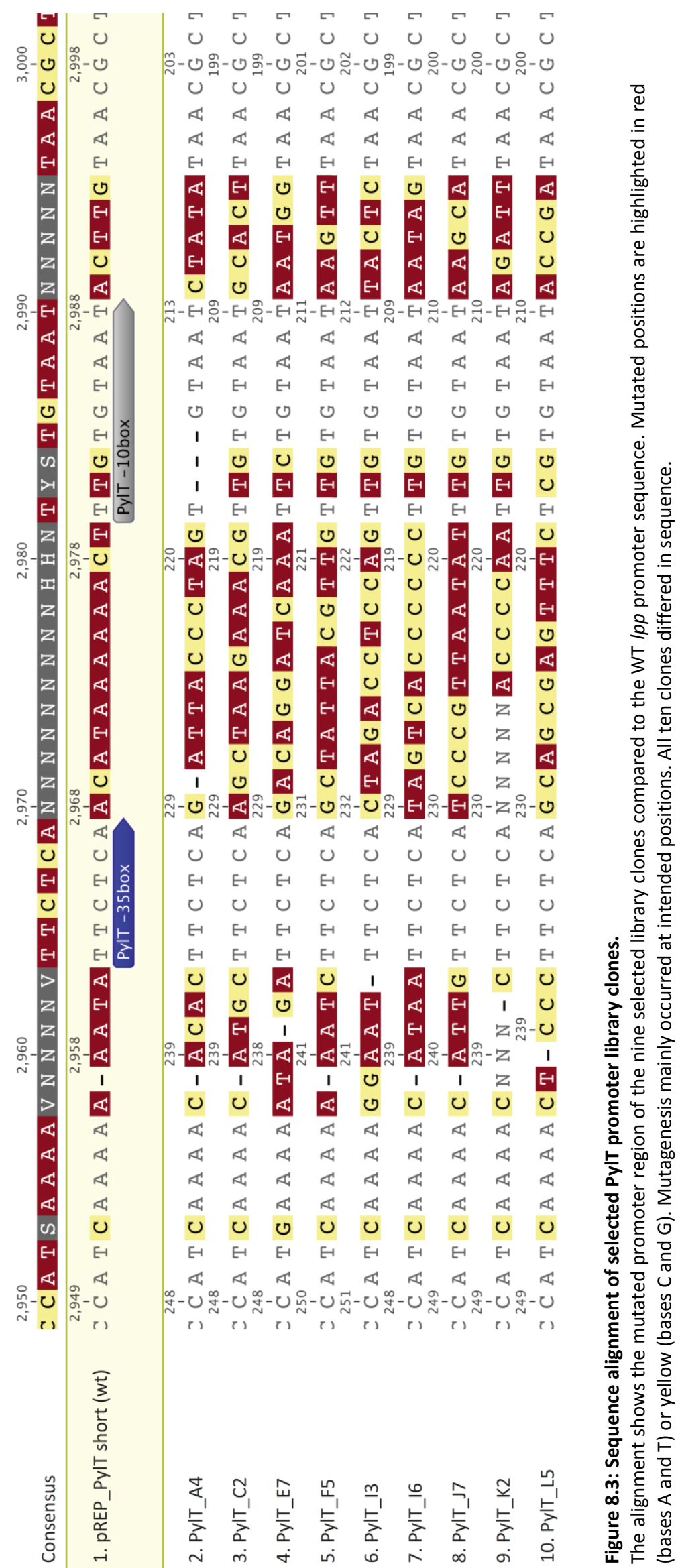




\subsubsection{MjYT_UCCU Promoter Library}

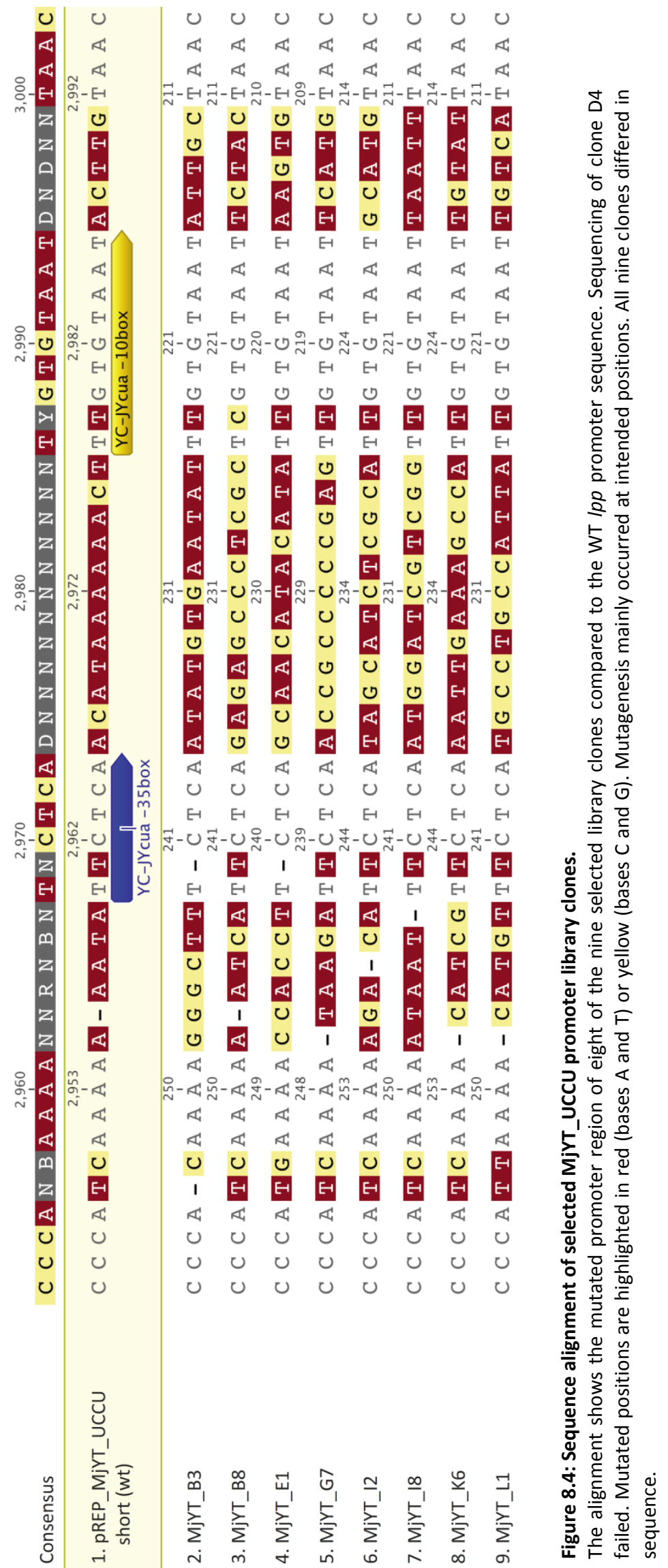




\subsection{In-Cell sfGFP Fluorescence Measurements}

\subsubsection{Initial Conditions For In-Cell NMR Measurements}

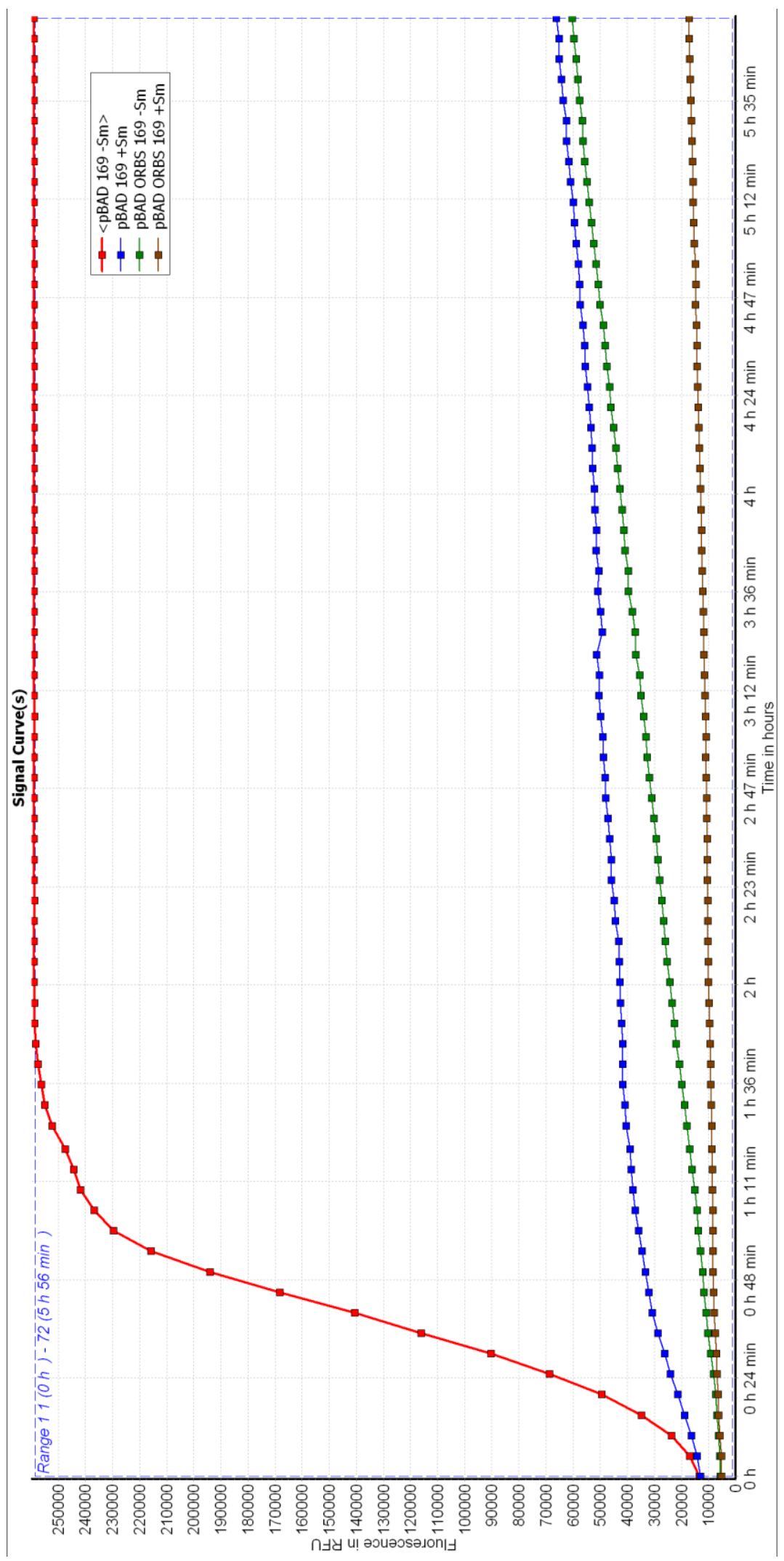

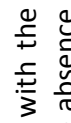

을

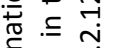

言 월

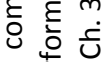

$\subseteq \frac{\mathrm{d}}{\mathrm{d}}$

区

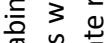

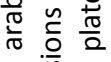

ลำ

ก.

i⿱

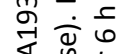

㟧

宊

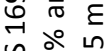

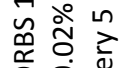

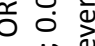

定容嵒

은

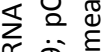

है के

즌

。응 잉

ㅇ. 능

它

¿

ษั

ญำ 돈

هั

흘 흥

实

ปั $\subseteq \frac{0}{0}$

.

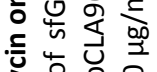

牙然

흥 음

过这立

흥 옹

항 है

这

昰.

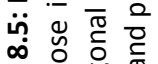

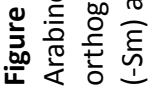




\subsubsection{Optimization For In-Cell NMR Measurements}

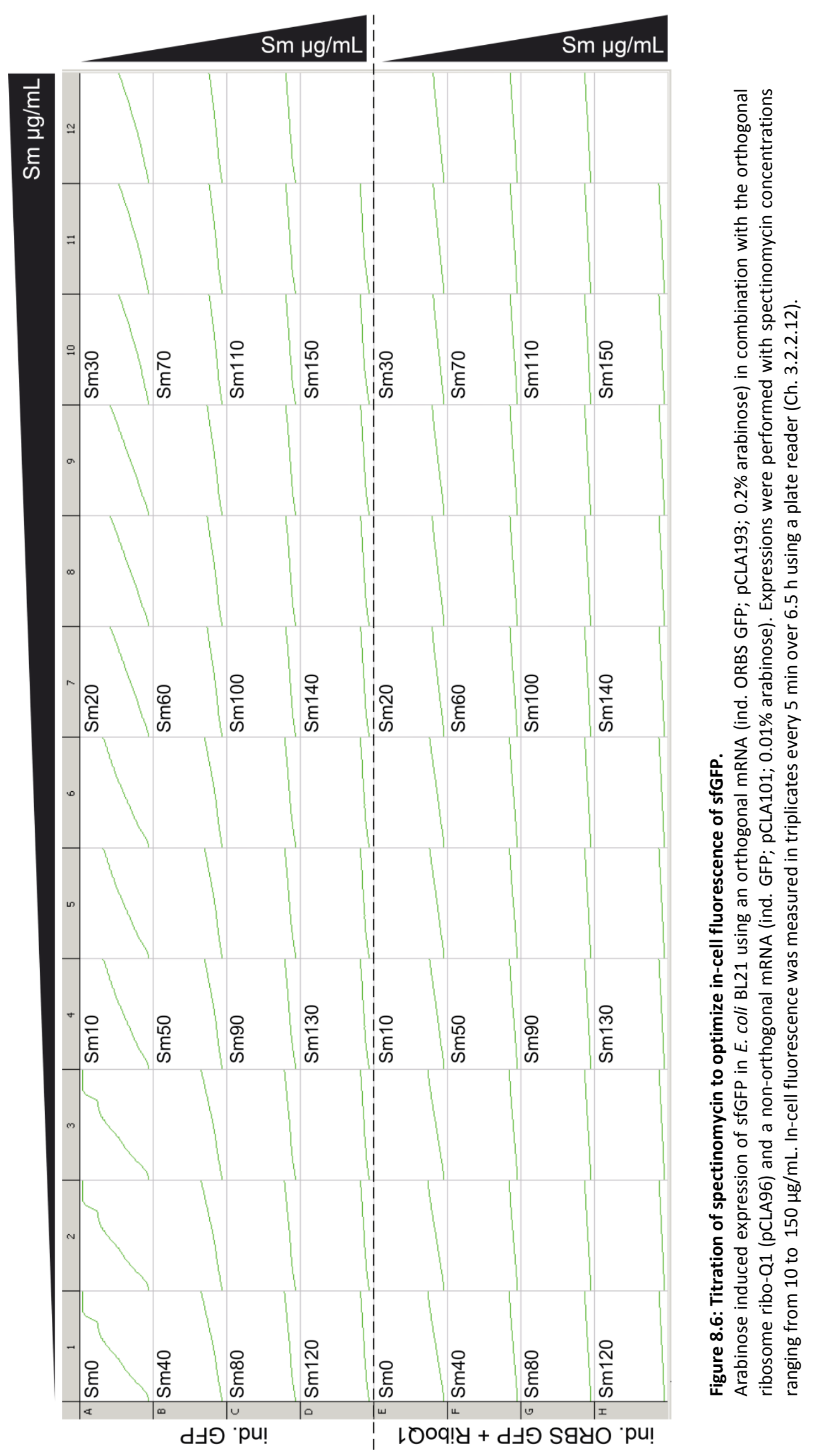




\subsection{Curriculum Vitae}

$\begin{array}{ll}\text { Name: } & \text { Christoph Lammers } \\ \text { Geburtsdatum, -ort: } & \text { 02. März } 1984 \text { in Würselen } \\ \text { Familienstand: } & \text { ledig } \\ \text { Staatsangehörigkeit: } & \text { deutsch } \\ \text { Anschrift: } & \text { Annastraße 66 / App. 65, 37075 Göttingen } \\ \text { Email: } & \text { clammer1@gwdg.de }\end{array}$

PROMOTION

Seit 2010

Promotion an der Georg-August-Universität, Göttingen

Abteilung für Angewandte Synthetische Biologie

\section{STUDIUM DER BIOLOGIE}

$09 / 2009$

Abschluss des Studiums als Diplom-Biologe

Thema der Diplomarbeit: „Erstellung eines Wikis zu den Genen von Bacillus subtilis" (Abteilung für

Allgemeine Mikrobiologie bei Prof. Dr. Stülke)

Studium der Biologie an der Georg-August-Universität, Göttingen

Hauptfach: Mikrobiologie

Nebenfächer: Biochemie, organische Chemie

\section{WEHRDIENST}

$2003-2004$

Soldat im Grundwehrdienst (Sanitäter)

4./Sanitätsregiment 12, Fürstenau

\section{SCHULISCHE AUSBILDUNG}

$1996-2003$

Ubbo-Emmius-Gymnasium, Leer

Abschluss: Allgemeine Hochschulreife

$1994-1996$

Orientierungsstufe, Collhusen

$1990-1994$

Grundschule, Ihrhove 